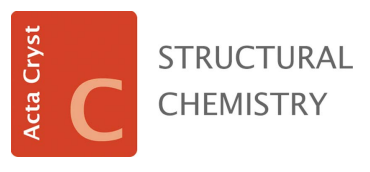

ISSN 2053-2296

Received 12 January 2019

Accepted 20 March 2019

Edited by D. R. Turner, University of Monash, Australia

Keywords: metronidazole benzoate; crystal engineering; cocrystal; salt; physicochemical; crystal structure; pharmaceutical; API; cocrystal.

CCDC references: $1890422 ; 1890421$; 1890430; 1890426; 1890432; 1890427; 1890429; 1890433; 1890424; 1890423; $1890428 ; 1890431 ; 1890425$

Supporting information: this article has supporting information at journals.iucr.org/c

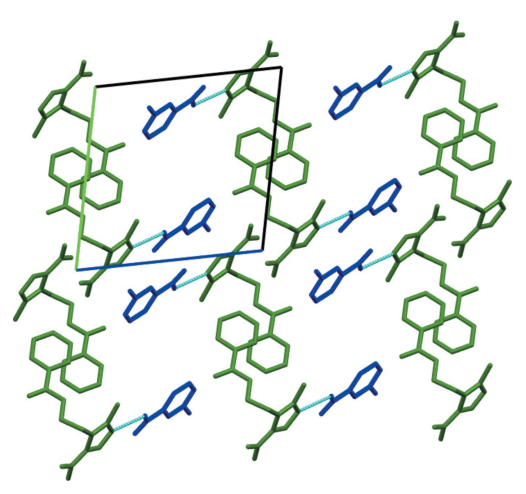

(C) 2019 International Union of Crystallography

\section{The design of novel metronidazole benzoate structures: exploring stoichiometric diversity}

\author{
Yara Santiago de Oliveira, ${ }^{a}$ Wendell Saraiva Costa, ${ }^{a}$ Poliana Ferreira Borges, ${ }^{b}$ \\ Maria Silmara Alves de Santana ${ }^{c}$ and Alejandro Pedro Ayala ${ }^{\mathrm{c} *}$
}

\begin{abstract}
${ }^{\mathbf{a}}$ Department of Pharmacy, Federal University of Ceará, Fortaleza, Ceará, Brazil, ${ }^{\mathbf{b}}$ Department of Chemical Engineering, Federal University of Ceará, Fortaleza, Ceará, Brazil, and ${ }^{\mathbf{c}}$ Department of Physics, Federal University of Ceará, Fortaleza, Ceará, Brazil. *Correspondence e-mail: ayala@fisica.ufc.br
\end{abstract}

The use of supramolecular synthons as a strategy to control crystalline structure is a crucial factor in developing new solid forms with physicochemical properties optimized by design. However, to achieve this objective, it is necessary to understand the intermolecular interactions in the context of crystal packing. The feasibility of a given synthon depends on its flexibility to combine the drug with a variety of coformers. In the present work, the imidazole-hydroxy synthon is investigated using as the target molecule benzoylmetronidazole [BZMD; systematic name 2-(2-methyl-5-nitro-1H-imidazol-1-yl)ethyl benzoate], whose imidazole group seems to be a suitable acceptor for hydrogen bonds. Thus, coformers with carboxylic acid and phenol groups were chosen. According to the availability of binding sites presented in the coformer, and considering the proposed synthon and hydrogen-bond complementarity as major factors, different drug-coformer stoichiometric ratios were explored (1:1, 2:1 and 3:1). Thirteen new solid forms (two salts and eleven cocrystals) were produced, namely BZMD-benzoic acid (1/1), $\mathrm{C}_{13} \mathrm{H}_{13} \mathrm{~N}_{3} \mathrm{O}_{4} \cdot \mathrm{C}_{7} \mathrm{H}_{6} \mathrm{O}_{2}$, BZMD- $\beta$-naphthol (1/1), $\mathrm{C}_{13} \mathrm{H}_{13} \mathrm{~N}_{3} \mathrm{O}_{4} \cdot \mathrm{C}_{10} \mathrm{H}_{8} \mathrm{O}$, BZMD-4-methoxybenzoic acid (1/1), $\mathrm{C}_{13} \mathrm{H}_{13} \mathrm{~N}_{3} \mathrm{O}_{4} \cdot \mathrm{C}_{8} \mathrm{H}_{8} \mathrm{O}_{3}$, BZMD-3,5-dinitrobenzoic acid (1/1), $\mathrm{C}_{13} \mathrm{H}_{13} \mathrm{~N}_{3} \mathrm{O}_{4} \cdot \mathrm{C}_{7} \mathrm{H}_{4} \mathrm{~N}_{2} \mathrm{O}_{6}$, BZMD-3-aminobenzoic acid (1/1), $\mathrm{C}_{13} \mathrm{H}_{13} \mathrm{~N}_{3} \mathrm{O}_{4} \cdot \mathrm{C}_{7} \mathrm{H}_{7} \mathrm{NO}_{2}, \quad$ BZMD-salicylic acid (1/1), $\mathrm{C}_{13} \mathrm{H}_{13} \mathrm{~N}_{3} \mathrm{O}_{4} \cdot \mathrm{C}_{7} \mathrm{H}_{6} \mathrm{O}_{3}, \mathrm{BZMD}-$ maleic acid (1/1) \{as the salt 1-[2-(benzoyloxy)ethyl]-2-methyl-5-nitro-1 $\mathrm{H}$-imidazol-3-ium 3-carboxyprop-2-enoate\}, $\mathrm{C}_{13} \mathrm{H}_{14}$ $\mathrm{N}_{3} \mathrm{O}_{4}{ }^{+} \cdot \mathrm{C}_{4} \mathrm{H}_{3} \mathrm{O}_{4}{ }^{-}$, BZMD-isophthalic acid (1/1), $\mathrm{C}_{13} \mathrm{H}_{13} \mathrm{~N}_{3} \mathrm{O}_{4} \cdot \mathrm{C}_{8} \mathrm{H}_{6} \mathrm{O} 4$, BZMDresorcinol (2/1), $2 \mathrm{C}_{13} \mathrm{H}_{13} \mathrm{~N}_{3} \mathrm{O}_{4} \cdot \mathrm{C}_{6} \mathrm{H}_{6} \mathrm{O}_{2}, \quad$ BZMD-fumaric acid (2/1), $\mathrm{C}_{13} \mathrm{H}_{13} \mathrm{~N}_{3} \mathrm{O}_{4} \cdot 0.5 \mathrm{C}_{4} \mathrm{H}_{4} \mathrm{O}_{4}$, BZMD-malonic acid (2/1), $2 \mathrm{C}_{13} \mathrm{H}_{13} \mathrm{~N}_{3} \mathrm{O}_{4} \cdot \mathrm{C}_{3} \mathrm{H}_{2} \mathrm{O}_{4}$, BZMD-2,6-dihydroxybenzoic acid (1/1) \{as the salt 1-[2-(benzoyloxy)ethyl]-2methyl-5-nitro- $1 \mathrm{H}$-imidazol-3-ium 2,6-dihydroxybenzoate, $\mathrm{C}_{13} \mathrm{H}_{14} \mathrm{~N}_{3} \mathrm{O}_{4}{ }^{+} \cdot \mathrm{C}_{7} \mathrm{H}_{5} \mathrm{O}_{4}{ }^{-}$, and BZMD-3,5-dihydroxybenzoic acid (3/1), $3 \mathrm{C}_{13} \mathrm{H}_{13} \mathrm{~N}_{3} \mathrm{O}_{4} \cdot \mathrm{C}_{7} \mathrm{H}_{6} \mathrm{O}_{4}$, and their crystalline structures elucidated, confirming the robustness of the selected synthon.

\section{Introduction}

The understanding of intermolecular interactions in the context of crystal packing and the ability to predict them using supramolecular synthons as a strategy to control crystal structure are key factors in the generation of new solid forms with a desired set of physicochemical properties (Corpinot \& Bučar, 2018; Desiraju, 2010). This research area has developed rapidly due to the possibility of rationally designing structures of pharmaceutical compounds (Babu et al., 2012; Blagden et al., 2007; Bolla \& Nangia, 2016), improving their physicochemical properties with tailored pharmacokinetics and mechanical properties, such as solubility, stability, flowability and compressibility (Bolla \& Nangia, 2016). Further advances have confirmed that the path for the development of a new form depends on clear steps, which are no longer based only 
on serendipity, but on the rational design of solid forms as part of a field known as crystal engineering (Kavanagh et al., 2019).

The adopted strategy combines the ability to rationally design supramolecular structures and the modulation of the undesired physicochemical properties of the drug without any covalent modification in the molecules (Berry \& Steed, 2017). According to the availability of binding sites present in the coformer, and considering the proposed synthon and hydrogen-bond complementarity as major factors (Wang et al., 2018), different drug-coformer stoichiometric ratios can be explored. Thus, the first step on the development path is to identify and select potential hydrogen-bonding sites in the active pharmaceutical ingredient (API) and to propose appropriate coformers with complementary groups (synthons) (Aitipamula et al., 2014). The initial prospection is vitally important for discovering supramolecular structures with pharmaceutical relevance (Kavanagh et al., 2019; Saha \& Desiraju, 2018).

The possibility of generating different solid forms from the API-coformer combination (Berry \& Steed, 2017; Cerreia Vioglio et al., 2017; Healy et al., 2017; Shaikh et al., 2018) has consolidated drug development based on pharmaceutical cocrystals, since there is strong scientific evidence corroborating their potential to provide viable candidates for solid formulations having 'by design' properties. Furthermore, a growing interest of the pharmaceutical industry to use these products has been observed, once they are a reality on the market. Thus, we can mention the cocrystals Suglat (ipragliflozin and L-proline), Steglatro (ertugliflozin and L-pyroglutamic acid) and Entresto (valsartan and sacubitril) as successful examples of commercialization (Kavanagh et al., 2019). The growing interest in using pharmaceutical cocrystals have led to the US Food and Drug Administration (FDA, 2018) and the European Medicines Agency (EMA, 2015) publishing position papers related to them. These documents define a regulatory framework, providing guidance to industry and expanding the records for cocrystals.

The target molecule used in this work to apply crystaldesign principles was benzoylmetronidazole (BZMD) (Fig. 1), also known as metronidazole benzoate. This drug is indicated for the treatment of infections caused by a wide range of anaerobic, protozoan and bacteroid bacteria, including trichomoniasis, amebiasis, vaginosis and gingivitis (Bempong et al., 2005; Caira et al., 1993). Hoelgaard \& Møller (1983) reported two anhydrous (anhydrates I and II) and one monohydrate (BZMDH) form of BZMD, but just the monohydrate and the commercial anhydrous forms have had their crystallographic structures elucidated (Caira et al., 1993). However, none of these structures is reported in the Cambridge Structural Database (CSD; Groom et al., 2016). BZMD anhydrate I crystallizes as blocks or elongated prisms, while the monohydrate form has hexagonally shaped prisms. Both anhydrous forms of BZMD crystallize in triclinic systems, belonging to the $P \overline{1}$ space group but differing in the

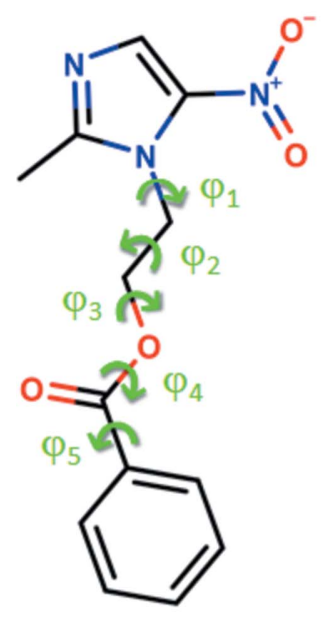

Metronidazole benzoate $(\mathrm{BZMD})$

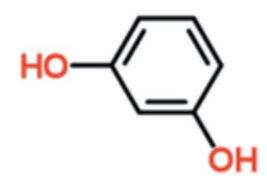

Resorcinol (RES)

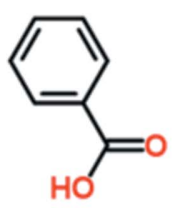

Benzoic acid (BZC)

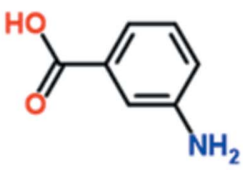

3-Aminobenzoic acid $(\mathrm{ABN})$

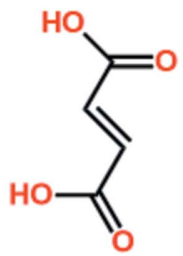

Fumaric acid

(FMA)<smiles>Oc1ccc2ccccc2c1</smiles>

B-naphthol (NAF)<smiles>O=C(O)c1ccccc1O</smiles>

Salicylic acid (SLC)

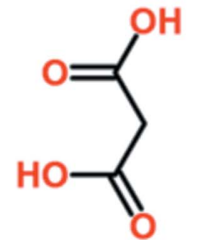

Malonic acid (MLN)

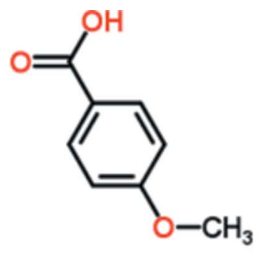
4-Methoxybenzoic acid (MAC)

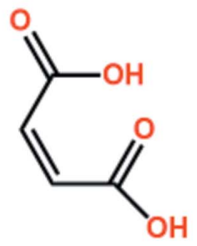

Maleic acid (MLC)

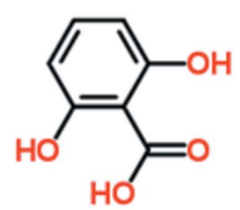

2,6Dihydroxybenzoic acid (2,6-DBA)<smiles>O=C(O)c1cc([N+](=O)[O-])cc([N+](=O)[O-])c1</smiles>

3,5-Dinitrobenzoic acid (3,5-DNZ)

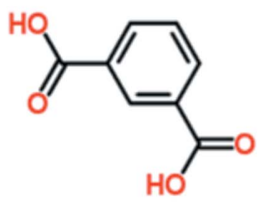

Isophthalic acid (IAC)

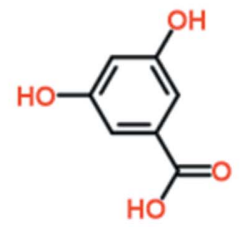

3,5-

Dihydroxybenzoic acid (3,5-DBA)

Figure 1

Metronidazole benzoate, cocrystal and salt formers with their corresponding abbreviations. $\varphi_{i}$ symbolizes the backbone dihedral angles. 
Table 1

Experimental details.

For all determinations, a multi-scan absorption correction (SADABS; Bruker, 2016) was used.

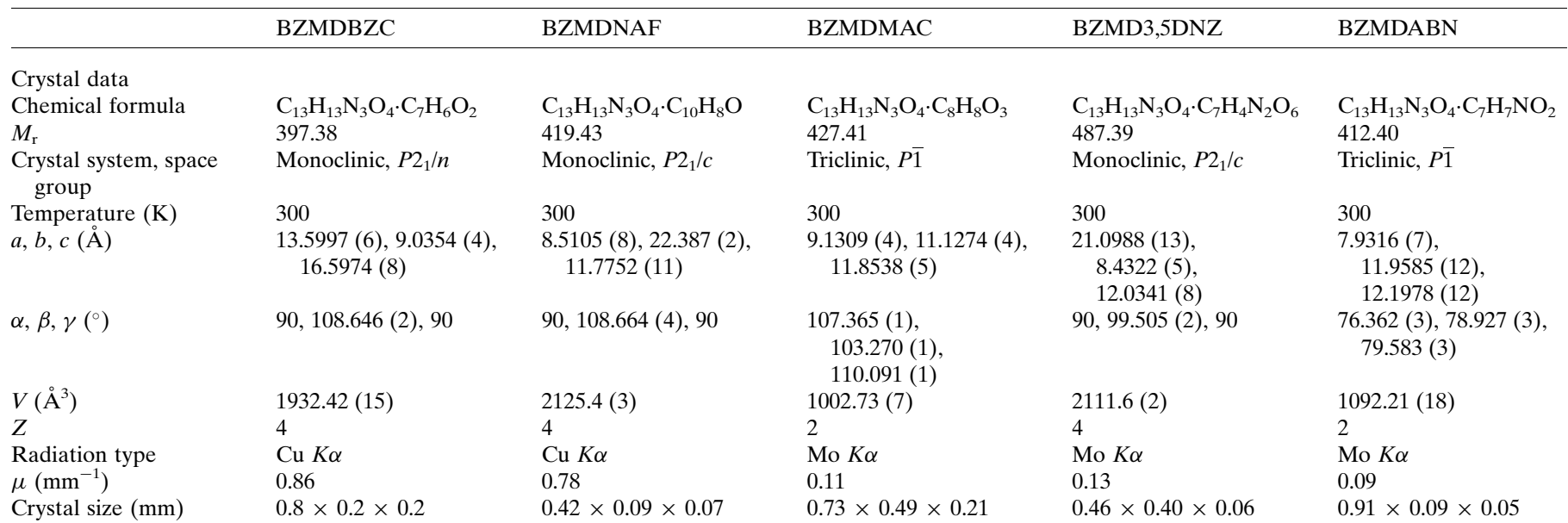

Data collection

Diffractometer

$T_{\min }, T_{\max }$

No. of measured, independent and observed $[I>2 \sigma(I)]$ reflections

$R_{\text {int }}$

$(\sin \theta / \lambda)_{\max }\left(\AA^{-1}\right)$

0.053

Bruker APEXII CCD

\section{Bruker D8 VENTURE Kappa Duo PHOTON II CPAD \\ $0.678,1.000$}

$0.675,0.753$

60527, 3542, 2669

$39131,3884,2810$

0.084

0.603

$0.058,0.193,1.06$

$R\left[F^{2}>2 \sigma\left(F^{2}\right)\right]$, $w R\left(F^{2}\right), S$

No. of reflections

No. of parameters

No. of restraints

$\mathrm{H}$-atom treatment
$0.045,0.130,1.07$

3542

339

0

All $\mathrm{H}$-atom parameters refined
3884

365

0

All H-atom parameters refined
Bruker APEX3 microsource

$0.587,0.746$

$32021,4583,3795$

0.063

0.650

$0.059,0.165,1.05$

4583

364

0

All H-atom parameters refined

$0.76,-0.24$

$0.30,-0.23$

Chemical formula

$M_{\mathrm{r}}$

Crystal system, space group

Temperature (K)

$a, b, c(\AA)$

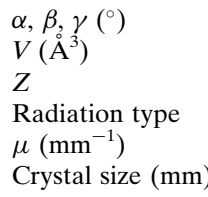

Data collection

Diffractometer

$T_{\min }, T_{\max }$

No. of measured, independent and observed $[I>2 \sigma(I)]$ reflections
BZMDMLC

\section{$\mathrm{C}_{13} \mathrm{H}_{14} \mathrm{~N}_{3} \mathrm{O}_{4}{ }^{+} \cdot \mathrm{C}_{4} \mathrm{H}_{3} \mathrm{O}_{4}{ }^{-}$ 391.33}

$\mathrm{C}_{13} \mathrm{H}_{13} \mathrm{~N}_{3} \mathrm{O}_{4} \cdot \mathrm{C}_{7} \mathrm{H}_{6} \mathrm{O}_{3}$ 413.38

Monoclinic, $P 2_{1} / \mathrm{c}$

301

$9.5055(6)$,

31.2239 (19),

6.8578 (4)

90, 102.628 (2), 90

1986.2 (2)

4

Mo $K \alpha$

0.11

$0.68 \times 0.24 \times 0.16$

Monoclinic, $C 2 / c$

302

43.130 (3), 5.7944 (4), 15.2645 (11)

90, 109.600 (3), 90 3593.7 (5)

8

Mo $K \alpha$

0.12

$0.57 \times 0.23 \times 0.05$

BZMDIAC

$\mathrm{C}_{13} \mathrm{H}_{13} \mathrm{~N}_{3} \mathrm{O}_{4} \cdot \mathrm{C}_{8} \mathrm{H}_{6} \mathrm{O}_{4}$

441.39

Monoclinic, $P 2_{1} / c$

304

8.8300 (3),

$33.7182(10)$

$7.3199(2)$

90, 107.878 (1), 90

2074.13 (11)

4

Mo $K \alpha$

0.11

$0.95 \times 0.3 \times 0.11$

Bruker D8 VENTURE

Kappa Duo

PHOTON II CPAD

$0.261,0.746$

20803, 4130, 2159

$\begin{array}{ll}\text { Bruker D8 VENTURE } & \text { Bruker APEXII CCD } \\ \text { Kappa Duo } & \\ \text { PHOTON II CPAD } & \\ 0.649,0.746 & 0.620,0.746 \\ 38842,6344,4809 & 46849,3705,2625\end{array}$

$46849,3705,2625$
$\mathrm{C}_{13} \mathrm{H}_{13} \mathrm{~N}_{3} \mathrm{O}_{4} \cdot 0.5 \mathrm{C}_{4} \mathrm{H}_{4} \mathrm{O}_{4}$ 333.30

Monoclinic, $P 2_{1} / \mathrm{c}$

300

$9.0358(5)$,

26.6419 (14), 6.8796 (3)

90, 102.419 (2), 90

1617.38 (14)

4

Mo $K \alpha$

0.11

$0.63 \times 0.17 \times 0.17$
Bruker APEXII CCD

$0.587,0.746$

$38219,5008,2711$

0.090

0.649

$0.054,0.168,1.01$

5008

340

0

$\mathrm{H}$ atoms treated by a mixture of independent and constrained refinement $0.25,-0.19$

BZMD2,6DBA

$\mathrm{C}_{13} \mathrm{H}_{14} \mathrm{~N}_{3} \mathrm{O}_{4}{ }^{+} \cdot \mathrm{C}_{7} \mathrm{H}_{5} \mathrm{O}_{4}{ }^{-}$ 429.38

Monoclinic, $P 2_{1} / n$

301

8.2443 (4), 15.9009 (7), $15.4526(8)$

90, 102.454 (2), 90 1978.04 (17)

4

Mo $K \alpha$

0.11

$0.83 \times 0.67 \times 0.27$

Bruker D8 VENTURE
Kappa Duo
PHOTON II CPAD
$0.490,0.746$
$27674,4348,3408$


Table 1 (continued)

\begin{tabular}{|c|c|c|c|c|c|}
\hline & BZMDSLC & BZMDMLC & BZMDIAC & BZMDFMA & BZMD2,6DBA \\
\hline$R_{\text {int }}$ & 0.035 & 0.089 & 0.037 & 0.066 & 0.076 \\
\hline$(\sin \theta / \lambda)_{\max }\left(\AA^{-1}\right)$ & 0.650 & 0.650 & 0.715 & 0.650 & 0.651 \\
\hline \multicolumn{6}{|l|}{ Refinement } \\
\hline $\begin{array}{l}R\left[F^{2}>2 \sigma\left(F^{2}\right)\right] \\
\quad w R\left(F^{2}\right), S\end{array}$ & $0.042,0.115,1.06$ & $0.056,0.147,1.02$ & $0.050,0.145,1.04$ & $0.051,0.151,1.05$ & $0.069,0.185,1.10$ \\
\hline No. of reflections & 4553 & 4130 & 6344 & 3705 & 4348 \\
\hline No. of parameters & 347 & 322 & 365 & 267 & 356 \\
\hline No. of restraints & 100 & 0 & 0 & 0 & 0 \\
\hline H-atom treatment & $\begin{array}{l}\text { All } \mathrm{H} \text {-atom parameters } \\
\text { refined }\end{array}$ & $\begin{array}{l}\text { All H-atom parameters } \\
\text { refined }\end{array}$ & $\begin{array}{l}\text { All H-atom parameters } \\
\text { refined }\end{array}$ & $\begin{array}{l}\text { H atoms treated by a } \\
\text { mixture of indepen- } \\
\text { dent and constrained } \\
\text { refinement }\end{array}$ & $\begin{array}{l}\text { All H-atom parameters } \\
\text { refined }\end{array}$ \\
\hline$\Delta \rho_{\max }, \Delta \rho_{\min }\left(\mathrm{e} \AA^{-3}\right)$ & $0.17,-0.20$ & $0.18,-0.22$ & $0.20,-0.21$ & $0.39,-0.33$ & $0.37,-0.27$ \\
\hline
\end{tabular}

\begin{tabular}{|c|c|c|c|}
\hline & BZMD3,5DBA & BZMDRES & BZMDMLN \\
\hline \multicolumn{4}{|l|}{ Crystal data } \\
\hline Chemical formula & $3 \mathrm{C}_{13} \mathrm{H}_{13} \mathrm{~N}_{3} \mathrm{O}_{4} \cdot \mathrm{C}_{7} \mathrm{H}_{6} \mathrm{O}_{4}$ & $2 \mathrm{C}_{13} \mathrm{H}_{13} \mathrm{~N}_{3} \mathrm{O}_{4} \cdot \mathrm{C}_{6} \mathrm{H}_{6} \mathrm{O}_{2}$ & $2 \mathrm{C}_{13} \mathrm{H}_{13} \mathrm{~N}_{3} \mathrm{O}_{4} \cdot \mathrm{C}_{3} \mathrm{H}_{2} \mathrm{O}_{4}$ \\
\hline$M_{\mathrm{r}}$ & 979.91 & 660.63 & 652.57 \\
\hline Crystal system, space group & Monoclinic, $P 2_{1} / n$ & Orthorhombic, $\mathrm{Pbca}$ & Orthorhombic, $P b c n$ \\
\hline Temperature $(\mathrm{K})$ & 300 & 300 & 300 \\
\hline$a, b, c(\AA)$ & $7.1481(2), 36.7892(8), 18.0584(4)$ & $26.3241(4), 7.1612(1), 33.8433(5)$ & $26.1542(4), 7.2708(1), 16.6719$ (3) \\
\hline$\alpha, \beta, \gamma\left(^{\circ}\right)$ & $90,100.451(1), 90$ & $90,90,90$ & $90,90,90$ \\
\hline$V\left(\AA^{3}\right)$ & $4670.1(2)$ & $6379.87(16)$ & $3170.36(9)$ \\
\hline$Z$ & 4 & 8 & 4 \\
\hline Radiation type & $\mathrm{Cu} K \alpha$ & $\mathrm{Cu} K \alpha$ & $\mathrm{Cu} K \alpha$ \\
\hline$\mu\left(\mathrm{mm}^{-1}\right)$ & 0.91 & 0.88 & 0.92 \\
\hline Crystal size $(\mathrm{mm})$ & $0.28 \times 0.14 \times 0.06$ & $0.25 \times 0.12 \times 0.11$ & $0.46 \times 0.29 \times 0.13$ \\
\hline \multicolumn{4}{|l|}{ Data collection } \\
\hline Diffractometer & Bruker APEXII CCD & Bruker APEXII CCD & Bruker APEXII CCD \\
\hline$T_{\min }, T_{\max }$ & $0.835,0.986$ & $0.623,0.753$ & $0.736,0.907$ \\
\hline $\begin{array}{l}\text { No. of measured, independent and } \\
\text { observed }[I>2 \sigma(I)] \text { reflections }\end{array}$ & $56516,8547,5499$ & $121051,5865,4816$ & $54755,2893,2423$ \\
\hline$R_{\text {int }}$ & 0.058 & 0.066 & 0.038 \\
\hline$(\sin \theta / \lambda)_{\max }\left(\AA^{-1}\right)$ & 0.604 & 0.603 & 0.602 \\
\hline \multicolumn{4}{|l|}{ Refinement } \\
\hline$R\left[F^{2}>2 \sigma\left(F^{2}\right)\right], w R\left(F^{2}\right), S$ & $0.052,0.176,1.01$ & $0.048,0.147,1.03$ & $0.078,0.268,1.13$ \\
\hline No. of reflections & 8547 & 5865 & 2893 \\
\hline No. of parameters & 844 & 540 & 244 \\
\hline No. of restraints & 1 & 0 & 36 \\
\hline $\mathrm{H}$-atom treatment & $\begin{array}{l}\mathrm{H} \text { atoms treated by a mixture of } \\
\text { independent and constrained } \\
\text { refinement }\end{array}$ & $\begin{array}{l}\mathrm{H} \text { atoms treated by a mixture of } \\
\text { independent and constrained } \\
\text { refinement }\end{array}$ & $\mathrm{H}$-atom parameters constrained \\
\hline$\Delta \rho_{\max }, \Delta \rho_{\min }\left(\mathrm{e} \AA^{-3}\right)$ & $0.27,-0.22$ & $0.30,-0.31$ & $0.33,-0.27$ \\
\hline
\end{tabular}

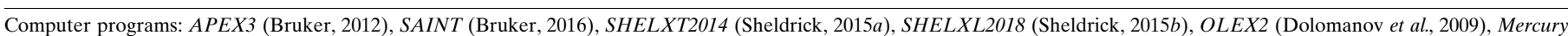
(Version 3.10; Macrae et al., 2008) and VEGA (Pedretti et al., 2004).

crystal packing and BZMD conformation. The crystalline structure of anhydrate I is mainly determined by $\mathrm{C}-\mathrm{H} \cdots \pi$ interactions and weak hydrogen bonds; among them it is relevant to emphasize the role of the $\mathrm{N}$ atom of the imidazole group as an acceptor. This fact is evident in the monohydrated form, as the simplest multicomponent BZMD structure, where water molecules act as bridges through $\mathrm{OW}-\mathrm{H} \cdots \mathrm{N}$ and OW-H. .O hydrogen bonds (Caira et al., 1993). Based on these structures, the imidazole group seems to be a suitable acceptor for hydrogen bonds with coformers containing hydroxy groups. Thus, we decided to explore this synthon by choosing coformers with carboxylic acid and phenol groups.

Additional factors, such as solvent, temperature and methodology, also influence the final product (Aitipamula et al., 2014; Sarraguça et al., 2016). Several methods can be used in order to obtain new solid forms (Delori et al., 2012; Do \&
Friscic, 2017; Douroumis et al., 2017; Hasa \& Jones, 2017; James et al., 2012). In this study, the rational design provided by crystal engineering was aligned to the traditional solventevaporation methodology (Barikah, 2018), which has been applied successfully with a large number of APIs (Diniz et al., 2018; Sarkar \& Rohani, 2015; Shayanfar \& Jouyban, 2014; Shayanfar et al., 2014). Following this strategy, thirteen new solid forms (two salts and eleven cocrystals) were obtained successfully employing crystal engineering principles by selecting suitable coformers (Fig. 1).

\section{Experimental}

\subsection{Materials}

Metronidazole benzoate was used as received from Brazilian Pharmacopeia (Brazil), whereas the coformers were 
purchased from Sigma-Aldrich [2,6-dihydroxybenzoic acid (2,6DBA), 3,5-dihydroxybenzoic acid (3,5DBA), 3,5-dinitrobenzoic acid (3,5DNZ), isophthalic acid (IAC), 4-methoxybenzoic acid (MAC) and resorcinol (RES)], Vetec [benzoic acid (BZC), salicylic acid (SLC), malonic acid (MLN) and 3-aminobenzoic acid (ABN)], Dinâmica [fumaric acid (FMA) and maleic acid (MLC)] and Merck ( $\beta$-naphthol and NAF). The solvent was purchased from Vetec and used without further purification.

\subsection{Solution growth of single crystals}

Slow evaporation (Barikah, 2018) was used to grow single crystals, using about $15 \mathrm{mg}$ of metronidazole benzoate, the corresponding amount of the selected coformer and $2 \mathrm{ml}$ of ethanol as solvent. The API-coformer stoichiometric ratio used was based on the number of $\mathrm{OH}$ groups present in each coformer. Therefore, the API-coformer ratio for BZC, NAF, MAC, 3,5DNZ and ABN was 1:1, for SLC, MLC, IAC, RES, FMA and MLN was 2:1, and for 2,6DBA and 3,5DBA was 3:1. The vials were left stirring on a hot plate. The solvent was then allowed to evaporate at room temperature. Crystals were harvested after one week.

Seven letter codes were given for the salts and cocrystals obtained, and they are used throughout this article. The first four letters are related to the drug benzoylmetronidazole [BZMD; systematic name 2-(2-methyl-5-nitro- $1 H$-imidazol-1yl)ethyl benzoate] and the last three letters identify the coformer. The title compounds are thus BZMD-benzoic acid (BZMDBZC), BZMD- $\beta$-naphthol (BZMDNAF), BZMD4-methoxybenzoic acid (BZMDMAC), BZMD-3,5-dinitrobenzoic acid (BZMD3,5DNZ), BZMD-3-aminobenzoic acid (BZMDABN), BZMD-salicylic acid (BZMDSLC), BZMDmaleic acid (BZMDMLC), BZMD-isophthalic acid (BZMDIAC), BZMD-resorcinol (BZMDRES), BZMD-fumaric acid (BZMDFMA), BZMD-malonic acid (BZMDMLN), BZMD2,6-dihydroxybenzoic acid (BZMD2,6DBA) and BZMD-3,5dihydroxybenzoic acid (BZMD3,5DBA). BZMDBZC, BZMDSLC, BZMDMLC, BZMDIAC, BZMDFMA, BZMDMLN and BZMD3,5DBA crystallized as colourless prisms, whereas BZMDMAC, BZMD3,5DNZ and BZMDABN crystallized as colourless plates. Yellow prism-shaped crystals were obtained for BZMDNAF, BZMDRES and BZMD2,6DBA. Other coformers containing carboxylic acid and phenol functional groups were tested, but only those whose structure was determined are reported in this work. A list of those coformers and the conditions used in the unsuccessful crystallization attempts is given in the supporting information.

\subsection{Single-crystal X-ray diffraction}

Crystal data, data collection and structure refinement details are summarized in Table 1.

In general, $\mathrm{H}$ atoms were located in difference Fourier maps and refined isotropically. In some methyl groups and disordered groups, the corresponding $\mathrm{H}$-atom positions were calculated geometrically and refined using the riding model. For BZMDABN, residual electron densities were observed in solvent-accessible voids associated with disordered solvent molecules and were treated with the PLATON/SQUEEZE program (Spek, 2009, 2015).

\section{Results}

\subsection{Structures}

The Cambridge Structural Database (CSD, Version 5.40 of 2018; Groom et al., 2016) contains over 900000 entries for small-molecule organic and metal-organic crystal structures. In a general search performed on this database, the synthon Imidazole-HOC produces 1554 hits, whereas Imidazolecarboxylic and Imidazole-phenol were found in 714 and 187 structures, respectively. Thus, the prevalence of synthons relating the imidazole group to carboxylic and phenol moieties are, respectively, 50 and $10 \%$, confirming that they can be used as markers in coformer selection. Based on that, this work follows the pre-established sequence depicted in the Introduction, where the first step was the selection of suitable coformers having complementary hydrogen bonds with the API through the proposed imidazole-hydroxy synthon (Im$\mathrm{HO}$ ).

3.1.1. Single donor coformers. According to the statistical analysis of the compatible molecular functional groups related to the chosen bonding site of the API, the synthon Im-HO was considered suitable. Thus, in order to verify the previous proposal, coformers with a single hydroxy donor were selected. Multicomponent structures with a 1:1 stoichiometry were produced successfully with BZC, NAF, MAC, 3,5DNZ and $A B N$. The corresponding crystalline structures are presented in Figs. 2 and 3, which can be classified as cocrystals considering the usual definition for this supramolecular organization (Aitipamula et al., 2014; Dalpiaz et al., 2017). These results demonstrate the practical feasibility of the proposed synthon, but it is also interesting to discuss the main features of the crystal packing of each cocrystal. All the interactions that help to stabilize the novel structures are listed in Table S2 of the supporting information.

It is interesting to consider, firstly, a simple carboxylic acid (BZC) and phenol (NAF), with one hydroxy group each. The crystal packing presented in Fig. 2 shows that the stronger intermolecular interaction in these structures is the imidazolehydroxy synthon. The shape of the assembled molecules gives us a better understanding of the long-range order (Corpinot \& Bučar, 2018). In the case of BZMDBZC (Fig. 2a), the $\mathrm{Im} \cdot \mathrm{H}-\mathrm{O}$ interaction is the stronger one $[1.67(3) \AA]$, but a weaker interaction involving the BZMD methyl group and the carbonyl group from the carboxylic acid moiety [2.51 (4) $\AA$ ] defines an $R_{2}^{2}(8)$ ring structure (Fig. S2 in the supporting information). BZMD and BZC form intercalated columns around the screw axis laterally linked by the previously described synthon (Fig. $2 a$ ). The $\mathrm{Im} \cdots \mathrm{H}-\mathrm{O}$ contact is also the strongest interaction [1.90 (4) $\mathrm{A}$ ], linking BZMD to the NAF coformer. In BZMDNAF (Fig. $2 b$ ), the planes of the BZMD dimers, defined by a $\mathrm{C} 6-\mathrm{H} 2 B \cdots \mathrm{O} 2$ homosynthon [2.61 (4) $\AA$, are intercalated with the planes of NAF molecules (Fig. $2 b$ ). 
Cocrystals with a 1:1 stoichiometry were also obtained with the carboxylic acids MAC (Fig. 2c) and 3,5DNZ (Fig. 2d). In the first, the API and coformer form the same $R_{2}^{2}(8)$ synthon observed in BZMDBZC (Fig. S3 in the supporting information), with similar donor-acceptor distances to those in

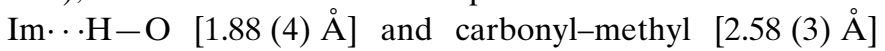
interactions. Regarding the crystal packing, BZMD-MAC dimers lie in (120) planes (Fig. $2 c$ ), which are stacked by $\pi-\pi$ interactions between the imidazole and phenyl rings from MAC [3.6911 (13) Å] and BZMD [4.0761 (15) Å]. In BZMD$3,5 \mathrm{DNZ}$, the $3,5 \mathrm{DNZ}$ molecule, linked by the target synthon [1.65 (4) $\AA$ ], is turned $62^{\circ}$ from the plane defined by the imidazole group. Furthermore, the API and coformer are also organized in layers (Fig. 2d) along the $a$ axis, where the 3,5DNZ molecules are flipped consecutively up and down along the $c$ axis as a consequence of the glide plane.

Fig. 3 shows the crystalline structure of BZMDABN, which is also defined as a carboxylic acid coformer. Different from the packing observed with other coformers and the same donor moiety, the $\mathrm{Im} \cdots \mathrm{H}-\mathrm{O}$ interaction can be identified [1.72 (4) ̊] , but the synthon is not observed. This is not the only interesting feature in BZMDABN, since large channels running along the $a$ axis are formed. This cavity is surrounded by four molecules (two APIs and two coformers), and a solvent/guest molecule could be present, characterizing an inclusion compound (Peraka et al., 2017). Some residual charges were identified during the structure resolution, which could be associated with nonstoichiometric disordered residual solvent. However, the low concentration of the solvent hindered the determination of a reliable model. The SQUEEZE method (Spek, 2015) implemented in PLATON (Spek, 2009) was applied to treat the residual charges, confirming the existence of a solvent-accessible volume of $135 \AA^{3}$ and 20 electrons, suggesting the presence of disordered ethanol, as expected considering the crystal-growing method.

3.1.2. Dual donor coformers. The previous results proved the success of the employed strategy based on the Im-HO synthon. Using the same rational design, we proposed to combine the API with coformers containing two binding sites in order to explore the production of solid forms with a 2:1 stoichiometry. In this sense, carboxylic acids and phenols with two hydroxy groups were selected. Figs. 4 and 5 show the crystal packing of the new structures.

Fig. 4 shows the crystal packing of the multicomponent forms obtained using SLC, IAC and MLC. BZMDSLC and BZMDIAC are cocrystals, whereas BZMDMLC is a salt, as
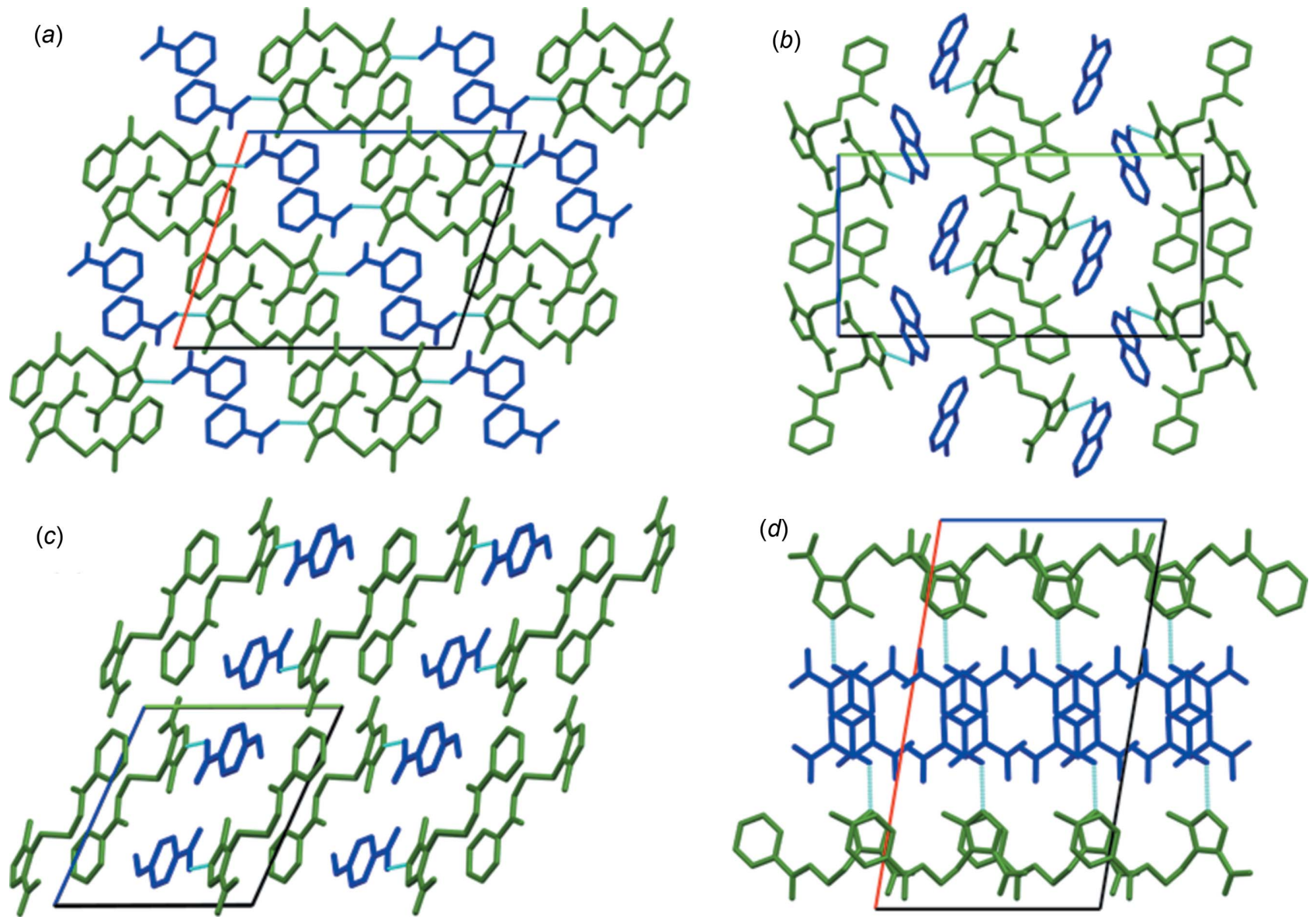

Figure 2

Projection of the crystal packing of ( $a$ ) BZMDBZC ( $b$ axis), $(b)$ BZMDNAF ( $a$ axis), $(c)$ BZMDMAC ( $a$ axis) and $(d)$ BZMD3,5DNZ ( $b$ axis). H atoms have been omitted for clarity. BZMD is represented in green and coformers in blue. Lattice axes $a, b$ and $c$ are colour-coded red, green and blue, respectively. 


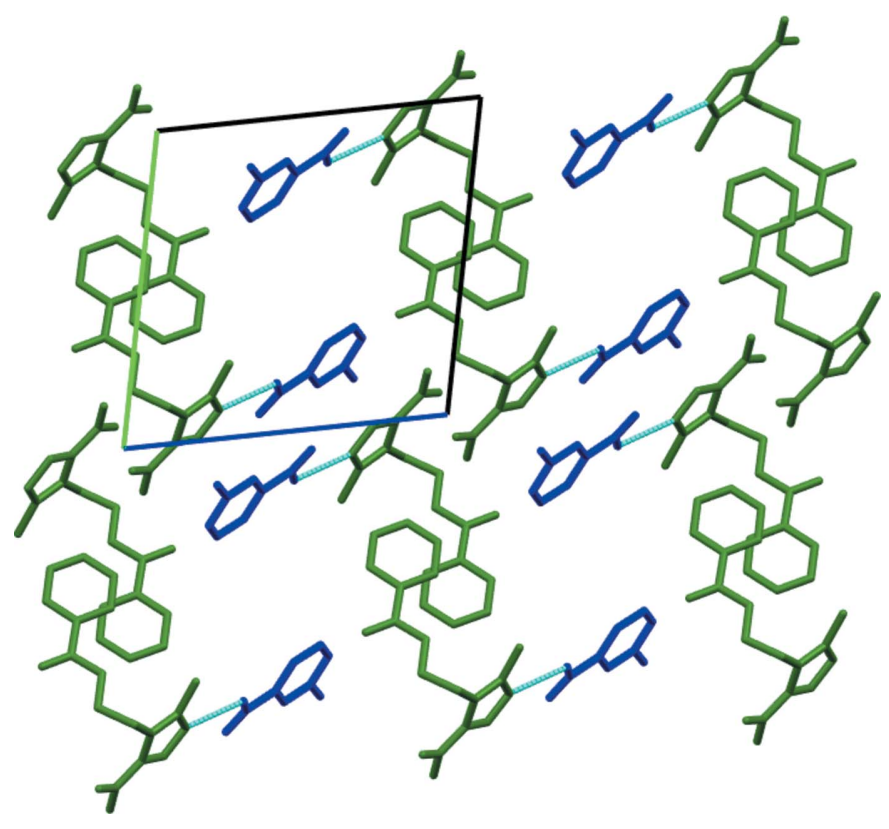

Figure 3

Projection of the crystal packing of BZMDABN ( $a$ axis), showing the solvent-accessible voids. $\mathrm{H}$ atoms have been omitted for clarity. BZMD is represented in green and coformers in blue. Lattice axes $a, b$ and $c$ are colour-coded red, green and blue, respectively.

may be verified in the ellipsoid plot included in the supporting information. Despite the fact that these coformers have two hydroxy groups, they all crystallize with a 1:1 stoichiometry. In order to understand the reasons that lead to the failure of the crystal-design strategy, it is important to discuss in detail the crystal packing of these forms.

The projection of the BZMDSLC structure along the $a$ axis shows the API and coformer intercalated columns resembling previously discussed cocrystals (Fig. 4a). The carboxy hydroxy group is involved in the $\mathrm{Im} \cdots \mathrm{H}-\mathrm{O}$ interaction [1.76 (2) $\AA$ ], but the second $\mathrm{OH}$ group of SLC does not exhibit the expected supramolecular synthon because it forms an intramolecular hydrogen bond with the carbonyl group. A similar result was obtained for the BZMDMLC salt (Fig. $4 b$ ). Even considering the charge transfer from one of the carboxylic acid groups of MLC, the same binding site is the stronger intermolecular interaction, forming an $\mathrm{Im}^{+} \ldots \mathrm{O}$ contact [1.65 (3) $\AA]$. As in BZMDSLC, the second carboxylic acid group, which is still protonated, is linked to the first carbonyl group by an intramolecular interaction thus hindering the formation of the target hydrogen bond. A common factor in SLC and MLC is that both carbonyl groups are close enough to form intramolecular hydrogen bonds, which is more favourable than the Im-HO synthon.

The IAC coformer seems to be a good candidate to avoid the previous problem because the hydroxy groups cannot form an intramolecular contact. However, a 1:1 cocrystal was obtained (Fig. 4c), where one of the hydroxy groups forms the $\mathrm{Im} \cdots \mathrm{H}-\mathrm{O}$ interaction [1.79 (3) $\mathrm{\AA}$ ], but the second $\mathrm{OH}$ group is involved in a hydrogen bond with another IAC molecule $[\mathrm{O}-\mathrm{H} \cdots \mathrm{O}=1.63(3) \AA]$. The strongly linked IAC dimers are bonded to BZMD, forming intercalated antiparallel ribbons and defining the long-range ordering of the BZMDIAC cocrystal. IAC molecules are linked by a carboxylic acid homosynthon forming an $R_{2}^{2}(8)$ ring, which is quite prevalent in carboxylic acids. Thus, it is not surprising to observe this kind of synthon in carboxylic acids, but different from the highly probable intramolecular interactions in SLC and MLC, it is not possible to predict them. Thus, from the point of view of crystal design, in order to obtain higher stoichiometric ratios, the main strategy should be to avoid coformers prone to having the selected binding sites involved in intramolecular interactions.

Based on previous results, a phenol (RES) and two carboxylic acids (FMA and MLN) were tested. Fig. 5 shows the crystal packing observed in these new solid forms, which are cocrystals with a 2:1 stoichiometry. The RES coformer fulfills the requirements of our crystal-design strategy, that is, multiple separated hydroxy groups not forming intramolecular hydrogen bonds. Thus, the two donor sites are linked to non-equivalent BZMD molecules by $\mathrm{Im} \cdots \mathrm{H}-\mathrm{O}$ interactions $[1.78(4)$ and 1.81 (4) $\AA$ ], giving rise to the

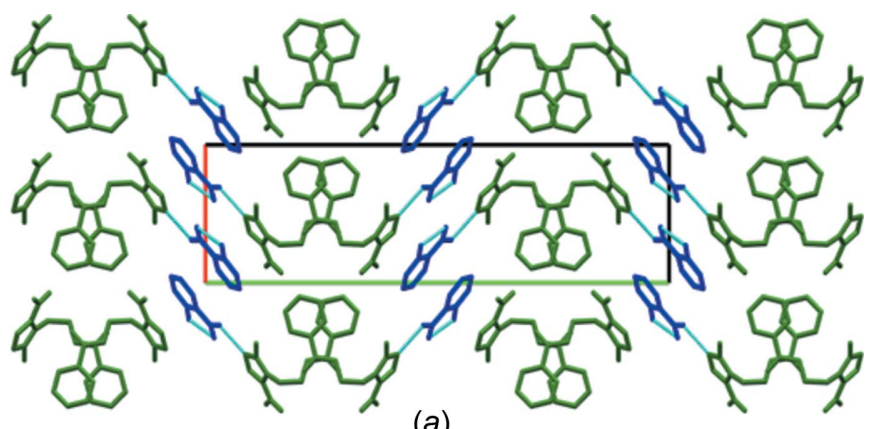

(a)

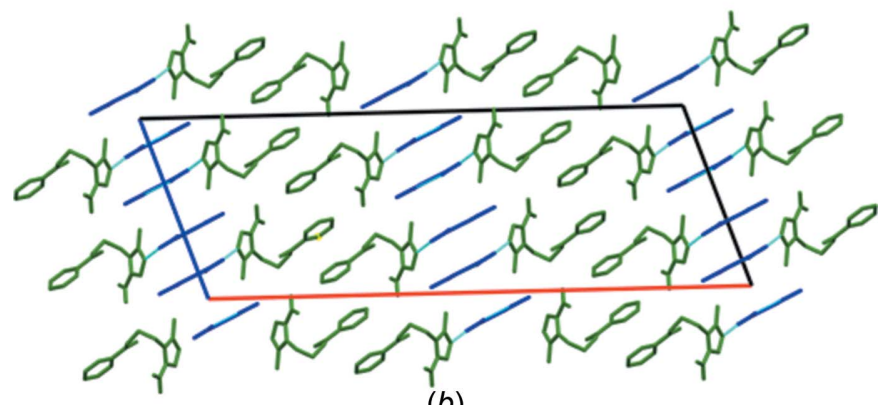

(b)

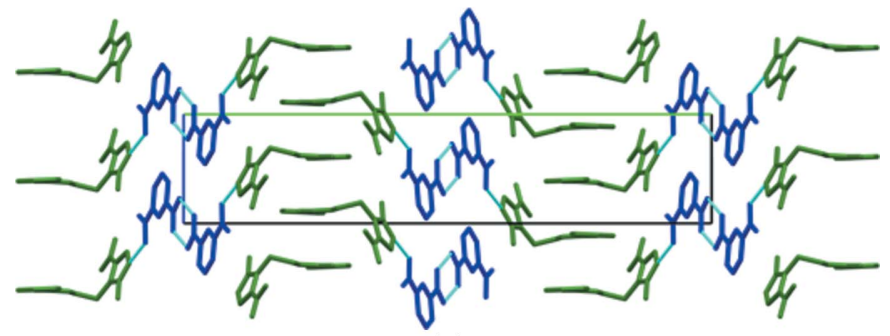

(c)

Figure 4

Projection of the crystal packing of (a) BZMDSLC ( $c$ axis), (b) BZMDMLC ( $b$ axis) and $(c)$ BZMDIAC ( $c$ axis). $\mathrm{H}$ atoms have been omitted for clarity. BZMD is represented in green and coformers in blue. Lattice axes $a, b$ and $c$ are colour-coded red, green and blue, respectively. 


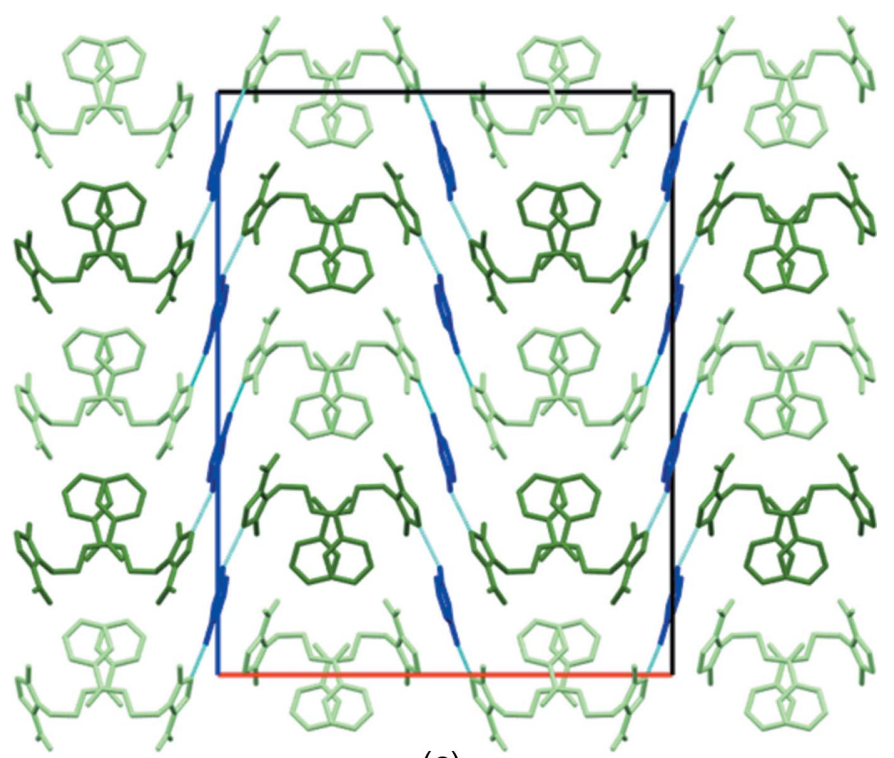

(a)

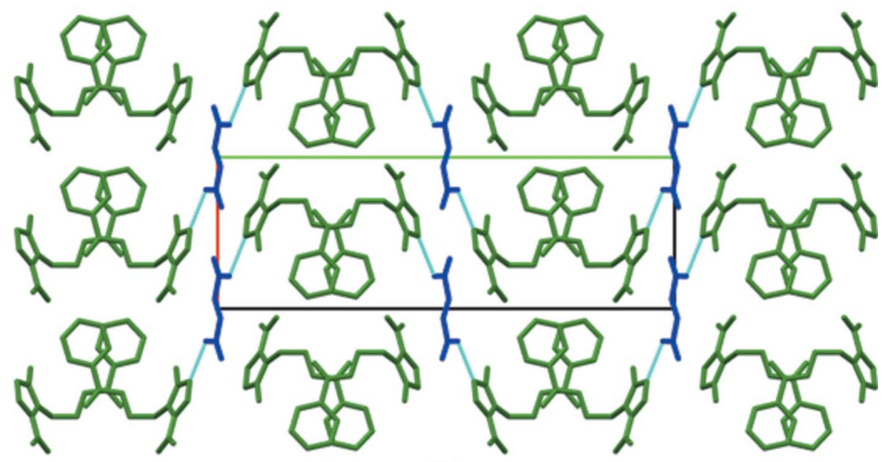

(b)

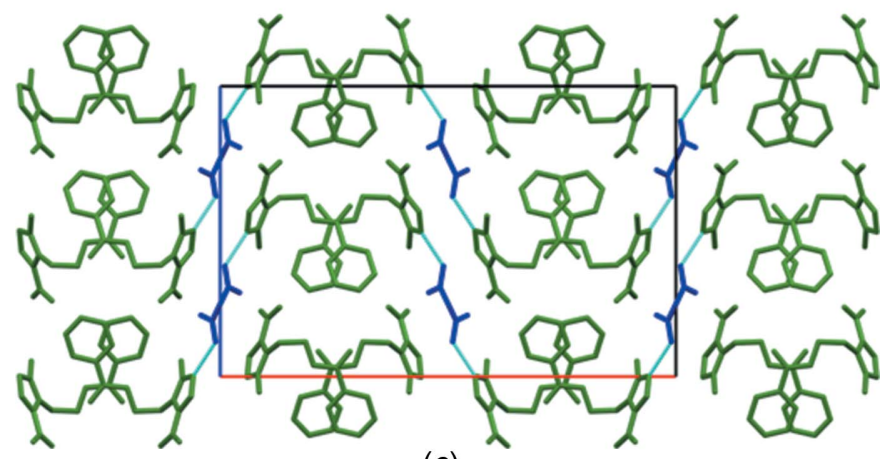

(c)

Figure 5

Projection of the crystal packing of (a) BZMDRES ( $b$ axis), $(b)$ BZMDFMA ( $c$ axis) and (c) BZMDMLN ( $c$ axis). $\mathrm{H}$ atoms have been omitted for clarity. BZMD is represented in green and coformers in blue. Lattice axes $a, b$ and $c$ are colour-coded red, green and blue, respectively.

packing of intercalated columns observed in several of the reported structures (Fig. 5a).

After observing the hindrance of the second hydroxy group in MLC due to an intramolecular interaction, a natural coformer candidate is the trans isomer FMA. A BZMDFMA cocrystal was obtained with $Z^{\prime}=0.5$. This is because the FMA molecule lies on an inversion centre and half of it is in the asymmetric unit. As a consequence, the cocrystal stoichio- metry is $2: 1$, as expected from our crystal-design strategy. Thus, the FMA coformer links two equivalent BZMD molecules by the proposed synthon [1.66 (4) $\AA$ ] , with the same crystal packing as observed in BZMDRES (Fig. $5 b$ ). The last coformer of this group is MLN, whose structure is similar to BZMDFMA. Also having $Z^{\prime}=0.5$, MLN lies in a twofold axis and is bonded to equivalent BZMD molecules by an $\mathrm{Im} \cdots \mathrm{H}-\mathrm{O}$ interaction (1.9 and $2.3 \AA$ ), giving rise to the same crystal packing. Notice that the carboxylic acid group is disordered between two orientations and, as a consequence, the corresponding $\mathrm{H}$ atom cannot be unequivocally located from the difference maps. However, two facts support the classification of BZMDMLN as a cocrystal. First, no residual charges were observed near the $\mathrm{N}$ atom of the imidazole group. Furthermore, the $\mathrm{C}-\mathrm{O}$ distances of both components of the disordered model have values which can be associated with a protonated carboxylic acid group, as will be discussed later.

3.1.3. Triple donor coformers. Considering the success of our crystal-design strategy to produce 2:1 cocrystals, the next step was to test coformers with three hydroxy groups in order to reach a 3:1 stoichiometry. Thus, new multicomponent forms were crystallized using 2,6DBA and 3,5DBA (Fig. 6).

BZMD2,6DBA (Fig. $6 a$ ) has three binding sites, but just one of them forms an intermolecular interaction involving the projected synthon. The remaining hydroxy groups are beside the carbonyl moiety and, as may be expected from the previous results, they form intramolecular hydrogen bonds

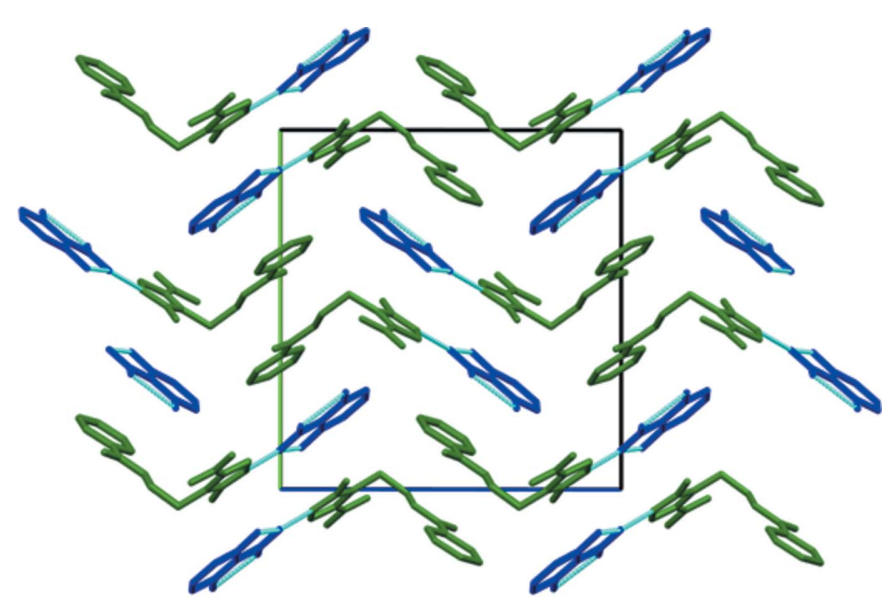

(a)

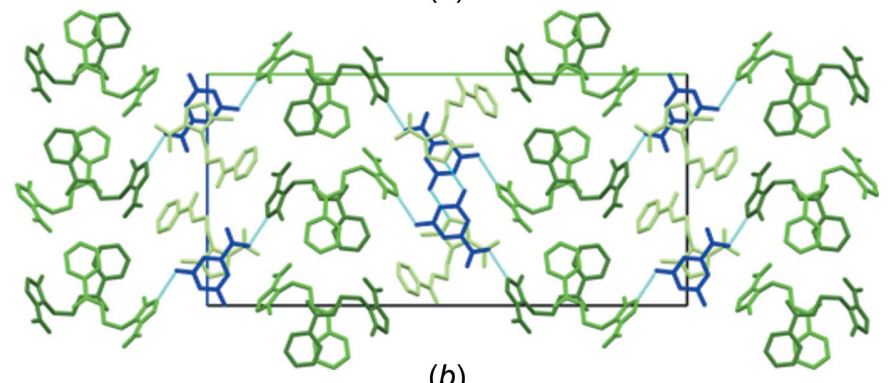

Figure 6

(b)

Packing of $(a)$ the salt BZMD2,6DBA and $(b)$ the cocrystal BZMD3,5DBA along the $a$ axis. $\mathrm{H}$ atoms have been omitted for clarity. BZMD is represented in green and coformers in blue. Lattice axes $a, b$ and $c$ are colour-coded red, green and blue, respectively. 


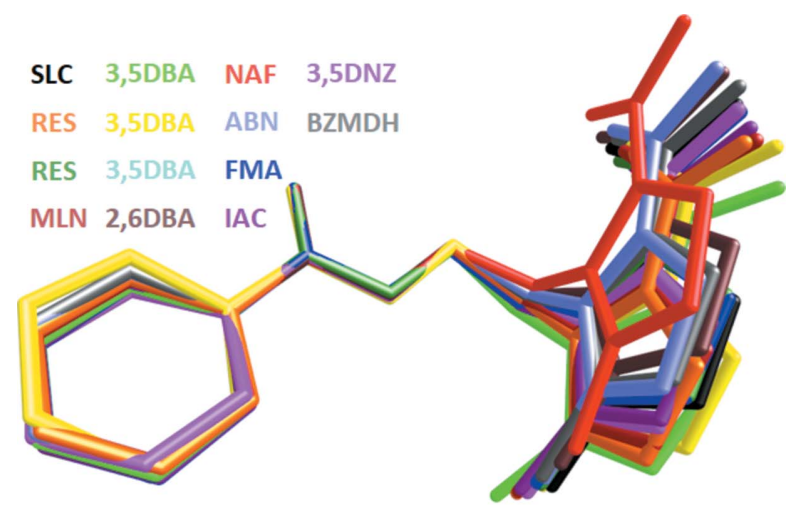

(a)

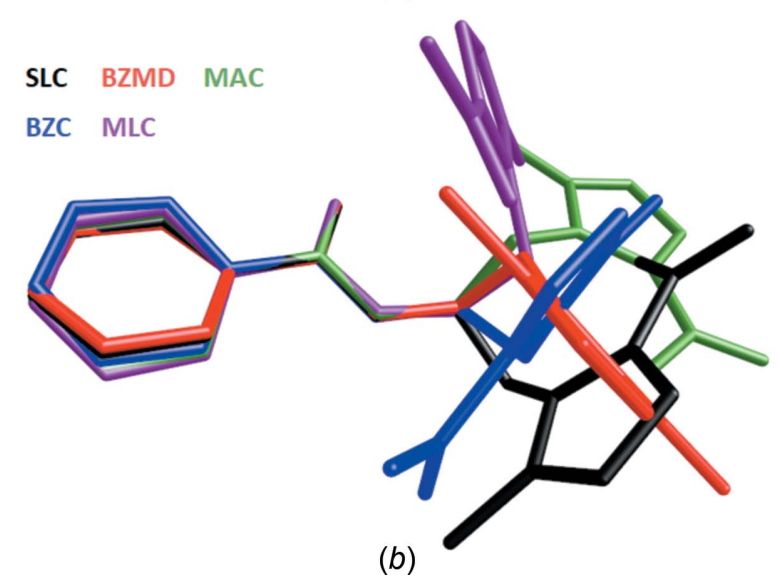

Figure 7

Comparison of the observed BZMD molecular conformations overlapping the ethyl groups and colour labelled based on the corresponding conformer. $\mathrm{H}$ atoms have been omitted for clarity.

blocking the Im $\cdots \mathrm{H}-\mathrm{O}$ interactions. Regarding the target synthon, the refined position places the $\mathrm{H}$ atom approximately at the middle [1.24 (5) $\AA$ ] of the donor-acceptor distance [2.564 (2) $\AA$ ], closer to the imidazole group. This configuration could be classified as a salt, but it will be discussed in detail later. The crystalline packing was based on the alternation between the coformer and API molecules in a zigzag pattern. Due to the greater affinity for intramolecular interactions in the coformer, the BZMD2,6DBA stoichiometry is 1:1, despite the availability of three binding sites.

According to our proposal, the asymmetric unit of BZMD3,5DBA (Fig. 6b) consists of three molecules of BZMD and one molecule of 3,5DBA, with the hydroxy groups actively participating in the proposed intermolecular hydrogen bonds, confirming a 3:1 stoichiometry based purely on the Im-HO synthon [1.73 (4), 1.78 (4) and 1.83 (4) $\AA$ ]. The crystal packing resembles those of the 2:1 structures, but the third BZMD molecule is intercalated in the coformer column. The last coformer combines several of the key concepts addressed in our crystal-design strategy. First, the Im $\cdots \mathrm{H}-\mathrm{O}$ interaction is a reliable synthon for supramolecular structures, where the hydroxy group can be provided by molecules containing phenol and/or carboxylic acid groups. Several binding sites can be used to reach higher API-coformer stoichiometries, but intramolecular hydrogen bonds and/or
Table 2

Selected dihedral angles $\left({ }^{\circ}\right)$ of the BZMD molecule in single and multicomponent solid forms.

The $\theta$ column lists the angles between the planes defined by the imidazole (BZMD) and phenyl/carboxylic (coformer) groups.

\begin{tabular}{|c|c|c|c|c|c|c|c|}
\hline & & $\varphi_{1}$ & $\varphi_{2}$ & $\varphi_{3}$ & $\varphi_{4}$ & $\varphi_{5}$ & $\theta$ \\
\hline 1 & BZMD & 104.1 & -63.9 & -94.9 & 178.6 & -10.6 & - \\
\hline 2 & BZMDH & 100.9 & -66.4 & -178.4 & 179.5 & -6.3 & - \\
\hline 3 & SLC & 111.0 & -70.2 & -170.8 & 177.4 & 7.3 & 3.8 \\
\hline 4 & $3,5 \mathrm{DBA}$ & 110.3 & -67.7 & -169.5 & -168.7 & -11.8 & 5.7 \\
\hline 5 & RES & 106.6 & -59.8 & -174.0 & -171.8 & 6.5 & 30.3 \\
\hline 6 & NAF & 105.6 & -68.8 & 170.5 & 178.3 & 9.0 & 6.9 \\
\hline 7 & $3,5 \mathrm{DBA}$ & 105.4 & -63.4 & -159.1 & 179.4 & $7.4 / 10.9$ & 83.7 \\
\hline 8 & RES & 105.1 & -71.5 & -169.1 & 175.6 & 3.7 & 31.6 \\
\hline 9 & $3,5 \mathrm{DBA}$ & 104.0 & -63.6 & -171.5 & -178.6 & 17.8 & 89.9 \\
\hline 10 & ABN & 103.6 & -62.5 & 178.6 & -179.1 & -3.1 & 13.2 \\
\hline 11 & FMA & 103.3 & -70.5 & -169.6 & 175.9 & 3.8 & 22.6 \\
\hline 12 & MLN & 102.9 & -61.5 & -173.2 & -172.9 & 7.5 & $22.8 / 29.3$ \\
\hline 13 & $2,6 \mathrm{DBA}$ & 102.4 & -73.5 & -177.2 & 178.4 & 3.4 & 5.5 \\
\hline 14 & $3,5 \mathrm{DNZ}$ & 99.8 & -65.5 & -173.9 & -178.0 & 20.2 & 75.4 \\
\hline 15 & IAC & 99.8 & -65.9 & -168.4 & 177.1 & 4.4 & 20.4 \\
\hline 16 & MAC & 94.5 & -171.0 & 81.6 & 171.2 & -10.4 & 5.1 \\
\hline 17 & $\mathrm{BZC}$ & -82.9 & -63.0 & 159.2 & -178.2 & -11.0 & 17.5 \\
\hline 18 & MLC & -72.7 & -66.7 & 98.1 & 170.2 & -14.7 & 59.9 \\
\hline
\end{tabular}

coformer dimers could limit the available coformers. The first problem can be addressed by selecting appropriate molecular structures, whereas the competition between coformer homosynthons and $\mathrm{Im} \cdots \mathrm{H}-\mathrm{O}$ interactions should be solved by serendipity.

\section{Discussion}

After presenting several new solid forms of BZMD, it is interesting to perform a comparative analysis between these structures. As a starting point, the molecular conformation of the API molecule will be considered. Comparing the observed BZMD molecular conformations (Fig. 7), it may be concluded that the main changes are related to the orientation among the phenyl, ethyl formate and imidazole groups. Table 2 lists the backbone dihedral angles of the BZMD molecule for all the structures reported in this contribution following the notation of Fig. 1. By overlapping the ethyl formate groups, it is possible to notice that the structures differ mainly by rotations of the imidazole and phenyl moieties. In particular, the phenyl group is always slightly out of the plane of the ethyl formate chain $\left(\left|\varphi_{5}\right|<20^{\circ}\right)$. This conformational flexibility does not seem to be determinant for the crystal packing, but a mechanism to optimize API-API interactions in the BZMD columns (see, for example, Fig. 5). Considering the imidazole group and following the numbering of Table 2, the set of crystalline structures $\mathbf{3}$ to $\mathbf{1 5}$ (Fig. $7 a$ ) exhibit a continuous change in the dihedral angle associated with the orientation of this moiety $\left(\varphi_{1}\right)$. Notice that BZMDH can be included in this set but not BZMD, showing that this conformation is favourable for the crystal packing of multicomponent forms. The largest dihedral angle associated with the orientation of the imidazole ring $\left(\varphi_{1}\right)$ corresponds to BZMDSLC $\left(111^{\circ}\right)$ and the smallest to BZMDIAC $\left(99.8^{\circ}\right)$. It is also interesting to observe that all the non-equivalent molecules of the higher stoichio- 
metry forms (BZMDRES and BZMD3,5DBA) lie between these limits. Finally, BZMD and three cocrystals (16, 17 and 18) differ considerably from the previous set and need to be considered separately, as shown in Fig. 7(b), where BZMDSLC (3) was included as a reference. The characteristic feature of this set is the flipping of the methylnitroimidazole group compared with BZMDSLC. Besides that, they have larger rotations of the imidazole group, which also induce additional torsion in the ethyl formate chain $\left(\varphi_{2}\right.$ and $\left.\varphi_{3}\right)$.

The conformational changes of the BZMD molecules are directly related to the backbone of the supramolecular structure defined by the API. In the Results (\$3) section, several structures have been described as being formed by BZMD columns/layers with intercalated coformers. The key interaction in this packing is the $\pi$-stacking of the BZMD molecules (see Table S3 in the supporting information). Two kinds of $\pi-\pi$ interactions between BZMD molecules were observed in cocrystal/salts, i.e. imidazole-phenyl and phenyl-phenyl, and only BZMDH exhibits an imidazole-imidazole interaction. Despite the involved rings, all the $\pi-\pi$ interactions can be classified as approximately parallel $\left(\alpha<20^{\circ}\right)$, but not facecentred (offset $>1.3 \AA$ ) (Martinez \& Iverson, 2012). Representative interactions are shown in Fig. 8 evidenced by the BZMD Hirshfeld surface mapped with the shape index, which exhibits a 'bow-tie' pattern of red-blue triangles fingerprinting the $\pi-\pi$ interactions (McKinnon et al., 2004; Spackman \& Jayatilaka, 2009). Imidazole-phenyl interactions are observed with the BZC, MAC and DNZ coformers (Fig. 2), but, in BZMDBZC and BZMDMAC, the API forms dimers intercalated with the coformers. The $\pi$-stacked columns are present in BZMD3,5DNZ, which resemble the columns in Fig. 5 defined by phenyl-phenyl interactions. In fact, the stacking of the phenyl rings is the most prevalent backbone in the BZMD multicomponent forms, being observed with the coformers SLC, MLC, IAC (Fig. 4), RES, FMA, MLN (Fig. 5) and 3,5DBA (Fig. 6). The $\pi$-stacking is also evident in the alternative projection shown in Fig. 4 for MLC and IAC. In the remaining structures, i.e. BZMDABN, BZMDNAF and BZMD2,6DBA, the aromatic rings of the coformers determine the supramolecular organization by API-coformer interactions. These kinds of interactions are also observed in several of the previously described structures, but the APIAPI interaction plays a key role in the crystal packing (see Table S3 in the supporting information). It is also interesting to notice that the molecular conformation shown in Fig. 7(a) really favours the $\pi$-stacking and, consequently, multicomponent BZMD forms.

Regarding the API-coformer Im-HO synthon, the first point to be considered is the charge transfer between these molecules. According to the $\mathrm{p} K_{\mathrm{a}}$ rule, based on the probability of proton transfer between the components in a reaction, the $\mathrm{p} K_{\mathrm{a}}$ difference $\left[\Delta \mathrm{p} K_{\mathrm{a}}=\mathrm{p} K_{\mathrm{a}}(\right.$ protonated base $)-\mathrm{p} K_{\mathrm{a}}($ acid $\left.)\right]$ can be used as a predictor of the formation of a cocrystal/salt (Delori et al., 2013). Based on a detailed statistical analysis, Cruz-Cabeza (2012) identified three types of behaviour: (i) where cocrystals are almost exclusively formed $\left(\Delta K_{\mathrm{a}}<-1\right)$, (ii) where salts are prevalent $\left(\Delta K_{\mathrm{a}}>4\right)$ and (iii) a co-existence region where both salts and cocrystals can be observed $(-1 \leq$ $\left.\Delta K_{\mathrm{a}} \leq 4\right)$. In the co-existence region, complex proton behaviours can be observed not allowing the classification of those structures under one of the proton-transfer conditions, giving rise to the 'salt-cocrystal continuum' (Childs et al., 2011; da Silva et al., 2013). In good agreement with this rule, nine of the conformers give rise to cocrystals, once the difference between the $\mathrm{p} K_{\mathrm{a}}$ of the drug and coformer is lower than -1 (see Table S4 in the supporting information). The remaining APIcoformer pairs are in the co-existence region, with most of them having $\Delta \mathrm{p} K_{\mathrm{a}}<0$ and being identified as cocrystals. Two cases need to be considered separately, $\operatorname{BZMDMLC}\left(\Delta \mathrm{p} K_{\mathrm{a}}=\right.$ $0.72)$ and BZMD2,6DBA $\left(\Delta \mathrm{p} K_{\mathrm{a}}=-0.22\right)$, because the corresponding crystalline structures cannot be described as cocrystals. In the case of BZMDMLC, the proton was located and refined based on the difference Fourier maps, placing it close to the imidazole group characterizing a salt. Considering the linear relationship established by Cruz-Cabeza (2012), the BZMD/MLC pair has a probability of $40.2 \%$ of crystallizing as a salt. On the other hand, in the BZMD/2,6DBA system, this probability is $24.3 \%$, which is also the highest probability observed among the BZMD cocrystals reported here. The

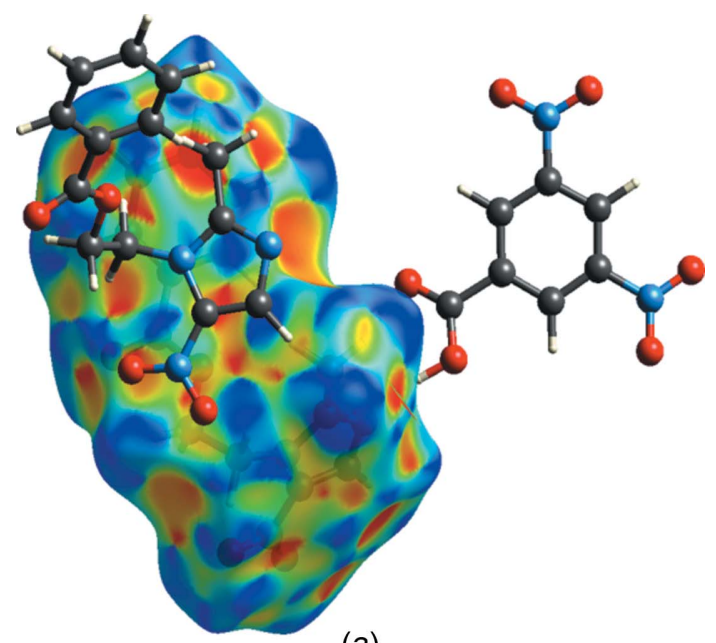

(a)

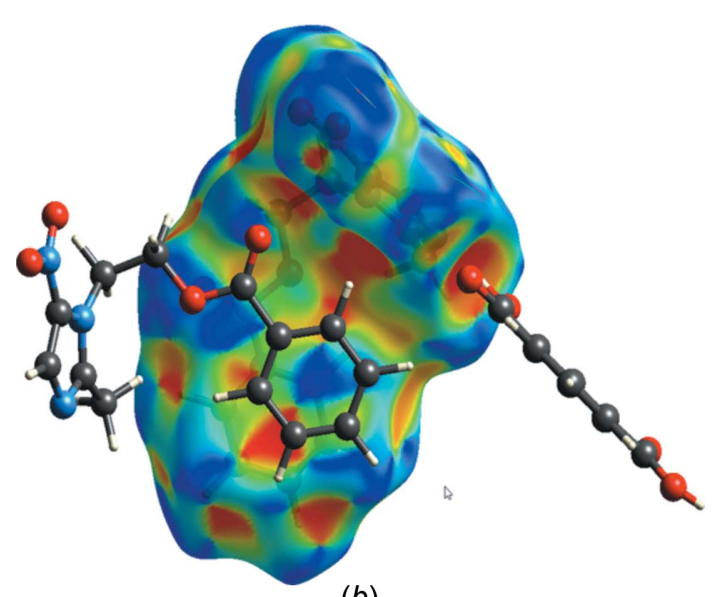

(b)

Figure 8

Hirshfeld surfaces mapped with the shape index of (a) BZMDBZC and (b) BZMDIAC, showing the $\pi$-stacking of the BZMD columns. 

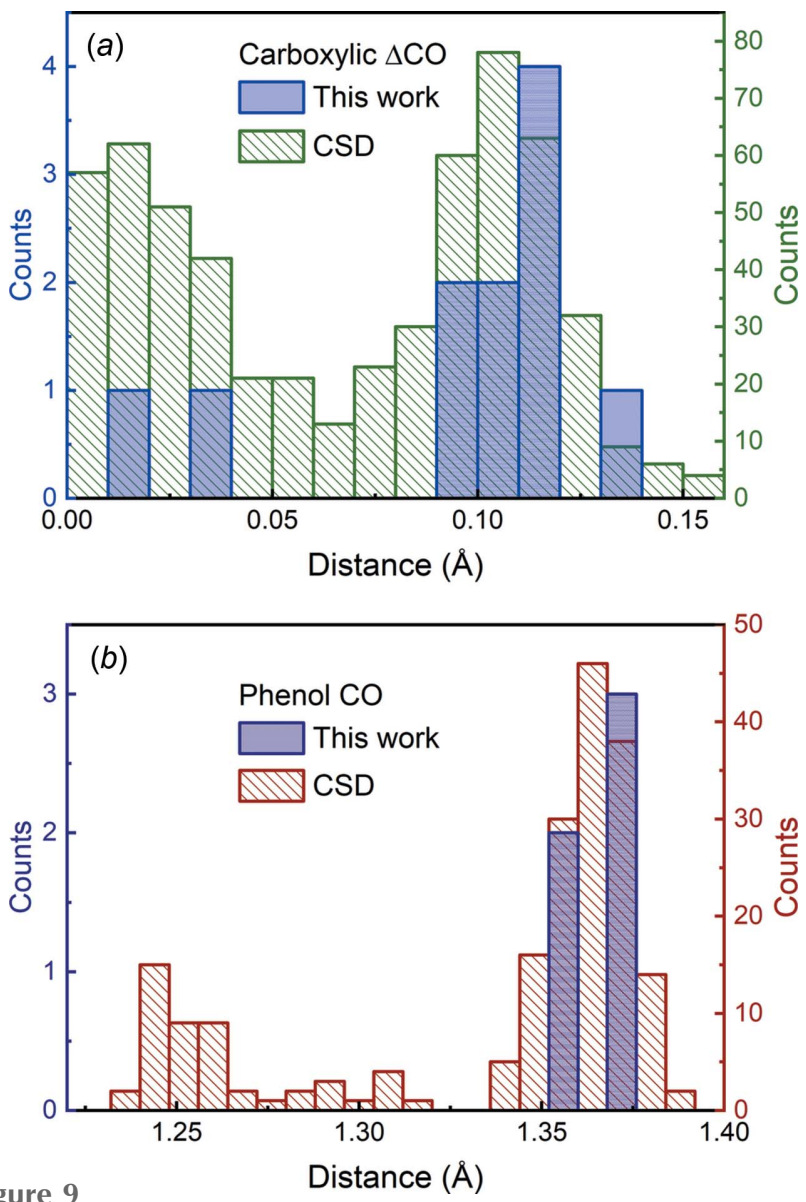

Figure 9

(a) Distribution of the difference between the $\mathrm{C}-\mathrm{O}$ distances of the carboxylic acid group. (b) Distribution of the $\mathrm{C}-\mathrm{O}$ distances of the phenol groups.

proton position refinement placed it slightly closer to the imidazole group, but with the largest donor-hydrogen distance $(1.24 \AA)$. Even though several of the structures reported here could be predicted based on the $\Delta \mathrm{p} K_{\mathrm{a}}$ rule, from the point of view of the proper classification of multicomponent forms as cocrystals or salts, other markers can be used. Based on the proposed synthon, the determined structures were compared with a search performed in the CSD for the Im-carboxylic and Im-phenol synthons. The hits related to carboxylic acid were processed calculating the difference $(\triangle \mathrm{CO})$ between the two $\mathrm{C}-\mathrm{O}$ bond lengths. It is well known that a $\Delta \mathrm{CO}$ lower than $\sim 0.03 \AA$ indicates that the carboxylic acid group is deprotonated. The analysis of the Im-carboxylic acid hits agrees with this rule as two distributions are observed (Fig. 9a), which are centred around $\Delta \mathrm{CO}=\sim 0.105$ and $\sim 0.0 \AA$, being related to protonated and deprotonated carboxylic acid groups, respectively. Comparing this distribution with the BZMD salts/cocrystals, one may conclude that all the $\triangle \mathrm{CO}$ cocrystals lie in the expected region. In the case of the salts, the lowest hit $(\triangle \mathrm{CO}=0.016 \AA)$ corresponds to BZMDMLC, which is in good agreement with the salt character predicted by the $\Delta \mathrm{p} K_{\mathrm{a}}$ rule. Just above this hit, there is that from BZMD2,6DBA $(\triangle \mathrm{CO}=0.0354 \AA)$, which is in the co-existence region of the $\Delta \mathrm{p} K_{\mathrm{a}}$ rule, but lies around the upper limit of the deprotonated region in the $\Delta \mathrm{CO}$ plot, confirming that this multicomponent form was correctly classified as a salt. For the case of the Im-phenol synthon, the statistical distribution of $\mathrm{C}-\mathrm{O}$ distances is shown in Fig. 9(b). These distances are mainly distributed around two maxima at 1.25 and $1.36 \AA$, which are related to deprotonated and protonated hydroxy groups, respectively. As expected, the $\mathrm{C}-$ $\mathrm{O}$ distances of the BZMD cocrystals based on phenols are in the range of the protonated hydroxy distances, confirming the previous classification.

The previous results clearly confirm the reliability of the Im-carboxylic and Im-phenol synthons as a crystal-design strategy to produce multicomponent forms with variable stoichiometry. Comparing the crystalline structures, three synthon geometries were identified and the Im-phenol synthon was observed with the coformers RES, NAF and 3,5DBA. On the other hand, the simple Im-carboxylic acid synthon, with and without charge transfer, is present in forms containing ABN, IAC, FMA, MLC, MLN, SLC, 2,6DBA and 3,5DNZ. A third synthon derived from Im-carboxylic acid forms an $R_{2}^{2}(8)$ ring including the methyl moiety of the methylnitroimidazole group. BZC and MAC use the last synthon to stabilize their crystalline structures. These synthons can also be analyzed by comparing the acceptor-donor distances of the structures reported in this work with those in
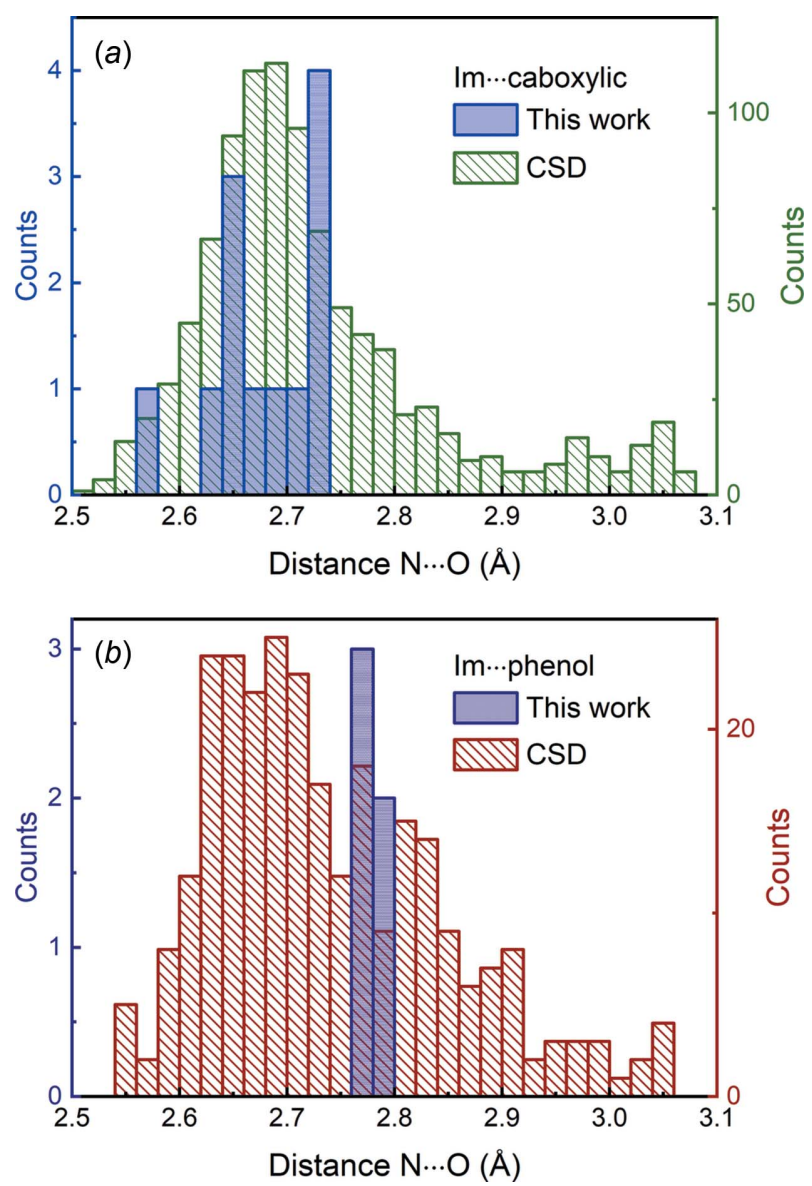

Figure 10

Comparison of the distribution of the acceptor-donor distances of the synthons based on (a) carboxylic acid and $(b)$ phenol groups. 
the CSD. Fig. 10 shows the histograms of the $\mathrm{N}-\mathrm{O}$ distances when carboxylic acid or phenol groups are considered. Both synthons have well-defined distributions spanning approximately the same value range, with that related to the phenol group being a little wider. The acceptor-donor distances calculated from the BZMD new forms lie within the general distribution ranges, despite the fact that the phenol-based cocrystals exhibit distances slightly longer than the distribution maxima. Another interesting parameter is the angle $(\theta)$ between the plane containing the imidazole ring and either that defined by the phenyl or the carboxylic acid group. Despite which synthon is used, there is no clear relationship among the angles listed in Table 2, showing that the proposed synthon does not restrict the relative orientation of the API and coformer, giving the new forms plenty of flexibility to optimize the supramolecular organization.

\section{Conclusion}

This work was aimed at rationally designing multicomponent forms of BZMD. To achieve this goal, a target supramolecular synthon was selected on the basis of the BZMD molecular structure and a statistical analysis of the Cambridge Structural Database. Thus, the imidazole group of BZMD seems to be a suitable acceptor for hydrogen bonds with coformers containing hydroxy groups. In order to explore different stoichiometries from the same synthon (1:1, 2:1 and 3:1), we choose coformers with carboxylic acid and phenol groups, based on the available binding sites. This strategy generated thirteen new solid forms (two salts and eleven cocrystals). At first, coformers with a single hydroxy donor (BZC, NAF, MAC, 3,5DNZ and ABN) were selected and multicomponent structures with a 1:1 stoichiometry were produced, proving that the proposed strategy was successful. Coformers containing two binding sites were tested in order to explore solid forms with a 2:1 stoichiometry. In this sense, carboxylic acids and phenols with donor groups were selected, but despite the fact that these coformers have two hydroxy groups, several of them crystallize with a 1:1 stoichiometry. It was observed that two effects could limit synthon formation. In coformers with close hydroxy groups (SLC and MLC), intramolecular hydrogen bonds are more favourable than the ImHO synthon. Furthermore, coformer homosynthons (IAC) could also reduce the number of active sites available to be linked to the API. Based on these observations, the molecular geometry of the coformer was chosen in order to avoid intramolecular hydrogen bonds and three cocrystals with a 2:1 stoichiometry were produced (RES, FMA and MLN). The next step was to test coformers with three hydroxy groups in order to reach a 3:1 stoichiometry. Thus, new multicomponent forms were crystallized using 2,6DBA and 3,5DBA. The former has intramolecular hydrogen bonds limiting the stoichiometry to $1: 1$, but a $3: 1$ ratio was achieved with $3,5 \mathrm{DBA}$, combining carboxylic and phenol synthons. When compared with other hits in the CSD, the intermolecular parameters of the Im-HO synthon in the BZMD cocrystals/salts are similar, showing that this is a robust synthon. In addition, the lack of a geometrical constraint in the relative orientation of the API and coformer provides additional flexibility to the selected interaction, confirming that $\mathrm{Im}-\mathrm{HO}$ is a trustworthy synthon for use in a crystal-design strategy.

\section{Funding information}

Funding for this research was provided in part by: the Brazilian agencies CAPES (Finance Code 001), FUNCAP (PPSUS and PRONEX PR2-0101-00006.01.00/15), and CNPq.

\section{References}

Aitipamula, S., Chow, P. S. \& Tan, R. B. H. (2014). CrystEngComm, 16, 3451-3465.

Babu, N. J., Sanphui, P. \& Nangia, A. (2012). Chem. Asian J. 7, $2274-$ 2285.

Barikah, K. Z. (2018). Syst. Rev. Pharm. 9, 79-82.

Bempong, D. K., Manning, R. G., Mirza, T. \& Bhattacharyya, L. (2005). J. Pharm. Biomed. Anal. 38, 776-780.

Berry, D. J. \& Steed, J. W. (2017). Adv. Drug Deliv. Rev. 117, 3-24.

Blagden, N., de Matas, M., Gavan, P. T. \& York, P. (2007). Adv. Drug Deliv. Rev. 59, 617-630.

Bolla, G. \& Nangia, A. (2016). Chem. Commun. 52, 8342-8360.

Bruker (2012). APEX3. Bruker AXS Inc., Madison, Wisconsin, USA.

Bruker (2016). SAINT and SADABS. Bruker AXS Inc., Madison, Wisconsin, USA.

Caira, M. R., Nassimbeni, L. R. \& van Oudtshoorn, B. (1993). J. Pharm. Sci. 82, 1006-1009.

Cerreia Vioglio, P., Chierotti, M. R. \& Gobetto, R. (2017). Adv. Drug Deliv. Rev. 117, 86-110.

Childs, S. L., Stahly, G. P. \& Park, A. (2011). Mol. Pharm. 4, 323-338.

Corpinot, M. K. \& Bučar, D.-K. (2018). Cryst. Growth Des. 19, 14261453.

Cruz-Cabeza, A. J. (2012). CrystEngComm, 14, 6362-6365.

Dalpiaz, A., Pavan, B. \& Ferretti, V. (2017). Drug Discov. Today, 22, 1134-1138.

Delori, A., Friščić, T. \& Jones, W. (2012). CrystEngComm, 14, 23502362.

Delori, A., Galek, P. T. A., Pidcock, E., Patni, M. \& Jones, W. (2013). CrystEngComm, 15, 2916-2928.

Desiraju, G. R. (2010). J. Chem. Sci. 122, 667-675.

Diniz, L. F., Souza, M. S., Carvalho, P. S. Jr, da Silva, C. C. P., D’Vries, R. F. \& Ellena, J. (2018). J. Mol. Struct. 1153, 58-68.

Do, J. L. \& Friscic, T. (2017). ACS Cent. Sci. 3, 13-19.

Dolomanov, O. V., Bourhis, L. J., Gildea, R. J., Howard, J. A. K. \& Puschmann, H. (2009). J. Appl. Cryst. 42, 339-341.

Douroumis, D., Ross, S. A. \& Nokhodchi, A. (2017). Adv. Drug Deliv. Rev. 117, 178-195.

EMA (2015). Reflection Paper on the Use of Cocrystals of Active Substances in Medicinal Products. https://www.ema.europa.eu/en/.

FDA (2018). Guidance for Industry - Regulatory Classification of Pharmaceutical Cocrystals. https://www.fda.gov/.

Groom, C. R., Bruno, I. J., Lightfoot, M. P. \& Ward, S. C. (2016). Acta Cryst. B72, 171-179.

Hasa, D. \& Jones, W. (2017). Adv. Drug Deliv. Rev. 117, 147-161.

Healy, A. M., Worku, Z. A., Kumar, D. \& Madi, A. M. (2017). $A d v$. Drug Deliv. Rev. 117, 25-46.

Hoelgaard, A. \& Møller, N. (1983). Int. J. Pharm. 15, 213-221.

James, S. L., Adams, C. J., Bolm, C., Braga, D., Collier, P., Friščić, T., Grepioni, F., Harris, K. D., Hyett, G., Jones, W., Krebs, A., Mack, J., Maini, L., Orpen, A. G., Parkin, I. P., Shearouse, W. C., Steed, J. W. \& Waddell, D. C. (2012). Chem. Soc. Rev. 41, 413-447.

Kavanagh, O. N., Croker, D. M., Walker, G. M. \& Zaworotko, M. J. (2019). Drug Discov. Today, 24, 796-804. 
Macrae, C. F., Bruno, I. J., Chisholm, J. A., Edgington, P. R., McCabe, P., Pidcock, E., Rodriguez-Monge, L., Taylor, R., van de Streek, J. \& Wood, P. A. (2008). J. Appl. Cryst. 41, 466-470.

Martinez, C. R. \& Iverson, B. L. (2012). Chem. Sci. 3, 2191-2201.

McKinnon, J. J., Spackman, M. A. \& Mitchell, A. S. (2004). Acta Cryst. B60, 627-668.

Pedretti, A., Villa, L. \& Vistoli, G. (2004). J. Comput. Aided Mol. Des. 18, 167-173.

Peraka, K. S., Lusi, M., Bajpai, A. \& Zaworotko, M. J. (2017). Cryst. Growth Des. 17, 959-962.

Saha, S. \& Desiraju, G. R. (2018). J. Am. Chem. Soc. 140, 63616373.

Sarkar, A. \& Rohani, S. (2015). J. Pharm. Sci. 104, 98-105.

Sarraguça, M. C., Paisana, M., Pinto, J. \& Lopes, J. A. (2016). Eur. J. Pharm. Sci. 90, 76-84.
Shaikh, R., Singh, R., Walker, G. M. \& Croker, D. M. (2018). Trends Pharmacol. Sci. 39, 1033-1048.

Shayanfar, A. \& Jouyban, A. (2014). Powder Technol. 262, 242-248. Shayanfar, A., Velaga, S. \& Jouyban, A. (2014). Fluid Phase Equilib. 363, 97-105.

Sheldrick, G. M. (2015a). Acta Cryst. A71, 3-8.

Sheldrick, G. M. (2015b). Acta Cryst. C71, 3-8.

Silva, C. C. P. da, de Oliveira, R., Tenorio, J. C., Honorato, S. B., Ayala, A. P. \& Ellena, J. (2013). Cryst. Growth Des. 13, 4315-4322.

Spackman, M. A. \& Jayatilaka, D. (2009). CrystEngComm, 11, 19-32. Spek, A. L. (2009). Acta Cryst. D65, 148-155.

Spek, A. L. (2015). Acta Cryst. C71, 9-18.

Wang, T., Stevens, J. S., Vetter, T., Whitehead, G. F. S., VitoricaYrezabal, I. J., Hao, H. \& Cruz-Cabeza, A. J. (2018). Cryst. Growth Des. 18, 6973-6983. 


\section{supporting information}

Acta Cryst. (2019). C75, 483-495 [https://doi.org/10.1107/S2053229619003838]

\section{The design of novel metronidazole benzoate structures: exploring stoichiometric diversity}

\section{Yara Santiago de Oliveira, Wendell Saraiva Costa, Poliana Ferreira Borges, Maria Silmara Alves de Santana and Alejandro Pedro Ayala}

\section{Computing details}

For all structures, data collection: APEX3 (Bruker, 2012); cell refinement: SAINT (Bruker, 2016); data reduction: SAINT (Bruker, 2016); program(s) used to solve structure: SHELXT2014 (Sheldrick, 2015a); program(s) used to refine structure: SHELXL2018 (Sheldrick, 2015b); molecular graphics: OLEX2 (Dolomanov et al., 2009), Mercury (Version 3.10; Macrae et al., 2008) and VEGA (Pedretti et al., 2004); software used to prepare material for publication: OLEX2 (Dolomanov et al., 2009).

2-(2-Methyl-5-nitro-1H-imidazol-1-yl)ethyl benzoate-benzoic acid (1/1) (bzmolbzc)

Crystal data

$\mathrm{C}_{13} \mathrm{H}_{13} \mathrm{~N}_{3} \mathrm{O}_{4} \cdot \mathrm{C}_{7} \mathrm{H}_{6} \mathrm{O}_{2}$

$M_{r}=397.38$

Monoclinic, $P 2_{1} / n$

$a=13.5997(6) \AA$

$b=9.0354(4) \AA$

$c=16.5974(8) \AA$

$\beta=108.646(2)^{\circ}$

$V=1932.42(15) \AA^{3}$

$Z=4$

Data collection

Bruker APEXII CCD

diffractometer

$\varphi$ and $\omega$ scans

Absorption correction: multi-scan

(SADABS; Bruker, 2016)

$T_{\min }=0.675, T_{\max }=0.753$

60527 measured reflections

\section{Refinement}

Refinement on $F^{2}$

Least-squares matrix: full

$R\left[F^{2}>2 \sigma\left(F^{2}\right)\right]=0.045$

$w R\left(F^{2}\right)=0.130$

$S=1.07$

3542 reflections

339 parameters

0 restraints
$F(000)=832$

$D_{\mathrm{x}}=1.366 \mathrm{Mg} \mathrm{m}^{-3}$

$\mathrm{Cu} K \alpha$ radiation, $\lambda=1.54178 \AA$

Cell parameters from 9997 reflections

$\theta=5.1-68.0^{\circ}$

$\mu=0.86 \mathrm{~mm}^{-1}$

$T=300 \mathrm{~K}$

Prism, clear light colourless

$0.8 \times 0.2 \times 0.2 \mathrm{~mm}$

3542 independent reflections 2669 reflections with $I>2 \sigma(I)$

$R_{\text {int }}=0.053$

$\theta_{\max }=68.3^{\circ}, \theta_{\min }=3.7^{\circ}$

$h=-16 \rightarrow 16$

$k=-10 \rightarrow 10$

$l=-19 \rightarrow 20$

Hydrogen site location: difference Fourier map

All H-atom parameters refined

$w=1 /\left[\sigma^{2}\left(F_{\mathrm{o}}^{2}\right)+(0.0462 P)^{2}+0.7992 P\right]$

where $P=\left(F_{\mathrm{o}}^{2}+2 F_{\mathrm{c}}^{2}\right) / 3$

$(\Delta / \sigma)_{\max }<0.001$

$\Delta \rho_{\max }=0.16$ e $\AA^{-3}$

$\Delta \rho_{\min }=-0.14$ e $\AA^{-3}$ 
Extinction correction: SHELXL2018

(Sheldrick, 2015b),

$\mathrm{Fc}^{*}=\mathrm{kFc}\left[1+0.001 \times \mathrm{xc}^{2} \lambda^{3} / \sin (2 \theta)\right]^{-1 / 4}$

Extinction coefficient: 0.0027 (4)

\section{Special details}

Geometry. All esds (except the esd in the dihedral angle between two 1.s. planes) are estimated using the full covariance matrix. The cell esds are taken into account individually in the estimation of esds in distances, angles and torsion angles; correlations between esds in cell parameters are only used when they are defined by crystal symmetry. An approximate (isotropic) treatment of cell esds is used for estimating esds involving l.s. planes.

Refinement. Single-crystal X-ray diffraction data collection ( scans and $\omega$ scans with $\kappa$ and $\theta$ offsets) were performed at room temperature on a Bruker D8 VENTURE equipped with a Kappa goniometer, PHOTON II CPAD detector, a Mo $K \alpha$ INCOATEC I $\mu \mathrm{S} 3.0$ microfocus source $(\lambda=0.71073 \AA)$. The crystal centring, unit-cell determination, refinement of the unit-cell parameters and data collection was controlled through the program APEX3 (Bruker, 2012). The frame integration was performed using SAINT (Bruker, 2016) and the intensities were scaled and absorption corrected using SADABS (Bruker, 2001). Using OLEX2 (Dolomanov et al., 2009), the structure was solved by intrinsic phasing using SHELXT (Sheldrick, 2015) and refined by full-matrix least-squares calculation based on $F^{2}$ for all reflection using SHELXL (Sheldrick, 2007). All non-H atoms were refined anisotropically.

Fractional atomic coordinates and isotropic or equivalent isotropic displacement parameters $\left(\hat{A}^{2}\right)$

\begin{tabular}{|c|c|c|c|c|}
\hline & $x$ & $y$ & $z$ & $U_{\text {iso }} * / U_{\text {eq }}$ \\
\hline $\mathrm{O} 3$ & $0.57161(11)$ & 0.97707 (18) & $0.14818(9)$ & $0.0723(4)$ \\
\hline $\mathrm{O}^{\prime}$ & $0.65654(13)$ & $0.60123(18)$ & $0.53629(11)$ & $0.0806(5)$ \\
\hline N1 & $0.62802(12)$ & $0.99073(17)$ & $0.32969(9)$ & $0.0568(4)$ \\
\hline $\mathrm{O} 2^{\prime}$ & $0.52139(13)$ & 0.69513 (19) & $0.56528(12)$ & $0.0887(5)$ \\
\hline N3 & $0.79953(14)$ & $0.9851(2)$ & $0.30460(11)$ & $0.0681(5)$ \\
\hline $\mathrm{O} 1$ & $0.87927(13)$ & $0.9118(2)$ & $0.31765(12)$ & $0.0933(5)$ \\
\hline N2 & $0.65260(13)$ & 0.80491 (19) & $0.42084(11)$ & $0.0655(5)$ \\
\hline $\mathrm{O} 4$ & $0.43536(12)$ & $0.8503(2)$ & $0.06689(11)$ & $0.0930(6)$ \\
\hline $\mathrm{O} 2$ & $0.78341(14)$ & $1.1005(2)$ & $0.26413(12)$ & $0.0933(6)$ \\
\hline $\mathrm{C} 1^{\prime}$ & $0.61614(14)$ & $0.4999(2)$ & $0.65137(12)$ & $0.0543(4)$ \\
\hline $\mathrm{C} 1$ & $0.72512(15)$ & $0.9308(2)$ & $0.34107(12)$ & $0.0584(5)$ \\
\hline $\mathrm{C} 8$ & $0.60097(15)$ & $0.8057(2)$ & $0.05160(12)$ & $0.0605(5)$ \\
\hline $\mathrm{C} 7^{\prime}$ & $0.59173(15)$ & $0.6076(2)$ & $0.57987(12)$ & $0.0587(5)$ \\
\hline $\mathrm{C} 3$ & $0.58695(16)$ & $0.9091(2)$ & $0.37957(12)$ & $0.0587(5)$ \\
\hline $\mathrm{C}^{\prime}$ & $0.54581(18)$ & $0.4792(2)$ & $0.69523(14)$ & $0.0660(5)$ \\
\hline $\mathrm{C} 2^{\prime}$ & $0.70976(16)$ & $0.4248(2)$ & $0.67683(15)$ & $0.0670(5)$ \\
\hline $\mathrm{C} 7$ & $0.52666(16)$ & $0.8765(3)$ & $0.08774(12)$ & $0.0642(5)$ \\
\hline $\mathrm{C} 2$ & $0.73912(17)$ & $0.8177(2)$ & $0.39708(14)$ & $0.0660(5)$ \\
\hline $\mathrm{C} 5$ & $0.5730(2)$ & $1.1090(2)$ & $0.27249(15)$ & $0.0692(6)$ \\
\hline $\mathrm{C} 9$ & $0.5671(2)$ & 0.6840 & $-0.00055(15)$ & $0.0765(6)$ \\
\hline $\mathrm{C} 4$ & $0.4827(2)$ & $0.9323(3)$ & $0.38706(18)$ & $0.0732(6)$ \\
\hline C6 & 0.50507 (19) & $1.0484(3)$ & $0.18877(14)$ & $0.0755(6)$ \\
\hline $\mathrm{C} 5^{\prime}$ & $0.5698(2)$ & $0.3854(3)$ & $0.76467(15)$ & $0.0746(6)$ \\
\hline C13 & $0.70145(18)$ & $0.8566(3)$ & $0.06717(15)$ & $0.0725(6)$ \\
\hline $\mathrm{C} 4^{\prime}$ & $0.6644(2)$ & $0.3150(3)$ & $0.79135(16)$ & $0.0786(7)$ \\
\hline $\mathrm{C} 12$ & $0.7663(2)$ & $0.7852(3)$ & $0.03012(18)$ & $0.0836(7)$ \\
\hline $\mathrm{C} 11$ & $0.7312(2)$ & $0.6645(3)$ & $-0.02131(17)$ & $0.0872(8)$ \\
\hline C3' & $0.7339(2)$ & $0.3331(3)$ & $0.74715(17)$ & $0.0807(7)$ \\
\hline
\end{tabular}




$\begin{array}{lllll}\text { C10 } & 0.6321(2) & 0.6133(3) & -0.03665(18) & 0.0900(8) \\ \text { H9 } & 0.497(2) & 0.656(3) & -0.0102(16) & 0.086(7)^{*} \\ \text { H12 } & 0.838(2) & 0.821(3) & 0.0425(18) & 0.107(9)^{*} \\ \text { H11 } & 0.774(2) & 0.614(3) & -0.0500(16) & 0.095(8)^{*} \\ \text { H2' } & 0.7584(18) & 0.435(3) & 0.6439(14) & 0.080(7)^{*} \\ \text { H4}^{\prime} & 0.679(2) & 0.249(3) & 0.8403(18) & 0.105(9)^{*} \\ \text { H6 }^{\prime} & 0.477(2) & 0.532(3) & 0.6729(15) & 0.092(8)^{*} \\ \text { H5' } & 0.520(2) & 0.375(3) & 0.7942(16) & 0.094(8)^{*} \\ \text { H3' } & 0.801(2) & 0.276(3) & 0.7636(18) & 0.111(9)^{*} \\ \text { H13 } & 0.7269(19) & 0.943(3) & 0.1044(16) & 0.087(8)^{*} \\ \text { H2 } & 0.8006(19) & 0.756(3) & 0.4194(15) & 0.083(7)^{*} \\ \text { H6A } & 0.450(2) & 0.972(3) & 0.1934(17) & 0.101(9)^{*} \\ \text { H6B } & 0.468(2) & 1.128(3) & 0.1501(17) & 0.098(8)^{*} \\ \text { H1' } & 0.647(2) & 0.681(4) & 0.494(2) & 0.138(12)^{*} \\ \text { H5A } & 0.6277(18) & 1.174(3) & 0.2639(15) & 0.081(7)^{*} \\ \text { H5B } & 0.5303(18) & 1.163(3) & 0.3013(15) & 0.079(7)^{*} \\ \text { H4A } & 0.474(2) & 0.864(3) & 0.4331(19) & 0.110(9)^{*} \\ \text { H4B } & 0.428(2) & 0.917(3) & 0.336(2) & 0.109(10)^{*} \\ \text { H4C } & 0.476(2) & 1.031(4) & 0.4081(17) & 0.102(9)^{*} \\ \text { H10 } & 0.608(2) & 0.526(4) & -0.072(2) & 0.123(11)^{*}\end{array}$

Atomic displacement parameters $\left(\AA^{2}\right)$

\begin{tabular}{|c|c|c|c|c|c|c|}
\hline & $U^{11}$ & $U^{22}$ & $U^{33}$ & $U^{12}$ & $U^{13}$ & $U^{23}$ \\
\hline $\mathrm{O} 3$ & $0.0652(9)$ & $0.0906(11)$ & $0.0624(8)$ & $-0.0003(8)$ & $0.0223(7)$ & $-0.0020(8)$ \\
\hline $\mathrm{O} 1^{\prime}$ & $0.0944(11)$ & $0.0741(10)$ & $0.0880(11)$ & $0.0183(8)$ & $0.0497(9)$ & $0.0230(9)$ \\
\hline N1 & $0.0661(10)$ & $0.0539(9)$ & $0.0508(8)$ & $0.0004(7)$ & $0.0194(7)$ & $0.0009(7)$ \\
\hline $\mathrm{O} 2^{\prime}$ & $0.0776(10)$ & $0.0867(12)$ & $0.1086(13)$ & $0.0266(9)$ & $0.0395(9)$ & $0.0356(10)$ \\
\hline N3 & $0.0712(11)$ & 0.0709 (11) & $0.0642(10)$ & $-0.0079(9)$ & $0.0246(9)$ & $-0.0045(9)$ \\
\hline $\mathrm{O} 1$ & $0.0775(11)$ & 0.0989 (13) & 0.1149 (14) & $0.0056(10)$ & $0.0466(10)$ & $0.0036(11)$ \\
\hline N2 & $0.0694(10)$ & $0.0638(10)$ & $0.0634(10)$ & $-0.0010(8)$ & $0.0213(8)$ & $0.0078(8)$ \\
\hline $\mathrm{O} 4$ & $0.0621(9)$ & $0.1282(15)$ & 0.0868 (11) & $-0.0115(10)$ & $0.0211(8)$ & -0.0059 (11) \\
\hline $\mathrm{O} 2$ & 0.0977 (12) & $0.0898(12)$ & 0.0998 (13) & $-0.0056(10)$ & $0.0421(10)$ & $0.0267(10)$ \\
\hline $\mathrm{C} 1^{\prime}$ & $0.0576(10)$ & $0.0471(10)$ & $0.0568(10)$ & $-0.0020(8)$ & $0.0164(8)$ & $-0.0019(8)$ \\
\hline $\mathrm{C} 1$ & $0.0622(11)$ & 0.0585 (11) & $0.0560(11)$ & $-0.0049(9)$ & $0.0211(9)$ & $-0.0028(9)$ \\
\hline $\mathrm{C} 8$ & $0.0641(11)$ & $0.0678(12)$ & $0.0490(10)$ & $-0.0042(9)$ & $0.0171(9)$ & $0.0126(9)$ \\
\hline$C 7^{\prime}$ & $0.0580(11)$ & $0.0538(11)$ & $0.0630(12)$ & $-0.0023(9)$ & $0.0173(9)$ & $0.0011(9)$ \\
\hline $\mathrm{C} 3$ & $0.0682(12)$ & $0.0574(11)$ & $0.0519(10)$ & $-0.0038(9)$ & $0.0211(9)$ & $-0.0028(9)$ \\
\hline $\mathrm{C} 6^{\prime}$ & $0.0706(13)$ & $0.0630(12)$ & 0.0693 (13) & $0.0057(10)$ & $0.0293(11)$ & $0.0018(10)$ \\
\hline $\mathrm{C} 2^{\prime}$ & $0.0599(12)$ & $0.0633(12)$ & $0.0773(14)$ & $0.0022(10)$ & $0.0211(10)$ & $0.0091(10)$ \\
\hline $\mathrm{C} 7$ & $0.0631(12)$ & $0.0762(14)$ & $0.0506(11)$ & $-0.0043(10)$ & $0.0144(9)$ & $0.0122(10)$ \\
\hline $\mathrm{C} 2$ & $0.0655(12)$ & $0.0642(12)$ & 0.0668 (13) & $0.0012(10)$ & $0.0192(10)$ & $0.0044(10)$ \\
\hline $\mathrm{C} 5$ & $0.0848(15)$ & $0.0568(12)$ & $0.0707(14)$ & $0.0127(11)$ & $0.0315(12)$ & $0.0103(10)$ \\
\hline C9 & $0.0744(15)$ & $0.0848(16)$ & $0.0674(13)$ & $-0.0092(13)$ & $0.0188(11)$ & $0.0021(12)$ \\
\hline $\mathrm{C} 4$ & $0.0734(14)$ & $0.0808(16)$ & $0.0712(15)$ & $0.0014(12)$ & $0.0311(12)$ & $0.0003(13)$ \\
\hline C6 & $0.0740(14)$ & $0.0941(17)$ & $0.0592(12)$ & $0.0181(13)$ & $0.0223(11)$ & $0.0103(12)$ \\
\hline $\mathrm{C} 5^{\prime}$ & $0.0915(16)$ & 0.0737 (14) & $0.0683(13)$ & $0.0026(12)$ & $0.0394(13)$ & $0.0054(11)$ \\
\hline C13 & $0.0696(13)$ & $0.0772(15)$ & $0.0733(14)$ & $-0.0081(12)$ & $0.0263(11)$ & $0.0079(12)$ \\
\hline
\end{tabular}


supporting information

\begin{tabular}{lllllll} 
C4 $^{\prime}$ & $0.0926(17)$ & $0.0731(15)$ & $0.0672(14)$ & $0.0018(13)$ & $0.0215(12)$ & $0.0134(12)$ \\
C12 & $0.0728(15)$ & $0.0937(19)$ & $0.0905(17)$ & $-0.0004(13)$ & $0.0351(13)$ & $0.0171(15)$ \\
C11 & $0.100(2)$ & $0.0925(19)$ & $0.0790(16)$ & $0.0190(16)$ & $0.0419(15)$ & $0.0129(14)$ \\
C3' & $0.0693(14)$ & $0.0779(15)$ & $0.0886(16)$ & $0.0102(12)$ & $0.0165(12)$ & $0.0210(13)$ \\
C10 & $0.100(2)$ & $0.0892(19)$ & $0.0812(17)$ & $0.0005(16)$ & $0.0299(15)$ & $-0.0058(14)$ \\
\hline
\end{tabular}

Geometric parameters $\left(\hat{A},{ }^{\circ}\right)$

\begin{tabular}{|c|c|c|c|}
\hline $\mathrm{O} 3-\mathrm{C} 7$ & $1.347(3)$ & $\mathrm{C} 1^{\prime}-\mathrm{C} 2^{\prime}$ & $1.384(3)$ \\
\hline $\mathrm{O} 3-\mathrm{C} 6$ & $1.441(3)$ & $\mathrm{C} 1-\mathrm{C} 2$ & $1.353(3)$ \\
\hline $\mathrm{O} 1^{\prime}-\mathrm{C} 7^{\prime}$ & $1.308(2)$ & $\mathrm{C} 8-\mathrm{C} 7$ & $1.476(3)$ \\
\hline $\mathrm{N} 1-\mathrm{C} 1$ & $1.383(2)$ & $\mathrm{C} 8-\mathrm{C} 9$ & $1.384(3)$ \\
\hline $\mathrm{N} 1-\mathrm{C} 3$ & $1.355(2)$ & $\mathrm{C} 8-\mathrm{C} 13$ & $1.386(3)$ \\
\hline $\mathrm{N} 1-\mathrm{C} 5$ & $1.466(3)$ & $\mathrm{C} 3-\mathrm{C} 4$ & $1.477(3)$ \\
\hline $\mathrm{O} 2^{\prime}-\mathrm{C} 7^{\prime}$ & $1.205(2)$ & $\mathrm{C} 6^{\prime}-\mathrm{C} 5^{\prime}$ & $1.383(3)$ \\
\hline N3-O1 & $1.230(2)$ & $\mathrm{C} 2^{\prime}-\mathrm{C} 3^{\prime}$ & $1.382(3)$ \\
\hline $\mathrm{N} 3-\mathrm{O} 2$ & $1.221(2)$ & $\mathrm{C} 5-\mathrm{C} 6$ & $1.504(3)$ \\
\hline $\mathrm{N} 3-\mathrm{C} 1$ & $1.422(3)$ & $\mathrm{C} 9-\mathrm{C} 10$ & $1.374(4)$ \\
\hline $\mathrm{N} 2-\mathrm{C} 3$ & $1.327(3)$ & $\mathrm{C} 5^{\prime}-\mathrm{C} 4^{\prime}$ & $1.376(3)$ \\
\hline $\mathrm{N} 2-\mathrm{C} 2$ & $1.360(3)$ & $\mathrm{C} 13-\mathrm{C} 12$ & $1.385(4)$ \\
\hline $\mathrm{O} 4-\mathrm{C} 7$ & $1.201(2)$ & $\mathrm{C} 4^{\prime}-\mathrm{C} 3^{\prime}$ & $1.379(4)$ \\
\hline $\mathrm{C} 1^{\prime}-\mathrm{C} 7^{\prime}$ & $1.488(3)$ & $\mathrm{C} 12-\mathrm{C} 11$ & $1.373(4)$ \\
\hline $\mathrm{C} 1^{\prime}-\mathrm{C} 6^{\prime}$ & $1.387(3)$ & $\mathrm{C} 11-\mathrm{C} 10$ & $1.370(4)$ \\
\hline $\mathrm{C} 7-\mathrm{O} 3-\mathrm{C} 6$ & $116.51(17)$ & $\mathrm{N} 1-\mathrm{C} 3-\mathrm{C} 4$ & $124.6(2)$ \\
\hline $\mathrm{C} 1-\mathrm{N} 1-\mathrm{C} 5$ & $129.89(17)$ & $\mathrm{N} 2-\mathrm{C} 3-\mathrm{N} 1$ & $111.14(17)$ \\
\hline $\mathrm{C} 3-\mathrm{N} 1-\mathrm{C} 1$ & $105.40(16)$ & $\mathrm{N} 2-\mathrm{C} 3-\mathrm{C} 4$ & $124.22(19)$ \\
\hline $\mathrm{C} 3-\mathrm{N} 1-\mathrm{C} 5$ & $124.57(18)$ & $\mathrm{C} 5^{\prime}-\mathrm{C} 6^{\prime}-\mathrm{C} 1^{\prime}$ & $120.1(2)$ \\
\hline $\mathrm{O} 1-\mathrm{N} 3-\mathrm{C} 1$ & $116.53(19)$ & $\mathrm{C} 3^{\prime}-\mathrm{C} 2^{\prime}-\mathrm{C} 1^{\prime}$ & $119.9(2)$ \\
\hline $\mathrm{O} 2-\mathrm{N} 3-\mathrm{O} 1$ & $123.4(2)$ & $\mathrm{O} 3-\mathrm{C} 7-\mathrm{C} 8$ & $112.86(17)$ \\
\hline $\mathrm{O} 2-\mathrm{N} 3-\mathrm{C} 1$ & $120.10(19)$ & $\mathrm{O} 4-\mathrm{C} 7-\mathrm{O} 3$ & $121.9(2)$ \\
\hline $\mathrm{C} 3-\mathrm{N} 2-\mathrm{C} 2$ & $106.95(17)$ & $\mathrm{O} 4-\mathrm{C} 7-\mathrm{C} 8$ & $125.2(2)$ \\
\hline $\mathrm{C} 6^{\prime}-\mathrm{C} 1^{\prime}-\mathrm{C} 7^{\prime}$ & $119.43(18)$ & $\mathrm{C} 1-\mathrm{C} 2-\mathrm{N} 2$ & $108.58(19)$ \\
\hline $\mathrm{C} 2^{\prime}-\mathrm{C} 1^{\prime}-\mathrm{C} 7^{\prime}$ & $120.86(18)$ & $\mathrm{N} 1-\mathrm{C} 5-\mathrm{C} 6$ & $111.62(19)$ \\
\hline $\mathrm{C} 2^{\prime}-\mathrm{C} 1^{\prime}-\mathrm{C} 6^{\prime}$ & $119.65(19)$ & $\mathrm{C} 10-\mathrm{C} 9-\mathrm{C} 8$ & $120.7(2)$ \\
\hline $\mathrm{N} 1-\mathrm{C} 1-\mathrm{N} 3$ & $125.81(18)$ & $\mathrm{O} 3-\mathrm{C} 6-\mathrm{C} 5$ & $107.62(19)$ \\
\hline $\mathrm{C} 2-\mathrm{C} 1-\mathrm{N} 1$ & $107.93(18)$ & $\mathrm{C} 4^{\prime}-\mathrm{C} 5^{\prime}-\mathrm{C} 6^{\prime}$ & $119.9(2)$ \\
\hline $\mathrm{C} 2-\mathrm{C} 1-\mathrm{N} 3$ & $126.15(19)$ & $\mathrm{C} 12-\mathrm{C} 13-\mathrm{C} 8$ & $119.5(3)$ \\
\hline $\mathrm{C} 9-\mathrm{C} 8-\mathrm{C} 7$ & $117.7(2)$ & $\mathrm{C} 5^{\prime}-\mathrm{C} 4^{\prime}-\mathrm{C} 3^{\prime}$ & $120.2(2)$ \\
\hline $\mathrm{C} 9-\mathrm{C} 8-\mathrm{C} 13$ & $119.5(2)$ & $\mathrm{C} 11-\mathrm{C} 12-\mathrm{C} 13$ & $120.1(3)$ \\
\hline $\mathrm{C} 13-\mathrm{C} 8-\mathrm{C} 7$ & $122.9(2)$ & $\mathrm{C} 10-\mathrm{C} 11-\mathrm{C} 12$ & $120.6(3)$ \\
\hline $\mathrm{O} 1^{\prime}-\mathrm{C}^{\prime}-\mathrm{C} 1^{\prime}$ & $113.23(17)$ & $\mathrm{C} 4^{\prime}-\mathrm{C} 3^{\prime}-\mathrm{C} 2^{\prime}$ & $120.2(2)$ \\
\hline $\mathrm{O} 2^{\prime}-\mathrm{C}^{\prime}-\mathrm{O}^{\prime}$ & $123.35(19)$ & $\mathrm{C} 11-\mathrm{C} 10-\mathrm{C} 9$ & $119.6(3)$ \\
\hline $\mathrm{O} 2^{\prime}-\mathrm{C}^{\prime}-\mathrm{C}^{\prime}$ & $123.38(19)$ & & \\
\hline
\end{tabular}




\section{2-(2-Methyl-5-nitro-1H-imidazol-1-yl)ethyl benzoate- $\beta$-naphthol (1/1) (bzmdnaf)}

Crystal data

$$
\begin{aligned}
& \mathrm{C}_{13} \mathrm{H}_{13} \mathrm{~N}_{3} \mathrm{O}_{4} \cdot \mathrm{C}_{10} \mathrm{H}_{8} \mathrm{O} \\
& M_{r}=419.43 \\
& \text { Monoclinic, } P 2_{1} / c \\
& a=8.5105(8) \AA \\
& b=22.387(2) \AA \\
& c=11.7752(11) \AA \\
& \beta=108.664(4)^{\circ} \\
& V=2125.4(3) \AA^{3} \\
& Z=4
\end{aligned}
$$

\section{Data collection}

Bruker D8 VENTURE Kappa Duo PHOTON II
CPAD
diffractometer
$\varphi$ and $\omega$ scans
Absorption correction: multi-scan
(SADABS; Bruker, 2016)

$T_{\min }=0.678, T_{\max }=1.000$

39131 measured reflections

\section{Refinement}

Refinement on $F^{2}$

Least-squares matrix: full

$R\left[F^{2}>2 \sigma\left(F^{2}\right)\right]=0.058$

$w R\left(F^{2}\right)=0.193$

$S=1.06$

3884 reflections

365 parameters

0 restraints

Hydrogen site location: difference Fourier map

All $\mathrm{H}$-atom parameters refined

$$
\begin{aligned}
& F(000)=880 \\
& D_{\mathrm{x}}=1.311 \mathrm{Mg} \mathrm{m}^{-3} \\
& \text { Cu } K \alpha \text { radiation, } \lambda=1.54178 \AA \\
& \text { Cell parameters from } 9803 \text { reflections } \\
& \theta=4.4-69.6^{\circ} \\
& \mu=0.78 \mathrm{~mm}^{-1} \\
& T=300 \mathrm{~K} \\
& \text { Prism, clear light yellow } \\
& 0.42 \times 0.09 \times 0.07 \mathrm{~mm}
\end{aligned}
$$

3884 independent reflections

2810 reflections with $I>2 \sigma(I)$

$R_{\text {int }}=0.084$

$\theta_{\max }=68.3^{\circ}, \theta_{\min }=4.0^{\circ}$

$h=-10 \rightarrow 10$

$k=-26 \rightarrow 26$

$l=-14 \rightarrow 13$

\section{Special details}

Geometry. All esds (except the esd in the dihedral angle between two 1.s. planes) are estimated using the full covariance matrix. The cell esds are taken into account individually in the estimation of esds in distances, angles and torsion angles; correlations between esds in cell parameters are only used when they are defined by crystal symmetry. An approximate (isotropic) treatment of cell esds is used for estimating esds involving l.s. planes.

Refinement. Single-crystal X-ray diffraction data collection ( scans and $\omega$ scans with $\kappa$ and $\theta$ offsets) were performed at room temperature on a Bruker AXS D8 VENTURE equipped with a Kappa goniometer, PHOTON II CPAD detector, a Mo $K \alpha$ INCOATEC I $\mu$ S 3.0 microfocus source $(\lambda=0.71073 \AA)$. The crystal centring, unit-cell determination, refinement of the unit-cell parameters and data collection was controlled through the program APEX3 (Bruker, 2012). The frame integration was performed using SAINT (Bruker, 2016) and the intensities were scaled and absorption corrected using SADABS (Bruker, 2001). Using OLEX2 (Dolomanov et al., 2009), the structure was solved by intrinsic phasing using SHELXT (Sheldrick, 2015) and refined by full-matrix least-squares calculation based on $F^{2}$ for all reflection using SHELXL (Sheldrick, 2007). All non-H atoms were refined anisotropically.

Fractional atomic coordinates and isotropic or equivalent isotropic displacement parameters $\left(\AA^{2}\right)$

\begin{tabular}{lllll}
\hline & $x$ & $y$ & $z$ & $U_{\text {iso }} * / U_{\text {eq }}$ \\
\hline O3 & $-0.36072(19)$ & $0.49428(7)$ & $0.19944(13)$ & $0.0624(4)$
\end{tabular}




\begin{tabular}{|c|c|c|c|c|}
\hline $\mathrm{O} 2$ & -0.2808 & $0.51266(9)$ & $0.57654(16)$ & $0.0842(6)$ \\
\hline N1 & $-0.2220(2)$ & $0.58230(8)$ & $0.39335(15)$ & $0.0563(4)$ \\
\hline $\mathrm{O} 1$ & $-0.0234(3)$ & $0.52273(9)$ & $0.68439(16)$ & $0.0850(6)$ \\
\hline $\mathrm{O} 1^{\prime}$ & $0.2411(3)$ & $0.70227(10)$ & $0.35022(19)$ & $0.0908(6)$ \\
\hline N3 & $-0.1417(3)$ & $0.53251(9)$ & $0.59314(17)$ & $0.0652(5)$ \\
\hline $\mathrm{O} 4$ & $-0.3769(3)$ & $0.39564(9)$ & $0.21856(18)$ & $0.0892(6)$ \\
\hline N2 & $0.0181(2)$ & $0.62642(9)$ & $0.40900(18)$ & $0.0681(5)$ \\
\hline $\mathrm{C} 1$ & $-0.1092(3)$ & $0.56838(9)$ & $0.50416(18)$ & $0.0562(5)$ \\
\hline C3 & -0.1374 & $0.61722(10)$ & $0.3393(2)$ & $0.0634(6)$ \\
\hline $\mathrm{C} 8$ & $-0.2958(3)$ & $0.43345(12)$ & 0.05661 (19) & $0.0658(6)$ \\
\hline $\mathrm{C} 2$ & $0.0348(3)$ & 0.59617 (11) & $0.5115(2)$ & $0.0649(6)$ \\
\hline $\mathrm{C} 7$ & $-0.3486(3)$ & 0.43791 (11) & $0.1652(2)$ & $0.0633(6)$ \\
\hline $\mathrm{C} 10^{\prime}$ & $0.4667(3)$ & $0.67583(11)$ & $0.5266(2)$ & $0.0675(6)$ \\
\hline $\mathrm{C} 5$ & $-0.3963(3)$ & $0.56412(11)$ & $0.3424(2)$ & $0.0645(6)$ \\
\hline $\mathrm{C} 9^{\prime}$ & $0.6381(3)$ & $0.67996(10)$ & $0.5925(2)$ & $0.0688(6)$ \\
\hline $\mathrm{C} 1^{\prime}$ & $0.4025(3)$ & $0.70428(11)$ & $0.4195(2)$ & $0.0727(7)$ \\
\hline C6 & $-0.4166(3)$ & $0.50046(11)$ & $0.3028(2)$ & $0.0623(6)$ \\
\hline $\mathrm{C} 9$ & $-0.2418(3)$ & $0.48176(15)$ & $0.0078(2)$ & $0.0772(7)$ \\
\hline $\mathrm{C} 4^{\prime}$ & $0.7415(3)$ & $0.71446(11)$ & $0.5461(3)$ & $0.0799(8)$ \\
\hline $\mathrm{C} 4$ & $-0.2096(5)$ & $0.64272(17)$ & $0.2172(3)$ & $0.0859(8)$ \\
\hline $\mathrm{C} 13$ & $-0.2986(4)$ & $0.37744(15)$ & 0.0059 (2) & $0.0836(8)$ \\
\hline $\mathrm{C} 8^{\prime}$ & $0.7088(4)$ & $0.65030(14)$ & $0.7035(3)$ & $0.0846(8)$ \\
\hline $\mathrm{C} 2^{\prime}$ & $0.5090(5)$ & 0.73895 (14) & $0.3747(4)$ & $0.0921(9)$ \\
\hline $\mathrm{C} 3^{\prime}$ & $0.6720(4)$ & 0.74335 (14) & $0.4367(4)$ & 0.0989 (11) \\
\hline $\mathrm{C} 12$ & $-0.2455(5)$ & $0.37083(19)$ & -0.0939 (3) & $0.0984(10)$ \\
\hline C11 & $-0.1911(4)$ & $0.4196(2)$ & $-0.1412(3)$ & $0.0977(10)$ \\
\hline $\mathrm{C} 5^{\prime}$ & $0.9152(4)$ & $0.71857(15)$ & $0.6162(5)$ & $0.1008(12)$ \\
\hline $\mathrm{C} 10$ & $-0.1889(4)$ & $0.47476(19)$ & $-0.0920(3)$ & $0.0913(9)$ \\
\hline $\mathrm{C}^{\prime}$ & $0.9762(5)$ & $0.68936(17)$ & $0.7222(4)$ & $0.1071(12)$ \\
\hline $\mathrm{C} 7^{\prime}$ & $0.8736(4)$ & $0.65473(17)$ & $0.7664(3)$ & $0.0969(10)$ \\
\hline $\mathrm{H} 5 \mathrm{~A}$ & $-0.448(4)$ & $0.5694(12)$ & $0.403(3)$ & $0.082(8)^{*}$ \\
\hline H10' & $0.394(3)$ & $0.6541(11)$ & $0.559(2)$ & $0.070(7)^{*}$ \\
\hline H6A & -0.540 & $0.4903(11)$ & $0.280(2)$ & $0.076(7)^{*}$ \\
\hline H5B & $-0.448(3)$ & $0.5927(11)$ & $0.277(2)$ & $0.071(7)^{*}$ \\
\hline H6B & $-0.355(3)$ & $0.4734(12)$ & $0.365(2)$ & $0.071(7)^{*}$ \\
\hline $\mathrm{H} 8^{\prime}$ & $0.629(5)$ & $0.6273(16)$ & $0.736(3)$ & $0.125(12)^{*}$ \\
\hline H10 & $-0.146(4)$ & $0.5098(15)$ & $-0.128(3)$ & $0.112(11)^{*}$ \\
\hline H13 & $-0.341(4)$ & $0.3442(15)$ & $0.041(3)$ & $0.096(10)^{*}$ \\
\hline H9 & $-0.246(4)$ & $0.5203(15)$ & $0.043(3)$ & $0.095(10)^{*}$ \\
\hline $\mathrm{H} 1^{\prime}$ & $0.180(4)$ & $0.6777(16)$ & $0.382(3)$ & $0.107(11)^{*}$ \\
\hline $\mathrm{H} 2$ & $0.134(4)$ & $0.5959(12)$ & $0.575(3)$ & $0.083(8)^{*}$ \\
\hline $\mathrm{H} 4 \mathrm{~A}$ & $-0.132(5)$ & $0.6636(18)$ & $0.200(3)$ & $0.122(13)^{*}$ \\
\hline H3' & $0.751(4)$ & $0.7664(16)$ & $0.413(3)$ & $0.113(10)^{*}$ \\
\hline $\mathrm{H}^{\prime}$ & $1.104(5)$ & $0.6919(18)$ & $0.771(3)$ & $0.133(13)^{*}$ \\
\hline H12 & $-0.249(5)$ & $0.3291(18)$ & $-0.124(3)$ & $0.124(12)^{*}$ \\
\hline H4B & $-0.267(5)$ & $0.6107(19)$ & $0.157(4)$ & $0.138(14)^{*}$ \\
\hline H11 & -0.150 & $0.4097(16)$ & -0.213 & $0.120(11)^{*}$ \\
\hline $\mathrm{H} 2^{\prime}$ & 0.458 (4) & $0.7552(16)$ & $0.306(3)$ & $0.102(11)^{*}$ \\
\hline
\end{tabular}




\begin{tabular}{lllll}
$\mathrm{H} 4 \mathrm{C}$ & $-0.304(6)$ & $0.670(2)$ & $0.212(4)$ & $0.148(15)^{*}$ \\
$\mathrm{H} 5^{\prime}$ & $0.974(4)$ & $0.7413(15)$ & $0.573(3)$ & $0.104(10)^{*}$ \\
$\mathrm{H} 7^{\prime}$ & $0.937(6)$ & $0.634(2)$ & $0.855(5)$ & $0.170(18)^{*}$ \\
\hline
\end{tabular}

Atomic displacement parameters $\left(\AA^{2}\right)$

\begin{tabular}{|c|c|c|c|c|c|c|}
\hline & $U^{11}$ & $U^{22}$ & $U^{33}$ & $U^{12}$ & $U^{13}$ & $U^{23}$ \\
\hline $\mathrm{O} 3$ & $0.0638(9)$ & $0.0724(10)$ & $0.0572(8)$ & $-0.0028(7)$ & $0.0280(7)$ & 0.0008 (7) \\
\hline $\mathrm{O} 2$ & $0.0876(13)$ & $0.1041(14)$ & $0.0733(11)$ & $-0.0111(10)$ & $0.0431(10)$ & 0.0035 (9) \\
\hline N1 & $0.0562(9)$ & $0.0573(10)$ & $0.0581(10)$ & $0.0053(8)$ & $0.0221(8)$ & $0.0002(8)$ \\
\hline O1 & $0.0934(13)$ & $0.0978(14)$ & $0.0590(10)$ & $0.0078(10)$ & $0.0177(9)$ & $0.0115(9)$ \\
\hline $\mathrm{O} 1^{\prime}$ & $0.0843(13)$ & $0.0958(14)$ & $0.0938(13)$ & $0.0026(10)$ & $0.0305(11)$ & $0.0253(11)$ \\
\hline N3 & $0.0746(13)$ & $0.0691(12)$ & $0.0586(11)$ & $0.0051(10)$ & $0.0308(10)$ & $-0.0026(9)$ \\
\hline $\mathrm{O} 4$ & $0.1245(16)$ & $0.0762(11)$ & $0.0852(12)$ & $-0.0154(10)$ & $0.0589(11)$ & $-0.0029(9)$ \\
\hline N2 & $0.0683(12)$ & $0.0661(12)$ & $0.0741(12)$ & $-0.0026(9)$ & $0.0287(10)$ & $0.0014(9)$ \\
\hline $\mathrm{C} 1$ & $0.0613(12)$ & $0.0563(11)$ & $0.0538(11)$ & $0.0060(9)$ & $0.0226(9)$ & $0.0004(9)$ \\
\hline $\mathrm{C} 3$ & $0.0694(14)$ & $0.0577(12)$ & 0.0677 (13) & $0.0034(10)$ & $0.0286(11)$ & $0.0043(10)$ \\
\hline $\mathrm{C} 8$ & $0.0571(12)$ & 0.0904 (17) & $0.0500(11)$ & 0.0015 (11) & $0.0173(9)$ & $-0.0006(10)$ \\
\hline $\mathrm{C} 2$ & $0.0649(14)$ & $0.0645(13)$ & $0.0660(14)$ & 0.0048 (11) & $0.0219(11)$ & $-0.0037(11)$ \\
\hline $\mathrm{C} 7$ & $0.0619(13)$ & $0.0732(15)$ & $0.0581(12)$ & $-0.0052(11)$ & $0.0239(10)$ & $-0.0017(10)$ \\
\hline $\mathrm{C} 10^{\prime}$ & 0.0707 (14) & $0.0613(13)$ & $0.0796(15)$ & $-0.0055(11)$ & $0.0368(12)$ & $-0.0040(11)$ \\
\hline C5 & $0.0541(12)$ & $0.0740(15)$ & $0.0679(14)$ & $0.0079(11)$ & $0.0230(11)$ & 0.0019 (11) \\
\hline $\mathrm{C} 9^{\prime}$ & $0.0758(15)$ & $0.0580(13)$ & $0.0816(16)$ & $-0.0034(11)$ & $0.0381(12)$ & $-0.0181(11)$ \\
\hline $\mathrm{C} 1^{\prime}$ & $0.0757(16)$ & 0.0597 (13) & $0.0914(17)$ & 0.0017 (11) & $0.0390(14)$ & $0.0034(12)$ \\
\hline $\mathrm{C} 6$ & $0.0593(13)$ & $0.0755(15)$ & $0.0585(13)$ & $-0.0031(11)$ & $0.0278(10)$ & $-0.0014(11)$ \\
\hline C9 & $0.0732(15)$ & $0.103(2)$ & $0.0595(14)$ & $-0.0112(14)$ & $0.0272(12)$ & $-0.0008(13)$ \\
\hline $\mathrm{C} 4^{\prime}$ & $0.0784(17)$ & $0.0543(13)$ & $0.121(2)$ & $-0.0065(12)$ & $0.0508(16)$ & $-0.0169(14)$ \\
\hline $\mathrm{C} 4$ & $0.094(2)$ & $0.088(2)$ & $0.0754(17)$ & $-0.0009(18)$ & $0.0263(16)$ & $0.0244(15)$ \\
\hline $\mathrm{C} 13$ & 0.0924 (19) & $0.094(2)$ & $0.0640(15)$ & $0.0123(16)$ & 0.0245 (14) & $-0.0047(14)$ \\
\hline $\mathrm{C} 8^{\prime}$ & $0.0856(18)$ & $0.0873(19)$ & $0.0835(18)$ & $-0.0045(15)$ & $0.0306(15)$ & $-0.0211(15)$ \\
\hline $\mathrm{C} 2^{\prime}$ & $0.106(2)$ & $0.0732(17)$ & $0.113(2)$ & $0.0034(16)$ & $0.057(2)$ & 0.0197 (17) \\
\hline $\mathrm{C} 3^{\prime}$ & $0.096(2)$ & $0.0680(17)$ & $0.157(3)$ & $-0.0086(15)$ & $0.075(2)$ & $0.0096(18)$ \\
\hline $\mathrm{C} 12$ & $0.103(2)$ & $0.123(3)$ & $0.0663(16)$ & $0.030(2)$ & $0.0229(16)$ & $-0.0136(17)$ \\
\hline $\mathrm{C} 11$ & $0.0785(18)$ & $0.157(3)$ & $0.0607(16)$ & $0.0163(19)$ & $0.0268(13)$ & 0.0002 (19) \\
\hline $\mathrm{C} 5^{\prime}$ & $0.083(2)$ & $0.0666(17)$ & $0.168(4)$ & $-0.0161(15)$ & $0.062(2)$ & $-0.032(2)$ \\
\hline $\mathrm{C} 10$ & $0.0742(17)$ & $0.140(3)$ & $0.0655(16)$ & $-0.0110(17)$ & $0.0302(13)$ & $0.0037(18)$ \\
\hline$C 6^{\prime}$ & $0.085(2)$ & $0.088(2)$ & $0.138(3)$ & $-0.0008(18)$ & $0.021(2)$ & $-0.042(2)$ \\
\hline$C 7^{\prime}$ & 0.0807 (19) & $0.100(2)$ & 0.104 (2) & $-0.0034(17)$ & $0.0213(17)$ & -0.0365 (19) \\
\hline
\end{tabular}

Geometric parameters $\left(\AA,{ }^{o}\right)$

\begin{tabular}{llll}
\hline $\mathrm{O} 3-\mathrm{C} 7$ & $1.339(3)$ & $\mathrm{C} 1^{\prime}-\mathrm{C} 2^{\prime}$ & $1.417(4)$ \\
$\mathrm{O} 3-\mathrm{C} 6$ & $1.448(3)$ & $\mathrm{C} 6-\mathrm{H} 6 \mathrm{~A}$ & $1.02(3)$ \\
$\mathrm{O} 2-\mathrm{N} 3$ & $1.221(3)$ & $\mathrm{C} 6-\mathrm{H} 6 \mathrm{~B}$ & $0.96(3)$ \\
$\mathrm{N} 1-\mathrm{C} 1$ & $1.385(3)$ & $\mathrm{C} 9-\mathrm{C} 10$ & $1.395(4)$ \\
$\mathrm{N} 1-\mathrm{C} 3$ & $1.352(3)$ & $\mathrm{C} 9-\mathrm{H} 9$ & $0.96(3)$ \\
$\mathrm{N} 1-\mathrm{C} 5$ & $1.468(3)$ & $\mathrm{C} 4^{\prime}-\mathrm{C} 3^{\prime}$ & $1.393(5)$ \\
$\mathrm{O} 1-\mathrm{N} 3$ & $1.234(3)$ & $\mathrm{C} 4^{\prime}-\mathrm{C} 5^{\prime}$ & $1.447(5)$
\end{tabular}




\begin{tabular}{|c|c|c|c|}
\hline $\mathrm{O} 1^{\prime}-\mathrm{C}^{\prime}$ & $1.356(3)$ & $\mathrm{C} 4-\mathrm{H} 4 \mathrm{~A}$ & $0.88(4)$ \\
\hline $\mathrm{O} 1^{\prime}-\mathrm{H} 1^{\prime}$ & $0.91(4)$ & $\mathrm{C} 4-\mathrm{H} 4 \mathrm{~B}$ & $1.02(5)$ \\
\hline $\mathrm{N} 3-\mathrm{C} 1$ & $1.416(3)$ & $\mathrm{C} 4-\mathrm{H} 4 \mathrm{C}$ & $1.00(5)$ \\
\hline $\mathrm{O} 4-\mathrm{C} 7$ & $1.202(3)$ & $\mathrm{C} 13-\mathrm{C} 12$ & $1.395(4)$ \\
\hline $\mathrm{N} 2-\mathrm{C} 3$ & $1.332(3)$ & $\mathrm{C} 13-\mathrm{H} 13$ & $0.98(3)$ \\
\hline $\mathrm{N} 2-\mathrm{C} 2$ & $1.351(3)$ & $\mathrm{C} 8^{\prime}-\mathrm{C} 7^{\prime}$ & $1.364(4)$ \\
\hline $\mathrm{C} 1-\mathrm{C} 2$ & $1.352(3)$ & $\mathrm{C} 8^{\prime}-\mathrm{H} 8^{\prime}$ & $1.02(4)$ \\
\hline $\mathrm{C} 3-\mathrm{C} 4$ & $1.484(4)$ & $\mathrm{C} 2^{\prime}-\mathrm{C} 3^{\prime}$ & $1.348(5)$ \\
\hline $\mathrm{C} 8-\mathrm{C} 7$ & $1.488(3)$ & $\mathrm{C} 2^{\prime}-\mathrm{H} 2^{\prime}$ & $0.87(3)$ \\
\hline $\mathrm{C} 8-\mathrm{C} 9$ & $1.371(4)$ & $\mathrm{C} 3^{\prime}-\mathrm{H} 3^{\prime}$ & $0.95(4)$ \\
\hline $\mathrm{C} 8-\mathrm{C} 13$ & $1.386(4)$ & $\mathrm{C} 12-\mathrm{C} 11$ & $1.372(5)$ \\
\hline $\mathrm{C} 2-\mathrm{H} 2$ & $0.93(3)$ & $\mathrm{C} 12-\mathrm{H} 12$ & $1.00(4)$ \\
\hline $\mathrm{C} 10^{\prime}-\mathrm{C} 9^{\prime}$ & $1.419(4)$ & $\mathrm{C} 11-\mathrm{C} 10$ & $1.362(5)$ \\
\hline $\mathrm{C} 10^{\prime}-\mathrm{C} 1^{\prime}$ & $1.361(4)$ & C11-H11 & $1.04(4)$ \\
\hline $\mathrm{C} 10^{\prime}-\mathrm{H} 10^{\prime}$ & $0.95(3)$ & $\mathrm{C} 5^{\prime}-\mathrm{C} 6^{\prime}$ & $1.356(6)$ \\
\hline $\mathrm{C} 5-\mathrm{C} 6$ & $1.492(3)$ & $\mathrm{C} 5^{\prime}-\mathrm{H} 5^{\prime}$ & $0.96(3)$ \\
\hline $\mathrm{C} 5-\mathrm{H} 5 \mathrm{~A}$ & $0.95(3)$ & $\mathrm{C} 10-\mathrm{H} 10$ & $1.01(4)$ \\
\hline C5-H5B & $0.99(3)$ & $\mathrm{C} 6^{\prime}-\mathrm{C} 7^{\prime}$ & $1.388(6)$ \\
\hline $\mathrm{C} 9^{\prime}-\mathrm{C} 4^{\prime}$ & $1.406(4)$ & $\mathrm{C} 6^{\prime}-\mathrm{H} 6^{\prime}$ & $1.05(4)$ \\
\hline $\mathrm{C} 9^{\prime}-\mathrm{C} 8^{\prime}$ & $1.418(4)$ & $\mathrm{C} 7^{\prime}-\mathrm{H} 7^{\prime}$ & $1.11(5)$ \\
\hline $\mathrm{C} 7-\mathrm{O} 3-\mathrm{C} 6$ & $114.89(17)$ & $\mathrm{H} 6 \mathrm{~A}-\mathrm{C} 6-\mathrm{H} 6 \mathrm{~B}$ & $109(2)$ \\
\hline $\mathrm{C} 1-\mathrm{N} 1-\mathrm{C} 5$ & $128.84(19)$ & $\mathrm{C} 8-\mathrm{C} 9-\mathrm{C} 10$ & $120.4(3)$ \\
\hline $\mathrm{C} 3-\mathrm{N} 1-\mathrm{C} 1$ & $105.12(18)$ & $\mathrm{C} 8-\mathrm{C} 9-\mathrm{H} 9$ & $117.6(19)$ \\
\hline $\mathrm{C} 3-\mathrm{N} 1-\mathrm{C} 5$ & $126.04(19)$ & $\mathrm{C} 10-\mathrm{C} 9-\mathrm{H} 9$ & $122(2)$ \\
\hline $\mathrm{C} 1^{\prime}-\mathrm{O} 1^{\prime}-\mathrm{H}^{\prime}$ & $112(2)$ & $\mathrm{C} 9^{\prime}-\mathrm{C} 4^{\prime}-\mathrm{C} 5^{\prime}$ & $117.7(3)$ \\
\hline $\mathrm{O} 2-\mathrm{N} 3-\mathrm{O} 1$ & $123.6(2)$ & $\mathrm{C} 3^{\prime}-\mathrm{C} 4^{\prime}-\mathrm{C} 9^{\prime}$ & $118.7(3)$ \\
\hline $\mathrm{O} 2-\mathrm{N} 3-\mathrm{C} 1$ & $119.8(2)$ & $\mathrm{C} 3^{\prime}-\mathrm{C} 4^{\prime}-\mathrm{C} 5^{\prime}$ & $123.5(3)$ \\
\hline $\mathrm{O} 1-\mathrm{N} 3-\mathrm{C} 1$ & $116.5(2)$ & $\mathrm{C} 3-\mathrm{C} 4-\mathrm{H} 4 \mathrm{~A}$ & $109(3)$ \\
\hline $\mathrm{C} 3-\mathrm{N} 2-\mathrm{C} 2$ & $105.8(2)$ & $\mathrm{C} 3-\mathrm{C} 4-\mathrm{H} 4 \mathrm{~B}$ & $111(2)$ \\
\hline $\mathrm{N} 1-\mathrm{C} 1-\mathrm{N} 3$ & $125.74(19)$ & $\mathrm{C} 3-\mathrm{C} 4-\mathrm{H} 4 \mathrm{C}$ & $112(3)$ \\
\hline $\mathrm{C} 2-\mathrm{C} 1-\mathrm{N} 1$ & $107.28(19)$ & $\mathrm{H} 4 \mathrm{~A}-\mathrm{C} 4-\mathrm{H} 4 \mathrm{~B}$ & $116(3)$ \\
\hline $\mathrm{C} 2-\mathrm{C} 1-\mathrm{N} 3$ & $127.0(2)$ & $\mathrm{H} 4 \mathrm{~A}-\mathrm{C} 4-\mathrm{H} 4 \mathrm{C}$ & $107(4)$ \\
\hline $\mathrm{N} 1-\mathrm{C} 3-\mathrm{C} 4$ & $124.1(2)$ & $\mathrm{H} 4 \mathrm{~B}-\mathrm{C} 4-\mathrm{H} 4 \mathrm{C}$ & $101(4)$ \\
\hline $\mathrm{N} 2-\mathrm{C} 3-\mathrm{N} 1$ & $111.9(2)$ & $\mathrm{C} 8-\mathrm{C} 13-\mathrm{C} 12$ & $119.5(3)$ \\
\hline $\mathrm{N} 2-\mathrm{C} 3-\mathrm{C} 4$ & $124.0(2)$ & $\mathrm{C} 8-\mathrm{C} 13-\mathrm{H} 13$ & $117.7(19)$ \\
\hline $\mathrm{C} 9-\mathrm{C} 8-\mathrm{C} 7$ & $122.8(2)$ & $\mathrm{C} 12-\mathrm{C} 13-\mathrm{H} 13$ & $122.7(19)$ \\
\hline $\mathrm{C} 9-\mathrm{C} 8-\mathrm{C} 13$ & $119.7(2)$ & $\mathrm{C} 9^{\prime}-\mathrm{C} 8^{\prime}-\mathrm{H} 8^{\prime}$ & $116(2)$ \\
\hline $\mathrm{C} 13-\mathrm{C} 8-\mathrm{C} 7$ & $117.5(2)$ & $\mathrm{C} 7^{\prime}-\mathrm{C} 8^{\prime}-\mathrm{C} 9^{\prime}$ & $121.7(3)$ \\
\hline $\mathrm{N} 2-\mathrm{C} 2-\mathrm{C} 1$ & $109.9(2)$ & $\mathrm{C} 7^{\prime}-\mathrm{C} 8^{\prime}-\mathrm{H} 8^{\prime}$ & $122(2)$ \\
\hline $\mathrm{N} 2-\mathrm{C} 2-\mathrm{H} 2$ & $121.8(18)$ & $\mathrm{C} 1^{\prime}-\mathrm{C} 2^{\prime}-\mathrm{H} 2^{\prime}$ & $113(2)$ \\
\hline $\mathrm{C} 1-\mathrm{C} 2-\mathrm{H} 2$ & $128.3(18)$ & $\mathrm{C} 3^{\prime}-\mathrm{C} 2^{\prime}-\mathrm{C} 1^{\prime}$ & $120.5(3)$ \\
\hline $\mathrm{O} 3-\mathrm{C} 7-\mathrm{C} 8$ & $113.3(2)$ & $\mathrm{C} 3^{\prime}-\mathrm{C} 2^{\prime}-\mathrm{H} 2^{\prime}$ & $126(2)$ \\
\hline $\mathrm{O} 4-\mathrm{C} 7-\mathrm{O} 3$ & $122.5(2)$ & $\mathrm{C} 4^{\prime}-\mathrm{C} 3^{\prime}-\mathrm{H} 3^{\prime}$ & $113(2)$ \\
\hline $\mathrm{O} 4-\mathrm{C} 7-\mathrm{C} 8$ & $124.2(2)$ & $\mathrm{C} 2^{\prime}-\mathrm{C} 3^{\prime}-\mathrm{C} 4^{\prime}$ & $121.7(3)$ \\
\hline $\mathrm{C}^{\prime}-\mathrm{C} 10^{\prime}-\mathrm{H} 10^{\prime}$ & $119.9(15)$ & $\mathrm{C} 2^{\prime}-\mathrm{C} 3^{\prime}-\mathrm{H} 3^{\prime}$ & $125(2)$ \\
\hline $\mathrm{C} 1^{\prime}-\mathrm{C} 10^{\prime}-\mathrm{C} 9^{\prime}$ & $120.9(2)$ & $\mathrm{C} 13-\mathrm{C} 12-\mathrm{H} 12$ & $115(2)$ \\
\hline $\mathrm{C} 1^{\prime}-\mathrm{C} 10^{\prime}-\mathrm{H} 10^{\prime}$ & $119.1(15)$ & $\mathrm{C} 11-\mathrm{C} 12-\mathrm{C} 13$ & $120.0(3)$ \\
\hline
\end{tabular}




\begin{tabular}{llll}
$\mathrm{N} 1-\mathrm{C} 5-\mathrm{C} 6$ & $113.15(19)$ & $\mathrm{C} 11-\mathrm{C} 12-\mathrm{H} 12$ & $125(2)$ \\
$\mathrm{N} 1-\mathrm{C} 5-\mathrm{H} 5 \mathrm{~A}$ & $107.8(17)$ & $\mathrm{C} 12-\mathrm{C} 11-\mathrm{H} 11$ & $114(2)$ \\
$\mathrm{N} 1-\mathrm{C} 5-\mathrm{H} 5 \mathrm{~B}$ & $106.3(14)$ & $\mathrm{C} 10-\mathrm{C} 11-\mathrm{C} 12$ & $120.6(3)$ \\
$\mathrm{C} 6-\mathrm{C} 5-\mathrm{H} 5 \mathrm{~A}$ & $108.5(17)$ & $\mathrm{C} 10-\mathrm{C} 11-\mathrm{H} 11$ & $125(2)$ \\
$\mathrm{C} 6-\mathrm{C} 5-\mathrm{H} 5 \mathrm{~B}$ & $113.1(14)$ & $\mathrm{C} 4^{\prime}-\mathrm{C} 5^{\prime}-\mathrm{H} 5^{\prime}$ & $110(2)$ \\
$\mathrm{H} 5 \mathrm{~A}-\mathrm{C} 5-\mathrm{H} 5 \mathrm{~B}$ & $108(2)$ & $\mathrm{C} 6^{\prime}-\mathrm{C} 5^{\prime}-\mathrm{C} 4^{\prime}$ & $121.0(3)$ \\
$\mathrm{C} 4^{\prime}-\mathrm{C} 9^{\prime}-\mathrm{C} 10^{\prime}$ & $119.0(3)$ & $\mathrm{C} 6^{\prime}-\mathrm{C} 5^{\prime}-\mathrm{H} 5^{\prime}$ & $129(2)$ \\
$\mathrm{C} 4^{\prime}-\mathrm{C} 9^{\prime}-\mathrm{C} 8^{\prime}$ & $118.8(3)$ & $\mathrm{C} 9-\mathrm{C} 10-\mathrm{H} 10$ & $121(2)$ \\
$\mathrm{C} 8^{\prime}-\mathrm{C} 9^{\prime}-\mathrm{C} 10^{\prime}$ & $122.2(2)$ & $\mathrm{C} 11-\mathrm{C} 10-\mathrm{C} 9$ & $119.8(3)$ \\
$\mathrm{O} 1^{\prime}-\mathrm{C} 1^{\prime}-\mathrm{C} 10^{\prime}$ & $124.8(2)$ & $\mathrm{C} 11-\mathrm{C} 10-\mathrm{H} 10$ & $119(2)$ \\
$\mathrm{O} 1^{\prime}-\mathrm{C} 1^{\prime}-\mathrm{C} 2^{\prime}$ & $116.1(3)$ & $\mathrm{C} 5^{\prime}-\mathrm{C} 6^{\prime}-\mathrm{C} 7^{\prime}$ & $120.9(4)$ \\
$\mathrm{C} 10^{\prime}-\mathrm{C} 1^{\prime}-\mathrm{C} 2^{\prime}$ & $119.1(3)$ & $\mathrm{C} 5^{\prime}-\mathrm{C} 6^{\prime}-\mathrm{H} 6^{\prime}$ & $120(2)$ \\
$\mathrm{O} 3-\mathrm{C} 6-\mathrm{C} 5$ & $108.53(19)$ & $\mathrm{C} 7^{\prime}-\mathrm{C} 6^{\prime}-\mathrm{H} 6^{\prime}$ & $119(2)$ \\
$\mathrm{O} 3-\mathrm{C} 6-\mathrm{H} 6 \mathrm{~A}$ & $110.0(15)$ & $\mathrm{C} 8^{\prime}-\mathrm{C} 7^{\prime}-\mathrm{C} 6^{\prime}$ & $126(3)$ \\
$\mathrm{O} 3-\mathrm{C} 6-\mathrm{H} 6 \mathrm{~B}$ & $109.4(15)$ & $\mathrm{C} 8^{\prime}-\mathrm{C} 7^{\prime}-\mathrm{H} 7^{\prime}$ & $114(3)$ \\
$\mathrm{C} 5-\mathrm{C} 6-\mathrm{H} 6 \mathrm{~A}$ & $107.5(14)$ & $\mathrm{C} 6^{\prime}-\mathrm{C} 7^{\prime}-\mathrm{H} 7^{\prime}$ & \\
$\mathrm{C} 5-\mathrm{C} 6-\mathrm{H} 6 \mathrm{~B}$ & $112.5(15)$ & & \\
\hline
\end{tabular}

2-(2-Methyl-5-nitro-1H-imidazol-1-yl)ethyl benzoate-4-methoxybenzoic acid (1/1) (bzmdmac)

Crystal data

$\mathrm{C}_{13} \mathrm{H}_{13} \mathrm{~N}_{3} \mathrm{O}_{4} \cdot \mathrm{C}_{8} \mathrm{H}_{8} \mathrm{O}_{3}$

$M_{r}=427.41$

Triclinic, $P \overline{1}$

$a=9.1309(4) \AA$

$b=11.1274(4) \AA$

$c=11.8538(5) \AA$

$\alpha=107.365(1)^{\circ}$

$\beta=103.270(1)^{\circ}$

$\gamma=110.091(1)^{\circ}$

$V=1002.73(7) \AA^{3}$

\section{Data collection}

Bruker APEX3 microsource diffractometer

$\varphi$ and $\omega$ scans

Absorption correction: multi-scan

(SADABS; Bruker, 2016)

$T_{\text {min }}=0.587, T_{\max }=0.746$

32021 measured reflections

\section{Refinement}

Refinement on $F^{2}$

Least-squares matrix: full

$R\left[F^{2}>2 \sigma\left(F^{2}\right)\right]=0.059$

$w R\left(F^{2}\right)=0.165$

$S=1.05$

4583 reflections

364 parameters

0 restraints
$Z=2$

$F(000)=448$

$D_{\mathrm{x}}=1.416 \mathrm{Mg} \mathrm{m}^{-3}$

Mo $K \alpha$ radiation, $\lambda=0.71073 \AA$

Cell parameters from 9919 reflections

$\theta=2.5-27.5^{\circ}$

$\mu=0.11 \mathrm{~mm}^{-1}$

$T=300 \mathrm{~K}$

Plate, clear light colourless

$0.73 \times 0.49 \times 0.21 \mathrm{~mm}$

4583 independent reflections

3795 reflections with $I>2 \sigma(I)$

$R_{\text {int }}=0.063$

$\theta_{\max }=27.5^{\circ}, \theta_{\min }=3.6^{\circ}$

$h=-11 \rightarrow 11$

$k=-14 \rightarrow 14$

$l=-15 \rightarrow 15$

Hydrogen site location: difference Fourier map

All H-atom parameters refined

$w=1 /\left[\sigma^{2}\left(F_{\mathrm{o}}^{2}\right)+(0.0785 P)^{2}+0.4129 P\right]$

where $P=\left(F_{\mathrm{o}}{ }^{2}+2 F_{\mathrm{c}}{ }^{2}\right) / 3$

$(\Delta / \sigma)_{\max }<0.001$

$\Delta \rho_{\max }=0.76 \mathrm{e}^{-3}$

$\Delta \rho_{\min }=-0.24$ e $\AA^{-3}$ 


\section{Special details}

Geometry. All esds (except the esd in the dihedral angle between two 1.s. planes) are estimated using the full covariance matrix. The cell esds are taken into account individually in the estimation of esds in distances, angles and torsion angles; correlations between esds in cell parameters are only used when they are defined by crystal symmetry. An approximate (isotropic) treatment of cell esds is used for estimating esds involving l.s. planes.

Refinement. Single-crystal X-ray diffraction data collection ( scans and $\omega$ scans with $\kappa$ and $\theta$ offsets) were performed at room temperature on a Bruker AXS D8 VENTURE equipped with a Kappa goniometer, PHOTON II CPAD detector, a Mo $K \alpha$ INCOATEC I $\mu$ S 3.0 microfocus source $(\lambda=0.71073 \AA)$. The crystal centring, unit-cell determination, refinement of the unit-cell parameters and data collection was controlled through the program APEX3 (Bruker, 2012). The frame integration was performed using SAINT (Bruker, 2016) and the intensities were scaled and absorption corrected using SADABS (Bruker, 2001). Using OLEX2 (Dolomanov et al., 2009), the structure was solved by intrinsic phasing using SHELXT (Sheldrick, 2015) and refined by full-matrix least-squares calculation based on $F^{2}$ for all reflection using SHELXL (Sheldrick, 2007). All non-H atoms were refined anisotropically.

Fractional atomic coordinates and isotropic or equivalent isotropic displacement parameters $\left(\AA^{2}\right)$

\begin{tabular}{|c|c|c|c|c|}
\hline & $x$ & $y$ & $z$ & $U_{\text {iso }} * / U_{\text {eq }}$ \\
\hline $\mathrm{O} 3$ & $0.25372(18)$ & $0.92488(15)$ & $0.54618(12)$ & 0.0501 \\
\hline $\mathrm{O} 1^{\prime}$ & $0.8663(2)$ & 0.60162 (19) & $0.82192(14)$ & $0.0593(4)$ \\
\hline O3' & $1.3864(2)$ & $0.35358(17)$ & $0.84208(14)$ & $0.0603(4)$ \\
\hline $\mathrm{O} 2^{\prime}$ & $0.8625(2)$ & $0.56494(18)$ & $0.62520(14)$ & $0.0617(4)$ \\
\hline $\mathrm{O} 4$ & $0.3621(2)$ & $0.82888(18)$ & $0.41261(14)$ & $0.0592(4)$ \\
\hline $\mathrm{O} 2$ & $0.2647(2)$ & $0.86239(18)$ & $0.88018(15)$ & $0.0624(4)$ \\
\hline $\mathrm{O} 1$ & $0.4097(2)$ & $0.84360(19)$ & $1.03975(14)$ & $0.0675(5)$ \\
\hline N1 & $0.43006(18)$ & $0.75915(15)$ & $0.73271(14)$ & 0.0395 \\
\hline N3 & $0.3715(2)$ & $0.83160(16)$ & $0.92979(14)$ & $0.0442(4)$ \\
\hline $\mathrm{N} 2$ & $0.63325(19)$ & $0.70185(17)$ & $0.79998(15)$ & $0.0463(4)$ \\
\hline $\mathrm{C} 2^{\prime}$ & $1.0933(2)$ & $0.44853(18)$ & $0.66222(16)$ & $0.0404(4)$ \\
\hline $\mathrm{C} 1^{\prime}$ & $1.0392(2)$ & $0.50531(17)$ & $0.75630(15)$ & $0.0372(4)$ \\
\hline $\mathrm{C} 8$ & $0.1692(2)$ & $0.91266(18)$ & $0.33808(16)$ & $0.0395(4)$ \\
\hline $\mathrm{C} 5$ & $0.3002(2)$ & $0.76950(19)$ & $0.64032(17)$ & $0.0423(4)$ \\
\hline $\mathrm{C} 3^{\prime}$ & $1.2086(2)$ & 0.39608 (19) & $0.68643(17)$ & $0.0420(4)$ \\
\hline $\mathrm{C} 3$ & $0.4572(2)$ & $0.77942(18)$ & $0.85787(16)$ & $0.0391(4)$ \\
\hline $\mathrm{C} 4^{\prime}$ & $1.2720(2)$ & 0.40079 (19) & $0.80701(17)$ & $0.0418(4)$ \\
\hline $\mathrm{C} 7$ & $0.2705(2)$ & 0.88207 (19) & $0.43220(17)$ & $0.0420(4)$ \\
\hline $\mathrm{C} 1$ & $0.5419(2)$ & $0.71451(18)$ & $0.70306(17)$ & $0.0403(4)$ \\
\hline$C 7^{\prime}$ & $0.9147(2)$ & $0.55954(19)$ & $0.72677(17)$ & $0.0422(4)$ \\
\hline $\mathrm{C} 6^{\prime}$ & $1.1052(2)$ & $0.5099(2)$ & $0.87698(17)$ & $0.0434(4)$ \\
\hline $\mathrm{C} 2$ & $0.5799(2)$ & $0.7421(2)$ & $0.89668(18)$ & $0.0460(4)$ \\
\hline C6 & $0.3670(3)$ & $0.9180(2)$ & $0.64893(18)$ & $0.0482(4)$ \\
\hline $\mathrm{C} 4$ & $0.5590(3)$ & $0.6836(3)$ & $0.5775(2)$ & $0.0540(5)$ \\
\hline $\mathrm{C} 5^{\prime}$ & $1.2197(3)$ & $0.4587(2)$ & $0.90209(18)$ & $0.0474(4)$ \\
\hline C9 & $0.1616(3)$ & $0.8622(2)$ & $0.21389(18)$ & $0.0508(5)$ \\
\hline $\mathrm{C} 8^{\prime}$ & $1.4517(4)$ & 0.2998 & $0.7517(3)$ & $0.0650(6)$ \\
\hline $\mathrm{C} 13$ & $0.0911(3)$ & $0.9973(3)$ & $0.3722(2)$ & $0.0545(5)$ \\
\hline C11 & $-0.0037(3)$ & $0.9767(3)$ & $0.1587(2)$ & $0.0651(6)$ \\
\hline $\mathrm{C} 10$ & $0.0749(3)$ & $0.8943(3)$ & $0.1246(2)$ & $0.0639(6)$ \\
\hline $\mathrm{C} 12$ & 0.0049 & $1.0293(3)$ & 0.2823 & $0.0670(6)$ \\
\hline H5A & $0.269(3)$ & $0.702(2)$ & $0.552(2)$ & $0.046(5)^{*}$ \\
\hline
\end{tabular}




$\begin{array}{lllll}\text { H5B } & 0.194(3) & 0.744(2) & 0.6573(19) & 0.041(5)^{*} \\ \text { H6A } & 0.480(3) & 0.942(2) & 0.640(2) & 0.055(6)^{*} \\ \text { H6B } & 0.373(3) & 0.985(2) & 0.734(2) & 0.054(6)^{*} \\ \text { H6' } & 1.069(3) & 0.549(2) & 0.941(2) & 0.062(6)^{*} \\ \text { H2' } & 1.055(3) & 0.446(2) & 0.578(2) & 0.051(6)^{*} \\ \text { H4A } & 0.645(3) & 0.659(2) & 0.577(2) & 0.056(6)^{*} \\ \text { H2 } & 0.624(3) & 0.739(3) & 0.977(3) & 0.070(7)^{*} \\ \text { H3' } & 1.243(3) & 0.357(2) & 0.620(2) & 0.062(7)^{*} \\ \text { H5' } & 1.261(3) & 0.460(3) & 0.983(3) & 0.071(7)^{*} \\ \text { H8'A } & 1.509(4) & 0.375(3) & 0.726(3) & 0.081(8)^{*} \\ \text { H9 } & 0.219(3) & 0.804(3) & 0.190(2) & 0.069(7)^{*} \\ \text { H13 } & 0.095(3) & 1.031(3) & 0.451(3) & 0.066(7)^{*} \\ \text { H4B } & 0.458(4) & 0.606(3) & 0.509(3) & 0.090(9)^{*} \\ \text { H1' } & 0.798(4) & 0.636(3) & 0.811(3) & 0.086(9)^{*} \\ \text { H4C } & 0.579(4) & 0.759(3) & 0.556(3) & 0.084(9)^{*} \\ \text { H8'B } & 1.362(4) & 0.219(3) & 0.681(3) & 0.088(9)^{*} \\ \text { H8'C } & 1.527(4) & 0.271(3) & 0.796(3) & 0.079(8)^{*} \\ \text { H10 } & 0.069(4) & 0.855(3) & 0.037(3) & 0.096(9)^{*} \\ \text { H12 } & -0.045(4) & 1.088(3) & 0.313(3) & 0.095(10)^{*} \\ \text { H11 } & -0.073(4) & 0.990(3) & 0.088(3) & 0.082(8)^{*} \\ & & & & \end{array}$

Atomic displacement parameters $\left(\AA^{2}\right)$

\begin{tabular}{lllllll}
\hline & $U^{11}$ & $U^{22}$ & $U^{33}$ & $U^{12}$ & $U^{13}$ & $U^{23}$ \\
\hline O3 & $0.0631(8)$ & $0.0679(8)$ & $0.0405(7)$ & $0.0422(7)$ & $0.0235(6)$ & $0.0306(6)$ \\
O1 $^{\prime}$ & $0.0634(9)$ & $0.0923(11)$ & $0.0499(8)$ & $0.0555(9)$ & $0.0293(7)$ & $0.0337(8)$ \\
O3 $^{\prime}$ & $0.0698(9)$ & $0.0814(10)$ & $0.0511(8)$ & $0.0548(8)$ & $0.0214(7)$ & $0.0307(7)$ \\
O2 $^{\prime}$ & $0.0699(9)$ & $0.0916(11)$ & $0.0458(8)$ & $0.0558(9)$ & $0.0213(7)$ & $0.0333(8)$ \\
O4 & $0.0709(9)$ & $0.0877(10)$ & $0.0554(8)$ & $0.0566(9)$ & $0.0350(7)$ & $0.0419(8)$ \\
O2 & $0.0763(10)$ & $0.0866(11)$ & $0.0602(9)$ & $0.0601(9)$ & $0.0375(8)$ & $0.0386(8)$ \\
O1 & $0.0819(11)$ & $0.0950(12)$ & $0.0368(7)$ & $0.0490(10)$ & $0.0272(7)$ & $0.0260(8)$ \\
N1 & $0.0455(7)$ & $0.0473(8)$ & $0.0370(7)$ & $0.0274(6)$ & $0.0182(6)$ & $0.0213(6)$ \\
N3 & $0.0501(8)$ & $0.0473(8)$ & $0.0407(8)$ & $0.0245(7)$ & $0.0208(7)$ & $0.0187(6)$ \\
N2 & $0.0451(8)$ & $0.0586(9)$ & $0.0472(8)$ & $0.0310(7)$ & $0.0185(7)$ & $0.0265(7)$ \\
C2' & $0.0439(9)$ & $0.0471(9)$ & $0.0325(8)$ & $0.0221(7)$ & $0.0136(7)$ & $0.0177(7)$ \\
C1 & $0.0361(8)$ & $0.0417(8)$ & $0.0364(8)$ & $0.0177(6)$ & $0.0144(6)$ & $0.0178(7)$ \\
C8 & $0.0384(8)$ & $0.0490(9)$ & $0.0381(8)$ & $0.0209(7)$ & $0.0165(7)$ & $0.0235(7)$ \\
C5 & $0.0425(9)$ & $0.0461(9)$ & $0.0400(9)$ & $0.0214(7)$ & $0.0145(7)$ & $0.0187(7)$ \\
C3' & $0.0480(9)$ & $0.0476(9)$ & $0.0355(8)$ & $0.0257(8)$ & $0.0183(7)$ & $0.0162(7)$ \\
C3 & $0.0433(8)$ & $0.0438(8)$ & $0.0353(8)$ & $0.0216(7)$ & $0.0163(7)$ & $0.0186(7)$ \\
C4' & $0.0427(9)$ & $0.0462(9)$ & $0.0405(9)$ & $0.0240(7)$ & $0.0133(7)$ & $0.0194(7)$ \\
C7 & $0.0463(9)$ & $0.0523(9)$ & $0.0405(9)$ & $0.0277(8)$ & $0.0205(7)$ & $0.0256(8)$ \\
C1 & $0.0431(9)$ & $0.0440(9)$ & $0.0417(9)$ & $0.0237(7)$ & $0.0192(7)$ & $0.0201(7)$ \\
C7' & $0.0397(8)$ & $0.0483(9)$ & $0.0399(9)$ & $0.0215(7)$ & $0.0150(7)$ & $0.0174(7)$ \\
C6 & $0.0461(9)$ & $0.0562(10)$ & $0.0371(9)$ & $0.0265(8)$ & $0.0211(7)$ & $0.0226(8)$ \\
C2 & $0.0468(9)$ & $0.0588(11)$ & $0.0402(9)$ & $0.0285(8)$ & $0.0152(7)$ & $0.0249(8)$ \\
C6 & $0.0546(11)$ & $0.0501(10)$ & $0.0388(9)$ & $0.0232(8)$ & $0.0120(8)$ & $0.0209(8)$ \\
C4 & $0.0650(13)$ & $0.0688(13)$ & $0.0497(11)$ & $0.0431(11)$ & $0.0311(10)$ & $0.0287(10)$
\end{tabular}


supporting information

\begin{tabular}{lllllll} 
C5' & $0.0550(10)$ & $0.0630(11)$ & $0.0346(9)$ & $0.0320(9)$ & $0.0178(8)$ & $0.0254(8)$ \\
C9 & $0.0606(11)$ & $0.0591(11)$ & $0.0383(9)$ & $0.0316(10)$ & $0.0186(8)$ & $0.0209(8)$ \\
C8' & $0.0742(15)$ & $0.0746(15)$ & $0.0626(14)$ & $0.0540(14)$ & $0.0248(12)$ & $0.0250(12)$ \\
C13 & $0.0622(12)$ & $0.0804(14)$ & $0.0480(11)$ & $0.0464(11)$ & $0.0302(9)$ & $0.0363(10)$ \\
C11 & $0.0629(13)$ & $0.0952(17)$ & $0.0603(13)$ & $0.0452(13)$ & $0.0218(11)$ & $0.0504(13)$ \\
C10 & $0.0773(15)$ & $0.0840(15)$ & $0.0384(10)$ & $0.0415(13)$ & $0.0187(10)$ & $0.0308(10)$ \\
C12 & $0.0744(15)$ & $0.0998(18)$ & $0.0706(15)$ & $0.0638(14)$ & $0.0384(12)$ & $0.0524(14)$ \\
\hline
\end{tabular}

Geometric parameters $\left(\AA,{ }^{\circ}\right)$

\begin{tabular}{|c|c|c|c|}
\hline $\mathrm{O} 3-\mathrm{C} 7$ & $1.355(2)$ & $\mathrm{C} 3^{\prime}-\mathrm{C} 4^{\prime}$ & $1.391(2)$ \\
\hline $\mathrm{O} 3-\mathrm{C} 6$ & $1.446(2)$ & $\mathrm{C}^{\prime}-\mathrm{H} 3^{\prime}$ & $0.96(3)$ \\
\hline $\mathrm{O} 1^{\prime}-\mathrm{C}^{\prime}$ & $1.320(2)$ & $\mathrm{C} 3-\mathrm{C} 2$ & $1.355(2)$ \\
\hline $\mathrm{O} 1^{\prime}-\mathrm{H} 1^{\prime}$ & $0.84(3)$ & $\mathrm{C} 4^{\prime}-\mathrm{C} 5^{\prime}$ & $1.392(3)$ \\
\hline $\mathrm{O} 3^{\prime}-\mathrm{C}^{\prime}$ & $1.359(2)$ & $\mathrm{C} 1-\mathrm{C} 4$ & $1.482(3)$ \\
\hline $\mathrm{O}^{\prime}-\mathrm{C} 8^{\prime}$ & $1.421(3)$ & $\mathrm{C} 6^{\prime}-\mathrm{C} 5^{\prime}$ & $1.369(3)$ \\
\hline $\mathrm{O} 2^{\prime}-\mathrm{C} 7^{\prime}$ & $1.217(2)$ & $\mathrm{C} 6^{\prime}-\mathrm{H} 6^{\prime}$ & $0.94(3)$ \\
\hline $\mathrm{O} 4-\mathrm{C} 7$ & $1.204(2)$ & $\mathrm{C} 2-\mathrm{H} 2$ & $0.97(3)$ \\
\hline $\mathrm{O} 2-\mathrm{N} 3$ & $1.225(2)$ & C6-H6A & $1.01(2)$ \\
\hline $\mathrm{O} 1-\mathrm{N} 3$ & $1.222(2)$ & C6-H6B & $1.04(2)$ \\
\hline $\mathrm{N} 1-\mathrm{C} 5$ & $1.480(2)$ & $\mathrm{C} 4-\mathrm{H} 4 \mathrm{~A}$ & $0.92(3)$ \\
\hline $\mathrm{N} 1-\mathrm{C} 3$ & $1.381(2)$ & $\mathrm{C} 4-\mathrm{H} 4 \mathrm{~B}$ & $0.97(3)$ \\
\hline $\mathrm{N} 1-\mathrm{C} 1$ & $1.350(2)$ & $\mathrm{C} 4-\mathrm{H} 4 \mathrm{C}$ & $0.92(3)$ \\
\hline $\mathrm{N} 3-\mathrm{C} 3$ & $1.414(2)$ & $\mathrm{C}^{\prime}-\mathrm{H} 5^{\prime}$ & $0.94(3)$ \\
\hline $\mathrm{N} 2-\mathrm{C} 1$ & $1.331(2)$ & $\mathrm{C} 9-\mathrm{C} 10$ & $1.382(3)$ \\
\hline $\mathrm{N} 2-\mathrm{C} 2$ & $1.361(2)$ & C9-H9 & $0.98(3)$ \\
\hline $\mathrm{C} 2^{\prime}-\mathrm{C} 1^{\prime}$ & $1.391(2)$ & $\mathrm{C} 8^{\prime}-\mathrm{H} 8^{\prime} \mathrm{A}$ & $1.00(3)$ \\
\hline $\mathrm{C} 2^{\prime}-\mathrm{C} 3^{\prime}$ & $1.384(2)$ & $\mathrm{C} 8^{\prime}-\mathrm{H} 8^{\prime} \mathrm{B}$ & $0.95(3)$ \\
\hline $\mathrm{C} 2^{\prime}-\mathrm{H} 2^{\prime}$ & $0.97(2)$ & $\mathrm{C} 8^{\prime}-\mathrm{H} 8^{\prime} \mathrm{C}$ & $0.98(3)$ \\
\hline $\mathrm{C} 1^{\prime}-\mathrm{C} 7^{\prime}$ & $1.481(2)$ & $\mathrm{C} 13-\mathrm{C} 12$ & $1.384(3)$ \\
\hline $\mathrm{C} 1^{\prime}-\mathrm{C}^{\prime}$ & $1.397(2)$ & $\mathrm{C} 13-\mathrm{H} 13$ & $0.88(3)$ \\
\hline $\mathrm{C} 8-\mathrm{C} 7$ & $1.478(2)$ & $\mathrm{C} 11-\mathrm{C} 10$ & $1.372(4)$ \\
\hline $\mathrm{C} 8-\mathrm{C} 9$ & $1.386(3)$ & $\mathrm{C} 11-\mathrm{C} 12$ & $1.379(4)$ \\
\hline $\mathrm{C} 8-\mathrm{C} 13$ & $1.388(3)$ & $\mathrm{C} 11-\mathrm{H} 11$ & $1.01(3)$ \\
\hline $\mathrm{C} 5-\mathrm{C} 6$ & $1.513(3)$ & $\mathrm{C} 10-\mathrm{H} 10$ & $0.98(3)$ \\
\hline $\mathrm{C} 5-\mathrm{H} 5 \mathrm{~A}$ & $1.00(2)$ & $\mathrm{C} 12-\mathrm{H} 12$ & $0.95(3)$ \\
\hline $\mathrm{C} 5-\mathrm{H} 5 \mathrm{~B}$ & $1.00(2)$ & & \\
\hline $\mathrm{C} 7-\mathrm{O} 3-\mathrm{C} 6$ & $115.93(14)$ & $\mathrm{C} 1^{\prime}-\mathrm{C} 6^{\prime}-\mathrm{H} 6^{\prime}$ & $118.4(15)$ \\
\hline $\mathrm{C} 7^{\prime}-\mathrm{O} 1^{\prime}-\mathrm{H} 1^{\prime}$ & $117(2)$ & $\mathrm{C} 5^{\prime}-\mathrm{C} 6^{\prime}-\mathrm{C} 1^{\prime}$ & $120.87(17)$ \\
\hline $\mathrm{C} 4^{\prime}-\mathrm{O} 3^{\prime}-\mathrm{C} 8^{\prime}$ & $118.47(17)$ & $\mathrm{C} 5^{\prime}-\mathrm{C} 6^{\prime}-\mathrm{H} 6^{\prime}$ & $120.7(15)$ \\
\hline $\mathrm{C} 3-\mathrm{N} 1-\mathrm{C} 5$ & $130.29(14)$ & $\mathrm{N} 2-\mathrm{C} 2-\mathrm{H} 2$ & $122.3(16)$ \\
\hline $\mathrm{C} 1-\mathrm{N} 1-\mathrm{C} 5$ & $123.92(15)$ & $\mathrm{C} 3-\mathrm{C} 2-\mathrm{N} 2$ & $109.30(16)$ \\
\hline $\mathrm{C} 1-\mathrm{N} 1-\mathrm{C} 3$ & $105.61(14)$ & $\mathrm{C} 3-\mathrm{C} 2-\mathrm{H} 2$ & $128.3(16)$ \\
\hline $\mathrm{O} 2-\mathrm{N} 3-\mathrm{C} 3$ & $119.27(15)$ & $\mathrm{O} 3-\mathrm{C} 6-\mathrm{C} 5$ & $109.66(15)$ \\
\hline $\mathrm{O} 1-\mathrm{N} 3-\mathrm{O} 2$ & $123.58(16)$ & $\mathrm{O} 3-\mathrm{C} 6-\mathrm{H} 6 \mathrm{~A}$ & $109.6(13)$ \\
\hline $\mathrm{O} 1-\mathrm{N} 3-\mathrm{C} 3$ & $117.15(16)$ & $\mathrm{O} 3-\mathrm{C} 6-\mathrm{H} 6 \mathrm{~B}$ & $107.7(12)$ \\
\hline $\mathrm{C} 1-\mathrm{N} 2-\mathrm{C} 2$ & $106.00(15)$ & $\mathrm{C} 5-\mathrm{C} 6-\mathrm{H} 6 \mathrm{~A}$ & $107.2(12)$ \\
\hline
\end{tabular}




\begin{tabular}{|c|c|c|c|}
\hline $\mathrm{C} 1^{\prime}-\mathrm{C} 2^{\prime}-\mathrm{H} 2^{\prime}$ & $121.8(13)$ & $\mathrm{C} 5-\mathrm{C} 6-\mathrm{H} 6 \mathrm{~B}$ & $109.6(12)$ \\
\hline $\mathrm{C} 3^{\prime}-\mathrm{C} 2^{\prime}-\mathrm{C} 1^{\prime}$ & $121.08(16)$ & $\mathrm{H} 6 \mathrm{~A}-\mathrm{C} 6-\mathrm{H} 6 \mathrm{~B}$ & $113.0(18)$ \\
\hline $\mathrm{C} 3^{\prime}-\mathrm{C} 2^{\prime}-\mathrm{H} 2^{\prime}$ & $117.1(13)$ & $\mathrm{C} 1-\mathrm{C} 4-\mathrm{H} 4 \mathrm{~A}$ & $110.3(15)$ \\
\hline $\mathrm{C} 2^{\prime}-\mathrm{C} 1^{\prime}-\mathrm{C} 7^{\prime}$ & $119.62(15)$ & $\mathrm{C} 1-\mathrm{C} 4-\mathrm{H} 4 \mathrm{~B}$ & $112.5(18)$ \\
\hline $\mathrm{C} 2^{\prime}-\mathrm{C} 1^{\prime}-\mathrm{C} 6^{\prime}$ & $118.55(16)$ & $\mathrm{C} 1-\mathrm{C} 4-\mathrm{H} 4 \mathrm{C}$ & $111.9(19)$ \\
\hline $\mathrm{C} 6^{\prime}-\mathrm{C} 1^{\prime}-\mathrm{C} 7^{\prime}$ & $121.83(16)$ & $\mathrm{H} 4 \mathrm{~A}-\mathrm{C} 4-\mathrm{H} 4 \mathrm{~B}$ & $107(2)$ \\
\hline $\mathrm{C} 9-\mathrm{C} 8-\mathrm{C} 7$ & $118.52(16)$ & $\mathrm{H} 4 \mathrm{~A}-\mathrm{C} 4-\mathrm{H} 4 \mathrm{C}$ & $109(2)$ \\
\hline $\mathrm{C} 9-\mathrm{C} 8-\mathrm{C} 13$ & $119.36(17)$ & $\mathrm{H} 4 \mathrm{~B}-\mathrm{C} 4-\mathrm{H} 4 \mathrm{C}$ & $106(3)$ \\
\hline $\mathrm{C} 13-\mathrm{C} 8-\mathrm{C} 7$ & $121.99(16)$ & $\mathrm{C} 4^{\prime}-\mathrm{C} 5^{\prime}-\mathrm{H} 5^{\prime}$ & $119.7(16)$ \\
\hline $\mathrm{N} 1-\mathrm{C} 5-\mathrm{C} 6$ & $108.80(15)$ & $\mathrm{C} 6^{\prime}-\mathrm{C} 5^{\prime}-\mathrm{C} 4^{\prime}$ & $120.21(17)$ \\
\hline $\mathrm{N} 1-\mathrm{C} 5-\mathrm{H} 5 \mathrm{~A}$ & $111.9(12)$ & $\mathrm{C} 6^{\prime}-\mathrm{C} 5^{\prime}-\mathrm{H} 5^{\prime}$ & $120.1(16)$ \\
\hline $\mathrm{N} 1-\mathrm{C} 5-\mathrm{H} 5 \mathrm{~B}$ & $111.0(11)$ & $\mathrm{C} 8-\mathrm{C} 9-\mathrm{H} 9$ & $119.7(15)$ \\
\hline $\mathrm{C} 6-\mathrm{C} 5-\mathrm{H} 5 \mathrm{~A}$ & $109.4(12)$ & $\mathrm{C} 10-\mathrm{C} 9-\mathrm{C} 8$ & $120.3(2)$ \\
\hline $\mathrm{C} 6-\mathrm{C} 5-\mathrm{H} 5 \mathrm{~B}$ & $110.5(11)$ & $\mathrm{C} 10-\mathrm{C} 9-\mathrm{H} 9$ & $120.1(15)$ \\
\hline $\mathrm{H} 5 \mathrm{~A}-\mathrm{C} 5-\mathrm{H} 5 \mathrm{~B}$ & $105.3(16)$ & $\mathrm{O} 3^{\prime}-\mathrm{C} 8^{\prime}-\mathrm{H} 8^{\prime} \mathrm{A}$ & $108.8(17)$ \\
\hline $\mathrm{C} 2^{\prime}-\mathrm{C} 3^{\prime}-\mathrm{C} 4^{\prime}$ & $119.46(16)$ & $\mathrm{O} 3^{\prime}-\mathrm{C} 8^{\prime}-\mathrm{H} 8^{\prime} \mathrm{B}$ & $108.8(19)$ \\
\hline $\mathrm{C} 2^{\prime}-\mathrm{C} 3^{\prime}-\mathrm{H} 3^{\prime}$ & $119.6(15)$ & $\mathrm{O} 3^{\prime}-\mathrm{C} 8^{\prime}-\mathrm{H} 8^{\prime} \mathrm{C}$ & $104.0(17)$ \\
\hline $\mathrm{C} 4^{\prime}-\mathrm{C} 3^{\prime}-\mathrm{H} 3^{\prime}$ & $120.9(15)$ & $\mathrm{H} 8^{\prime} \mathrm{A}-\mathrm{C} 8^{\prime}-\mathrm{H} 8^{\prime} \mathrm{B}$ & $111(3)$ \\
\hline $\mathrm{N} 1-\mathrm{C} 3-\mathrm{N} 3$ & $124.90(15)$ & $\mathrm{H} 8^{\prime} \mathrm{A}-\mathrm{C} 8^{\prime}-\mathrm{H} 8^{\prime} \mathrm{C}$ & $114(2)$ \\
\hline $\mathrm{C} 2-\mathrm{C} 3-\mathrm{N} 1$ & $107.41(15)$ & $\mathrm{H} 8^{\prime} \mathrm{B}-\mathrm{C} 8^{\prime}-\mathrm{H} 8^{\prime} \mathrm{C}$ & $109(2)$ \\
\hline $\mathrm{C} 2-\mathrm{C} 3-\mathrm{N} 3$ & $127.68(16)$ & $\mathrm{C} 8-\mathrm{C} 13-\mathrm{H} 13$ & $120.7(17)$ \\
\hline $\mathrm{O} 3^{\prime}-\mathrm{C}^{\prime}-\mathrm{C} 3^{\prime}$ & $124.69(17)$ & $\mathrm{C} 12-\mathrm{C} 13-\mathrm{C} 8$ & $120.1(2)$ \\
\hline $\mathrm{O} 3^{\prime}-\mathrm{C} 4^{\prime}-\mathrm{C} 5^{\prime}$ & $115.47(16)$ & $\mathrm{C} 12-\mathrm{C} 13-\mathrm{H} 13$ & $119.3(17)$ \\
\hline $\mathrm{C} 3^{\prime}-\mathrm{C} 4^{\prime}-\mathrm{C} 5^{\prime}$ & $119.83(16)$ & $\mathrm{C} 10-\mathrm{C} 11-\mathrm{C} 12$ & $120.4(2)$ \\
\hline $\mathrm{O} 3-\mathrm{C} 7-\mathrm{C} 8$ & $112.45(14)$ & $\mathrm{C} 10-\mathrm{C} 11-\mathrm{H} 11$ & $116.3(16)$ \\
\hline $\mathrm{O} 4-\mathrm{C} 7-\mathrm{O} 3$ & $122.94(16)$ & $\mathrm{C} 12-\mathrm{C} 11-\mathrm{H} 11$ & $123.2(17)$ \\
\hline $\mathrm{O} 4-\mathrm{C} 7-\mathrm{C} 8$ & $124.57(16)$ & $\mathrm{C} 9-\mathrm{C} 10-\mathrm{H} 10$ & $118.5(19)$ \\
\hline $\mathrm{N} 1-\mathrm{C} 1-\mathrm{C} 4$ & $123.52(16)$ & $\mathrm{C} 11-\mathrm{C} 10-\mathrm{C} 9$ & $120.0(2)$ \\
\hline $\mathrm{N} 2-\mathrm{C} 1-\mathrm{N} 1$ & $111.65(15)$ & $\mathrm{C} 11-\mathrm{C} 10-\mathrm{H} 10$ & $121.4(19)$ \\
\hline $\mathrm{N} 2-\mathrm{C} 1-\mathrm{C} 4$ & $124.83(16)$ & $\mathrm{C} 13-\mathrm{C} 12-\mathrm{H} 12$ & $115(2)$ \\
\hline $\mathrm{O} 1^{\prime}-\mathrm{C} 7^{\prime}-\mathrm{C} 1^{\prime}$ & $113.17(15)$ & $\mathrm{C} 11-\mathrm{C} 12-\mathrm{C} 13$ & $119.9(2)$ \\
\hline $\mathrm{O} 2^{\prime}-\mathrm{C}^{\prime}-\mathrm{O} 1^{\prime}$ & $123.39(17)$ & $\mathrm{C} 11-\mathrm{C} 12-\mathrm{H} 12$ & $125(2)$ \\
\hline $\mathrm{O} 2^{\prime}-\mathrm{C}^{\prime}-\mathrm{C} 1^{\prime}$ & $123.44(16)$ & & \\
\hline
\end{tabular}

2-(2-Methyl-5-nitro-1H-imidazol-1-yl)ethyl benzoate-3,5-dinitrobenzoic acid (1/1) (bzmd35dnz)

Crystal data

$\mathrm{C}_{13} \mathrm{H}_{13} \mathrm{~N}_{3} \mathrm{O}_{4} \cdot \mathrm{C}_{7} \mathrm{H}_{4} \mathrm{~N}_{2} \mathrm{O}_{6}$

$M_{r}=487.39$

Monoclinic, $P 2{ }_{1} / c$

$a=21.0988(13) \AA$

$b=8.4322(5) \AA$

$c=12.0341(8) \AA$

$\beta=99.505(2)^{\circ}$

$V=2111.6(2) \AA^{3}$

$Z=4$
$F(000)=1008$

$D_{\mathrm{x}}=1.533 \mathrm{Mg} \mathrm{m}^{-3}$

Mo $K \alpha$ radiation, $\lambda=0.71073 \AA$

Cell parameters from 9941 reflections

$\theta=2.6-27.3^{\circ}$

$\mu=0.13 \mathrm{~mm}^{-1}$

$T=300 \mathrm{~K}$

Plate, clear light colourless

$0.46 \times 0.40 \times 0.06 \mathrm{~mm}$ 


\section{Data collection \\ Bruker APEXII CCD \\ diffractometer \\ $\varphi$ and $\omega$ scans \\ Absorption correction: multi-scan \\ (SADABS; Bruker, 2016) \\ $T_{\min }=0.587, T_{\max }=0.746$ \\ 71598 measured reflections}

\section{Refinement}

Refinement on $F^{2}$

Least-squares matrix: full

$R\left[F^{2}>2 \sigma\left(F^{2}\right)\right]=0.065$

$w R\left(F^{2}\right)=0.213$

$S=1.14$

4820 reflections

385 parameters

0 restraints

Primary atom site location: dual

Hydrogen site location: difference Fourier map
4820 independent reflections

3321 reflections with $I>2 \sigma(I)$

$R_{\text {int }}=0.108$

$\theta_{\max }=27.5^{\circ}, \theta_{\min }=2.6^{\circ}$

$h=-27 \rightarrow 27$

$k=-10 \rightarrow 10$

$l=-15 \rightarrow 15$

All H-atom parameters refined

$w=1 /\left[\sigma^{2}\left(F_{\mathrm{o}}^{2}\right)+(0.0808 P)^{2}+2.0317 P\right]$

where $P=\left(F_{\mathrm{o}}^{2}+2 F_{\mathrm{c}}{ }^{2}\right) / 3$

$(\Delta / \sigma)_{\max }<0.001$

$\Delta \rho_{\max }=0.40 \mathrm{e} \AA^{-3}$

$\Delta \rho_{\min }=-0.35$ e $\AA^{-3}$

Extinction correction: SHELXL2018

(Sheldrick, 2015b),

$\mathrm{Fc}^{*}=\mathrm{kFc}\left[1+0.001 \mathrm{xFc}^{2} \lambda^{3} / \sin (2 \theta)\right]^{-1 / 4}$

Extinction coefficient: 0.026 (4)

Special details

Geometry. All esds (except the esd in the dihedral angle between two 1.s. planes) are estimated using the full covariance matrix. The cell esds are taken into account individually in the estimation of esds in distances, angles and torsion angles; correlations between esds in cell parameters are only used when they are defined by crystal symmetry. An approximate (isotropic) treatment of cell esds is used for estimating esds involving l.s. planes.

Refinement. Single-crystal X-ray diffraction data collection ( scans and $\omega$ scans with $\kappa$ and $\theta$ offsets) were performed at room temperature on a Bruker AXS D8 VENTURE equipped with a Kappa goniometer, PHOTON II CPAD detector, a Mo $K \alpha$ INCOATEC I $\mu$ S 3.0 microfocus source $(\lambda=0.71073 \AA)$. The crystal centring, unit-cell determination, refinement of the unit-cell parameters and data collection was controlled through the program APEX3 (Bruker, 2012). The frame integration was performed using SAINT (Bruker, 2016) and the intensities were scaled and absorption corrected using SADABS (Bruker, 2001). Using OLEX2 (Dolomanov et al., 2009), the structure was solved by intrinsic phasing using SHELXT (Sheldrick, 2015) and refined by full-matrix least-squares calculation based on $F^{2}$ for all reflection using SHELXL (Sheldrick, 2007). All non-H atoms were refined anisotropically.

Fractional atomic coordinates and isotropic or equivalent isotropic displacement parameters $\left(\AA^{2}\right)$

\begin{tabular}{lllll}
\hline & $x$ & $y$ & $z$ & $U_{\text {iso }} * / U_{\text {eq }}$ \\
\hline O3 & $0.88887(9)$ & $0.4296(3)$ & $0.67819(18)$ & $0.0501(5)$ \\
O1 $^{\prime}$ & $0.62334(9)$ & $0.4206(3)$ & $0.8640(2)$ & $0.0519(6)$ \\
O6 $^{\prime}$ & $0.41665(11)$ & $0.3221(3)$ & $0.9795(2)$ & $0.0617(7)$ \\
O2 $^{\prime}$ & $0.62851(10)$ & $0.6000(3)$ & $0.7300(2)$ & $0.0691(8)$ \\
$\mathrm{N} 1$ & $0.84690(10)$ & $0.3243(3)$ & $0.8853(2)$ & $0.0403(5)$ \\
O3' & $0.42660(12)$ & $0.6852(3)$ & $0.4624(2)$ & $0.0659(7)$ \\
O5 $^{\prime}$ & $0.32969(10)$ & $0.4181(4)$ & $0.8826(2)$ & $0.0672(7)$ \\
O4' & $0.33601(11)$ & $0.6115(4)$ & $0.5067(2)$ & $0.0731(8)$ \\
N2 & $0.74971(11)$ & $0.4037(3)$ & $0.9065(2)$ & $0.0485(6)$ \\
N1' & $0.38764(11)$ & $0.3936(3)$ & $0.8979(2)$ & $0.0500(6)$ \\
O2 & $0.95921(11)$ & $0.3723(4)$ & $1.0441(2)$ & $0.0787(9)$ \\
O4 & $0.97119(11)$ & $0.5544(4)$ & $0.6211(3)$ & $0.0766(8)$ \\
N3 & $0.90992(12)$ & $0.4399(4)$ & $1.0593(2)$ & $0.0521(7)$ \\
O1 & $0.90695(14)$ & $0.5286(4)$ & $1.1385(2)$ & $0.0810(9)$
\end{tabular}




$\begin{array}{lllll}\text { N2' } & 0.39462(13) & 0.6237(3) & 0.5270(2) & 0.0533(7) \\ \mathrm{C}^{\prime} & 0.52600(12) & 0.5108(3) & 0.7595(3) & 0.0419(6) \\ \mathrm{C}^{\prime} & 0.42448(12) & 0.4538(3) & 0.8136(3) & 0.0419(6) \\ \mathrm{C}^{\prime} & 0.49473(13) & 0.5679(3) & 0.6577(3) & 0.0437(7) \\ \mathrm{C}^{\prime} & 0.49130(13) & 0.4515(4) & 0.8391(3) & 0.0427(7) \\ \mathrm{C} 1 & 0.78368(13) & 0.3235(4) & 0.8418(3) & 0.0434(7) \\ \text { C3' } & 0.42799(13) & 0.5640(3) & 0.6359(3) & 0.0436(7) \\ \text { C3 } & 0.85199(13) & 0.4118(4) & 0.9835(2) & 0.0424(6) \\ \text { C7' } & 0.59800(13) & 0.5155(4) & 0.7827(3) & 0.0461(7) \\ \text { C2' } & 0.39175(13) & 0.5094(4) & 0.7128(3) & 0.0454(7) \\ \text { C7 } & 0.91451(14) & 0.5258(4) & 0.6079(3) & 0.0493(7) \\ \text { C8 } & 0.86719(15) & 0.5910(4) & 0.5147(3) & 0.0482(7) \\ \text { C5 } & 0.89796(15) & 0.2510(4) & 0.8332(3) & 0.0493(7) \\ \text { C6 } & 0.93347(15) & 0.3681(5) & 0.7725(3) & 0.0523(8) \\ \text { C2 } & 0.79212(14) & 0.4589(4) & 0.9961(3) & 0.0480(7) \\ \text { C11 } & 0.7809(2) & 0.7221(5) & 0.3390(4) & 0.0706(11) \\ \text { C4 } & 0.75565(19) & 0.2433(6) & 0.7357(4) & 0.0639(10) \\ \text { C10 } & 0.75929(19) & 0.6644(5) & 0.4327(4) & 0.0685(10) \\ \text { C9 } & 0.80146(16) & 0.5962(4) & 0.5207(3) & 0.0569(8) \\ \text { C13 } & 0.8889(2) & 0.6517(4) & 0.4218(3) & 0.0613(9) \\ \text { C12 } & 0.8460(2) & 0.7149(5) & 0.3335(3) & 0.0718(11) \\ \text { H4' } & 0.5171(15) & 0.612(4) & 0.593(3) & 0.046(8)^{*} \\ \text { H2' } & 0.3449(16) & 0.512(4) & 0.695(3) & 0.041(8)^{*} \\ \text { H5A } & 0.8774(17) & 0.173(4) & 0.780(3) & 0.057(10)^{*} \\ \text { H6' } & 0.5098(15) & 0.409(4) & 0.912(3) & 0.044(8)^{*} \\ \text { H5B } & 0.9296(17) & 0.201(4) & 0.897(3) & 0.059(10)^{*} \\ \text { H2 } & 0.7782(19) & 0.516(5) & 1.051(3) & 0.066(11)^{*} \\ \text { H6A } & 0.9522(16) & 0.454(4) & 0.825(3) & 0.049(9)^{*} \\ \text { H9 } & 0.7866(16) & 0.548(4) & 0.590(3) & 0.047(9)^{*} \\ \text { H1' } & 0.671(2) & 0.429(5) & 0.882(3) & 0.073(12)^{*} \\ \text { H6B } & 0.9718(18) & 0.319(4) & 0.747(3) & 0.060(10)^{*} \\ \text { H13 } & 0.935(2) & 0.639(6) & 0.419(4) & 0.089(14)^{*} \\ \text { H10 } & 0.714(2) & 0.669(5) & 0.439(4) & 0.084(13)^{*} \\ \text { H12 } & 0.866(2) & 0.751(6) & 0.265(5) & 0.110(17)^{*} \\ \text { H11 } & 0.7488(19) & 0.769(5) & 0.276(3) & 0.067(11)^{*} \\ \text { H4A } & 0.759(2) & 0.131(7) & 0.751(4) & 0.104(17)^{*} \\ \text { H4B } & 0.773(2) & 0.282(5) & 0.668(4) & 0.072(12)^{*} \\ \text { H4C } & 0.711(3) & 0.267(6) & 0.716(4) & 0.099(16)^{*} \\ & & & & \end{array}$

Atomic displacement parameters $\left(\AA^{2}\right)$

\begin{tabular}{lllllll}
\hline & $U^{11}$ & $U^{22}$ & $U^{33}$ & $U^{12}$ & $U^{13}$ & $U^{23}$ \\
\hline O3 & $0.0329(10)$ & $0.0680(14)$ & $0.0492(12)$ & $-0.0043(9)$ & $0.0063(8)$ & $0.0048(10)$ \\
O1' $^{\prime}$ & $0.0273(9)$ & $0.0594(13)$ & $0.0677(14)$ & $0.0030(9)$ & $0.0035(9)$ & $0.0137(11)$ \\
O6 $^{\prime}$ & $0.0479(12)$ & $0.0870(18)$ & $0.0510(13)$ & $0.0040(12)$ & $0.0107(10)$ & $-0.0001(12)$ \\
O2' $^{\prime}$ & $0.0353(11)$ & $0.0812(17)$ & $0.0884(19)$ & $-0.0069(11)$ & $0.0032(11)$ & $0.0323(15)$ \\
N1 & $0.0306(11)$ & $0.0462(13)$ & $0.0442(13)$ & $0.0038(9)$ & $0.0064(9)$ & $0.0033(10)$ \\
O3' $^{\prime}$ & $0.0580(14)$ & $0.0854(18)$ & $0.0528(14)$ & $0.0023(13)$ & $0.0047(11)$ & $0.0062(13)$
\end{tabular}




\begin{tabular}{|c|c|c|c|c|c|c|}
\hline O5' & $0.0304(11)$ & 0.099 (2) & $0.0742(17)$ & $0.0064(11)$ & $0.0144(10)$ & $-0.0028(14)$ \\
\hline O4' & $0.0410(12)$ & $0.095(2)$ & $0.0752(18)$ & $-0.0037(12)$ & $-0.0136(12)$ & $0.0065(15)$ \\
\hline N2 & $0.0296(11)$ & $0.0577(15)$ & $0.0581(16)$ & $0.0034(10)$ & 0.0075 (11) & $0.0069(12)$ \\
\hline N1' & $0.0341(12)$ & $0.0632(16)$ & $0.0539(16)$ & $0.0018(11)$ & $0.0107(11)$ & $-0.0132(13)$ \\
\hline $\mathrm{O} 2$ & $0.0378(12)$ & $0.124(2)$ & $0.0699(17)$ & 0.0138 (14) & $-0.0042(11)$ & $-0.0054(16)$ \\
\hline $\mathrm{O} 4$ & $0.0367(12)$ & $0.104(2)$ & $0.089(2)$ & $-0.0152(13)$ & $0.0115(12)$ & $0.0193(16)$ \\
\hline N3 & $0.0404(13)$ & $0.0696(18)$ & $0.0443(15)$ & $-0.0002(12)$ & $0.0013(11)$ & $0.0053(13)$ \\
\hline $\mathrm{O} 1$ & $0.0725(18)$ & $0.109(2)$ & $0.0557(16)$ & $0.0028(16)$ & $-0.0065(13)$ & $-0.0216(16)$ \\
\hline $\mathrm{N} 2^{\prime}$ & $0.0437(14)$ & $0.0577(16)$ & $0.0536(16)$ & $0.0041(12)$ & $-0.0061(12)$ & $-0.0073(13)$ \\
\hline $\mathrm{C} 5^{\prime}$ & $0.0296(12)$ & $0.0395(14)$ & $0.0551(17)$ & $0.0026(10)$ & $0.0029(11)$ & $-0.0038(13)$ \\
\hline $\mathrm{C} 1^{\prime}$ & $0.0292(12)$ & $0.0481(15)$ & $0.0481(16)$ & $0.0014(11)$ & $0.0054(11)$ & $-0.0098(12)$ \\
\hline $\mathrm{C}^{\prime}$ & $0.0327(13)$ & $0.0443(15)$ & $0.0529(17)$ & $0.0021(11)$ & $0.0033(12)$ & $-0.0045(13)$ \\
\hline $\mathrm{C} 6^{\prime}$ & $0.0321(13)$ & $0.0448(15)$ & $0.0498(17)$ & $0.0014(11)$ & $0.0024(12)$ & $-0.0065(13)$ \\
\hline $\mathrm{C} 1$ & $0.0313(13)$ & $0.0468(15)$ & $0.0514(16)$ & $-0.0034(11)$ & $0.0048(11)$ & $0.0044(13)$ \\
\hline $\mathrm{C} 3^{\prime}$ & $0.0355(14)$ & $0.0448(15)$ & $0.0480(16)$ & $0.0046(11)$ & $0.0001(12)$ & $-0.0053(12)$ \\
\hline $\mathrm{C} 3$ & $0.0347(13)$ & $0.0499(16)$ & $0.0420(15)$ & $0.0029(11)$ & $0.0042(11)$ & $0.0082(12)$ \\
\hline$C 7^{\prime}$ & $0.0333(13)$ & $0.0466(16)$ & $0.0569(18)$ & $-0.0009(12)$ & $0.0029(12)$ & $0.0028(14)$ \\
\hline $\mathrm{C} 2^{\prime}$ & $0.0305(13)$ & $0.0500(16)$ & $0.0542(17)$ & $0.0036(11)$ & $0.0027(12)$ & $-0.0101(14)$ \\
\hline $\mathrm{C} 7$ & $0.0379(14)$ & $0.0588(18)$ & $0.0526(18)$ & $-0.0064(13)$ & $0.0118(13)$ & $-0.0055(14)$ \\
\hline $\mathrm{C} 8$ & $0.0451(15)$ & $0.0503(17)$ & $0.0493(17)$ & $-0.0048(13)$ & $0.0079(13)$ & $-0.0066(13)$ \\
\hline $\mathrm{C} 5$ & $0.0384(15)$ & $0.0557(18)$ & $0.0552(18)$ & $0.0062(13)$ & $0.0119(13)$ & $0.0000(15)$ \\
\hline C6 & $0.0348(14)$ & $0.072(2)$ & $0.0505(18)$ & $0.0036(14)$ & $0.0081(13)$ & $0.0011(16)$ \\
\hline $\mathrm{C} 2$ & $0.0408(15)$ & $0.0587(18)$ & $0.0456(17)$ & $0.0085(13)$ & $0.0100(13)$ & $0.0043(14)$ \\
\hline $\mathrm{C} 11$ & $0.081(3)$ & $0.055(2)$ & $0.067(2)$ & $0.0016(19)$ & $-0.013(2)$ & $-0.0025(18)$ \\
\hline $\mathrm{C} 4$ & $0.050(2)$ & 0.077 (3) & $0.061(2)$ & $-0.0153(18)$ & $0.0006(16)$ & $-0.0085(19)$ \\
\hline $\mathrm{C} 10$ & $0.052(2)$ & $0.063(2)$ & 0.085 (3) & $-0.0061(17)$ & $-0.0068(19)$ & $-0.006(2)$ \\
\hline C9 & $0.0441(17)$ & $0.0586(19)$ & $0.066(2)$ & $-0.0098(14)$ & $0.0048(15)$ & $-0.0023(17)$ \\
\hline $\mathrm{C} 13$ & $0.065(2)$ & $0.059(2)$ & $0.063(2)$ & $0.0035(17)$ & $0.0208(18)$ & $0.0003(17)$ \\
\hline $\mathrm{C} 12$ & $0.103(3)$ & $0.058(2)$ & $0.055(2)$ & $0.007(2)$ & $0.016(2)$ & $0.0025(17)$ \\
\hline
\end{tabular}

Geometric parameters $\left(\AA,{ }^{\circ}\right)$

\begin{tabular}{llll}
\hline $\mathrm{O} 3-\mathrm{C} 7$ & $1.347(4)$ & $\mathrm{C} 6^{\prime}-\mathrm{H} 6^{\prime}$ & $0.97(3)$ \\
$\mathrm{O} 3-\mathrm{C} 6$ & $1.446(4)$ & $\mathrm{C} 1-\mathrm{C} 4$ & $1.478(5)$ \\
$\mathrm{O} 1^{\prime}-\mathrm{C} 7^{\prime}$ & $1.308(4)$ & $\mathrm{C} 3^{\prime}-\mathrm{C} 2^{\prime}$ & $1.374(4)$ \\
$\mathrm{O} 1^{\prime}-\mathrm{H} 1^{\prime}$ & $0.99(4)$ & $\mathrm{C} 3-\mathrm{C} 2$ & $1.356(4)$ \\
$\mathrm{O} 6^{\prime}-\mathrm{N} 1^{\prime}$ & $1.227(4)$ & $\mathrm{C} 2^{\prime}-\mathrm{H} 2^{\prime}$ & $0.98(3)$ \\
$\mathrm{O} 2^{\prime}-\mathrm{C} 7^{\prime}$ & $1.208(4)$ & $\mathrm{C} 7-\mathrm{C} 8$ & $1.479(5)$ \\
$\mathrm{N} 1-\mathrm{C} 1$ & $1.350(3)$ & $\mathrm{C} 8-\mathrm{C} 9$ & $1.401(4)$ \\
$\mathrm{N} 1-\mathrm{C} 3$ & $1.382(4)$ & $\mathrm{C} 8-\mathrm{C} 13$ & $1.375(5)$ \\
$\mathrm{N} 1-\mathrm{C} 5$ & $1.469(4)$ & $\mathrm{C} 5-\mathrm{C} 6$ & $1.501(5)$ \\
$\mathrm{O} 3^{\prime}-\mathrm{N} 2^{\prime}$ & $1.225(4)$ & $\mathrm{C} 5-\mathrm{H} 5 \mathrm{~A}$ & $0.97(4)$ \\
$\mathrm{O} 5^{\prime}-\mathrm{N} 1^{\prime}$ & $1.223(3)$ & $\mathrm{C} 5-\mathrm{H} 5 \mathrm{~B}$ & $1.02(4)$ \\
$\mathrm{O} 4^{\prime}-\mathrm{N} 2^{\prime}$ & $1.225(3)$ & $\mathrm{C} 6-\mathrm{H} 6 \mathrm{~A}$ & $1.00(4)$ \\
$\mathrm{N} 2-\mathrm{C} 1$ & $1.328(4)$ & $\mathrm{C} 6-\mathrm{H} 6 \mathrm{~B}$ & $1.00(4)$ \\
$\mathrm{N} 2-\mathrm{C} 2$ & $1.364(4)$ & $\mathrm{C} 2-\mathrm{H} 2$ & $0.91(4)$ \\
$\mathrm{N} 1^{\prime}-\mathrm{C} 1^{\prime}$ & $1.467(4)$ & $\mathrm{C} 11-\mathrm{C} 10$ & $1.373(6)$ \\
$\mathrm{O} 2-\mathrm{N} 3$ & $1.226(4)$ & $\mathrm{C} 11-\mathrm{C} 12$ & $1.387(6)$
\end{tabular}




\begin{tabular}{|c|c|c|c|}
\hline $\mathrm{O} 4-\mathrm{C} 7$ & $1.204(4)$ & $\mathrm{C} 11-\mathrm{H} 11$ & $1.01(4)$ \\
\hline $\mathrm{N} 3-\mathrm{O} 1$ & $1.221(4)$ & $\mathrm{C} 4-\mathrm{H} 4 \mathrm{~A}$ & $0.97(6)$ \\
\hline $\mathrm{N} 3-\mathrm{C} 3$ & $1.419(4)$ & $\mathrm{C} 4-\mathrm{H} 4 \mathrm{~B}$ & $1.01(4)$ \\
\hline $\mathrm{N} 2^{\prime}-\mathrm{C} 3^{\prime}$ & $1.469(4)$ & $\mathrm{C} 4-\mathrm{H} 4 \mathrm{C}$ & $0.95(5)$ \\
\hline $\mathrm{C} 5^{\prime}-\mathrm{C} 4^{\prime}$ & $1.379(4)$ & $\mathrm{C} 10-\mathrm{C} 9$ & $1.390(5)$ \\
\hline $\mathrm{C} 5^{\prime}-\mathrm{C} 6^{\prime}$ & $1.391(4)$ & $\mathrm{C} 10-\mathrm{H} 10$ & $0.97(5)$ \\
\hline $\mathrm{C} 5^{\prime}-\mathrm{C} 7^{\prime}$ & $1.499(4)$ & C9-H9 & $1.02(3)$ \\
\hline $\mathrm{C} 1^{\prime}-\mathrm{C} 6^{\prime}$ & $1.393(4)$ & $\mathrm{C} 13-\mathrm{C} 12$ & $1.384(6)$ \\
\hline $\mathrm{C} 1^{\prime}-\mathrm{C} 2^{\prime}$ & $1.375(4)$ & $\mathrm{C} 13-\mathrm{H} 13$ & $0.98(4)$ \\
\hline $\mathrm{C} 4^{\prime}-\mathrm{C} 3^{\prime}$ & $1.390(4)$ & $\mathrm{C} 12-\mathrm{H} 12$ & $1.04(5)$ \\
\hline $\mathrm{C} 4^{\prime}-\mathrm{H} 4^{\prime}$ & $1.05(3)$ & & \\
\hline $\mathrm{C} 7-\mathrm{O} 3-\mathrm{C} 6$ & $115.6(2)$ & $\mathrm{O} 3-\mathrm{C} 7-\mathrm{C} 8$ & $114.1(2)$ \\
\hline $\mathrm{C}^{\prime}-\mathrm{O} 1^{\prime}-\mathrm{H} 1^{\prime}$ & $113(2)$ & $\mathrm{O} 4-\mathrm{C} 7-\mathrm{O} 3$ & $122.1(3)$ \\
\hline $\mathrm{C} 1-\mathrm{N} 1-\mathrm{C} 3$ & $105.5(2)$ & $\mathrm{O} 4-\mathrm{C} 7-\mathrm{C} 8$ & $123.8(3)$ \\
\hline $\mathrm{C} 1-\mathrm{N} 1-\mathrm{C} 5$ & $125.4(3)$ & $\mathrm{C} 9-\mathrm{C} 8-\mathrm{C} 7$ & $121.5(3)$ \\
\hline $\mathrm{C} 3-\mathrm{N} 1-\mathrm{C} 5$ & $129.1(2)$ & $\mathrm{C} 13-\mathrm{C} 8-\mathrm{C} 7$ & $118.8(3)$ \\
\hline $\mathrm{C} 1-\mathrm{N} 2-\mathrm{C} 2$ & $106.9(2)$ & $\mathrm{C} 13-\mathrm{C} 8-\mathrm{C} 9$ & $119.7(3)$ \\
\hline $\mathrm{O} 6^{\prime}-\mathrm{N} 1^{\prime}-\mathrm{C} 1^{\prime}$ & $118.2(2)$ & $\mathrm{N} 1-\mathrm{C} 5-\mathrm{C} 6$ & $113.0(3)$ \\
\hline $\mathrm{O} 5^{\prime}-\mathrm{N} 1^{\prime}-\mathrm{O}^{\prime}$ & $123.9(3)$ & $\mathrm{N} 1-\mathrm{C} 5-\mathrm{H} 5 \mathrm{~A}$ & $107(2)$ \\
\hline $\mathrm{O} 5^{\prime}-\mathrm{N} 1^{\prime}-\mathrm{C} 1^{\prime}$ & $117.9(3)$ & $\mathrm{N} 1-\mathrm{C} 5-\mathrm{H} 5 \mathrm{~B}$ & $107(2)$ \\
\hline $\mathrm{O} 2-\mathrm{N} 3-\mathrm{C} 3$ & $119.2(3)$ & $\mathrm{C} 6-\mathrm{C} 5-\mathrm{H} 5 \mathrm{~A}$ & $109(2)$ \\
\hline $\mathrm{O} 1-\mathrm{N} 3-\mathrm{O} 2$ & $123.7(3)$ & $\mathrm{C} 6-\mathrm{C} 5-\mathrm{H} 5 \mathrm{~B}$ & $108(2)$ \\
\hline $\mathrm{O} 1-\mathrm{N} 3-\mathrm{C} 3$ & $117.0(3)$ & $\mathrm{H} 5 \mathrm{~A}-\mathrm{C} 5-\mathrm{H} 5 \mathrm{~B}$ & $113(3)$ \\
\hline $\mathrm{O} 3^{\prime}-\mathrm{N} 2^{\prime}-\mathrm{C} 3^{\prime}$ & $118.5(3)$ & $\mathrm{O} 3-\mathrm{C} 6-\mathrm{C} 5$ & $107.8(3)$ \\
\hline $\mathrm{O} 4^{\prime}-\mathrm{N} 2^{\prime}-\mathrm{O} 3^{\prime}$ & $123.6(3)$ & $\mathrm{O} 3-\mathrm{C} 6-\mathrm{H} 6 \mathrm{~A}$ & 112.3 (19) \\
\hline $\mathrm{O} 4^{\prime}-\mathrm{N} 2^{\prime}-\mathrm{C} 3^{\prime}$ & $117.9(3)$ & $\mathrm{O} 3-\mathrm{C} 6-\mathrm{H} 6 \mathrm{~B}$ & $111(2)$ \\
\hline $\mathrm{C} 4^{\prime}-\mathrm{C} 5^{\prime}-\mathrm{C} 6^{\prime}$ & $120.5(3)$ & $\mathrm{C} 5-\mathrm{C} 6-\mathrm{H} 6 \mathrm{~A}$ & $110.1(19)$ \\
\hline $\mathrm{C} 4^{\prime}-\mathrm{C} 5^{\prime}-\mathrm{C} 7^{\prime}$ & $118.5(3)$ & $\mathrm{C} 5-\mathrm{C} 6-\mathrm{H} 6 \mathrm{~B}$ & $112(2)$ \\
\hline $\mathrm{C} 6^{\prime}-\mathrm{C} 5^{\prime}-\mathrm{C} 7^{\prime}$ & $120.9(3)$ & $\mathrm{H} 6 \mathrm{~A}-\mathrm{C} 6-\mathrm{H} 6 \mathrm{~B}$ & $104(3)$ \\
\hline $\mathrm{C} 6^{\prime}-\mathrm{C} 1^{\prime}-\mathrm{N} 1^{\prime}$ & $118.4(3)$ & $\mathrm{N} 2-\mathrm{C} 2-\mathrm{H} 2$ & $121(2)$ \\
\hline $\mathrm{C} 2^{\prime}-\mathrm{C} 1^{\prime}-\mathrm{N} 1^{\prime}$ & $118.8(2)$ & $\mathrm{C} 3-\mathrm{C} 2-\mathrm{N} 2$ & $108.2(3)$ \\
\hline $\mathrm{C} 2^{\prime}-\mathrm{C} 1^{\prime}-\mathrm{C} 6^{\prime}$ & $122.8(3)$ & $\mathrm{C} 3-\mathrm{C} 2-\mathrm{H} 2$ & $131(3)$ \\
\hline $\mathrm{C} 5^{\prime}-\mathrm{C} 4^{\prime}-\mathrm{C} 3^{\prime}$ & $118.8(3)$ & $\mathrm{C} 10-\mathrm{C} 11-\mathrm{C} 12$ & $119.3(4)$ \\
\hline $\mathrm{C} 5^{\prime}-\mathrm{C} 4^{\prime}-\mathrm{H} 4^{\prime}$ & $125.4(18)$ & $\mathrm{C} 10-\mathrm{C} 11-\mathrm{H} 11$ & $119(2)$ \\
\hline $\mathrm{C} 3^{\prime}-\mathrm{C} 4^{\prime}-\mathrm{H} 4^{\prime}$ & $115.8(18)$ & $\mathrm{C} 12-\mathrm{C} 11-\mathrm{H} 11$ & $122(2)$ \\
\hline $\mathrm{C} 5^{\prime}-\mathrm{C} 6^{\prime}-\mathrm{C} 1^{\prime}$ & $118.2(3)$ & $\mathrm{C} 1-\mathrm{C} 4-\mathrm{H} 4 \mathrm{~A}$ & $106(3)$ \\
\hline $\mathrm{C} 5^{\prime}-\mathrm{C} 6^{\prime}-\mathrm{H} 6^{\prime}$ & $125.2(18)$ & $\mathrm{C} 1-\mathrm{C} 4-\mathrm{H} 4 \mathrm{~B}$ & $114(2)$ \\
\hline $\mathrm{C} 1^{\prime}-\mathrm{C} 6^{\prime}-\mathrm{H} 6^{\prime}$ & $116.6(19)$ & $\mathrm{C} 1-\mathrm{C} 4-\mathrm{H} 4 \mathrm{C}$ & $111(3)$ \\
\hline $\mathrm{N} 1-\mathrm{C} 1-\mathrm{C} 4$ & $124.5(3)$ & $\mathrm{H} 4 \mathrm{~A}-\mathrm{C} 4-\mathrm{H} 4 \mathrm{~B}$ & $116(4)$ \\
\hline $\mathrm{N} 2-\mathrm{C} 1-\mathrm{N} 1$ & $111.3(3)$ & $\mathrm{H} 4 \mathrm{~A}-\mathrm{C} 4-\mathrm{H} 4 \mathrm{C}$ & $107(4)$ \\
\hline $\mathrm{N} 2-\mathrm{C} 1-\mathrm{C} 4$ & $124.2(3)$ & $\mathrm{H} 4 \mathrm{~B}-\mathrm{C} 4-\mathrm{H} 4 \mathrm{C}$ & $102(4)$ \\
\hline $\mathrm{C} 4^{\prime}-\mathrm{C} 3^{\prime}-\mathrm{N} 2^{\prime}$ & $118.8(3)$ & $\mathrm{C} 11-\mathrm{C} 10-\mathrm{C} 9$ & $121.0(4)$ \\
\hline $\mathrm{C} 2^{\prime}-\mathrm{C} 3^{\prime}-\mathrm{N} 2^{\prime}$ & $118.5(3)$ & $\mathrm{C} 11-\mathrm{C} 10-\mathrm{H} 10$ & $121(3)$ \\
\hline $\mathrm{C} 2^{\prime}-\mathrm{C} 3^{\prime}-\mathrm{C} 4^{\prime}$ & $122.7(3)$ & $\mathrm{C} 9-\mathrm{C} 10-\mathrm{H} 10$ & $118(3)$ \\
\hline $\mathrm{N} 1-\mathrm{C} 3-\mathrm{N} 3$ & $125.1(3)$ & $\mathrm{C} 8-\mathrm{C} 9-\mathrm{H} 9$ & 117.9 (19) \\
\hline $\mathrm{C} 2-\mathrm{C} 3-\mathrm{N} 1$ & $108.0(3)$ & $\mathrm{C} 10-\mathrm{C} 9-\mathrm{C} 8$ & $119.2(4)$ \\
\hline $\mathrm{C} 2-\mathrm{C} 3-\mathrm{N} 3$ & $126.7(3)$ & $\mathrm{C} 10-\mathrm{C} 9-\mathrm{H} 9$ & 122.9 (19) \\
\hline
\end{tabular}




$\begin{array}{llll}\mathrm{O} 1^{\prime}-\mathrm{C} 7^{\prime}-\mathrm{C} 5^{\prime} & 113.6(3) & \mathrm{C} 8-\mathrm{C} 13-\mathrm{C} 12 & 120.3(4) \\ \mathrm{O} 2^{\prime}-\mathrm{C} 7^{\prime}-\mathrm{O} 1^{\prime} & 124.5(3) & \mathrm{C} 8-\mathrm{C} 13-\mathrm{H} 13 & 117(3) \\ \mathrm{O}^{\prime}-\mathrm{C} 7^{\prime}-\mathrm{C} 5^{\prime} & 122.0(3) & \mathrm{C} 12-\mathrm{C} 13-\mathrm{H} 13 & 123(3) \\ \mathrm{C} 1^{\prime}-\mathrm{C} 2^{\prime}-\mathrm{H} 2^{\prime} & 122.6(18) & \mathrm{C} 11-\mathrm{C} 12-\mathrm{H} 12 & 124(3) \\ \mathrm{C} 3^{\prime}-\mathrm{C} 2^{\prime}-\mathrm{C} 1^{\prime} & 117.0(3) & \mathrm{C} 13-\mathrm{C} 12-\mathrm{C} 11 & 120.5(4) \\ \mathrm{C} 3^{\prime}-\mathrm{C} 2^{\prime}-\mathrm{H} 2^{\prime} & 120.4(18) & \mathrm{C} 13-\mathrm{C} 12-\mathrm{H} 12 & 115(3)\end{array}$

2-(2-Methyl-5-nitro-1H-imidazol-1-yl)ethyl benzoate-3-aminobenzoic acid (1/1) (bzmdabn)

Crystal data

$\mathrm{C}_{13} \mathrm{H}_{13} \mathrm{~N}_{3} \mathrm{O}_{4} \cdot \mathrm{C}_{7} \mathrm{H}_{7} \mathrm{NO}_{2}$

$M_{r}=412.40$

Triclinic, $P \overline{1}$

$a=7.9316$ (7) $\AA$

$b=11.9585(12) \AA$

$c=12.1978(12) \AA$

$\alpha=76.362(3)^{\circ}$

$\beta=78.927(3)^{\circ}$

$\gamma=79.583(3)^{\circ}$

$V=1092.21(18) \AA^{3}$

\section{Data collection}

Bruker APEXII CCD

diffractometer

$\varphi$ and $\omega$ scans

Absorption correction: multi-scan

(SADABS; Bruker, 2016)

$T_{\min }=0.587, T_{\max }=0.746$

38219 measured reflections

\section{Refinement}

Refinement on $F^{2}$

Least-squares matrix: full

$R\left[F^{2}>2 \sigma\left(F^{2}\right)\right]=0.054$

$w R\left(F^{2}\right)=0.168$

$S=1.00$

5008 reflections

340 parameters

0 restraints

Primary atom site location: dual
$Z=2$

$F(000)=432$

$D_{\mathrm{x}}=1.254 \mathrm{Mg} \mathrm{m}^{-3}$

Mo $K \alpha$ radiation, $\lambda=0.71073 \AA$

Cell parameters from 6174 reflections

$\theta=2.7-29.7^{\circ}$

$\mu=0.09 \mathrm{~mm}^{-1}$

$T=300 \mathrm{~K}$

Plate, clear light colourless

$0.91 \times 0.09 \times 0.05 \mathrm{~mm}$

5008 independent reflections

2711 reflections with $I>2 \sigma(I)$

$R_{\text {int }}=0.090$

$\theta_{\max }=27.5^{\circ}, \theta_{\min }=2.6^{\circ}$

$h=-10 \rightarrow 10$

$k=-15 \rightarrow 15$

$l=-15 \rightarrow 15$

Hydrogen site location: mixed

$\mathrm{H}$ atoms treated by a mixture of independent and constrained refinement

$w=1 /\left[\sigma^{2}\left(F_{\mathrm{o}}^{2}\right)+(0.0722 P)^{2}+0.2049 P\right]$

where $P=\left(F_{\mathrm{o}}^{2}+2 F_{\mathrm{c}}^{2}\right) / 3$

$(\Delta / \sigma)_{\max }<0.001$

$\Delta \rho_{\max }=0.25$ e $\AA^{-3}$

$\Delta \rho_{\min }=-0.19 \mathrm{e} \AA^{-3}$

Special details

Geometry. All esds (except the esd in the dihedral angle between two 1.s. planes) are estimated using the full covariance matrix. The cell esds are taken into account individually in the estimation of esds in distances, angles and torsion angles; correlations between esds in cell parameters are only used when they are defined by crystal symmetry. An approximate (isotropic) treatment of cell esds is used for estimating esds involving 1.s. planes.

Refinement. Single-crystal X-ray diffraction data collection ( scans and $\omega$ scans with $\kappa$ and $\theta$ offsets) were performed at room temperature on a Bruker AXS D8 VENTURE equipped with a Kappa goniometer, PHOTON II CPAD detector, a Mo $K \alpha$ INCOATEC I $\mu \mathrm{S} 3.0$ microfocus source $(\lambda=0.71073 \AA)$. The crystal centring, unit-cell determination, refinement of the unit-cell parameters and data collection was controlled through the program APEX3 (Bruker, 2012). The frame integration was performed using SAINT (Bruker, 2016) and the intensities were scaled and absorption corrected using SADABS (Bruker, 2001). Using OLEX2 (Dolomanov et al., 2009), the structure was solved by intrinsic phasing using SHELXT (Sheldrick, 2015) and refined by full-matrix least-squares calculation based on $F^{2}$ for all reflection using SHELXL (Sheldrick, 2007). All non-H atoms were refined anisotropically. 
Fractional atomic coordinates and isotropic or equivalent isotropic displacement parameters $\left(\AA^{2}\right)$

\begin{tabular}{|c|c|c|c|c|}
\hline & $x$ & $y$ & $z$ & $U_{\text {iso }} * / U_{\text {eq }}$ \\
\hline O1 & $0.4965(2)$ & $-0.14226(17)$ & $0.07569(17)$ & $0.0875(6)$ \\
\hline $\mathrm{O} 2$ & $0.2393(2)$ & $-0.07338(15)$ & $0.03516(16)$ & $0.0810(5)$ \\
\hline $\mathrm{O} 3$ & $0.13769(18)$ & 0.29191 (12) & $0.00673(13)$ & $0.0578(4)$ \\
\hline $\mathrm{O} 4$ & $0.1642(3)$ & $0.33490(16)$ & -0.18307 (15) & $0.0845(6)$ \\
\hline N1 & $0.2025(2)$ & $0.08578(15)$ & $0.17918(14)$ & $0.0541(4)$ \\
\hline N2 & $0.3948(3)$ & $0.0924(2)$ & $0.28556(17)$ & $0.0710(6)$ \\
\hline N3 & $0.3595(3)$ & $-0.07569(17)$ & $0.08576(17)$ & $0.0646(5)$ \\
\hline $\mathrm{C} 1$ & $0.3443(3)$ & 0.00215 (19) & $0.15970(18)$ & $0.0558(5)$ \\
\hline $\mathrm{C} 2$ & 0.4600 & $0.0087(3)$ & $0.2261(2)$ & $0.0691(7)$ \\
\hline $\mathrm{H} 2$ & $0.569(4)$ & $-0.039(2)$ & $0.230(2)$ & $0.083(8)^{*}$ \\
\hline $\mathrm{C} 3$ & $0.2405(3)$ & $0.1382(2)$ & $0.25590(18)$ & $0.0612(6)$ \\
\hline $\mathrm{C} 4$ & $0.1251(4)$ & $0.2346(3)$ & $0.3018(2)$ & $0.0881(8)$ \\
\hline $\mathrm{H} 4 \mathrm{~A}$ & 0.103783 & 0.299389 & 0.240158 & $0.132 *$ \\
\hline H4B & 0.179996 & 0.257855 & 0.354842 & $0.132 *$ \\
\hline $\mathrm{H} 4 \mathrm{C}$ & 0.017095 & 0.208828 & 0.340157 & $0.132 *$ \\
\hline $\mathrm{C} 5$ & $0.0448(3)$ & $0.1185(2)$ & $0.1250(2)$ & $0.0619(6)$ \\
\hline $\mathrm{H} 5 \mathrm{~A}$ & $-0.003(3)$ & $0.051(2)$ & $0.1289(18)$ & $0.063(6)^{*}$ \\
\hline H5B & $-0.040(3)$ & $0.1650(19)$ & $0.1716(19)$ & $0.062(6)^{*}$ \\
\hline C6 & $0.0791(3)$ & $0.1848(2)$ & $0.0046(2)$ & $0.0604(6)$ \\
\hline H6A & $0.170(3)$ & $0.141(2)$ & $-0.0427(19)$ & $0.065(7)^{*}$ \\
\hline H6B & $-0.029(3)$ & $0.204(2)$ & $-0.027(2)$ & $0.070(7)^{*}$ \\
\hline $\mathrm{C} 7$ & $0.1776(3)$ & $0.3604(2)$ & $-0.0960(2)$ & $0.0584(5)$ \\
\hline $\mathrm{C} 8$ & $0.2397(3)$ & 0.46813 (19) & $-0.0895(2)$ & $0.0578(5)$ \\
\hline $\mathrm{C} 9$ & $0.2958(4)$ & $0.5415(2)$ & $-0.1918(3)$ & $0.0776(7)$ \\
\hline H9 & $0.294(3)$ & $0.518(2)$ & $-0.264(2)$ & $0.080(8)^{*}$ \\
\hline $\mathrm{C} 10$ & $0.3595(4)$ & $0.6420(3)$ & -0.1898 & $0.0939(10)$ \\
\hline H10 & $0.397(5)$ & 0.693 (3) & -0.261 & $0.130(12)^{*}$ \\
\hline C11 & $0.3661(4)$ & $0.6693(3)$ & -0.0888 & 0.0907 (9) \\
\hline H11 & $0.415(4)$ & $0.744(3)$ & $-0.091(2)$ & $0.104(9)^{*}$ \\
\hline C12 & $0.3096(3)$ & $0.5983(3)$ & $0.0117(3)$ & $0.0803(8)$ \\
\hline H12 & $0.316(4)$ & $0.613(2)$ & $0.085(3)$ & $0.098(9)^{*}$ \\
\hline C13 & $0.2470(3)$ & $0.4974(2)$ & $0.0121(2)$ & $0.0657(6)$ \\
\hline H13 & $0.210(3)$ & $0.448(2)$ & $0.081(2)$ & $0.075(8)^{*}$ \\
\hline $\mathrm{O} 1^{\prime}$ & $0.4959(2)$ & $0.15720(18)$ & $0.45717(16)$ & $0.0845(6)$ \\
\hline $\mathrm{H} 1^{\prime}$ & $0.474(4)$ & $0.122(3)$ & $0.396(3)$ & $0.122(11)^{*}$ \\
\hline $\mathrm{O} 2^{\prime}$ & $0.7592(3)$ & $0.06517(18)$ & $0.41258(17)$ & $0.0914(6)$ \\
\hline $\mathrm{N} 1^{\prime}$ & $1.0963(3)$ & $0.1476(3)$ & $0.6945(2)$ & $0.0842(7)$ \\
\hline $\mathrm{H} 1^{\prime} \mathrm{A}$ & $1.138(4)$ & $0.198(3)$ & $0.722(3)$ & $0.110(11)^{*}$ \\
\hline $\mathrm{H} 1^{\prime} \mathrm{B}$ & $1.168(4)$ & $0.102(3)$ & $0.652(3)$ & $0.095(10)^{*}$ \\
\hline$C 7^{\prime}$ & $0.6603(3)$ & $0.1271(2)$ & $0.4682(2)$ & $0.0643(6)$ \\
\hline $\mathrm{C} 1^{\prime}$ & $0.7135(3)$ & $0.1792(2)$ & $0.55349(18)$ & $0.0617(6)$ \\
\hline $\mathrm{C} 2^{\prime}$ & $0.6028(4)$ & $0.2643(3)$ & $0.6018(2)$ & $0.0823(8)$ \\
\hline $\mathrm{H} 2^{\prime}$ & $0.478(4)$ & $0.298(2)$ & $0.574(2)$ & $0.103(9)^{*}$ \\
\hline $\mathrm{C} 3^{\prime}$ & $0.6603(4)$ & $0.3099(3)$ & 0.6800 & $0.0954(10)$ \\
\hline H3' & $0.580(4)$ & $0.379(3)$ & $0.712(3)$ & $0.127(11)^{*}$ \\
\hline
\end{tabular}




$\begin{array}{lllll}\mathrm{C}^{\prime} & 0.8236(4) & 0.2728(3) & 0.7085(3) & 0.0847(8) \\ \mathrm{H} 4^{\prime} & 0.872(3) & 0.309(2) & 0.762(2) & 0.093(8)^{*} \\ \mathrm{C}^{\prime} & 0.9352(3) & 0.1877(2) & 0.66121(19) & 0.0648(6) \\ \mathrm{C}^{\prime} & 0.8774(3) & 0.1406(2) & 0.58364(18) & 0.0582(5) \\ \mathrm{H}^{\prime} & 0.955(3) & 0.073(2) & 0.5552(19) & 0.068(7)^{*}\end{array}$

Atomic displacement parameters $\left(\AA^{2}\right)$

\begin{tabular}{|c|c|c|c|c|c|c|}
\hline & $U^{11}$ & $U^{22}$ & $U^{33}$ & $U^{12}$ & $U^{13}$ & $U^{23}$ \\
\hline $\mathrm{O} 1$ & $0.0721(11)$ & $0.0846(13)$ & $0.0996(14)$ & $0.0064(10)$ & $-0.0086(10)$ & $-0.0243(11)$ \\
\hline $\mathrm{O} 2$ & $0.0819(12)$ & $0.0795(12)$ & $0.0981(13)$ & $-0.0162(9)$ & $-0.0341(10)$ & $-0.0308(10)$ \\
\hline $\mathrm{O} 3$ & $0.0616(9)$ & $0.0565(9)$ & $0.0611(9)$ & $-0.0122(7)$ & $-0.0197(7)$ & $-0.0130(7)$ \\
\hline $\mathrm{O} 4$ & $0.1176(15)$ & $0.0857(13)$ & $0.0643(11)$ & $-0.0287(11)$ & $-0.0359(10)$ & $-0.0148(9)$ \\
\hline N1 & $0.0496(10)$ & $0.0614(11)$ & $0.0536(10)$ & $-0.0155(8)$ & $-0.0120(8)$ & $-0.0075(8)$ \\
\hline N2 & $0.0744(13)$ & $0.0885(15)$ & $0.0583(12)$ & $-0.0274(11)$ & $-0.0234(10)$ & $-0.0090(11)$ \\
\hline N3 & $0.0614(12)$ & $0.0627(12)$ & $0.0695(12)$ & $-0.0124(10)$ & $-0.0100(10)$ & $-0.0106(10)$ \\
\hline $\mathrm{C} 1$ & $0.0496(12)$ & $0.0627(13)$ & $0.0567(12)$ & $-0.0138(10)$ & $-0.0131(9)$ & $-0.0073(10)$ \\
\hline $\mathrm{C} 2$ & $0.0572(14)$ & $0.0846(18)$ & $0.0661(15)$ & $-0.0151(13)$ & $-0.0201(12)$ & $-0.0047(13)$ \\
\hline $\mathrm{C} 3$ & $0.0686(14)$ & $0.0683(15)$ & $0.0503(12)$ & $-0.0228(12)$ & $-0.0090(11)$ & $-0.0098(11)$ \\
\hline $\mathrm{C} 4$ & $0.105(2)$ & $0.093(2)$ & $0.0704(17)$ & $-0.0207(17)$ & $-0.0032(15)$ & $-0.0293(15)$ \\
\hline $\mathrm{C} 5$ & $0.0464(12)$ & $0.0662(15)$ & $0.0762(16)$ & $-0.0133(11)$ & $-0.0165(11)$ & $-0.0113(13)$ \\
\hline C6 & $0.0582(13)$ & $0.0589(14)$ & $0.0733(15)$ & $-0.0104(11)$ & $-0.0284(12)$ & $-0.0154(12)$ \\
\hline C7 & $0.0557(12)$ & $0.0619(14)$ & $0.0627(14)$ & $-0.0050(10)$ & $-0.0226(10)$ & $-0.0149(11)$ \\
\hline $\mathrm{C} 8$ & $0.0489(11)$ & $0.0547(13)$ & $0.0701(15)$ & $-0.0012(9)$ & $-0.0143(10)$ & $-0.0142(11)$ \\
\hline C9 & $0.0828(18)$ & $0.0676(17)$ & $0.0798(18)$ & $-0.0069(13)$ & $-0.0178(14)$ & $-0.0089(14)$ \\
\hline $\mathrm{C} 10$ & $0.092(2)$ & $0.0645(18)$ & $0.115(3)$ & $-0.0164(15)$ & $-0.0123(19)$ & 0.0019 (19) \\
\hline C11 & $0.0734(18)$ & $0.0643(18)$ & $0.137(3)$ & $-0.0069(14)$ & $-0.0119(18)$ & $-0.032(2)$ \\
\hline $\mathrm{C} 12$ & $0.0664(16)$ & $0.0819(19)$ & $0.106(2)$ & $-0.0073(14)$ & $-0.0108(15)$ & $-0.0497(18)$ \\
\hline $\mathrm{C} 13$ & $0.0560(13)$ & $0.0693(16)$ & $0.0783(17)$ & $-0.0086(11)$ & $-0.0107(12)$ & $-0.0282(14)$ \\
\hline $\mathrm{O} 1^{\prime}$ & $0.0754(12)$ & $0.1161(15)$ & $0.0759(12)$ & $-0.0193(10)$ & $-0.0249(9)$ & $-0.0324(11)$ \\
\hline $\mathrm{O} 2^{\prime}$ & $0.0919(13)$ & $0.1102(15)$ & $0.0926(13)$ & $-0.0068(11)$ & $-0.0299(11)$ & $-0.0545(12)$ \\
\hline $\mathrm{N} 1^{\prime}$ & $0.0798(16)$ & $0.0992(18)$ & $0.0921(17)$ & $-0.0008(14)$ & $-0.0358(13)$ & $-0.0468(15)$ \\
\hline $\mathrm{C} 7^{\prime}$ & $0.0691(15)$ & $0.0757(16)$ & $0.0531(13)$ & $-0.0201(12)$ & $-0.0146(11)$ & $-0.0113(12)$ \\
\hline $\mathrm{C} 1^{\prime}$ & $0.0692(14)$ & $0.0726(15)$ & $0.0482(12)$ & $-0.0178(12)$ & $-0.0107(10)$ & $-0.0153(11)$ \\
\hline $\mathrm{C} 2^{\prime}$ & $0.0738(17)$ & $0.097(2)$ & $0.0850(18)$ & $0.0000(15)$ & $-0.0232(14)$ & $-0.0374(16)$ \\
\hline $\mathrm{C} 3^{\prime}$ & $0.087(2)$ & $0.115(2)$ & $0.100(2)$ & $0.0085(18)$ & $-0.0258(17)$ & $-0.061(2)$ \\
\hline $\mathrm{C} 4^{\prime}$ & $0.0858(19)$ & $0.100(2)$ & 0.0841 (19) & $-0.0021(16)$ & $-0.0251(15)$ & $-0.0491(17)$ \\
\hline $\mathrm{C} 5^{\prime}$ & $0.0704(15)$ & $0.0771(16)$ & $0.0535(12)$ & $-0.0120(12)$ & $-0.0150(11)$ & $-0.0209(11)$ \\
\hline $\mathrm{C} 6^{\prime}$ & $0.0675(14)$ & $0.0623(14)$ & $0.0478(12)$ & $-0.0125(11)$ & $-0.0107(10)$ & $-0.0132(10)$ \\
\hline
\end{tabular}

Geometric parameters $\left(\AA,{ }^{\circ}\right)$

\begin{tabular}{llll}
\hline $\mathrm{O} 1-\mathrm{N} 3$ & $1.229(3)$ & $\mathrm{C} 9-\mathrm{C} 10$ & $1.393(4)$ \\
$\mathrm{O} 2-\mathrm{N} 3$ & $1.224(2)$ & $\mathrm{C} 10-\mathrm{H} 10$ & $0.96(4)$ \\
$\mathrm{O} 3-\mathrm{C} 6$ & $1.446(3)$ & $\mathrm{C} 10-\mathrm{C} 11$ & $1.360(5)$ \\
$\mathrm{O} 3-\mathrm{C} 7$ & $1.341(3)$ & $\mathrm{C} 11-\mathrm{H} 11$ & $1.02(3)$ \\
$\mathrm{O} 4-\mathrm{C} 7$ & $1.201(3)$ & $\mathrm{C} 11-\mathrm{C} 12$ & $1.364(5)$ \\
$\mathrm{N} 1-\mathrm{C} 1$ & $1.387(3)$ & $\mathrm{C} 12-\mathrm{H} 12$ & $0.96(3)$
\end{tabular}




\begin{tabular}{|c|c|c|c|}
\hline $\mathrm{N} 1-\mathrm{C} 3$ & $1.346(3)$ & $\mathrm{C} 12-\mathrm{C} 13$ & $1.385(4)$ \\
\hline $\mathrm{N} 1-\mathrm{C} 5$ & $1.474(3)$ & $\mathrm{C} 13-\mathrm{H} 13$ & $0.94(3)$ \\
\hline $\mathrm{N} 2-\mathrm{C} 2$ & $1.344(3)$ & $\mathrm{O} 1^{\prime}-\mathrm{H} 1^{\prime}$ & $1.00(4)$ \\
\hline $\mathrm{N} 2-\mathrm{C} 3$ & $1.328(3)$ & $\mathrm{O} 1^{\prime}-\mathrm{C}^{\prime}$ & $1.312(3)$ \\
\hline $\mathrm{N} 3-\mathrm{C} 1$ & $1.417(3)$ & $\mathrm{O} 2^{\prime}-\mathrm{C} 7^{\prime}$ & $1.202(3)$ \\
\hline $\mathrm{C} 1-\mathrm{C} 2$ & $1.358(3)$ & $\mathrm{N} 1^{\prime}-\mathrm{H} 1^{\prime} \mathrm{A}$ & $0.90(3)$ \\
\hline $\mathrm{C} 2-\mathrm{H} 2$ & $0.95(3)$ & $\mathrm{N} 1^{\prime}-\mathrm{H} 1^{\prime} \mathrm{B}$ & $0.89(3)$ \\
\hline $\mathrm{C} 3-\mathrm{C} 4$ & $1.485(4)$ & $\mathrm{N} 1^{\prime}-\mathrm{C} 5^{\prime}$ & $1.383(3)$ \\
\hline $\mathrm{C} 4-\mathrm{H} 4 \mathrm{~A}$ & 0.9600 & $\mathrm{C} 7^{\prime}-\mathrm{C} 1^{\prime}$ & $1.490(3)$ \\
\hline $\mathrm{C} 4-\mathrm{H} 4 \mathrm{~B}$ & 0.9600 & $\mathrm{C} 1^{\prime}-\mathrm{C} 2^{\prime}$ & $1.384(4)$ \\
\hline $\mathrm{C} 4-\mathrm{H} 4 \mathrm{C}$ & 0.9600 & $\mathrm{C} 1^{\prime}-\mathrm{C}^{\prime}$ & $1.388(3)$ \\
\hline $\mathrm{C} 5-\mathrm{H} 5 \mathrm{~A}$ & $0.95(2)$ & $\mathrm{C} 2^{\prime}-\mathrm{H} 2^{\prime}$ & $1.08(3)$ \\
\hline $\mathrm{C} 5-\mathrm{H} 5 \mathrm{~B}$ & $0.98(2)$ & $\mathrm{C} 2^{\prime}-\mathrm{C} 3^{\prime}$ & $1.384(4)$ \\
\hline $\mathrm{C} 5-\mathrm{C} 6$ & $1.495(3)$ & $\mathrm{C} 3^{\prime}-\mathrm{H} 3^{\prime}$ & $1.05(3)$ \\
\hline C6-H6A & $0.99(2)$ & $\mathrm{C} 3^{\prime}-\mathrm{C} 4^{\prime}$ & $1.374(4)$ \\
\hline C6-H6B & $0.97(2)$ & $\mathrm{C} 4^{\prime}-\mathrm{H} 4^{\prime}$ & $1.02(3)$ \\
\hline $\mathrm{C} 7-\mathrm{C} 8$ & $1.484(3)$ & $\mathrm{C} 4^{\prime}-\mathrm{C} 5^{\prime}$ & $1.381(4)$ \\
\hline $\mathrm{C} 8-\mathrm{C} 9$ & $1.391(4)$ & $\mathrm{C} 5^{\prime}-\mathrm{C} 6^{\prime}$ & $1.391(3)$ \\
\hline $\mathrm{C} 8-\mathrm{C} 13$ & $1.379(3)$ & $\mathrm{C} 6^{\prime}-\mathrm{H} 6^{\prime}$ & $1.01(2)$ \\
\hline C9-H9 & $0.98(3)$ & & \\
\hline $\mathrm{C} 7-\mathrm{O} 3-\mathrm{C} 6$ & $115.09(17)$ & $\mathrm{C} 8-\mathrm{C} 9-\mathrm{H} 9$ & $118.2(15)$ \\
\hline $\mathrm{C} 1-\mathrm{N} 1-\mathrm{C} 5$ & $129.03(19)$ & $\mathrm{C} 8-\mathrm{C} 9-\mathrm{C} 10$ & $119.7(3)$ \\
\hline $\mathrm{C} 3-\mathrm{N} 1-\mathrm{C} 1$ & $105.68(18)$ & $\mathrm{C} 10-\mathrm{C} 9-\mathrm{H} 9$ & $122.1(15)$ \\
\hline $\mathrm{C} 3-\mathrm{N} 1-\mathrm{C} 5$ & $125.2(2)$ & $\mathrm{C} 9-\mathrm{C} 10-\mathrm{H} 10$ & $119(2)$ \\
\hline $\mathrm{C} 3-\mathrm{N} 2-\mathrm{C} 2$ & $106.95(19)$ & $\mathrm{C} 11-\mathrm{C} 10-\mathrm{C} 9$ & $120.5(3)$ \\
\hline $\mathrm{O} 1-\mathrm{N} 3-\mathrm{C} 1$ & $116.9(2)$ & $\mathrm{C} 11-\mathrm{C} 10-\mathrm{H} 10$ & $121(2)$ \\
\hline $\mathrm{O} 2-\mathrm{N} 3-\mathrm{O} 1$ & $123.5(2)$ & $\mathrm{C} 10-\mathrm{C} 11-\mathrm{H} 11$ & $117.9(17)$ \\
\hline $\mathrm{O} 2-\mathrm{N} 3-\mathrm{C} 1$ & $119.6(2)$ & $\mathrm{C} 10-\mathrm{C} 11-\mathrm{C} 12$ & $120.1(3)$ \\
\hline $\mathrm{N} 1-\mathrm{C} 1-\mathrm{N} 3$ & $125.25(18)$ & $\mathrm{C} 12-\mathrm{C} 11-\mathrm{H} 11$ & $122.0(17)$ \\
\hline $\mathrm{C} 2-\mathrm{C} 1-\mathrm{N} 1$ & $106.9(2)$ & $\mathrm{C} 11-\mathrm{C} 12-\mathrm{H} 12$ & $122.7(18)$ \\
\hline $\mathrm{C} 2-\mathrm{C} 1-\mathrm{N} 3$ & $127.8(2)$ & $\mathrm{C} 11-\mathrm{C} 12-\mathrm{C} 13$ & $120.6(3)$ \\
\hline $\mathrm{N} 2-\mathrm{C} 2-\mathrm{C} 1$ & $109.2(2)$ & $\mathrm{C} 13-\mathrm{C} 12-\mathrm{H} 12$ & $116.7(18)$ \\
\hline $\mathrm{N} 2-\mathrm{C} 2-\mathrm{H} 2$ & $125.6(16)$ & $\mathrm{C} 8-\mathrm{C} 13-\mathrm{C} 12$ & $120.2(3)$ \\
\hline $\mathrm{C} 1-\mathrm{C} 2-\mathrm{H} 2$ & $125.2(16)$ & $\mathrm{C} 8-\mathrm{C} 13-\mathrm{H} 13$ & $119.1(15)$ \\
\hline $\mathrm{N} 1-\mathrm{C} 3-\mathrm{C} 4$ & $124.6(2)$ & $\mathrm{C} 12-\mathrm{C} 13-\mathrm{H} 13$ & $120.7(16)$ \\
\hline $\mathrm{N} 2-\mathrm{C} 3-\mathrm{N} 1$ & $111.2(2)$ & $\mathrm{C} 7^{\prime}-\mathrm{O} 1^{\prime}-\mathrm{H} 1^{\prime}$ & $108.1(19)$ \\
\hline $\mathrm{N} 2-\mathrm{C} 3-\mathrm{C} 4$ & $124.2(2)$ & $\mathrm{H} 1^{\prime} \mathrm{A}-\mathrm{N} 1^{\prime}-\mathrm{H} 1^{\prime} \mathrm{B}$ & $120(3)$ \\
\hline $\mathrm{C} 3-\mathrm{C} 4-\mathrm{H} 4 \mathrm{~A}$ & 109.5 & $\mathrm{C} 5^{\prime}-\mathrm{N} 1^{\prime}-\mathrm{H} 1^{\prime} \mathrm{A}$ & $114(2)$ \\
\hline $\mathrm{C} 3-\mathrm{C} 4-\mathrm{H} 4 \mathrm{~B}$ & 109.5 & $\mathrm{C} 5^{\prime}-\mathrm{N} 1^{\prime}-\mathrm{H} 1^{\prime} \mathrm{B}$ & $115.7(19)$ \\
\hline $\mathrm{C} 3-\mathrm{C} 4-\mathrm{H} 4 \mathrm{C}$ & 109.5 & $\mathrm{O} 1^{\prime}-\mathrm{C} 7^{\prime}-\mathrm{C} 1^{\prime}$ & $113.7(2)$ \\
\hline $\mathrm{H} 4 \mathrm{~A}-\mathrm{C} 4-\mathrm{H} 4 \mathrm{~B}$ & 109.5 & $\mathrm{O} 2^{\prime}-\mathrm{C}^{\prime}-\mathrm{O} 1^{\prime}$ & $122.9(2)$ \\
\hline $\mathrm{H} 4 \mathrm{~A}-\mathrm{C} 4-\mathrm{H} 4 \mathrm{C}$ & 109.5 & $\mathrm{O} 2^{\prime}-\mathrm{C}^{\prime}-\mathrm{C} 1^{\prime}$ & $123.3(2)$ \\
\hline $\mathrm{H} 4 \mathrm{~B}-\mathrm{C} 4-\mathrm{H} 4 \mathrm{C}$ & 109.5 & $\mathrm{C} 2^{\prime}-\mathrm{C} 1^{\prime}-\mathrm{C} 7^{\prime}$ & $121.1(2)$ \\
\hline $\mathrm{N} 1-\mathrm{C} 5-\mathrm{H} 5 \mathrm{~A}$ & $108.9(13)$ & $\mathrm{C} 2^{\prime}-\mathrm{C} 1^{\prime}-\mathrm{C} 6^{\prime}$ & $120.4(2)$ \\
\hline $\mathrm{N} 1-\mathrm{C} 5-\mathrm{H} 5 \mathrm{~B}$ & $107.3(13)$ & $\mathrm{C} 6^{\prime}-\mathrm{C} 1^{\prime}-\mathrm{C} 7^{\prime}$ & $118.5(2)$ \\
\hline $\mathrm{N} 1-\mathrm{C} 5-\mathrm{C} 6$ & $112.39(18)$ & $\mathrm{C} 1^{\prime}-\mathrm{C} 2^{\prime}-\mathrm{H} 2^{\prime}$ & $120.0(16)$ \\
\hline $\mathrm{H} 5 \mathrm{~A}-\mathrm{C} 5-\mathrm{H} 5 \mathrm{~B}$ & $106.2(19)$ & $\mathrm{C} 1^{\prime}-\mathrm{C} 2^{\prime}-\mathrm{C} 3^{\prime}$ & $118.3(3)$ \\
\hline
\end{tabular}




$\begin{array}{llll}\mathrm{C} 6-\mathrm{C} 5-\mathrm{H} 5 \mathrm{~A} & 111.2(13) & \mathrm{C} 3^{\prime}-\mathrm{C} 2^{\prime}-\mathrm{H} 2^{\prime} & 121.6(16) \\ \mathrm{C} 6-\mathrm{C} 5-\mathrm{H} 5 \mathrm{~B} & 110.6(13) & \mathrm{C} 2^{\prime}-\mathrm{C} 3^{\prime}-\mathrm{H} 3^{\prime} & 117.9(19) \\ \mathrm{O} 3-\mathrm{C} 6-\mathrm{C} 5 & 107.92(19) & \mathrm{C} 4^{\prime}-\mathrm{C} 3^{\prime}-\mathrm{C} 2^{\prime} & 121.2(3) \\ \mathrm{O} 3-\mathrm{C} 6-\mathrm{H} 6 \mathrm{~A} & 108.7(13) & \mathrm{C} 4^{\prime}-\mathrm{C} 3^{\prime}-\mathrm{H} 3^{\prime} & 120.7(19) \\ \mathrm{O} 3-\mathrm{C} 6-\mathrm{H} 6 \mathrm{~B} & 108.6(14) & \mathrm{C} 3^{\prime}-\mathrm{C} 4^{\prime}-\mathrm{H} 4^{\prime} & 122.7(15) \\ \mathrm{C} 5-\mathrm{C} 6-\mathrm{H} 6 \mathrm{~A} & 111.5(13) & \mathrm{C} 3^{\prime}-\mathrm{C} 4^{\prime}-\mathrm{C} 5^{\prime} & 121.1(3) \\ \mathrm{C} 5-\mathrm{C} 6-\mathrm{H} 6 \mathrm{~B} & 108.9(13) & \mathrm{C} 5^{\prime}-\mathrm{C} 4^{\prime}-\mathrm{H} 4^{\prime} & 116.1(15) \\ \mathrm{H} 6 \mathrm{~A}-\mathrm{C} 6-\mathrm{H} 6 \mathrm{~B} & 111.2(19) & \mathrm{N} 1^{\prime}-\mathrm{C} 5^{\prime}-\mathrm{C} 6^{\prime} & 121.5(2) \\ \mathrm{O} 3-\mathrm{C} 7-\mathrm{C} 8 & 113.10(19) & \mathrm{C} 4^{\prime}-\mathrm{C} 5^{\prime}-\mathrm{N} 1^{\prime} & 120.5(2) \\ \mathrm{O} 4-\mathrm{C} 7-\mathrm{O} 3 & 122.3(2) & \mathrm{C} 4^{\prime}-\mathrm{C} 5^{\prime}-\mathrm{C} 6^{\prime} & 118.0(2) \\ \mathrm{O} 4-\mathrm{C} 7-\mathrm{C} 8 & 124.6(2) & \mathrm{C} 1^{\prime}-\mathrm{C} 6^{\prime}-\mathrm{C} 5^{\prime} & 121.0(2) \\ \mathrm{C} 9-\mathrm{C} 8-\mathrm{C} 7 & 117.7(2) & \mathrm{C} 1^{\prime}-\mathrm{C} 6^{\prime}-\mathrm{H} 6^{\prime} & 121.5(13) \\ \mathrm{C} 13-\mathrm{C} 8-\mathrm{C} 7 & 123.3(2) & \mathrm{C} 5^{\prime}-\mathrm{C} 6^{\prime}-\mathrm{H} 6^{\prime} & 117.4(13) \\ \mathrm{C} 13-\mathrm{C} 8-\mathrm{C} 9 & 119.0(2) & & \end{array}$

2-(2-Methyl-5-nitro-1 H-imidazol-1-yl)ethyl benzoate-salicylic acid (1/1) (bzmdslc)

\section{Crystal data}

$\mathrm{C}_{13} \mathrm{H}_{13} \mathrm{~N}_{3} \mathrm{O}_{4} \cdot \mathrm{C}_{7} \mathrm{H}_{6} \mathrm{O}_{3}$

$M_{r}=413.38$

Monoclinic, $P 2_{1} / c$

$a=9.5055(6) \AA$

$b=31.2239(19) \AA$

$c=6.8578(4) \AA$

$\beta=102.628(2)^{\circ}$

$V=1986.2(2) \AA^{3}$

$Z=4$

\section{Data collection}

Bruker D8 VENTURE Kappa Duo PHOTON II CPAD

diffractometer

Multilayer mirrors monochromator

$\varphi$ and $\omega$ scans

Absorption correction: multi-scan

(SADABS; Bruker, 2016)

$T_{\min }=0.706, T_{\max }=0.746$

\section{Refinement}

Refinement on $F^{2}$

Least-squares matrix: full

$R\left[F^{2}>2 \sigma\left(F^{2}\right)\right]=0.042$

$w R\left(F^{2}\right)=0.115$

$S=1.06$

4553 reflections

347 parameters

100 restraints
$F(000)=864$

$D_{\mathrm{x}}=1.382 \mathrm{Mg} \mathrm{m}^{-3}$

Mo $K \alpha$ radiation, $\lambda=0.71073 \AA$

Cell parameters from 9833 reflections

$\theta=2.6-27.4^{\circ}$

$\mu=0.11 \mathrm{~mm}^{-1}$

$T=301 \mathrm{~K}$

Prismatic, colourless

$0.68 \times 0.24 \times 0.16 \mathrm{~mm}$

69959 measured reflections

4553 independent reflections

3883 reflections with $I>2 \sigma(I)$

$R_{\text {int }}=0.035$

$\theta_{\max }=27.5^{\circ}, \theta_{\min }=2.9^{\circ}$

$h=-12 \rightarrow 12$

$k=-40 \rightarrow 40$

$l=-8 \rightarrow 8$

Primary atom site location: dual

Hydrogen site location: difference Fourier map

All H-atom parameters refined

$w=1 /\left[\sigma^{2}\left(F_{\mathrm{o}}^{2}\right)+(0.0521 P)^{2}+0.3493 P\right]$

where $P=\left(F_{\mathrm{o}}{ }^{2}+2 F_{\mathrm{c}}{ }^{2}\right) / 3$

$(\Delta / \sigma)_{\max }=0.001$

$\Delta \rho_{\max }=0.17 \mathrm{e} \AA^{-3}$

$\Delta \rho_{\min }=-0.20 \mathrm{e} \AA^{-3}$

Special details

Geometry. All esds (except the esd in the dihedral angle between two 1.s. planes) are estimated using the full covariance matrix. The cell esds are taken into account individually in the estimation of esds in distances, angles and torsion angles; correlations between esds in cell parameters are only used when they are defined by crystal symmetry. An approximate (isotropic) treatment of cell esds is used for estimating esds involving 1.s. planes. 
Refinement. Single-crystal X-ray diffraction data collection ( scans and $\omega$ scans with $\kappa$ and $\theta$ offsets) were performed at room temperature on a Bruker AXS D8 VENTURE equipped with a Kappa goniometer, PHOTON II CPAD detector, a Mo $K \alpha$ INCOATEC I $\mu$ S 3.0 microfocus source $(\lambda=0.71073 \AA$ ). The crystal centring, unit-cell determination, refinement of the unit-cell parameters and data collection was controlled through the program APEX3 (Bruker, 2012). The frame integration was performed using SAINT (Bruker, 2016) and the intensities were scaled and absorption corrected using SADABS (Bruker, 2001). Using OLEX2 (Dolomanov et al., 2009), the structure was solved by intrinsic phasing using SHELXT (Sheldrick, 2015) and refined by full-matrix least-squares calculation based on $F^{2}$ for all reflection using SHELXL (Sheldrick, 2007). All non-H atoms were refined anisotropically.

Fractional atomic coordinates and isotropic or equivalent isotropic displacement parameters $\left(\hat{A}^{2}\right)$

\begin{tabular}{|c|c|c|c|c|}
\hline & $x$ & $y$ & $z$ & $U_{\text {iso }} * / U_{\text {eq }}$ \\
\hline $\mathrm{O} 1$ & $0.87803(16)$ & $0.63094(4)$ & 0.3909 (2) & $0.0887(4)$ \\
\hline $\mathrm{O} 2$ & $0.92555(13)$ & $0.66308(4)$ & $0.67677(19)$ & $0.0803(3)$ \\
\hline $\mathrm{O} 3$ & $0.56700(10)$ & $0.71973(3)$ & $0.84768(15)$ & $0.0583(2)$ \\
\hline $\mathrm{O} 4$ & $0.64060(13)$ & $0.78754(3)$ & $0.84757(19)$ & $0.0771(3)$ \\
\hline N1 & $0.66295(11)$ & $0.63293(3)$ & $0.75126(15)$ & $0.0468(2)$ \\
\hline N2 & $0.52072(13)$ & $0.58617(4)$ & $0.56128(18)$ & $0.0598(3)$ \\
\hline N3 & $0.84921(13)$ & $0.64050(4)$ & $0.55066(19)$ & $0.0603(3)$ \\
\hline $\mathrm{C} 1$ & 0.54094 (14) & $0.60942(4)$ & $0.7272(2)$ & $0.0536(3)$ \\
\hline $\mathrm{C} 2$ & $0.63221(16)$ & $0.59466(4)$ & $0.4743(2)$ & $0.0564(3)$ \\
\hline $\mathrm{H} 2$ & $0.6423(17)$ & $0.5809(6)$ & $0.355(3)$ & $0.073(5)^{*}$ \\
\hline $\mathrm{C} 3$ & $0.71994(14)$ & $0.62349(4)$ & $0.58763(18)$ & $0.0480(3)$ \\
\hline $\mathrm{C} 4$ & $0.4412(2)$ & $0.60928(8)$ & $0.8662(4)$ & $0.0823(5)$ \\
\hline $\mathrm{H} 4 \mathrm{~A}$ & $0.478(3)$ & $0.5934(11)$ & $0.988(5)$ & $0.159(11)^{*}$ \\
\hline $\mathrm{H} 4 \mathrm{~B}$ & $0.427(3)$ & $0.6365(9)$ & $0.916(4)$ & $0.135(10)^{*}$ \\
\hline $\mathrm{H} 4 \mathrm{C}$ & $0.360(4)$ & $0.5953(10)$ & $0.805(5)$ & $0.156(11)^{*}$ \\
\hline $\mathrm{C} 5$ & $0.72381(16)$ & $0.65981(5)$ & $0.92458(19)$ & $0.0558(3)$ \\
\hline $\mathrm{H} 5 \mathrm{~A}$ & $0.8236(17)$ & $0.6512(5)$ & 0.969 (2) & $0.058(4)^{*}$ \\
\hline $\mathrm{H} 5 \mathrm{~B}$ & $0.6706(17)$ & $0.6528(5)$ & $1.028(2)$ & $0.064(4)^{*}$ \\
\hline C6 & $0.71571(16)$ & $0.70700(5)$ & $0.8807(2)$ & $0.0588(3)$ \\
\hline H6A & $0.7540(17)$ & $0.7136(5)$ & $0.761(3)$ & $0.068(4)^{*}$ \\
\hline $\mathrm{C} 7$ & $0.54390(15)$ & $0.76221(4)$ & $0.83503(18)$ & $0.0529(3)$ \\
\hline $\mathrm{O} 1^{\prime}$ & $0.30972(14)$ & $0.53009(4)$ & $0.43561(19)$ & $0.0822(4)$ \\
\hline $\mathrm{H} 1^{\prime}$ & $0.387(2)$ & $0.5478(7)$ & $0.473(3)$ & $0.102(7)^{*}$ \\
\hline $\mathrm{O} 2^{\prime}$ & $0.39947(13)$ & $0.51848(4)$ & $0.16738(19)$ & $0.0785(3)$ \\
\hline O3' & $0.26597(14)$ & $0.46438(4)$ & $-0.10302(19)$ & $0.0787(3)$ \\
\hline H3' & $0.333(3)$ & $0.4843(7)$ & $-0.043(3)$ & $0.105(7)^{*}$ \\
\hline $\mathrm{C} 7^{\prime}$ & $0.30950(15)$ & $0.51100(4)$ & $0.2653(2)$ & $0.0590(3)$ \\
\hline $\mathrm{C} 1^{\prime}$ & $0.18997(14)$ & $0.48065(4)$ & $0.2017(2)$ & $0.0519(3)$ \\
\hline $\mathrm{C} 22^{\prime}$ & $0.17227(15)$ & $0.45986(4)$ & $0.0179(2)$ & $0.0565(3)$ \\
\hline $\mathrm{C} 3^{\prime}$ & $0.05451(17)$ & $0.43294(5)$ & $-0.0447(3)$ & 0.0659 (4) \\
\hline H3'A & $0.0427(19)$ & $0.4198(6)$ & $-0.172(3)$ & $0.080(5)^{*}$ \\
\hline $\mathrm{C}^{\prime}$ & $-0.04243(17)$ & $0.42646(5)$ & $0.0733(3)$ & $0.0686(4)$ \\
\hline $\mathrm{H} 4^{\prime}$ & $-0.127(2)$ & $0.4075(6)$ & $0.022(3)$ & $0.085(5)^{*}$ \\
\hline $\mathrm{C} 5^{\prime}$ & $-0.02454(16)$ & $0.44612(5)$ & $0.2583(3)$ & $0.0668(4)$ \\
\hline $\mathrm{H} 5^{\prime}$ & $-0.091(2)$ & $0.4411(6)$ & $0.340(3)$ & $0.088(6)^{*}$ \\
\hline $\mathrm{C} 6^{\prime}$ & $0.09104(15)$ & $0.47294(5)$ & $0.3210(2)$ & $0.0597(3)$ \\
\hline $\mathrm{H} 6^{\prime}$ & $0.1065(17)$ & $0.4880(5)$ & $0.456(3)$ & $0.073(5)^{*}$ \\
\hline
\end{tabular}




\begin{tabular}{lllll} 
C9 & $0.28162(16)$ & $0.74263(5)$ & $0.7797(2)$ & $0.0616(3)$ \\
C10 & $0.1392(2)$ & $0.75472(7)$ & $0.7613(3)$ & $0.0787(5)$ \\
C11 & $0.1046(2)$ & $0.79697(7)$ & $0.7762(3)$ & $0.0899(6)$ \\
C12 & $0.2100(3)$ & $0.82743(7)$ & $0.8071(3)$ & $0.0932(6)$ \\
C13 & $0.3531(2)$ & $0.81613(5)$ & $0.8229(2)$ & $0.0733(4)$ \\
C8 & $0.38974(16)$ & $0.77321(4)$ & $0.81035(17)$ & $0.0544(3)$ \\
H6B & $0.7712(18)$ & $0.7223(5)$ & $0.994(3)$ & $0.069(4)^{*}$ \\
H13 & $0.4267(19)$ & $0.8361(6)$ & $0.839(3)$ & $0.072(5)^{*}$ \\
H9 & $0.3077(18)$ & $0.7123(5)$ & $0.771(2)$ & $0.070(5)^{*}$ \\
H12 & $0.190(3)$ & $0.8559(8)$ & $0.811(4)$ & $0.123(8)^{*}$ \\
H10 & $0.062(3)$ & $0.7333(8)$ & $0.741(3)$ & $0.112(7)^{*}$ \\
H11 & $0.000(3)$ & $0.8054(7)$ & $0.766(3)$ & $0.116(7)^{*}$ \\
\hline
\end{tabular}

Atomic displacement parameters $\left(\AA^{2}\right)$

\begin{tabular}{|c|c|c|c|c|c|c|}
\hline & $U^{11}$ & $U^{22}$ & $U^{33}$ & $U^{12}$ & $U^{13}$ & $U^{23}$ \\
\hline $\mathrm{O} 1$ & $0.1115(10)$ & $0.0842(8)$ & $0.0896(8)$ & $-0.0158(7)$ & $0.0642(8)$ & $-0.0120(6)$ \\
\hline $\mathrm{O} 2$ & $0.0697(7)$ & $0.0785(7)$ & $0.0976(8)$ & $-0.0226(6)$ & $0.0291(6)$ & $-0.0181(6)$ \\
\hline $\mathrm{O} 3$ & $0.0585(5)$ & $0.0463(5)$ & $0.0674(6)$ & $-0.0003(4)$ & 0.0077 (4) & -0.0079 (4) \\
\hline $\mathrm{O} 4$ & $0.0822(7)$ & $0.0528(6)$ & $0.0979(8)$ & $-0.0125(5)$ & $0.0230(6)$ & $-0.0080(5)$ \\
\hline N1 & $0.0541(6)$ & $0.0414(5)$ & $0.0460(5)$ & $0.0006(4)$ & $0.0134(4)$ & $0.0012(4)$ \\
\hline $\mathrm{N} 2$ & $0.0613(7)$ & $0.0471(6)$ & $0.0695(7)$ & $-0.0066(5)$ & $0.0107(5)$ & $-0.0016(5)$ \\
\hline N3 & $0.0680(7)$ & $0.0500(6)$ & 0.0698 (7) & $-0.0058(5)$ & $0.0300(6)$ & $-0.0020(5)$ \\
\hline $\mathrm{C} 1$ & $0.0541(7)$ & $0.0466(6)$ & $0.0614(7)$ & $-0.0008(5)$ & 0.0157 (6) & $0.0071(5)$ \\
\hline $\mathrm{C} 2$ & $0.0697(8)$ & $0.0442(6)$ & $0.0557(7)$ & $-0.0018(6)$ & $0.0148(6)$ & $-0.0039(5)$ \\
\hline $\mathrm{C} 3$ & $0.0566(7)$ & $0.0405(6)$ & $0.0496(6)$ & $0.0001(5)$ & $0.0174(5)$ & $0.0016(5)$ \\
\hline $\mathrm{C} 4$ & $0.0773(12)$ & $0.0870(13)$ & $0.0938(13)$ & $-0.0075(10)$ & $0.0428(10)$ & $0.0071(11)$ \\
\hline $\mathrm{C} 5$ & $0.0621(8)$ & $0.0579(8)$ & $0.0451(6)$ & $0.0046(6)$ & $0.0070(6)$ & $-0.0045(5)$ \\
\hline C6 & $0.0568(7)$ & $0.0531(7)$ & $0.0638(8)$ & $-0.0014(6)$ & $0.0072(6)$ & $-0.0134(6)$ \\
\hline $\mathrm{C} 7$ & $0.0714(8)$ & $0.0457(6)$ & $0.0413(6)$ & $-0.0026(6)$ & $0.0116(5)$ & $-0.0068(5)$ \\
\hline $\mathrm{O} 1^{\prime}$ & $0.0769(7)$ & $0.0870(8)$ & $0.0883(8)$ & $-0.0321(6)$ & $0.0302(6)$ & $-0.0276(6)$ \\
\hline $\mathrm{O} 2^{\prime}$ & $0.0726(7)$ & $0.0773(7)$ & $0.0948(8)$ & $-0.0190(6)$ & $0.0387(6)$ & $-0.0027(6)$ \\
\hline O3' & $0.0817(8)$ & $0.0850(8)$ & $0.0804(7)$ & $-0.0055(6)$ & $0.0414(6)$ & $-0.0145(6)$ \\
\hline $\mathrm{C} 7^{\prime}$ & $0.0577(7)$ & $0.0500(7)$ & $0.0723(9)$ & $-0.0018(6)$ & $0.0211(6)$ & $0.0011(6)$ \\
\hline $\mathrm{C} 1^{\prime}$ & $0.0501(6)$ & $0.0414(6)$ & $0.0664(8)$ & $0.0038(5)$ & $0.0177(6)$ & $0.0003(5)$ \\
\hline $\mathrm{C} 2^{\prime}$ & $0.0573(7)$ & $0.0489(7)$ & $0.0672(8)$ & $0.0090(5)$ & $0.0224(6)$ & $-0.0005(6)$ \\
\hline $\mathrm{C} 3^{\prime}$ & $0.0641(8)$ & $0.0579(8)$ & $0.0750(10)$ & $0.0052(6)$ & $0.0136(7)$ & $-0.0139(7)$ \\
\hline $\mathrm{C} 4^{\prime}$ & $0.0542(8)$ & $0.0570(8)$ & $0.0942(11)$ & $-0.0026(6)$ & $0.0150(7)$ & $-0.0108(8)$ \\
\hline $\mathrm{C}^{\prime}$ & $0.0552(8)$ & $0.0628(9)$ & $0.0882(11)$ & $-0.0045(6)$ & $0.0284(7)$ & $-0.0056(7)$ \\
\hline $\mathrm{C} 6^{\prime}$ & $0.0575(7)$ & $0.0537(7)$ & $0.0728(9)$ & $-0.0007(6)$ & $0.0250(6)$ & $-0.0065(6)$ \\
\hline $\mathrm{C} 9$ & $0.0675(8)$ & $0.0617(8)$ & $0.0529(7)$ & $0.0029(7)$ & $0.0076(6)$ & $-0.0094(6)$ \\
\hline $\mathrm{C} 10$ & $0.0697(10)$ & $0.0947(13)$ & $0.0667(9)$ & $0.0059(9)$ & $0.0041(8)$ & $-0.0144(8)$ \\
\hline $\mathrm{C} 11$ & $0.0814(12)$ & $0.1051(15)$ & $0.0744(11)$ & $0.0296(11)$ & $-0.0021(9)$ & $-0.0137(10)$ \\
\hline $\mathrm{C} 12$ & $0.1147(16)$ & $0.0723(11)$ & $0.0852(12)$ & $0.0411(11)$ & $0.0056(11)$ & $-0.0024(9)$ \\
\hline $\mathrm{C} 13$ & 0.0964 (12) & $0.0533(8)$ & $0.0661(9)$ & $0.0106(8)$ & $0.0089(8)$ & $-0.0010(7)$ \\
\hline C8 & $0.0739(8)$ & $0.0508(7)$ & $0.0363(6)$ & $0.0074(6)$ & 0.0077 (5) & $-0.0034(5)$ \\
\hline
\end{tabular}


Geometric parameters $\left(\AA,{ }^{\circ}\right)$

\begin{tabular}{|c|c|c|c|}
\hline $\mathrm{O} 1-\mathrm{N} 3$ & $1.2229(16)$ & $\mathrm{O} 2^{\prime}-\mathrm{C} 7^{\prime}$ & $1.2196(17)$ \\
\hline $\mathrm{O} 2-\mathrm{N} 3$ & $1.2236(16)$ & $\mathrm{O} 3^{\prime}-\mathrm{H} 3^{\prime}$ & $0.92(2)$ \\
\hline $\mathrm{O} 3-\mathrm{C} 6$ & $1.4378(17)$ & $\mathrm{O} 3^{\prime}-\mathrm{C} 2^{\prime}$ & $1.3507(17)$ \\
\hline $\mathrm{O} 3-\mathrm{C} 7$ & $1.3441(15)$ & $\mathrm{C} 7^{\prime}-\mathrm{C} 1^{\prime}$ & $1.4704(19)$ \\
\hline $\mathrm{O} 4-\mathrm{C} 7$ & $1.2015(17)$ & $\mathrm{C} 1^{\prime}-\mathrm{C} 2^{\prime}$ & $1.3950(19)$ \\
\hline $\mathrm{N} 1-\mathrm{C} 1$ & $1.3518(16)$ & $\mathrm{C} 1^{\prime}-\mathrm{C} 6^{\prime}$ & $1.3953(19)$ \\
\hline $\mathrm{N} 1-\mathrm{C} 3$ & $1.3803(15)$ & $\mathrm{C} 2^{\prime}-\mathrm{C} 3^{\prime}$ & $1.391(2)$ \\
\hline $\mathrm{N} 1-\mathrm{C} 5$ & $1.4667(16)$ & $\mathrm{C} 3^{\prime}-\mathrm{H} 3^{\prime} \mathrm{A}$ & $0.951(18)$ \\
\hline $\mathrm{N} 2-\mathrm{C} 1$ & $1.3281(18)$ & $\mathrm{C} 3^{\prime}-\mathrm{C} 4^{\prime}$ & $1.368(2)$ \\
\hline $\mathrm{N} 2-\mathrm{C} 2$ & $1.3509(19)$ & $\mathrm{C} 4^{\prime}-\mathrm{H} 4^{\prime}$ & $1.001(19)$ \\
\hline $\mathrm{N} 3-\mathrm{C} 3$ & $1.4116(17)$ & $\mathrm{C} 4^{\prime}-\mathrm{C} 5^{\prime}$ & $1.386(2)$ \\
\hline $\mathrm{C} 1-\mathrm{C} 4$ & $1.484(2)$ & $\mathrm{C} 5^{\prime}-\mathrm{H} 5^{\prime}$ & $0.95(2)$ \\
\hline $\mathrm{C} 2-\mathrm{H} 2$ & $0.948(17)$ & $\mathrm{C} 5^{\prime}-\mathrm{C} 6^{\prime}$ & $1.374(2)$ \\
\hline $\mathrm{C} 2-\mathrm{C} 3$ & $1.3514(18)$ & $\mathrm{C} 6^{\prime}-\mathrm{H} 6^{\prime}$ & 1.019 (17) \\
\hline $\mathrm{C} 4-\mathrm{H} 4 \mathrm{~A}$ & $0.97(3)$ & $\mathrm{C} 9-\mathrm{C} 10$ & $1.384(2)$ \\
\hline $\mathrm{C} 4-\mathrm{H} 4 \mathrm{~B}$ & $0.94(3)$ & $\mathrm{C} 9-\mathrm{C} 8$ & $1.385(2)$ \\
\hline $\mathrm{C} 4-\mathrm{H} 4 \mathrm{C}$ & $0.91(3)$ & C9- $\mathrm{H} 9$ & $0.984(17)$ \\
\hline $\mathrm{C} 5-\mathrm{H} 5 \mathrm{~A}$ & $0.969(16)$ & $\mathrm{C} 10-\mathrm{C} 11$ & $1.369(3)$ \\
\hline C5-H5B & $0.980(16)$ & $\mathrm{C} 10-\mathrm{H} 10$ & $0.98(2)$ \\
\hline $\mathrm{C} 5-\mathrm{C} 6$ & $1.503(2)$ & $\mathrm{C} 11-\mathrm{C} 12$ & $1.364(3)$ \\
\hline C6-H6A & $0.988(17)$ & $\mathrm{C} 11-\mathrm{H} 11$ & $1.02(2)$ \\
\hline C6-H6B & 0.965 (17) & $\mathrm{C} 12-\mathrm{C} 13$ & $1.386(3)$ \\
\hline $\mathrm{C} 7-\mathrm{C} 8$ & $1.478(2)$ & $\mathrm{C} 12-\mathrm{H} 12$ & $0.91(2)$ \\
\hline $\mathrm{O} 1^{\prime}-\mathrm{H} 1^{\prime}$ & $0.91(2)$ & $\mathrm{C} 13-\mathrm{C} 8$ & $1.392(2)$ \\
\hline $\mathrm{O} 1^{\prime}-\mathrm{C} 7^{\prime}$ & $1.3111(18)$ & $\mathrm{C} 13-\mathrm{H} 13$ & $0.925(18)$ \\
\hline $\mathrm{C} 7-\mathrm{O} 3-\mathrm{C} 6$ & $115.08(11)$ & $\mathrm{O} 1^{\prime}-\mathrm{C} 7^{\prime}-\mathrm{C} 1^{\prime}$ & $114.06(12)$ \\
\hline $\mathrm{C} 1-\mathrm{N} 1-\mathrm{C} 3$ & $105.29(10)$ & $\mathrm{O} 2^{\prime}-\mathrm{C}^{\prime}-\mathrm{O} 1^{\prime}$ & $122.59(14)$ \\
\hline $\mathrm{C} 1-\mathrm{N} 1-\mathrm{C} 5$ & $125.82(11)$ & $\mathrm{O} 2^{\prime}-\mathrm{C}^{\prime}-\mathrm{C} 1^{\prime}$ & $123.34(14)$ \\
\hline $\mathrm{C} 3-\mathrm{N} 1-\mathrm{C} 5$ & $128.73(11)$ & $\mathrm{C} 2^{\prime}-\mathrm{C} 1^{\prime}-\mathrm{C} 7^{\prime}$ & $120.02(12)$ \\
\hline $\mathrm{C} 1-\mathrm{N} 2-\mathrm{C} 2$ & $106.89(11)$ & $\mathrm{C} 2^{\prime}-\mathrm{C} 1^{\prime}-\mathrm{C} 6^{\prime}$ & $119.06(12)$ \\
\hline $\mathrm{O} 1-\mathrm{N} 3-\mathrm{O} 2$ & $124.02(13)$ & $\mathrm{C} 6^{\prime}-\mathrm{C} 1^{\prime}-\mathrm{C} 7^{\prime}$ & $120.90(13)$ \\
\hline $\mathrm{O} 1-\mathrm{N} 3-\mathrm{C} 3$ & $116.32(12)$ & $\mathrm{O} 3^{\prime}-\mathrm{C} 2^{\prime}-\mathrm{C} 1^{\prime}$ & $122.74(13)$ \\
\hline $\mathrm{O} 2-\mathrm{N} 3-\mathrm{C} 3$ & $119.66(12)$ & $\mathrm{O}^{\prime}-\mathrm{C} 2^{\prime}-\mathrm{C} 3^{\prime}$ & $117.89(14)$ \\
\hline $\mathrm{N} 1-\mathrm{C} 1-\mathrm{C} 4$ & $125.10(15)$ & $\mathrm{C} 3^{\prime}-\mathrm{C} 2^{\prime}-\mathrm{C} 1^{\prime}$ & $119.37(13)$ \\
\hline $\mathrm{N} 2-\mathrm{C} 1-\mathrm{N} 1$ & $111.15(11)$ & $\mathrm{C} 2^{\prime}-\mathrm{C} 3^{\prime}-\mathrm{H} 3^{\prime} \mathrm{A}$ & $118.1(11)$ \\
\hline $\mathrm{N} 2-\mathrm{C} 1-\mathrm{C} 4$ & $123.75(15)$ & $\mathrm{C} 4^{\prime}-\mathrm{C} 3^{\prime}-\mathrm{C} 2^{\prime}$ & $120.49(15)$ \\
\hline $\mathrm{N} 2-\mathrm{C} 2-\mathrm{H} 2$ & $122.0(10)$ & $\mathrm{C} 4^{\prime}-\mathrm{C} 3^{\prime}-\mathrm{H} 3^{\prime} \mathrm{A}$ & $121.4(11)$ \\
\hline $\mathrm{N} 2-\mathrm{C} 2-\mathrm{C} 3$ & $108.78(12)$ & $\mathrm{C} 3^{\prime}-\mathrm{C} 4^{\prime}-\mathrm{H} 4^{\prime}$ & $118.4(11)$ \\
\hline $\mathrm{C} 3-\mathrm{C} 2-\mathrm{H} 2$ & $129.2(10)$ & $\mathrm{C} 3^{\prime}-\mathrm{C} 4^{\prime}-\mathrm{C} 5^{\prime}$ & $120.82(14)$ \\
\hline $\mathrm{N} 1-\mathrm{C} 3-\mathrm{N} 3$ & $125.27(11)$ & $\mathrm{C} 5^{\prime}-\mathrm{C} 4^{\prime}-\mathrm{H} 4^{\prime}$ & $120.7(11)$ \\
\hline $\mathrm{C} 2-\mathrm{C} 3-\mathrm{N} 1$ & $107.88(11)$ & $\mathrm{C} 4^{\prime}-\mathrm{C} 5^{\prime}-\mathrm{H} 5^{\prime}$ & $120.2(12)$ \\
\hline $\mathrm{C} 2-\mathrm{C} 3-\mathrm{N} 3$ & $126.83(12)$ & $\mathrm{C} 6^{\prime}-\mathrm{C} 5^{\prime}-\mathrm{C} 4^{\prime}$ & $119.11(15)$ \\
\hline $\mathrm{C} 1-\mathrm{C} 4-\mathrm{H} 4 \mathrm{~A}$ & $113.1(19)$ & $\mathrm{C} 6^{\prime}-\mathrm{C} 5^{\prime}-\mathrm{H} 5^{\prime}$ & $120.7(12)$ \\
\hline $\mathrm{C} 1-\mathrm{C} 4-\mathrm{H} 4 \mathrm{~B}$ & $113.2(17)$ & $\mathrm{C} 1^{\prime}-\mathrm{C} 6^{\prime}-\mathrm{H} 6^{\prime}$ & $118.1(9)$ \\
\hline $\mathrm{C} 1-\mathrm{C} 4-\mathrm{H} 4 \mathrm{C}$ & 107.7 (19) & $\mathrm{C} 5^{\prime}-\mathrm{C} 6^{\prime}-\mathrm{C} 1^{\prime}$ & $121.13(14)$ \\
\hline
\end{tabular}




$\begin{array}{llll}\mathrm{H} 4 \mathrm{~A}-\mathrm{C} 4-\mathrm{H} 4 \mathrm{~B} & 102(2) & \mathrm{C} 5^{\prime}-\mathrm{C} 6{ }^{\prime}-\mathrm{H} 6^{\prime} & 120.8(9) \\ \mathrm{H} 4 \mathrm{~A}-\mathrm{C} 4-\mathrm{H} 4 \mathrm{C} & 105(2) & \mathrm{C} 10-\mathrm{C} 9-\mathrm{C} 8 & 120.28(15) \\ \mathrm{H} 4 \mathrm{~B}-\mathrm{C} 4-\mathrm{H} 4 \mathrm{C} & 116(3) & \mathrm{C} 10-\mathrm{C} 9-\mathrm{H} 9 & 120.7(10) \\ \mathrm{N} 1-\mathrm{C} 5-\mathrm{H} 5 \mathrm{~A} & 106.3(9) & \mathrm{C} 8-\mathrm{C} 9-\mathrm{H} 9 & 119.0(10) \\ \mathrm{N} 1-\mathrm{C} 5-\mathrm{H} 5 \mathrm{~B} & 106.4(9) & \mathrm{C} 9-\mathrm{C} 10-\mathrm{H} 10 & 121.0(14) \\ \mathrm{N} 1-\mathrm{C} 5-\mathrm{C} 6 & 113.88(11) & \mathrm{C} 11-\mathrm{C} 10-\mathrm{C} 9 & 120.15(19) \\ \mathrm{H} 5 \mathrm{~A}-\mathrm{C} 5-\mathrm{H} 5 \mathrm{~B} & 109.7(13) & \mathrm{C} 11-\mathrm{C} 10-\mathrm{H} 10 & 118.8(14) \\ \mathrm{C} 6-\mathrm{C} 5-\mathrm{H} 5 \mathrm{~A} & 109.7(9) & \mathrm{C} 10-\mathrm{C} 11-\mathrm{H} 11 & 119.4(13) \\ \mathrm{C} 6-\mathrm{C} 5-\mathrm{H} 5 \mathrm{~B} & 110.6(9) & \mathrm{C} 12-\mathrm{C} 11-\mathrm{C} 10 & 120.21(19) \\ \mathrm{O} 3-\mathrm{C} 6-\mathrm{C} 5 & 107.94(12) & \mathrm{C} 12-\mathrm{C} 11-\mathrm{H} 11 & 120.3(13) \\ \mathrm{O} 3-\mathrm{C} 6-\mathrm{H} 6 \mathrm{~A} & 109.8(10) & \mathrm{C} 11-\mathrm{C} 12-\mathrm{C} 13 & 120.64(18) \\ \mathrm{O} 3-\mathrm{C} 6-\mathrm{H} 6 \mathrm{~B} & 109.6(10) & \mathrm{C} 11-\mathrm{C} 12-\mathrm{H} 12 & 122.2(17) \\ \mathrm{C} 5-\mathrm{C} 6-\mathrm{H} 6 \mathrm{~A} & 111.0(9) & \mathrm{C} 13-\mathrm{C} 12-\mathrm{H} 12 & 117.1(17) \\ \mathrm{C} 5-\mathrm{C} 6-\mathrm{H} 6 \mathrm{~B} & 109.3(10) & \mathrm{C} 12-\mathrm{C} 13-\mathrm{C} 8 & 122.8(19) \\ \mathrm{H} 6 \mathrm{~A}-\mathrm{C} 6-\mathrm{H} 6 \mathrm{~B} & 109.1(13) & \mathrm{C} 12-\mathrm{C} 13-\mathrm{H} 13 & 117.5(11) \\ \mathrm{O} 3-\mathrm{C} 7-\mathrm{C} 8 & 112.36(11) & \mathrm{C} 8-\mathrm{C} 13-\mathrm{H} 13 & 122.78(12) \\ \mathrm{O} 4-\mathrm{C} 7-\mathrm{O} 3 & 122.24(13) & \mathrm{C} 9-\mathrm{C} 8-\mathrm{C} 7 & 119.05(15) \\ \mathrm{O} 4-\mathrm{C} 7-\mathrm{C} 8 & 125.38(12) & \mathrm{C} 9-\mathrm{C} 8-\mathrm{C} 13 & 118.15(14) \\ \mathrm{C} 77^{\prime}-\mathrm{O} 1^{\prime}-\mathrm{H} 1^{\prime} & 111.8(14) & \mathrm{C} 13-\mathrm{C} 8-\mathrm{C} 7 & \\ \mathrm{C} 2^{\prime}-\mathrm{O} 3-\mathrm{H} 3^{\prime} & 106.2(14) & & \end{array}$

1-[2-(Benzoyloxy)ethyl]-2-methyl-5-nitro-1 H-imidazol-3-ium 3-carboxyprop-2-enoate (bzmdmlc)

\section{Crystal data}

$\mathrm{C}_{13} \mathrm{H}_{14} \mathrm{~N}_{3} \mathrm{O}_{4}^{+} \cdot \mathrm{C}_{4} \mathrm{H}_{3} \mathrm{O}_{4}^{-}$

$M_{r}=391.33$

Monoclinic, $C 2 / c$

$a=43.130(3) \AA$

$b=5.7944(4) \AA$

$c=15.2645(11) \AA$

$\beta=109.600(3)^{\circ}$

$V=3593.7(5) \AA^{3}$

$Z=8$

\section{Data collection}

Bruker D8 VENTURE Kappa Duo PHOTON II CPAD diffractometer

Multilayer mirrors monochromator $\varphi$ and $\omega$ scans

Absorption correction: multi-scan

(SADABS; Bruker, 2016)

$T_{\min }=0.261, T_{\max }=0.746$

\section{Refinement}

Refinement on $F^{2}$

Least-squares matrix: full

$R\left[F^{2}>2 \sigma\left(F^{2}\right)\right]=0.056$

$w R\left(F^{2}\right)=0.147$

$S=1.02$

4130 reflections

322 parameters
$F(000)=1632$

$D_{\mathrm{x}}=1.447 \mathrm{Mg} \mathrm{m}^{-3}$

Mo $K \alpha$ radiation, $\lambda=0.71073 \AA$

Cell parameters from 6422 reflections

$\theta=2.7-26.1^{\circ}$

$\mu=0.12 \mathrm{~mm}^{-1}$

$T=302 \mathrm{~K}$

Prism, clear light colourless

$0.57 \times 0.23 \times 0.05 \mathrm{~mm}$

20803 measured reflections

4130 independent reflections

2159 reflections with $I>2 \sigma(I)$

$R_{\text {int }}=0.089$

$\theta_{\max }=27.5^{\circ}, \theta_{\min }=2.7^{\circ}$

$h=-56 \rightarrow 49$

$k=-7 \rightarrow 7$

$l=-19 \rightarrow 19$

0 restraints

Hydrogen site location: difference Fourier map

All $\mathrm{H}$-atom parameters refined

$w=1 /\left[\sigma^{2}\left(F_{\mathrm{o}}^{2}\right)+(0.0536 P)^{2}+0.8177 P\right]$

where $P=\left(F_{\mathrm{o}}{ }^{2}+2 F_{\mathrm{c}}{ }^{2}\right) / 3$

$(\Delta / \sigma)_{\max }<0.001$

$\Delta \rho_{\max }=0.18$ e $\AA^{-3}$ 
$\Delta \rho_{\min }=-0.21$ e $\AA^{-3}$
Extinction correction: SHELXL2018

(Sheldrick, 2015b),

$\mathrm{Fc}^{*}=\mathrm{kFc}\left[1+0.001 \mathrm{xFc}^{2} \lambda^{3} / \sin (2 \theta)\right]^{-1 / 4}$

Extinction coefficient: 0.0015 (3)

\section{Special details}

Geometry. All esds (except the esd in the dihedral angle between two 1.s. planes) are estimated using the full covariance matrix. The cell esds are taken into account individually in the estimation of esds in distances, angles and torsion angles; correlations between esds in cell parameters are only used when they are defined by crystal symmetry. An approximate (isotropic) treatment of cell esds is used for estimating esds involving l.s. planes.

Refinement. Single-crystal X-ray diffraction data collection ( scans and $\omega$ scans with $\kappa$ and $\theta$ offsets) were performed at room temperature on a Bruker AXS D8 VENTURE equipped with a Kappa goniometer, PHOTON II CPAD detector, a Mo $K \alpha$ INCOATEC I $\mu$ S 3.0 microfocus source $(\lambda=0.71073 \AA)$. The crystal centring, unit-cell determination, refinement of the unit-cell parameters and data collection was controlled through the program APEX3 (Bruker, 2012). The frame integration was performed using SAINT (Bruker, 2016) and the intensities were scaled and absorption corrected using SADABS (Bruker, 2001). Using OLEX2 (Dolomanov et al., 2009), the structure was solved by intrinsic phasing using SHELXT (Sheldrick, 2015) and refined by full-matrix least-squares calculation based on $F^{2}$ for all reflection using SHELXL (Sheldrick, 2007). All non-H atoms were refined anisotropically.

Fractional atomic coordinates and isotropic or equivalent isotropic displacement parameters $\left(\hat{A}^{2}\right)$

\begin{tabular}{|c|c|c|c|c|}
\hline & $x$ & $y$ & $z$ & $U_{\text {iso }} * / U_{\text {eq }}$ \\
\hline $\mathrm{O} 3$ & $0.68004(4)$ & $0.6877(3)$ & $0.23662(13)$ & $0.0507(5)$ \\
\hline $\mathrm{O} 1^{\prime}$ & $0.55288(4)$ & $-0.0276(3)$ & $0.21210(14)$ & $0.0618(6)$ \\
\hline O4 & $0.66174(4)$ & $0.3229(3)$ & $0.22612(14)$ & $0.0603(5)$ \\
\hline N1 & $0.61358(4)$ & $0.6620(3)$ & $0.25880(13)$ & $0.0390(5)$ \\
\hline N2 & $0.58882(5)$ & $0.3456(4)$ & $0.27408(15)$ & $0.0478(5)$ \\
\hline $\mathrm{O}^{\prime}$ & $0.45356(6)$ & 0.2178 & $0.08155(16)$ & $0.0761(6)$ \\
\hline $\mathrm{O} 2^{\prime}$ & $0.51306(5)$ & $0.2206(3)$ & $0.15210(17)$ & $0.0820(7)$ \\
\hline $\mathrm{O} 4^{\prime}$ & $0.41448(5)$ & $-0.0382(4)$ & $0.03379(16)$ & $0.0823(7)$ \\
\hline $\mathrm{O} 2$ & $0.65266(6)$ & $0.9311(4)$ & $0.41184(14)$ & $0.0826(7)$ \\
\hline N3 & $0.64430(5)$ & $0.7380(4)$ & $0.42711(17)$ & $0.0569(6)$ \\
\hline O1 & $0.65247(6)$ & $0.6494(4)$ & $0.50428(14)$ & $0.0831(7)$ \\
\hline $\mathrm{C} 3$ & $0.62288(6)$ & $0.6050(4)$ & $0.35213(17)$ & $0.0421(6)$ \\
\hline $\mathrm{C} 1$ & $0.59273(6)$ & $0.4969(4)$ & $0.21254(17)$ & $0.0424(6)$ \\
\hline $\mathrm{C} 8$ & $0.71780(6)$ & $0.4014(4)$ & $0.31383(17)$ & $0.0434(6)$ \\
\hline $\mathrm{C} 7$ & $0.68393(6)$ & $0.4588(4)$ & $0.25489(18)$ & $0.0444(6)$ \\
\hline $\mathrm{C} 5$ & $0.62531(7)$ & $0.8476(4)$ & $0.2115(2)$ & $0.0442(6)$ \\
\hline $\mathrm{C} 2$ & $0.60735(6)$ & $0.4094(4)$ & $0.3611(2)$ & $0.0491(7)$ \\
\hline C6 & $0.64965(7)$ & $0.7620(5)$ & $0.1677(2)$ & $0.0495(7)$ \\
\hline C13 & $0.74353(7)$ & $0.5571(5)$ & $0.3278(2)$ & $0.0499(7)$ \\
\hline $\mathrm{C} 1^{\prime}$ & $0.52336(7)$ & 0.0167 (4) & $0.1699(2)$ & $0.0511(7)$ \\
\hline $\mathrm{C} 2^{\prime}$ & $0.50025(7)$ & $-0.1817(4)$ & $0.1411(2)$ & $0.0532(7)$ \\
\hline C9 & $0.72408(7)$ & $0.1829(4)$ & 0.35499 (19) & $0.0507(7)$ \\
\hline $\mathrm{C} 4^{\prime}$ & $0.44353(7)$ & $0.0053(5)$ & 0.07005 (19) & $0.0556(7)$ \\
\hline C3' & $0.46770(7)$ & $-0.1857(5)$ & $0.1014(2)$ & $0.0543(7)$ \\
\hline $\mathrm{C} 12$ & $0.77498(7)$ & $0.4969(5)$ & $0.3810(2)$ & $0.0568(7)$ \\
\hline $\mathrm{C} 11$ & $0.78124(8)$ & $0.2810(5)$ & $0.4226(2)$ & $0.0587(8)$ \\
\hline $\mathrm{C} 10$ & $0.75578(8)$ & $0.1264(5)$ & $0.4091(2)$ & $0.0585(8)$ \\
\hline $\mathrm{C} 4$ & $0.57704(9)$ & $0.4779(6)$ & $0.1113(2)$ & $0.0587(8)$ \\
\hline
\end{tabular}




$\begin{array}{lllll}\text { H5A } & 0.6059(6) & 0.907(4) & 0.1597(16) & 0.045(7)^{*} \\ \text { H13 } & 0.7388(6) & 0.707(4) & 0.2973(16) & 0.052(7)^{*} \\ \text { H5B } & 0.6357(5) & 0.968(4) & 0.2571(16) & 0.043(7)^{*} \\ \text { H6A } & 0.6413(6) & 0.631(5) & 0.1212(18) & 0.063(8)^{*} \\ \text { H2' } & 0.5118(6) & -0.330(4) & 0.1577(16) & 0.055(7)^{*} \\ \text { H2A } & 0.6072(6) & 0.328(4) & 0.4128(17) & 0.048(7)^{*} \\ \text { H12 } & 0.7929(7) & 0.609(5) & 0.3919(18) & 0.070(9)^{*} \\ \text { H6B } & 0.6568(6) & 0.893(5) & 0.1377(19) & 0.067(8)^{*} \\ \text { H10 } & 0.7590(6) & -0.031(5) & 0.4418(19) & 0.073(9)^{*} \\ \text { H4A } & 0.5560(9) & 0.388(6) & 0.096(2) & 0.107(12)^{*} \\ \text { H4B } & 0.5743(8) & 0.625(6) & 0.082(2) & 0.093(11)^{*} \\ \text { H9 } & 0.7040(7) & 0.076(5) & 0.3437(19) & 0.077(9)^{*} \\ \text { H3'A } & 0.4572(7) & -0.335(5) & 0.0881(19) & 0.073(9)^{*} \\ \text { H2 } & 0.5745(8) & 0.206(5) & 0.255(2) & 0.089(10)^{*} \\ \text { H11 } & 0.8042(7) & 0.243(5) & 0.4636(19) & 0.074(9)^{*} \\ \text { H4C } & 0.5910(8) & 0.392(6) & 0.085(2) & 0.107(13)^{*} \\ \text { H3' } & 0.4806(11) & 0.222(6) & 0.104(3) & 0.125(13)^{*}\end{array}$

Atomic displacement parameters $\left(\AA^{2}\right)$

\begin{tabular}{|c|c|c|c|c|c|c|}
\hline & $U^{11}$ & $U^{22}$ & $U^{33}$ & $U^{12}$ & $U^{13}$ & $U^{23}$ \\
\hline $\mathrm{O} 3$ & $0.0424(10)$ & $0.0375(9)$ & $0.0727(12)$ & $-0.0004(8)$ & $0.0200(9)$ & $0.0034(8)$ \\
\hline $\mathrm{O} 1^{\prime}$ & $0.0417(11)$ & $0.0478(11)$ & $0.0899(15)$ & $-0.0047(9)$ & $0.0142(10)$ & $-0.0070(10)$ \\
\hline $\mathrm{O} 4$ & $0.0523(11)$ & $0.0405(10)$ & $0.0828(14)$ & $-0.0081(9)$ & $0.0158(10)$ & $-0.0015(9)$ \\
\hline N1 & $0.0381(11)$ & $0.0325(10)$ & $0.0461(12)$ & $-0.0026(9)$ & $0.0136(10)$ & $-0.0003(9)$ \\
\hline $\mathrm{N} 2$ & $0.0472(13)$ & $0.0411(12)$ & $0.0550(14)$ & $-0.0090(10)$ & $0.0171(11)$ & $-0.0029(11)$ \\
\hline O3' & $0.0631(14)$ & $0.0454(12)$ & $0.1100(18)$ & $0.0084(10)$ & $0.0160(13)$ & $0.0107(11)$ \\
\hline $\mathrm{O} 2^{\prime}$ & $0.0565(13)$ & $0.0390(11)$ & $0.140(2)$ & $-0.0027(10)$ & $0.0187(14)$ & $0.0045(12)$ \\
\hline $\mathrm{O}^{\prime}$ & $0.0483(13)$ & $0.0800(15)$ & $0.1015(17)$ & $0.0036(11)$ & $0.0027(12)$ & $-0.0202(13)$ \\
\hline $\mathrm{O} 2$ & 0.0995 (17) & $0.0666(14)$ & $0.0724(15)$ & $-0.0391(13)$ & $0.0165(13)$ & $-0.0087(11)$ \\
\hline N3 & $0.0533(14)$ & $0.0563(14)$ & $0.0560(16)$ & $-0.0119(12)$ & $0.0115(12)$ & $-0.0057(12)$ \\
\hline $\mathrm{O} 1$ & $0.0933(17)$ & $0.0892(16)$ & $0.0482(13)$ & $-0.0182(13)$ & $-0.0010(12)$ & $0.0089(12)$ \\
\hline $\mathrm{C} 3$ & $0.0391(14)$ & $0.0414(13)$ & $0.0442(15)$ & $-0.0025(11)$ & $0.0120(12)$ & $-0.0001(11)$ \\
\hline $\mathrm{C} 1$ & $0.0382(13)$ & $0.0389(13)$ & $0.0495(16)$ & $-0.0009(11)$ & $0.0139(12)$ & $-0.0009(12)$ \\
\hline $\mathrm{C} 8$ & $0.0455(15)$ & $0.0389(13)$ & $0.0502(15)$ & $-0.0023(11)$ & $0.0217(13)$ & $-0.0074(11)$ \\
\hline $\mathrm{C} 7$ & $0.0493(16)$ & $0.0345(13)$ & $0.0566(16)$ & $-0.0036(12)$ & $0.0273(13)$ & $-0.0049(12)$ \\
\hline $\mathrm{C} 5$ & $0.0449(15)$ & $0.0337(13)$ & $0.0541(17)$ & $-0.0012(12)$ & $0.0169(14)$ & $0.0069(13)$ \\
\hline $\mathrm{C} 2$ & $0.0500(16)$ & $0.0452(15)$ & $0.0514(18)$ & $-0.0066(12)$ & $0.0163(14)$ & $0.0037(14)$ \\
\hline C6 & $0.0489(16)$ & $0.0453(15)$ & $0.0570(17)$ & $-0.0004(13)$ & $0.0215(14)$ & 0.0077 (14) \\
\hline $\mathrm{C} 13$ & $0.0472(16)$ & $0.0434(15)$ & $0.0611(18)$ & $-0.0022(13)$ & $0.0208(14)$ & $-0.0014(13)$ \\
\hline $\mathrm{C} 1^{\prime}$ & $0.0471(16)$ & $0.0410(15)$ & $0.0672(19)$ & $-0.0036(13)$ & $0.0221(14)$ & $-0.0058(13)$ \\
\hline $\mathrm{C} 2^{\prime}$ & $0.0520(17)$ & $0.0337(13)$ & $0.0702(19)$ & -0.0014 & $0.0157(15)$ & $-0.0083(13)$ \\
\hline $\mathrm{C} 9$ & $0.0582(18)$ & $0.0403(14)$ & $0.0547(17)$ & $-0.0007(14)$ & $0.0205(15)$ & $-0.0046(12)$ \\
\hline $\mathrm{C} 4^{\prime}$ & $0.0481(17)$ & $0.0578(17)$ & $0.0555(18)$ & 0.0052 (14) & $0.0101(14)$ & $-0.0062(14)$ \\
\hline $\mathrm{C} 3^{\prime}$ & $0.0491(17)$ & $0.0397(15)$ & $0.0679(19)$ & $-0.0043(13)$ & $0.0113(14)$ & $-0.0115(13)$ \\
\hline $\mathrm{C} 12$ & $0.0500(17)$ & $0.0546(17)$ & $0.068(2)$ & $-0.0046(15)$ & $0.0224(15)$ & $-0.0073(15)$ \\
\hline C11 & $0.0530(18)$ & $0.0646(19)$ & $0.0543(18)$ & $0.0110(16)$ & $0.0122(15)$ & $-0.0082(15)$ \\
\hline $\mathrm{C} 10$ & $0.071(2)$ & $0.0482(16)$ & $0.0541(18)$ & $0.0090(16)$ & $0.0189(16)$ & $-0.0020(14)$ \\
\hline
\end{tabular}




$\begin{array}{lllllll}\mathrm{C} 4 & 0.062(2) & 0.0612(19) & 0.0483(18) & -0.0114(17) & 0.0126(16) & -0.0067(16)\end{array}$

Geometric parameters $(\AA, \stackrel{o}{)}$

\begin{tabular}{|c|c|c|c|}
\hline $\mathrm{O} 3-\mathrm{C} 7$ & $1.354(3)$ & $\mathrm{C} 5-\mathrm{C} 6$ & $1.505(4)$ \\
\hline $\mathrm{O} 3-\mathrm{C} 6$ & $1.444(3)$ & $\mathrm{C} 5-\mathrm{H} 5 \mathrm{~A}$ & $1.00(2)$ \\
\hline $\mathrm{O} 1^{\prime}-\mathrm{C} 1^{\prime}$ & $1.246(3)$ & $\mathrm{C} 5-\mathrm{H} 5 \mathrm{~B}$ & $0.98(2)$ \\
\hline $\mathrm{O} 4-\mathrm{C} 7$ & $1.202(3)$ & $\mathrm{C} 2-\mathrm{H} 2 \mathrm{~A}$ & $0.92(2)$ \\
\hline $\mathrm{N} 1-\mathrm{C} 3$ & $1.384(3)$ & $\mathrm{C} 6-\mathrm{H} 6 \mathrm{~A}$ & $1.02(3)$ \\
\hline $\mathrm{N} 1-\mathrm{C} 1$ & $1.340(3)$ & $\mathrm{C} 6-\mathrm{H} 6 \mathrm{~B}$ & $0.99(3)$ \\
\hline $\mathrm{N} 1-\mathrm{C} 5$ & $1.476(3)$ & $\mathrm{C} 13-\mathrm{C} 12$ & $1.372(4)$ \\
\hline $\mathrm{N} 2-\mathrm{C} 1$ & $1.336(3)$ & $\mathrm{C} 13-\mathrm{H} 13$ & $0.97(2)$ \\
\hline $\mathrm{N} 2-\mathrm{C} 2$ & $1.351(3)$ & $\mathrm{C} 1^{\prime}-\mathrm{C} 2^{\prime}$ & $1.488(3)$ \\
\hline $\mathrm{N} 2-\mathrm{H} 2$ & $1.00(3)$ & $\mathrm{C} 2^{\prime}-\mathrm{C} 3^{\prime}$ & $1.330(4)$ \\
\hline $\mathrm{O} 3^{\prime}-\mathrm{C} 4^{\prime}$ & $1.297(3)$ & $\mathrm{C} 2^{\prime}-\mathrm{H} 2^{\prime}$ & $0.98(2)$ \\
\hline $\mathrm{O} 3^{\prime}-\mathrm{H} 3^{\prime}$ & $1.10(4)$ & $\mathrm{C} 9-\mathrm{C} 10$ & $1.379(4)$ \\
\hline $\mathrm{O} 2^{\prime}-\mathrm{Cl}^{\prime}$ & $1.259(3)$ & $\mathrm{C} 9-\mathrm{H} 9$ & $1.03(3)$ \\
\hline $\mathrm{O} 2^{\prime}-\mathrm{H} 3^{\prime}$ & $1.35(4)$ & $\mathrm{C} 4^{\prime}-\mathrm{C} 3^{\prime}$ & $1.485(4)$ \\
\hline $\mathrm{O} 4^{\prime}-\mathrm{C} 4^{\prime}$ & $1.214(3)$ & $\mathrm{C} 3^{\prime}-\mathrm{H} 3^{\prime} \mathrm{A}$ & $0.97(3)$ \\
\hline $\mathrm{O} 2-\mathrm{N} 3$ & $1.222(3)$ & $\mathrm{C} 12-\mathrm{C} 11$ & $1.388(4)$ \\
\hline $\mathrm{N} 3-\mathrm{O} 1$ & $1.223(3)$ & $\mathrm{C} 12-\mathrm{H} 12$ & $0.98(3)$ \\
\hline $\mathrm{N} 3-\mathrm{C} 3$ & $1.431(3)$ & $\mathrm{C} 11-\mathrm{C} 10$ & $1.378(4)$ \\
\hline $\mathrm{C} 3-\mathrm{C} 2$ & $1.347(3)$ & $\mathrm{C} 11-\mathrm{H} 11$ & $1.00(3)$ \\
\hline $\mathrm{C} 1-\mathrm{C} 4$ & $1.469(4)$ & $\mathrm{C} 10-\mathrm{H} 10$ & $1.02(3)$ \\
\hline $\mathrm{C} 8-\mathrm{C} 7$ & $1.474(3)$ & $\mathrm{C} 4-\mathrm{H} 4 \mathrm{~A}$ & $1.00(4)$ \\
\hline $\mathrm{C} 8-\mathrm{C} 13$ & $1.390(3)$ & $\mathrm{C} 4-\mathrm{H} 4 \mathrm{~B}$ & $0.95(3)$ \\
\hline $\mathrm{C} 8-\mathrm{C} 9$ & $1.399(3)$ & $\mathrm{C} 4-\mathrm{H} 4 \mathrm{C}$ & $0.96(3)$ \\
\hline $\mathrm{C} 7-\mathrm{O} 3-\mathrm{C} 6$ & $117.5(2)$ & $\mathrm{C} 5-\mathrm{C} 6-\mathrm{H} 6 \mathrm{~A}$ & $114.8(15)$ \\
\hline $\mathrm{C} 3-\mathrm{N} 1-\mathrm{C} 5$ & $130.4(2)$ & $\mathrm{C} 5-\mathrm{C} 6-\mathrm{H} 6 \mathrm{~B}$ & $109.1(15)$ \\
\hline $\mathrm{C} 1-\mathrm{N} 1-\mathrm{C} 3$ & $106.48(19)$ & $\mathrm{H} 6 \mathrm{~A}-\mathrm{C} 6-\mathrm{H} 6 \mathrm{~B}$ & $110(2)$ \\
\hline $\mathrm{C} 1-\mathrm{N} 1-\mathrm{C} 5$ & $122.8(2)$ & $\mathrm{C} 8-\mathrm{C} 13-\mathrm{H} 13$ & $118.5(14)$ \\
\hline $\mathrm{C} 1-\mathrm{N} 2-\mathrm{C} 2$ & $109.8(2)$ & $\mathrm{C} 12-\mathrm{C} 13-\mathrm{C} 8$ & $120.4(3)$ \\
\hline $\mathrm{C} 1-\mathrm{N} 2-\mathrm{H} 2$ & $122.4(17)$ & $\mathrm{C} 12-\mathrm{C} 13-\mathrm{H} 13$ & $121.0(15)$ \\
\hline $\mathrm{C} 2-\mathrm{N} 2-\mathrm{H} 2$ & $127.8(17)$ & $\mathrm{O} 1^{\prime}-\mathrm{C} 1^{\prime}-\mathrm{O} 2^{\prime}$ & $122.0(2)$ \\
\hline $\mathrm{C} 4^{\prime}-\mathrm{O} 3^{\prime}-\mathrm{H} 3^{\prime}$ & $109.5(19)$ & $\mathrm{O} 1^{\prime}-\mathrm{C} 1^{\prime}-\mathrm{C} 2^{\prime}$ & $117.4(2)$ \\
\hline $\mathrm{C} 1^{\prime}-\mathrm{O} 2^{\prime}-\mathrm{H} 3^{\prime}$ & $110.5(16)$ & $\mathrm{O} 2^{\prime}-\mathrm{C} 1^{\prime}-\mathrm{C} 2^{\prime}$ & $120.6(2)$ \\
\hline $\mathrm{O} 2-\mathrm{N} 3-\mathrm{O} 1$ & $124.0(2)$ & $\mathrm{C} 1^{\prime}-\mathrm{C} 2^{\prime}-\mathrm{H} 2^{\prime}$ & $111.7(14)$ \\
\hline $\mathrm{O} 2-\mathrm{N} 3-\mathrm{C} 3$ & $119.5(2)$ & $\mathrm{C} 3^{\prime}-\mathrm{C} 2^{\prime}-\mathrm{C} 1^{\prime}$ & $130.4(3)$ \\
\hline $\mathrm{O} 1-\mathrm{N} 3-\mathrm{C} 3$ & $116.4(2)$ & $\mathrm{C} 3^{\prime}-\mathrm{C} 2^{\prime}-\mathrm{H} 2^{\prime}$ & $117.9(14)$ \\
\hline $\mathrm{N} 1-\mathrm{C} 3-\mathrm{N} 3$ & $125.5(2)$ & $\mathrm{C} 8-\mathrm{C} 9-\mathrm{H} 9$ & $116.3(15)$ \\
\hline $\mathrm{C} 2-\mathrm{C} 3-\mathrm{N} 1$ & $108.8(2)$ & $\mathrm{C} 10-\mathrm{C} 9-\mathrm{C} 8$ & $119.2(3)$ \\
\hline $\mathrm{C} 2-\mathrm{C} 3-\mathrm{N} 3$ & $125.6(2)$ & $\mathrm{C} 10-\mathrm{C} 9-\mathrm{H} 9$ & $124.5(15)$ \\
\hline $\mathrm{N} 1-\mathrm{C} 1-\mathrm{C} 4$ & $126.8(2)$ & $\mathrm{O}^{\prime}-\mathrm{C} 4^{\prime}-\mathrm{C} 3^{\prime}$ & $119.8(2)$ \\
\hline $\mathrm{N} 2-\mathrm{C} 1-\mathrm{N} 1$ & $108.6(2)$ & $\mathrm{O} 4^{\prime}-\mathrm{C} 4^{\prime}-\mathrm{O}^{\prime}$ & $120.3(3)$ \\
\hline $\mathrm{N} 2-\mathrm{C} 1-\mathrm{C} 4$ & $124.6(2)$ & $\mathrm{O} 4^{\prime}-\mathrm{C}^{\prime}-\mathrm{C} 3^{\prime}$ & $119.8(3)$ \\
\hline $\mathrm{C} 13-\mathrm{C} 8-\mathrm{C} 7$ & $121.6(2)$ & $\mathrm{C} 2^{\prime}-\mathrm{C} 3^{\prime}-\mathrm{C} 4^{\prime}$ & $130.8(3)$ \\
\hline $\mathrm{C} 13-\mathrm{C} 8-\mathrm{C} 9$ & $119.6(3)$ & $\mathrm{C} 2^{\prime}-\mathrm{C} 3^{\prime}-\mathrm{H} 3^{\prime} \mathrm{A}$ & $117.3(17)$ \\
\hline
\end{tabular}




$\begin{array}{llll}\mathrm{C} 9-\mathrm{C} 8-\mathrm{C} 7 & 118.8(2) & \mathrm{C}^{\prime}-\mathrm{C} 3^{\prime}-\mathrm{H}^{\prime} \mathrm{A} & 111.9(17) \\ \mathrm{O} 3-\mathrm{C} 7-\mathrm{C} 8 & 112.0(2) & \mathrm{C} 13-\mathrm{C} 12-\mathrm{C} 11 & 120.1(3) \\ \mathrm{O} 4-\mathrm{C} 7-\mathrm{O} 3 & 122.5(2) & \mathrm{C} 13-\mathrm{C} 12-\mathrm{H} 12 & 119.9(16) \\ \mathrm{O} 4-\mathrm{C} 7-\mathrm{C} 8 & 125.4(2) & \mathrm{C} 11-\mathrm{C} 12-\mathrm{H} 12 & 119.9(16) \\ \mathrm{N} 1-\mathrm{C} 5-\mathrm{C} 6 & 112.4(2) & \mathrm{C} 12-\mathrm{C} 11-\mathrm{H} 11 & 119.0(16) \\ \mathrm{N} 1-\mathrm{C} 5-\mathrm{H} 5 \mathrm{~A} & 107.7(13) & \mathrm{C} 10-\mathrm{C} 11-\mathrm{C} 12 & 119.6(3) \\ \text { N1-C5-H5B } & 108.7(13) & \mathrm{C} 10-\mathrm{C} 11-\mathrm{H} 11 & 121.3(16) \\ \mathrm{C} 6-\mathrm{C} 5-\mathrm{H} 5 \mathrm{~A} & 106.7(13) & \mathrm{C} 9-\mathrm{C} 10-\mathrm{H} 10 & 116.6(16) \\ \mathrm{C} 6-\mathrm{C} 5-\mathrm{H} 5 \mathrm{~B} & 109.2(13) & \mathrm{C} 11-\mathrm{C} 10-\mathrm{C} 9 & 121.0(3) \\ \mathrm{H} 5 \mathrm{~A}-\mathrm{C} 5-\mathrm{H} 5 \mathrm{~B} & 112.1(18) & \mathrm{C} 11-\mathrm{C} 10-\mathrm{H} 10 & 122.3(15) \\ \mathrm{N} 2-\mathrm{C} 2-\mathrm{H} 2 \mathrm{~A} & 121.9(15) & \mathrm{C} 1-\mathrm{C} 4-\mathrm{H} 4 \mathrm{~A} & 110.6(19) \\ \mathrm{C} 3-\mathrm{C} 2-\mathrm{N} 2 & 106.3(2) & \mathrm{C} 1-\mathrm{C} 4-\mathrm{H} 4 \mathrm{~B} & 111.2(19) \\ \mathrm{C} 3-\mathrm{C} 2-\mathrm{H} 2 \mathrm{~A} & 131.7(15) & \mathrm{C} 1-\mathrm{C} 4-\mathrm{H} 4 \mathrm{C} & 110(2) \\ \mathrm{O} 3-\mathrm{C} 6-\mathrm{C} 5 & 111.9(2) & \mathrm{H} 4 \mathrm{~A}-\mathrm{C} 4-\mathrm{H} 4 \mathrm{~B} & 113(3) \\ \mathrm{O} 3-\mathrm{C} 6-\mathrm{H} 6 \mathrm{~A} & 107.9(15) & \mathrm{H} 4 \mathrm{~A}-\mathrm{C} 4-\mathrm{H} 4 \mathrm{C} & 106(3) \\ \text { O3-C6-H6B } & 102.6(15) & \mathrm{H} 4 \mathrm{~B}-\mathrm{C} 4-\mathrm{H} 4 \mathrm{C} & \end{array}$

2-(2-Methyl-5-nitro-1H-imidazol-1-yl)ethyl benzoate-isophthalic acid (1/1) (bzmdiac)

\section{Crystal data}

$\mathrm{C}_{13} \mathrm{H}_{13} \mathrm{~N}_{3} \mathrm{O}_{4} \cdot \mathrm{C}_{8} \mathrm{H}_{6} \mathrm{O}_{4}$

$M_{r}=441.39$

Monoclinic, $P 2{ }_{1} / c$

$a=8.8300(3) \AA$

$b=33.7182(10) \AA$

$c=7.3199(2) \AA$

$\beta=107.878(1)^{\circ}$

$V=2074.13(11) \AA^{3}$

$Z=4$

\section{Data collection}

Bruker D8 VENTURE Kappa Duo PHOTON II CPAD

diffractometer

Multilayer mirrors monochromator

$\varphi$ and $\omega$ scans

Absorption correction: multi-scan

(SADABS; Bruker, 2016)

$T_{\min }=0.649, T_{\max }=0.746$

\section{Refinement}

Refinement on $F^{2}$

Least-squares matrix: full

$R\left[F^{2}>2 \sigma\left(F^{2}\right)\right]=0.050$

$w R\left(F^{2}\right)=0.145$

$S=1.03$

6344 reflections

365 parameters

0 restraints
$F(000)=920$

$D_{\mathrm{x}}=1.414 \mathrm{Mg} \mathrm{m}^{-3}$

Mo $K \alpha$ radiation, $\lambda=0.71073 \AA$

Cell parameters from 9928 reflections

$\theta=2.4-30.4^{\circ}$

$\mu=0.11 \mathrm{~mm}^{-1}$

$T=304 \mathrm{~K}$

Prism, clear light colourless

$0.95 \times 0.3 \times 0.11 \mathrm{~mm}$

38842 measured reflections

6344 independent reflections

4809 reflections with $I>2 \sigma(I)$

$R_{\text {int }}=0.037$

$\theta_{\max }=30.5^{\circ}, \theta_{\min }=3.0^{\circ}$

$h=-12 \rightarrow 12$

$k=-48 \rightarrow 48$

$l=-10 \rightarrow 10$

Hydrogen site location: difference Fourier map

All $\mathrm{H}$-atom parameters refined

$w=1 /\left[\sigma^{2}\left(F_{\mathrm{o}}^{2}\right)+(0.0601 P)^{2}+0.4881 P\right]$

where $P=\left(F_{\mathrm{o}}^{2}+2 F_{\mathrm{c}}^{2}\right) / 3$

$(\Delta / \sigma)_{\max }<0.001$

$\Delta \rho_{\max }=0.20 \mathrm{e} \AA^{-3}$

$\Delta \rho_{\min }=-0.21$ e $\AA^{-3}$ 


\section{Special details}

Geometry. All esds (except the esd in the dihedral angle between two 1.s. planes) are estimated using the full covariance matrix. The cell esds are taken into account individually in the estimation of esds in distances, angles and torsion angles; correlations between esds in cell parameters are only used when they are defined by crystal symmetry. An approximate (isotropic) treatment of cell esds is used for estimating esds involving l.s. planes.

Refinement. Single-crystal X-ray diffraction data collection ( scans and $\omega$ scans with $\kappa$ and $\theta$ offsets) were performed at room temperature on a Bruker AXS D8 VENTURE equipped with a Kappa goniometer, PHOTON II CPAD detector, a Mo $K \alpha$ INCOATEC I $\mu$ S 3.0 microfocus source $(\lambda=0.71073 \AA)$. The crystal centring, unit-cell determination, refinement of the unit-cell parameters and data collection was controlled through the program APEX3 (Bruker, 2012). The frame integration was performed using SAINT (Bruker, 2016) and the intensities were scaled and absorption corrected using SADABS (Bruker, 2001). Using OLEX2 (Dolomanov et al., 2009), the structure was solved by intrinsic phasing using SHELXT (Sheldrick, 2015) and refined by full-matrix least-squares calculation based on $F^{2}$ for all reflection using SHELXL (Sheldrick, 2007). All non-H atoms were refined anisotropically.

Fractional atomic coordinates and isotropic or equivalent isotropic displacement parameters $\left(\AA^{2}\right)$

\begin{tabular}{|c|c|c|c|c|}
\hline & $x$ & $y$ & $z$ & $U_{\text {iso }} * / U_{\text {eq }}$ \\
\hline $\mathrm{O} 3$ & $0.36882(11)$ & $0.28188(3)$ & $-0.12385(16)$ & $0.0549(3)$ \\
\hline $\mathrm{O} 4^{\prime}$ & $1.31886(13)$ & $0.48005(4)$ & $0.42956(16)$ & $0.0617(3)$ \\
\hline $\mathrm{O} 1^{\prime}$ & $0.78049(13)$ & $0.42754(4)$ & $0.32394(16)$ & $0.0610(3)$ \\
\hline $\mathrm{O}^{\prime}$ & $1.47167(13)$ & $0.49211(4)$ & $0.72771(17)$ & $0.0697(4)$ \\
\hline N1 & $0.28442(13)$ & $0.36122(3)$ & $-0.03743(16)$ & $0.0452(3)$ \\
\hline $\mathrm{O} 1$ & $0.12528(17)$ & $0.37021(4)$ & $0.3517(2)$ & $0.0760(4)$ \\
\hline N2 & $0.48973(15)$ & $0.39681(4)$ & $0.13450(19)$ & $0.0537(3)$ \\
\hline N3 & $0.12377(16)$ & $0.36110(4)$ & $0.1893(2)$ & $0.0550(3)$ \\
\hline $\mathrm{O} 2$ & $0.01225(15)$ & $0.34423(5)$ & $0.0737(2)$ & $0.0833(4)$ \\
\hline $\mathrm{O} 4$ & $0.30372(15)$ & $0.21816(4)$ & $-0.1125(3)$ & $0.0846(4)$ \\
\hline $\mathrm{O} 2^{\prime}$ & $0.68960(17)$ & $0.41614(6)$ & $0.5685(2)$ & $0.0982(6)$ \\
\hline $\mathrm{C}^{\prime}$ & $1.07098(15)$ & $0.45287(4)$ & $0.5617(2)$ & $0.0431(3)$ \\
\hline $\mathrm{C}^{\prime}$ & $1.21363(15)$ & $0.46743(4)$ & 0.68527 (19) & $0.0438(3)$ \\
\hline $\mathrm{C} 3$ & $0.26003(16)$ & $0.37089(4)$ & $0.1350(2)$ & $0.0440(3)$ \\
\hline $\mathrm{C} 8^{\prime}$ & $1.33969(15)$ & $0.48031(4)$ & $0.6041(2)$ & $0.0476(3)$ \\
\hline $\mathrm{C} 1^{\prime}$ & $0.95064(16)$ & $0.44156(4)$ & $0.6348(2)$ & $0.0455(3)$ \\
\hline $\mathrm{C} 8$ & $0.56805(17)$ & $0.23453(4)$ & $-0.1057(2)$ & $0.0491(3)$ \\
\hline $\mathrm{C} 1$ & $0.42668(17)$ & $0.37765(4)$ & $-0.0295(2)$ & $0.0507(3)$ \\
\hline $\mathrm{C} 2$ & $0.38648(18)$ & $0.39278(4)$ & $0.2375(2)$ & 0.0509 (3) \\
\hline $\mathrm{C} 7$ & $0.40177(17)$ & $0.24289(5)$ & $-0.1124(2)$ & $0.0526(3)$ \\
\hline $\mathrm{C} 4^{\prime}$ & $1.23414(18)$ & $0.47070(5)$ & $0.8801(2)$ & $0.0522(3)$ \\
\hline$C 7^{\prime}$ & $0.79402(18)$ & $0.42688(5)$ & $0.5072(2)$ & $0.0539(3)$ \\
\hline $\mathrm{C} 5$ & 0.18737 (19) & $0.33572(5)$ & $-0.1921(2)$ & $0.0544(3)$ \\
\hline $\mathrm{C} 2^{\prime}$ & $0.97138(19)$ & $0.44485(5)$ & $0.8302(2)$ & $0.0552(3)$ \\
\hline C6 & $0.20736(17)$ & $0.29250(5)$ & -0.1403 & $0.0539(4)$ \\
\hline C9 & $0.67651(18)$ & $0.26461(5)$ & $-0.0982(3)$ & $0.0578(4)$ \\
\hline $\mathrm{C} 3^{\prime}$ & $1.1133(2)$ & $0.45954(5)$ & $0.9526(2)$ & $0.0585(4)$ \\
\hline $\mathrm{C} 10$ & $0.8293(2)$ & $0.25530(6)$ & $-0.0977(3)$ & $0.0664(4)$ \\
\hline $\mathrm{C} 11$ & $0.8741(2)$ & $0.21669(6)$ & -0.1068 & $0.0664(4)$ \\
\hline $\mathrm{C} 13$ & $0.6152(2)$ & $0.19536(5)$ & $-0.1115(3)$ & 0.0669 (4) \\
\hline C12 & $0.7681(2)$ & $0.18670(6)$ & -0.1130 & $0.0739(5)$ \\
\hline $\mathrm{C} 4$ & $0.4995(3)$ & $0.37538(8)$ & $-0.1860(4)$ & $0.0781(6)$ \\
\hline
\end{tabular}




$\begin{array}{lllll}\text { H6' } & 1.0525(19) & 0.4509(5) & 0.425(2) & 0.049(4)^{*} \\ \text { H5A } & 0.219(2) & 0.3419(5) & -0.307(3) & 0.059(5)^{*} \\ \text { H5B } & 0.077(2) & 0.3443(5) & -0.217(3) & 0.060(5)^{*} \\ \text { H6A } & 0.138(2) & 0.2775(5) & -0.242(3) & 0.062(5)^{*} \\ \text { H2 } & 0.405(2) & 0.4048(6) & 0.365(3) & 0.066(5)^{*} \\ \text { H4' } & 1.335(2) & 0.4810(5) & 0.964(3) & 0.059(5)^{*} \\ \text { H6B } & 0.187(2) & 0.2868(5) & -0.018(3) & 0.060(5)^{*} \\ \text { H3'A } & 1.128(2) & 0.4626(6) & 1.089(3) & 0.072(5)^{*} \\ \text { H9 } & 0.645(2) & 0.2931(6) & -0.091(3) & 0.076(6)^{*} \\ \text { H13 } & 0.538(3) & 0.1757(7) & -0.116(3) & 0.083(6)^{*} \\ \text { H2' } & 0.881(2) & 0.4366(6) & 0.884(3) & 0.074(6)^{*} \\ \text { H1' } & 0.683(3) & 0.4171(7) & 0.249(3) & 0.099(7)^{*} \\ \text { H11 } & 0.983(3) & 0.2095(6) & -0.109(3) & 0.084(6)^{*} \\ \text { H10 } & 0.906(3) & 0.2768(6) & -0.092(3) & 0.080(6)^{*} \\ \text { H12 } & 0.796(3) & 0.1580(7) & -0.126(3) & 0.091(7)^{*} \\ \text { H4A } & 0.493(4) & 0.3484(12) & -0.248(5) & 0.150(12)^{*} \\ \text { H4B } & 0.598(5) & 0.3788(12) & -0.136(5) & 0.154(14)^{*} \\ \text { H3' } & 1.543(4) & 0.5032(9) & 0.655(4) & 0.125(9)^{*} \\ \text { H4C } & 0.469(5) & 0.3945(12) & -0.266(6) & 0.163(15)^{*}\end{array}$

Atomic displacement parameters $\left(\AA^{2}\right)$

\begin{tabular}{lllllll}
\hline & $U^{11}$ & $U^{22}$ & $U^{33}$ & $U^{12}$ & $U^{13}$ & $U^{23}$ \\
\hline O3 & $0.0382(5)$ & $0.0566(6)$ & $0.0703(7)$ & $-0.0101(4)$ & $0.0171(5)$ & $-0.0127(5)$ \\
O4 $^{\prime}$ & $0.0450(5)$ & $0.0863(8)$ & $0.0503(6)$ & $-0.0193(5)$ & $0.0093(5)$ & $-0.0118(5)$ \\
O1' $^{\prime}$ & $0.0457(5)$ & $0.0788(8)$ & $0.0533(6)$ & $-0.0230(5)$ & $0.0073(5)$ & $-0.0005(5)$ \\
O3 $^{\prime}$ & $0.0412(5)$ & $0.1084(10)$ & $0.0535(6)$ & $-0.0234(6)$ & $0.0055(5)$ & $-0.0159(6)$ \\
N1 & $0.0418(5)$ & $0.0493(6)$ & $0.0435(6)$ & $-0.0070(5)$ & $0.0115(4)$ & $0.0015(5)$ \\
O1 & $0.0815(9)$ & $0.0851(9)$ & $0.0766(8)$ & $-0.0039(7)$ & $0.0465(7)$ & $-0.0137(7)$ \\
N2 & $0.0459(6)$ & $0.0519(7)$ & $0.0593(7)$ & $-0.0118(5)$ & $0.0102(5)$ & $0.0050(5)$ \\
N3 & $0.0505(7)$ & $0.0522(7)$ & $0.0681(8)$ & $-0.0035(5)$ & $0.0269(6)$ & $-0.0042(6)$ \\
O2 & $0.0555(7)$ & $0.1038(10)$ & $0.0973(10)$ & $-0.0306(7)$ & $0.0334(7)$ & $-0.0289(8)$ \\
O4 & $0.0567(7)$ & $0.0643(8)$ & $0.1410(13)$ & $-0.0193(6)$ & $0.0427(8)$ & $-0.0104(8)$ \\
O2' & $0.0695(8)$ & $0.1590(15)$ & $0.0670(8)$ & $-0.0616(9)$ & $0.0225(7)$ & $-0.0070(9)$ \\
C6' & $0.0385(6)$ & $0.0428(6)$ & $0.0438(7)$ & $-0.0046(5)$ & $0.0067(5)$ & $-0.0041(5)$ \\
C5' & $0.0375(6)$ & $0.0416(6)$ & $0.0479(7)$ & $-0.0009(5)$ & $0.0067(5)$ & $-0.0034(5)$ \\
C3 & $0.0405(6)$ & $0.0434(6)$ & $0.0481(7)$ & $-0.0028(5)$ & $0.0134(5)$ & $0.0009(5)$ \\
C8 & $0.0353(6)$ & $0.0517(7)$ & $0.0497(7)$ & $-0.0039(5)$ & $0.0041(5)$ & $-0.0087(6)$ \\
C1' & $0.0413(6)$ & $0.0425(6)$ & $0.0497(7)$ & $-0.0051(5)$ & $0.0092(5)$ & $-0.0002(5)$ \\
C8 & $0.0444(7)$ & $0.0548(8)$ & $0.0476(7)$ & $-0.0079(6)$ & $0.0135(6)$ & $-0.0088(6)$ \\
C1 & $0.0459(7)$ & $0.0529(8)$ & $0.0538(8)$ & $-0.0077(6)$ & $0.0162(6)$ & $0.0088(6)$ \\
C2 & $0.0500(7)$ & $0.0476(7)$ & $0.0517(8)$ & $-0.0063(6)$ & $0.0104(6)$ & $-0.0012(6)$ \\
C7 & $0.0454(7)$ & $0.0569(8)$ & $0.0566(8)$ & $-0.0129(6)$ & $0.0170(6)$ & $-0.0117(6)$ \\
C4' & $0.0453(7)$ & $0.0558(8)$ & $0.0486(7)$ & $-0.0023(6)$ & $0.0040(6)$ & $-0.0054(6)$ \\
C7' & $0.0463(7)$ & $0.0564(8)$ & $0.0570(8)$ & $-0.0159(6)$ & $0.0128(6)$ & $0.0003(6)$ \\
C5 & $0.0444(7)$ & $0.0689(9)$ & $0.0445(7)$ & $-0.0055(7)$ & $0.0054(6)$ & $-0.0050(6)$ \\
C2' & $0.0510(8)$ & $0.0609(9)$ & $0.0536(8)$ & $-0.0032(7)$ & $0.0158(7)$ & $0.0033(7)$ \\
C6 & $0.0353(6)$ & $0.0627(9)$ & $0.0614(9)$ & $-0.0116(6)$ & $0.0115(6)$ & $-0.0147(7)$ \\
& & & & &
\end{tabular}




\begin{tabular}{lllllll} 
C9 & $0.0441(7)$ & $0.0587(9)$ & $0.0722(10)$ & $-0.0089(6)$ & $0.0201(7)$ & $-0.0106(7)$ \\
C3' & $0.0577(9)$ & $0.0701(10)$ & $0.0446(7)$ & $-0.0016(7)$ & $0.0111(6)$ & $-0.0018(7)$ \\
C10 & $0.0454(8)$ & $0.0737(11)$ & $0.0820(12)$ & $-0.0092(8)$ & $0.0224(8)$ & $-0.0097(9)$ \\
C11 & $0.0487(8)$ & $0.0821(12)$ & $0.0681(10)$ & $0.0060(8)$ & $0.0177(8)$ & $-0.0065(9)$ \\
C13 & $0.0632(10)$ & $0.0555(9)$ & $0.0840(12)$ & $-0.0094(8)$ & $0.0254(9)$ & $-0.0075(8)$ \\
C12 & $0.0688(11)$ & $0.0645(11)$ & $0.0887(13)$ & $0.0105(9)$ & $0.0250(10)$ & $-0.0045(9)$ \\
C4 & $0.0825(15)$ & $0.0893(15)$ & $0.0776(13)$ & $-0.0158(12)$ & $0.0468(12)$ & $0.0077(12)$ \\
\hline
\end{tabular}

Geometric parameters $\left(\AA,{ }^{\circ}\right)$

\begin{tabular}{|c|c|c|c|}
\hline $\mathrm{O} 3-\mathrm{C} 7$ & $1.3435(19)$ & $\mathrm{C} 8-\mathrm{C} 9$ & $1.384(2)$ \\
\hline $\mathrm{O} 3-\mathrm{C} 6$ & $1.4388(17)$ & $\mathrm{C} 8-\mathrm{C} 13$ & $1.389(2)$ \\
\hline $\mathrm{O} 4^{\prime}-\mathrm{C}^{\prime}$ & $1.2338(18)$ & $\mathrm{C} 1-\mathrm{C} 4$ & $1.478(3)$ \\
\hline $\mathrm{O} 1^{\prime}-\mathrm{C}^{\prime}$ & $1.3101(19)$ & $\mathrm{C} 2-\mathrm{H} 2$ & 0.983 (19) \\
\hline $\mathrm{O} 1^{\prime}-\mathrm{H} 1^{\prime}$ & $0.93(3)$ & $\mathrm{C} 4^{\prime}-\mathrm{C} 3^{\prime}$ & $1.382(2)$ \\
\hline $\mathrm{O} 3^{\prime}-\mathrm{C}^{\prime}$ & $1.2991(16)$ & $\mathrm{C} 4^{\prime}-\mathrm{H} 4^{\prime}$ & 0.973 (19) \\
\hline $\mathrm{O} 3^{\prime}-\mathrm{H} 3^{\prime}$ & $1.02(3)$ & $\mathrm{C} 5-\mathrm{C} 6$ & $1.502(2)$ \\
\hline $\mathrm{N} 1-\mathrm{C} 3$ & $1.3829(18)$ & $\mathrm{C} 5-\mathrm{H} 5 \mathrm{~A}$ & 0.989 (18) \\
\hline $\mathrm{N} 1-\mathrm{C} 1$ & $1.3578(17)$ & $\mathrm{C} 5-\mathrm{H} 5 \mathrm{~B}$ & $0.976(18)$ \\
\hline $\mathrm{N} 1-\mathrm{C} 5$ & $1.4694(18)$ & $\mathrm{C} 2^{\prime}-\mathrm{C} 3^{\prime}$ & $1.389(2)$ \\
\hline $\mathrm{O} 1-\mathrm{N} 3$ & $1.2243(18)$ & $\mathrm{C} 2^{\prime}-\mathrm{H} 2^{\prime}$ & $1.03(2)$ \\
\hline $\mathrm{N} 2-\mathrm{C} 1$ & $1.326(2)$ & C6-H6A & 0.952 (19) \\
\hline $\mathrm{N} 2-\mathrm{C} 2$ & $1.357(2)$ & C6-H6B & $0.988(18)$ \\
\hline $\mathrm{N} 3-\mathrm{O} 2$ & $1.2236(18)$ & $\mathrm{C} 9-\mathrm{C} 10$ & $1.384(2)$ \\
\hline $\mathrm{N} 3-\mathrm{C} 3$ & $1.4171(18)$ & C9- $\mathrm{H} 9$ & $1.01(2)$ \\
\hline $\mathrm{O} 4-\mathrm{C} 7$ & $1.2019(18)$ & $\mathrm{C} 3^{\prime}-\mathrm{H} 3^{\prime} \mathrm{A}$ & $0.97(2)$ \\
\hline $\mathrm{O} 2^{\prime}-\mathrm{C}^{\prime}$ & $1.1986(19)$ & $\mathrm{C} 10-\mathrm{C} 11$ & $1.368(3)$ \\
\hline $\mathrm{C} 6^{\prime}-\mathrm{C} 5^{\prime}$ & $1.3953(18)$ & $\mathrm{C} 10-\mathrm{H} 10$ & $0.98(2)$ \\
\hline $\mathrm{C} 6^{\prime}-\mathrm{C} 1^{\prime}$ & $1.3821(19)$ & $\mathrm{C} 11-\mathrm{C} 12$ & $1.369(3)$ \\
\hline $\mathrm{C} 6^{\prime}-\mathrm{H} 6^{\prime}$ & $0.968(16)$ & C11-H11 & $1.00(2)$ \\
\hline $\mathrm{C} 5^{\prime}-\mathrm{C} 8^{\prime}$ & $1.479(2)$ & $\mathrm{C} 13-\mathrm{C} 12$ & $1.385(3)$ \\
\hline $\mathrm{C} 5^{\prime}-\mathrm{C} 4^{\prime}$ & $1.386(2)$ & C13-H13 & $0.94(2)$ \\
\hline $\mathrm{C} 3-\mathrm{C} 2$ & $1.3564(19)$ & $\mathrm{C} 12-\mathrm{H} 12$ & $1.01(2)$ \\
\hline $\mathrm{C} 1^{\prime}-\mathrm{C} 7^{\prime}$ & $1.4955(19)$ & $\mathrm{C} 4-\mathrm{H} 4 \mathrm{~A}$ & $1.01(4)$ \\
\hline $\mathrm{C} 1^{\prime}-\mathrm{C} 2^{\prime}$ & $1.389(2)$ & $\mathrm{C} 4-\mathrm{H} 4 \mathrm{~B}$ & $0.84(4)$ \\
\hline $\mathrm{C} 8-\mathrm{C} 7$ & $1.481(2)$ & $\mathrm{C} 4-\mathrm{H} 4 \mathrm{C}$ & $0.86(4)$ \\
\hline $\mathrm{C} 7-\mathrm{O} 3-\mathrm{C} 6$ & $116.03(11)$ & $\mathrm{O} 2^{\prime}-\mathrm{C}^{\prime}-\mathrm{O} 1^{\prime}$ & $123.34(14)$ \\
\hline $\mathrm{C} 7^{\prime}-\mathrm{O} 1^{\prime}-\mathrm{H} 1^{\prime}$ & $111.7(15)$ & $\mathrm{O} 2^{\prime}-\mathrm{C} 7^{\prime}-\mathrm{C} 1^{\prime}$ & $122.45(15)$ \\
\hline $\mathrm{C} 8^{\prime}-\mathrm{O}^{\prime}-\mathrm{H} 3^{\prime}$ & $108.4(16)$ & $\mathrm{N} 1-\mathrm{C} 5-\mathrm{C} 6$ & $112.20(13)$ \\
\hline $\mathrm{C} 3-\mathrm{N} 1-\mathrm{C} 5$ & $128.58(12)$ & $\mathrm{N} 1-\mathrm{C} 5-\mathrm{H} 5 \mathrm{~A}$ & $106.5(10)$ \\
\hline $\mathrm{C} 1-\mathrm{N} 1-\mathrm{C} 3$ & $105.42(11)$ & $\mathrm{N} 1-\mathrm{C} 5-\mathrm{H} 5 \mathrm{~B}$ & $106.3(10)$ \\
\hline $\mathrm{C} 1-\mathrm{N} 1-\mathrm{C} 5$ & $125.80(13)$ & $\mathrm{C} 6-\mathrm{C} 5-\mathrm{H} 5 \mathrm{~A}$ & $112.2(10)$ \\
\hline $\mathrm{C} 1-\mathrm{N} 2-\mathrm{C} 2$ & $106.69(12)$ & $\mathrm{C} 6-\mathrm{C} 5-\mathrm{H} 5 \mathrm{~B}$ & $111.5(10)$ \\
\hline $\mathrm{O} 1-\mathrm{N} 3-\mathrm{C} 3$ & $117.19(13)$ & $\mathrm{H} 5 \mathrm{~A}-\mathrm{C} 5-\mathrm{H} 5 \mathrm{~B}$ & $107.7(15)$ \\
\hline $\mathrm{O} 2-\mathrm{N} 3-\mathrm{O} 1$ & $123.67(14)$ & $\mathrm{C} 1^{\prime}-\mathrm{C} 2^{\prime}-\mathrm{H} 2^{\prime}$ & $120.0(11)$ \\
\hline $\mathrm{O} 2-\mathrm{N} 3-\mathrm{C} 3$ & $119.14(13)$ & $\mathrm{C} 3^{\prime}-\mathrm{C} 2^{\prime}-\mathrm{C} 1^{\prime}$ & $120.03(15)$ \\
\hline $\mathrm{C} 5^{\prime}-\mathrm{C} 6^{\prime}-\mathrm{H} 6^{\prime}$ & $121.9(10)$ & $\mathrm{C} 3^{\prime}-\mathrm{C} 2^{\prime}-\mathrm{H} 2^{\prime}$ & $119.9(11)$ \\
\hline
\end{tabular}




\begin{tabular}{|c|c|c|c|}
\hline $\mathrm{C} 1^{\prime}-\mathrm{C} 6^{\prime}-\mathrm{C} 5^{\prime}$ & $119.62(13)$ & $\mathrm{O} 3-\mathrm{C} 6-\mathrm{C} 5$ & $107.41(12)$ \\
\hline $\mathrm{C} 1^{\prime}-\mathrm{C} 6^{\prime}-\mathrm{H} 6^{\prime}$ & $118.5(10)$ & $\mathrm{O} 3-\mathrm{C} 6-\mathrm{H} 6 \mathrm{~A}$ & $108.6(11)$ \\
\hline $\mathrm{C} 6^{\prime}-\mathrm{C} 5^{\prime}-\mathrm{C} 8^{\prime}$ & $118.94(12)$ & $\mathrm{O} 3-\mathrm{C} 6-\mathrm{H} 6 \mathrm{~B}$ & $109.3(11)$ \\
\hline $\mathrm{C} 4^{\prime}-\mathrm{C} 5^{\prime}-\mathrm{C} 6^{\prime}$ & $120.14(13)$ & $\mathrm{C} 5-\mathrm{C} 6-\mathrm{H} 6 \mathrm{~A}$ & $108.8(11)$ \\
\hline $\mathrm{C} 4^{\prime}-\mathrm{C} 5^{\prime}-\mathrm{C} 8^{\prime}$ & $120.90(12)$ & $\mathrm{C} 5-\mathrm{C} 6-\mathrm{H} 6 \mathrm{~B}$ & $112.1(10)$ \\
\hline $\mathrm{N} 1-\mathrm{C} 3-\mathrm{N} 3$ & $125.76(12)$ & $\mathrm{H} 6 \mathrm{~A}-\mathrm{C} 6-\mathrm{H} 6 \mathrm{~B}$ & $110.6(15)$ \\
\hline $\mathrm{C} 2-\mathrm{C} 3-\mathrm{N} 1$ & $107.51(12)$ & $\mathrm{C} 8-\mathrm{C} 9-\mathrm{H} 9$ & $120.0(12)$ \\
\hline $\mathrm{C} 2-\mathrm{C} 3-\mathrm{N} 3$ & $126.67(13)$ & $\mathrm{C} 10-\mathrm{C} 9-\mathrm{C} 8$ & $119.72(16)$ \\
\hline $\mathrm{O}^{\prime}-\mathrm{C} 8^{\prime}-\mathrm{O} 3^{\prime}$ & $122.69(14)$ & $\mathrm{C} 10-\mathrm{C} 9-\mathrm{H} 9$ & $120.3(12)$ \\
\hline $\mathrm{O} 4^{\prime}-\mathrm{C} 8^{\prime}-\mathrm{C}^{\prime}$ & $121.51(12)$ & $\mathrm{C} 4^{\prime}-\mathrm{C} 3^{\prime}-\mathrm{C} 2^{\prime}$ & $119.96(15)$ \\
\hline $\mathrm{O} 3^{\prime}-\mathrm{C}^{\prime}-\mathrm{C}^{\prime}$ & $115.80(13)$ & $\mathrm{C}^{\prime}-\mathrm{C}^{\prime}-\mathrm{H} 3^{\prime} \mathrm{A}$ & $119.1(12)$ \\
\hline $\mathrm{C} 6^{\prime}-\mathrm{C} 1^{\prime}-\mathrm{C}^{\prime}$ & $121.54(13)$ & $\mathrm{C} 2^{\prime}-\mathrm{C}^{\prime}-\mathrm{H} 3^{\prime} \mathrm{A}$ & $120.9(12)$ \\
\hline $\mathrm{C} 6^{\prime}-\mathrm{C} 1^{\prime}-\mathrm{C} 2^{\prime}$ & $120.17(13)$ & $\mathrm{C} 9-\mathrm{C} 10-\mathrm{H} 10$ & $119.3(13)$ \\
\hline $\mathrm{C} 2^{\prime}-\mathrm{C} 1^{\prime}-\mathrm{C} 7^{\prime}$ & $118.26(13)$ & $\mathrm{C} 11-\mathrm{C} 10-\mathrm{C} 9$ & $120.78(17)$ \\
\hline $\mathrm{C} 9-\mathrm{C} 8-\mathrm{C} 7$ & $121.91(14)$ & $\mathrm{C} 11-\mathrm{C} 10-\mathrm{H} 10$ & $119.9(13)$ \\
\hline $\mathrm{C} 9-\mathrm{C} 8-\mathrm{C} 13$ & $119.19(15)$ & $\mathrm{C} 10-\mathrm{C} 11-\mathrm{C} 12$ & $119.97(17)$ \\
\hline $\mathrm{C} 13-\mathrm{C} 8-\mathrm{C} 7$ & $118.88(14)$ & $\mathrm{C} 10-\mathrm{C} 11-\mathrm{H} 11$ & $121.8(12)$ \\
\hline $\mathrm{N} 1-\mathrm{C} 1-\mathrm{C} 4$ & $124.24(16)$ & $\mathrm{C} 12-\mathrm{C} 11-\mathrm{H} 11$ & $118.2(12)$ \\
\hline $\mathrm{N} 2-\mathrm{C} 1-\mathrm{N} 1$ & $111.31(13)$ & $\mathrm{C} 8-\mathrm{C} 13-\mathrm{H} 13$ & $116.8(13)$ \\
\hline $\mathrm{N} 2-\mathrm{C} 1-\mathrm{C} 4$ & $124.42(16)$ & $\mathrm{C} 12-\mathrm{C} 13-\mathrm{C} 8$ & $120.17(17)$ \\
\hline $\mathrm{N} 2-\mathrm{C} 2-\mathrm{C} 3$ & $109.07(13)$ & $\mathrm{C} 12-\mathrm{C} 13-\mathrm{H} 13$ & $123.1(13)$ \\
\hline $\mathrm{N} 2-\mathrm{C} 2-\mathrm{H} 2$ & $122.9(11)$ & $\mathrm{C} 11-\mathrm{C} 12-\mathrm{C} 13$ & $120.15(18)$ \\
\hline $\mathrm{C} 3-\mathrm{C} 2-\mathrm{H} 2$ & $128.1(11)$ & $\mathrm{C} 11-\mathrm{C} 12-\mathrm{H} 12$ & $121.6(13)$ \\
\hline $\mathrm{O} 3-\mathrm{C} 7-\mathrm{C} 8$ & $112.45(12)$ & $\mathrm{C} 13-\mathrm{C} 12-\mathrm{H} 12$ & $118.2(13)$ \\
\hline $\mathrm{O} 4-\mathrm{C} 7-\mathrm{O} 3$ & $122.45(14)$ & $\mathrm{C} 1-\mathrm{C} 4-\mathrm{H} 4 \mathrm{~A}$ & 114.7 (19) \\
\hline $\mathrm{O} 4-\mathrm{C} 7-\mathrm{C} 8$ & $125.08(15)$ & $\mathrm{C} 1-\mathrm{C} 4-\mathrm{H} 4 \mathrm{~B}$ & $107(2)$ \\
\hline $\mathrm{C} 5^{\prime}-\mathrm{C} 4^{\prime}-\mathrm{H} 4^{\prime}$ & $118.9(10)$ & $\mathrm{C} 1-\mathrm{C} 4-\mathrm{H} 4 \mathrm{C}$ & $112(3)$ \\
\hline $\mathrm{C} 3^{\prime}-\mathrm{C} 4^{\prime}-\mathrm{C} 5^{\prime}$ & $120.08(14)$ & $\mathrm{H} 4 \mathrm{~A}-\mathrm{C} 4-\mathrm{H} 4 \mathrm{~B}$ & $103(3)$ \\
\hline $\mathrm{C} 3^{\prime}-\mathrm{C} 4^{\prime}-\mathrm{H} 4^{\prime}$ & $121.1(10)$ & $\mathrm{H} 4 \mathrm{~A}-\mathrm{C} 4-\mathrm{H} 4 \mathrm{C}$ & $114(3)$ \\
\hline $\mathrm{O} 1^{\prime}-\mathrm{C} 7^{\prime}-\mathrm{C} 1^{\prime}$ & $114.20(12)$ & $\mathrm{H} 4 \mathrm{~B}-\mathrm{C} 4-\mathrm{H} 4 \mathrm{C}$ & $105(3)$ \\
\hline
\end{tabular}

2-(2-Methyl-5-nitro-1H-imidazol-1-yl)ethyl benzoate-resorcinol (2/1) (bzmdres)

Crystal data

$2 \mathrm{C}_{13} \mathrm{H}_{13} \mathrm{~N}_{3} \mathrm{O}_{4} \cdot \mathrm{C}_{6} \mathrm{H}_{6} \mathrm{O}_{2}$

$M_{r}=660.63$

$D_{\mathrm{x}}=1.376 \mathrm{Mg} \mathrm{m}^{-3}$

Orthorhombic, $\mathrm{Pbca}$

$a=26.3241$ (4) $\AA$

$b=7.1612(1) \AA$

$c=33.8433(5) \AA$

$V=6379.87(16) \AA^{3}$

$Z=8$

$\mathrm{Cu} K \alpha$ radiation, $\lambda=1.54178 \AA$

Cell parameters from 9896 reflections

$\theta=3.1-68.2^{\circ}$

$\mu=0.88 \mathrm{~mm}^{-1}$

$T=300 \mathrm{~K}$

Plate, clear light colourless

$F(000)=2768$

$0.25 \times 0.12 \times 0.11 \mathrm{~mm}$

Data collection

Bruker APEXII CCD

diffractometer

$\varphi$ and $\omega$ scans

Absorption correction: multi-scan

(SADABS; Bruker, 2016)

$T_{\min }=0.623, T_{\max }=0.753$

121051 measured reflections

5865 independent reflections

4816 reflections with $I>2 \sigma(I)$

$R_{\text {int }}=0.066$ 
$\theta_{\text {max }}=68.3^{\circ}, \theta_{\text {min }}=2.6^{\circ}$

$h=-31 \rightarrow 31$

\section{Refinement}

Refinement on $F^{2}$

Least-squares matrix: full

$R\left[F^{2}>2 \sigma\left(F^{2}\right)\right]=0.048$

$w R\left(F^{2}\right)=0.147$

$S=1.03$

5865 reflections

540 parameters

0 restraints

Primary atom site location: dual

Hydrogen site location: mixed

$$
\begin{aligned}
& k=-8 \rightarrow 8 \\
& l=-40 \rightarrow 40
\end{aligned}
$$

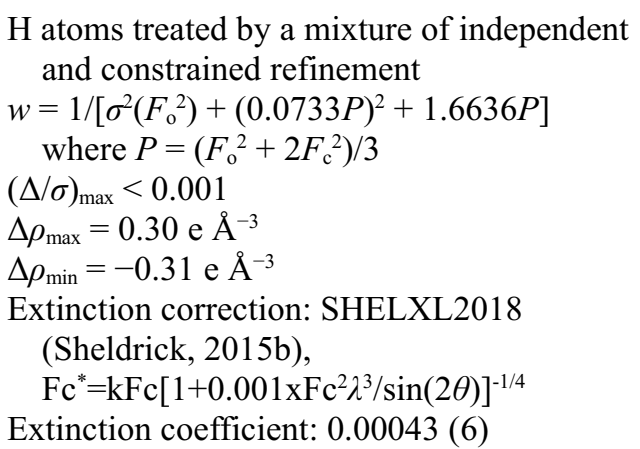

\section{Special details}

Geometry. All esds (except the esd in the dihedral angle between two 1.s. planes) are estimated using the full covariance matrix. The cell esds are taken into account individually in the estimation of esds in distances, angles and torsion angles; correlations between esds in cell parameters are only used when they are defined by crystal symmetry. An approximate (isotropic) treatment of cell esds is used for estimating esds involving 1.s. planes.

Refinement. Single-crystal X-ray diffraction data collection ( $\quad$ scans and $\omega$ scans with $\kappa$ and $\theta$ offsets) were performed at room temperature on a Bruker AXS D8 VENTURE equipped with a Kappa goniometer, PHOTON II CPAD detector, a Mo $K \alpha$ INCOATEC I $\mu$ S 3.0 microfocus source $(\lambda=0.71073 \AA)$. The crystal centring, unit-cell determination, refinement of the unit-cell parameters and data collection was controlled through the program APEX3 (Bruker, 2012). The frame integration was performed using SAINT (Bruker, 2016) and the intensities were scaled and absorption corrected using SADABS (Bruker, 2001). Using OLEX2 (Dolomanov et al., 2009), the structure was solved by intrinsic phasing using SHELXT (Sheldrick, 2015) and refined by full-matrix least-squares calculation based on $F^{2}$ for all reflection using SHELXL (Sheldrick, 2007). All non-H atoms were refined anisotropically.

Fractional atomic coordinates and isotropic or equivalent isotropic displacement parameters $\left(\AA^{2}\right)$

\begin{tabular}{lllll}
\hline & $x$ & $y$ & $z$ & $U_{\text {iso }} * / U_{\text {eq }}$ \\
\hline O3B & $0.29177(5)$ & $0.7763(2)$ & $0.21878(3)$ & $0.0676(3)$ \\
O3A & $0.70575(5)$ & $-0.1189(2)$ & $0.52879(4)$ & $0.0691(3)$ \\
N1A & $0.60485(5)$ & $-0.0111(2)$ & $0.55009(4)$ & $0.0594(4)$ \\
N1B & $0.39439(5)$ & $0.6490(2)$ & $0.19753(4)$ & $0.0606(4)$ \\
O4B & $0.21161(6)$ & $0.7410(3)$ & $0.19886(5)$ & $0.0946(5)$ \\
N2A & $0.55969(6)$ & $0.1485(2)$ & $0.50650(5)$ & $0.0698(4)$ \\
O2' & $0.52718(8)$ & $0.3814(3)$ & $0.44659(5)$ & $0.0978(5)$ \\
O4A & $0.78574(6)$ & $-0.1384(3)$ & $0.55011(5)$ & $0.1011(6)$ \\
N2B & $0.44240(6)$ & $0.5183(3)$ & $0.24363(5)$ & $0.0721(4)$ \\
O1' & $0.48855(7)$ & $0.5514(3)$ & $0.31647(5)$ & $0.1049(6)$ \\
O2A & $0.63071(7)$ & $0.1226(3)$ & $0.62598(5)$ & $0.1110(6)$ \\
N3A & $0.61124(7)$ & $0.2312(3)$ & $0.60258(6)$ & $0.0859(5)$ \\
C8B & $0.22773(7)$ & $0.8238(2)$ & $0.26590(5)$ & $0.0603(4)$ \\
O2B & $0.36980(8)$ & $0.4818(4)$ & $0.12448(6)$ & $0.1210(7)$ \\
N3B & $0.39162(7)$ & $0.3882(3)$ & $0.14929(7)$ & $0.0919(6)$ \\
C8A & $0.77042(7)$ & $-0.0609(2)$ & $0.48271(5)$ & $0.0616(4)$ \\
C3A & $0.59504(6)$ & $0.1642(3)$ & $0.56527(5)$ & $0.0629(4)$ \\
O1A & $0.60434(8)$ & $0.3983(3)$ & $0.60894(7)$ & $0.1274(8)$ \\
C7B & $0.24133(7)$ & $0.7746(3)$ & $0.22482(5)$ & $0.0637(4)$
\end{tabular}




\begin{tabular}{|c|c|c|c|c|}
\hline C1B & $0.41703(7)$ & 0.6707 (3) & $0.23323(5)$ & 0.0633 (4) \\
\hline $\mathrm{C} 1 \mathrm{~A}$ & $0.58274(7)$ & $-0.0115(3)$ & $0.51389(5)$ & $0.0647(4)$ \\
\hline C7A & $0.75635(7)$ & $-0.1104(3)$ & $0.52361(5)$ & $0.0660(5)$ \\
\hline C9A & $0.73439(8)$ & $-0.0105(3)$ & $0.45482(5)$ & 0.0644 (4) \\
\hline $\mathrm{C} 3 \mathrm{~B}$ & $0.40651(6)$ & $0.4703(3)$ & $0.18565(6)$ & $0.0661(5)$ \\
\hline $\mathrm{C} 2 \mathrm{~A}$ & $0.56744(7)$ & $0.2580(3)$ & $0.53836(6)$ & $0.0678(5)$ \\
\hline $\mathrm{C} 6^{\prime}$ & $0.51323(7)$ & $0.3226(3)$ & $0.40973(6)$ & 0.0687 (5) \\
\hline $\mathrm{C} 2^{\prime}$ & $0.49229(7)$ & $0.4103(3)$ & $0.34347(6)$ & $0.0721(5)$ \\
\hline C9B & $0.26411(8)$ & $0.8558(3)$ & $0.29468(6)$ & $0.0689(5)$ \\
\hline $\mathrm{C}^{\prime}{ }^{\prime}$ & $0.50863(8)$ & $0.4587(3)$ & 0.38117 (6) & $0.0741(5)$ \\
\hline $\mathrm{C} 2 \mathrm{~B}$ & $0.43581(7)$ & $0.3946(3)$ & $0.21394(7)$ & $0.0730(5)$ \\
\hline $\mathrm{C}^{\prime}$ & $0.48105(7)$ & $0.2277(3)$ & $0.33438(6)$ & $0.0734(5)$ \\
\hline $\mathrm{C}^{\prime}$ & $0.50283(8)$ & $0.1381(3)$ & $0.40091(7)$ & 0.0735 (5) \\
\hline C6B & $0.30779(8)$ & $0.7433(4)$ & $0.17856(6)$ & $0.0731(5)$ \\
\hline C5B & $0.36357(8)$ & $0.7879(3)$ & $0.17681(7)$ & $0.0741(5)$ \\
\hline $\mathrm{C} 5 \mathrm{~A}$ & $0.63229(9)$ & $-0.1684(3)$ & $0.56795(7)$ & 0.0777 (6) \\
\hline C13B & $0.17693(8)$ & $0.8428(4)$ & $0.27569(7)$ & 0.0797 (6) \\
\hline C13A & $0.82152(8)$ & $-0.0597(3)$ & $0.47209(8)$ & $0.0801(6)$ \\
\hline $\mathrm{C} 10 \mathrm{~A}$ & $0.74957(10)$ & $0.0442(3)$ & $0.41755(6)$ & $0.0790(6)$ \\
\hline $\mathrm{C}^{\prime}$ & $0.48680(8)$ & $0.0933(3)$ & $0.36331(7)$ & $0.0780(5)$ \\
\hline $\mathrm{C} 10 \mathrm{~B}$ & $0.24940(10)$ & $0.9079(3)$ & $0.33226(6)$ & $0.0824(6)$ \\
\hline C6A & $0.68875(9)$ & $-0.1410(5)$ & $0.56883(6)$ & $0.0838(7)$ \\
\hline C11B & $0.19922(10)$ & $0.9302(4)$ & $0.34139(7)$ & $0.0853(6)$ \\
\hline O1B & $0.40194(8)$ & $0.2250(4)$ & 0.14505 (9) & $0.1469(10)$ \\
\hline C11A & $0.79998(11)$ & $0.0491(3)$ & $0.40780(8)$ & $0.0891(7)$ \\
\hline $\mathrm{C} 12 \mathrm{~A}$ & $0.83596(10)$ & $-0.0054(4)$ & $0.43460(8)$ & $0.0919(7)$ \\
\hline C4B & $0.41432(9)$ & $0.8429(4)$ & $0.25758(8)$ & 0.0914 (7) \\
\hline H4BA & 0.436862 & 0.935305 & 0.246868 & $0.137 *$ \\
\hline H4BB & 0.424152 & 0.814614 & 0.284228 & $0.137^{*}$ \\
\hline H4BC & 0.380182 & 0.890062 & 0.257375 & $0.137 *$ \\
\hline $\mathrm{C} 4 \mathrm{~A}$ & $0.58372(10)$ & $-0.1713(4)$ & $0.48615(8)$ & $0.0960(7)$ \\
\hline H4AA & 0.617788 & -0.218047 & 0.484027 & $0.144^{*}$ \\
\hline $\mathrm{H} 4 \mathrm{AB}$ & 0.572169 & -0.131042 & 0.460625 & $0.144^{*}$ \\
\hline $\mathrm{H} 4 \mathrm{AC}$ & 0.561838 & -0.268338 & 0.495797 & $0.144^{*}$ \\
\hline C12B & $0.16312(10)$ & $0.8963(4)$ & $0.31324(8)$ & 0.0908 (7) \\
\hline H3' & $0.4671(5)$ & 0.1999 (19) & $0.3050(4)$ & $0.036(3)^{*}$ \\
\hline H5' & $0.5072(7)$ & $0.034(3)$ & $0.4230(5)$ & $0.058(5)^{*}$ \\
\hline H6BA & $0.2885(8)$ & $0.827(3)$ & $0.1608(7)$ & $0.077(6)^{*}$ \\
\hline $\mathrm{H} 2 \mathrm{~A}$ & $0.5542(9)$ & $0.370(4)$ & $0.5409(7)$ & $0.081(7)^{*}$ \\
\hline H9A & $0.6979(8)$ & -0.011 & $0.4627(6)$ & $0.075(6)^{*}$ \\
\hline $\mathrm{H} 1^{\prime} \mathrm{A}$ & $0.5167(8)$ & $0.593(3)$ & $0.3891(7)$ & $0.081(6)^{*}$ \\
\hline $\mathrm{H} 13 \mathrm{~A}$ & $0.8432(9)$ & $-0.106(3)$ & $0.4913(7)$ & $0.082(7)^{*}$ \\
\hline $\mathrm{H} 4^{\prime}$ & $0.4801(9)$ & -0.041 & $0.3582(7)$ & $0.090(7)^{*}$ \\
\hline H5AA & $0.6207(10)$ & $-0.178(4)$ & $0.5943(9)$ & $0.100(8)^{*}$ \\
\hline $\mathrm{H} 2 \mathrm{~B}$ & $0.4502(9)$ & $0.278(4)$ & $0.2145(7)$ & $0.093(7)^{*}$ \\
\hline H9B & $0.3015(9)$ & $0.846(3)$ & $0.2874(7)$ & $0.090(7)^{*}$ \\
\hline H6BB & $0.2999(9)$ & $0.602(4)$ & $0.1714(7)$ & $0.092(7)^{*}$ \\
\hline H6AA & $0.7045(10)$ & $-0.250(4)$ & $0.5801(8)$ & $0.103(8)^{*}$ \\
\hline
\end{tabular}




\begin{tabular}{lllll} 
H5BA & $0.3718(9)$ & $0.909(3)$ & $0.1898(7)$ & $0.084(7)^{*}$ \\
H11B & $0.1864(9)$ & $0.985(4)$ & $0.3692(8)$ & $0.098(7)^{*}$ \\
H6AB & $0.6966(11)$ & $-0.033(4)$ & $0.5822(9)$ & $0.103(9)^{*}$ \\
H13B & $0.1530(10)$ & $0.809(4)$ & $0.2561(8)$ & $0.105(8)^{*}$ \\
H5BB & $0.3746(9)$ & $0.788(3)$ & $0.1508(8)$ & $0.093(7)^{*}$ \\
H5AB & $0.6241(9)$ & $-0.280(4)$ & $0.5517(7)$ & $0.086(7)^{*}$ \\
H11A & $0.8124(10)$ & $0.095(4)$ & $0.3826(9)$ & $0.110(9)^{*}$ \\
H10A & $0.7236(10)$ & $0.082(4)$ & $0.3987(8)$ & $0.103(8)^{*}$ \\
H10B & $0.2797(12)$ & $0.934(4)$ & $0.3530(9)$ & $0.127(10)^{*}$ \\
H12B & $0.1301(11)$ & $0.918(4)$ & $0.3203(8)$ & $0.109(9)^{*}$ \\
H12A & $0.8730(12)$ & $-0.002(4)$ & $0.4288(9)$ & $0.124(10)^{*}$ \\
H1' & $0.4698(13)$ & $0.507(5)$ & $0.2907(11)$ & $0.145(12)^{*}$ \\
H2' & $0.5357(15)$ & $0.266(6)$ & $0.4639(12)$ & $0.174(15)^{*}$ \\
\hline
\end{tabular}

Atomic displacement parameters $\left(\AA^{2}\right)$

\begin{tabular}{|c|c|c|c|c|c|c|}
\hline & $U^{11}$ & $U^{22}$ & $U^{33}$ & $U^{12}$ & $U^{13}$ & $U^{23}$ \\
\hline O3B & $0.0638(7)$ & $0.0860(9)$ & $0.0530(6)$ & $0.0111(6)$ & $-0.0044(5)$ & $0.0048(6)$ \\
\hline $\mathrm{O} 3 \mathrm{~A}$ & $0.0637(7)$ & $0.0888(9)$ & $0.0549(7)$ & $0.0131(6)$ & $-0.0043(5)$ & $0.0036(6)$ \\
\hline N1A & $0.0560(7)$ & $0.0656(8)$ & $0.0567(8)$ & $0.0012(6)$ & $0.0005(6)$ & $0.0059(7)$ \\
\hline N1B & $0.0565(8)$ & $0.0702(9)$ & $0.0550(8)$ & $-0.0013(7)$ & $0.0003(6)$ & $0.0026(7)$ \\
\hline O4B & $0.0744(9)$ & $0.1351(14)$ & $0.0744(9)$ & $0.0050(9)$ & $-0.0176(7)$ & $-0.0187(9)$ \\
\hline $\mathrm{N} 2 \mathrm{~A}$ & $0.0701(9)$ & $0.0748(10)$ & $0.0646(9)$ & $0.0050(8)$ & $-0.0072(7)$ & $0.0050(8)$ \\
\hline $\mathrm{O} 2^{\prime}$ & $0.1317(14)$ & $0.0893(11)$ & $0.0724(9)$ & $0.0065(10)$ & $-0.0237(9)$ & $0.0092(8)$ \\
\hline O4A & $0.0755(9)$ & $0.1502(16)$ & $0.0777(10)$ & $0.0214(10)$ & $-0.0196(8)$ & $0.0123(10)$ \\
\hline $\mathrm{N} 2 \mathrm{~B}$ & $0.0614(8)$ & $0.0855(11)$ & $0.0694(9)$ & $-0.0011(8)$ & $-0.0056(7)$ & $0.0111(8)$ \\
\hline $\mathrm{O} 1^{\prime}$ & $0.1253(14)$ & $0.1048(12)$ & $0.0845(10)$ & $-0.0311(10)$ & $-0.0359(10)$ & $0.0347(9)$ \\
\hline $\mathrm{O} 2 \mathrm{~A}$ & $0.0989(12)$ & $0.1692(19)$ & $0.0649(9)$ & $0.0149(12)$ & $-0.0146(8)$ & $-0.0071(11)$ \\
\hline N3A & $0.0617(9)$ & $0.1170(16)$ & $0.0789(11)$ & $0.0060(10)$ & $-0.0095(8)$ & $-0.0261(12)$ \\
\hline $\mathrm{C} 8 \mathrm{~B}$ & $0.0630(9)$ & $0.0559(9)$ & $0.0620(9)$ & $0.0073(7)$ & $-0.0047(7)$ & $0.0082(8)$ \\
\hline $\mathrm{O} 2 \mathrm{~B}$ & $0.1083(13)$ & $0.177(2)$ & $0.0778(10)$ & $0.0200(14)$ & $-0.0198(10)$ & $-0.0295(12)$ \\
\hline N3B & $0.0644(10)$ & $0.1138(16)$ & $0.0974(14)$ & $0.0070(10)$ & $-0.0029(10)$ & $-0.0408(13)$ \\
\hline C8A & $0.0653(10)$ & $0.0546(9)$ & $0.0650(10)$ & $0.0009(7)$ & $-0.0032(8)$ & $-0.0134(8)$ \\
\hline $\mathrm{C} 3 \mathrm{~A}$ & $0.0531(9)$ & $0.0731(11)$ & $0.0626(10)$ & $-0.0047(8)$ & $0.0008(7)$ & $-0.0051(8)$ \\
\hline O1A & $0.1041(13)$ & $0.1358(17)$ & $0.1422(18)$ & $0.0224(12)$ & $-0.0328(12)$ & $-0.0735(15)$ \\
\hline C7B & $0.0642(10)$ & $0.0633(10)$ & $0.0637(10)$ & $0.0086(8)$ & $-0.0112(8)$ & $0.0031(8)$ \\
\hline $\mathrm{C} 1 \mathrm{~B}$ & $0.0574(9)$ & $0.0748(11)$ & $0.0578(9)$ & $-0.0063(8)$ & $0.0001(7)$ & $0.0008(8)$ \\
\hline $\mathrm{C} 1 \mathrm{~A}$ & $0.0600(9)$ & $0.0712(11)$ & $0.0631(10)$ & $0.0022(8)$ & $-0.0023(8)$ & $-0.0012(9)$ \\
\hline C7A & $0.0638(10)$ & $0.0679(11)$ & $0.0663(10)$ & $0.0121(8)$ & $-0.0102(8)$ & $-0.0054(9)$ \\
\hline C9A & $0.0735(11)$ & $0.0595(10)$ & $0.0603(10)$ & $0.0014(9)$ & $-0.0032(8)$ & $-0.0057(8)$ \\
\hline $\mathrm{C} 3 \mathrm{~B}$ & $0.0542(9)$ & $0.0754(12)$ & $0.0686(11)$ & $-0.0017(8)$ & $0.0020(8)$ & $-0.0090(9)$ \\
\hline $\mathrm{C} 2 \mathrm{~A}$ & $0.0649(10)$ & 0.0597 (11) & $0.0789(12)$ & $0.0006(9)$ & $-0.0028(9)$ & $0.0007(9)$ \\
\hline $\mathrm{C} 6^{\prime}$ & $0.0618(10)$ & 0.0817 (13) & $0.0626(10)$ & $0.0057(9)$ & $-0.0018(8)$ & $0.0095(9)$ \\
\hline $\mathrm{C} 2^{\prime}$ & $0.0595(10)$ & 0.0903 (14) & $0.0665(11)$ & $-0.0062(9)$ & $-0.0054(8)$ & $0.0188(10)$ \\
\hline C9B & 0.0677 (11) & $0.0771(12)$ & $0.0620(10)$ & $0.0084(9)$ & $-0.0060(8)$ & $0.0044(9)$ \\
\hline $\mathrm{C} 1^{\prime}$ & $0.0758(12)$ & $0.0728(12)$ & $0.0737(12)$ & $-0.0028(10)$ & $-0.0119(9)$ & $0.0096(10)$ \\
\hline $\mathrm{C} 2 \mathrm{~B}$ & $0.0590(10)$ & $0.0697(12)$ & 0.0901 (14) & $0.0022(9)$ & $0.0012(9)$ & $0.0022(11)$ \\
\hline $\mathrm{C} 3^{\prime}$ & $0.0629(10)$ & $0.0871(14)$ & $0.0702(12)$ & $-0.0060(10)$ & $0.0006(9)$ & $0.0015(10)$ \\
\hline
\end{tabular}


supporting information

\begin{tabular}{lllllll} 
C5 $^{\prime}$ & $0.0661(11)$ & $0.0772(13)$ & $0.0771(12)$ & $0.0032(9)$ & $0.0049(9)$ & $0.0168(10)$ \\
C6B & $0.0735(11)$ & $0.0950(15)$ & $0.0507(9)$ & $0.0169(11)$ & $-0.0042(8)$ & $0.0097(10)$ \\
C5B & $0.0786(12)$ & $0.0832(14)$ & $0.0604(11)$ & $0.0058(10)$ & $0.0039(9)$ & $0.0183(10)$ \\
C5A & $0.0805(13)$ & $0.0806(14)$ & $0.0720(13)$ & $0.0147(11)$ & $0.0060(10)$ & $0.0226(11)$ \\
C13B & $0.0654(11)$ & $0.0961(15)$ & $0.0777(13)$ & $0.0082(11)$ & $-0.0049(10)$ & $0.0039(11)$ \\
C13A & $0.0679(11)$ & $0.0853(14)$ & $0.0870(15)$ & $-0.0032(10)$ & $-0.0037(11)$ & $-0.0200(12)$ \\
C10A & $0.1019(15)$ & $0.0701(12)$ & $0.0649(11)$ & $-0.0068(11)$ & $-0.0003(12)$ & $-0.0053(9)$ \\
C4' & $0.0696(12)$ & $0.0771(13)$ & $0.0872(14)$ & $-0.0044(10)$ & $0.0064(10)$ & $0.0008(11)$ \\
C10B & $0.0908(14)$ & $0.0944(15)$ & $0.0621(11)$ & $0.0141(12)$ & $-0.0076(11)$ & $0.0014(10)$ \\
C6A & $0.0753(13)$ & $0.118(2)$ & $0.0580(11)$ & $0.0275(13)$ & $0.0007(9)$ & $0.0181(13)$ \\
C11B & $0.0988(16)$ & $0.0913(15)$ & $0.0657(12)$ & $0.0218(12)$ & $0.0088(11)$ & $0.0113(11)$ \\
O1B & $0.1119(15)$ & $0.1435(19)$ & $0.185(2)$ & $0.0282(14)$ & $-0.0336(15)$ & $-0.0900(18)$ \\
C11A & $0.1141(19)$ & $0.0790(14)$ & $0.0743(14)$ & $-0.0256(13)$ & $0.0189(14)$ & $-0.0207(11)$ \\
C12A & $0.0846(15)$ & $0.0949(16)$ & $0.0961(17)$ & $-0.0210(13)$ & $0.0233(14)$ & $-0.0306(14)$ \\
C4B & $0.0849(14)$ & $0.0990(16)$ & $0.0902(15)$ & $-0.0038(12)$ & $-0.0086(12)$ & $-0.0245(13)$ \\
C4A & $0.0959(16)$ & $0.0983(17)$ & $0.0939(16)$ & $0.0162(13)$ & $-0.0162(13)$ & $-0.0305(14)$ \\
C12B & $0.0744(13)$ & $0.1126(19)$ & $0.0853(15)$ & $0.0143(13)$ & $0.0163(12)$ & $0.0081(13)$ \\
& & & & & & \\
\hline
\end{tabular}

Geometric parameters (A, $\stackrel{\circ}{)}$

\begin{tabular}{llll}
\hline $\mathrm{O} 3 \mathrm{~B}-\mathrm{C} 7 \mathrm{~B}$ & $1.344(2)$ & $\mathrm{N} 3 \mathrm{~B}-\mathrm{C} 3 \mathrm{~B}$ & $1.419(3)$ \\
$\mathrm{O} 3 \mathrm{~B}-\mathrm{C} 6 \mathrm{~B}$ & $1.444(2)$ & $\mathrm{N} 3 \mathrm{~B}-\mathrm{O} 1 \mathrm{~B}$ & $1.208(3)$ \\
$\mathrm{O} 3 \mathrm{~A}-\mathrm{C} 7 \mathrm{~A}$ & $1.345(2)$ & $\mathrm{C} 8 \mathrm{~A}-\mathrm{C} 7 \mathrm{~A}$ & $1.476(3)$ \\
$\mathrm{O} 3 \mathrm{~A}-\mathrm{C} 6 \mathrm{~A}$ & $1.436(2)$ & $\mathrm{C} 8 \mathrm{~A}-\mathrm{C} 9 \mathrm{~A}$ & $1.386(3)$ \\
$\mathrm{N} 1 \mathrm{~A}-\mathrm{C} 3 \mathrm{~A}$ & $1.381(2)$ & $\mathrm{C} 8 \mathrm{~A}-\mathrm{C} 13 \mathrm{~A}$ & $1.392(3)$ \\
$\mathrm{N} 1 \mathrm{~A}-\mathrm{C} 1 \mathrm{~A}$ & $1.356(2)$ & $\mathrm{C} 3 \mathrm{~A}-\mathrm{C} 2 \mathrm{~A}$ & $1.344(3)$ \\
$\mathrm{N} 1 \mathrm{~A}-\mathrm{C} 5 \mathrm{~A}$ & $1.468(2)$ & $\mathrm{C} 1 \mathrm{~B}-\mathrm{C} 4 \mathrm{~B}$ & $1.485(3)$ \\
$\mathrm{N} 1 \mathrm{~B}-\mathrm{C} 1 \mathrm{~B}$ & $1.356(2)$ & $\mathrm{C} 1 \mathrm{~A}-\mathrm{C} 4 \mathrm{~A}$ & $1.480(3)$ \\
$\mathrm{N} 1 \mathrm{~B}-\mathrm{C} 3 \mathrm{~B}$ & $1.379(3)$ & $\mathrm{C} 9 \mathrm{~A}-\mathrm{C} 10 \mathrm{~A}$ & $1.380(3)$ \\
$\mathrm{N} 1 \mathrm{~B}-\mathrm{C} 5 \mathrm{~B}$ & $1.462(3)$ & $\mathrm{C} 3 \mathrm{~B}-\mathrm{C} 2 \mathrm{~B}$ & $1.344(3)$ \\
$\mathrm{O} 4 \mathrm{~B}-\mathrm{C} 7 \mathrm{~B}$ & $1.201(2)$ & $\mathrm{C} 6^{\prime}-\mathrm{C} 1^{\prime}$ & $1.378(3)$ \\
$\mathrm{N} 2 \mathrm{~A}-\mathrm{C} 1 \mathrm{~A}$ & $1.321(3)$ & $\mathrm{C} 6^{\prime}-\mathrm{C} 5^{\prime}$ & $1.382(3)$ \\
$\mathrm{N} 2 \mathrm{~A}-\mathrm{C} 2 \mathrm{~A}$ & $1.348(3)$ & $\mathrm{C} 2^{\prime}-\mathrm{C} 1^{\prime}$ & $1.390(3)$ \\
$\mathrm{O} 2^{\prime}-\mathrm{C} 6^{\prime}$ & $1.367(3)$ & $\mathrm{C} 2^{\prime}-\mathrm{C} 3^{\prime}$ & $1.376(3)$ \\
$\mathrm{O} 4 \mathrm{~A}-\mathrm{C} 7 \mathrm{~A}$ & $1.201(2)$ & $\mathrm{C} 9 \mathrm{~B}-\mathrm{C} 10 \mathrm{~B}$ & $1.381(3)$ \\
$\mathrm{N} 2 \mathrm{~B}-\mathrm{C} 1 \mathrm{~B}$ & $1.327(3)$ & $\mathrm{C} 3^{\prime}-\mathrm{C} 4^{\prime}$ & $1.381(3)$ \\
$\mathrm{N} 2 \mathrm{~B}-\mathrm{C} 2 \mathrm{~B}$ & $1.350(3)$ & $\mathrm{C} 5^{\prime}-\mathrm{C} 4^{\prime}$ & $1.378(3)$ \\
$\mathrm{O} 1^{\prime}-\mathrm{C} 2^{\prime}$ & $1.366(3)$ & $\mathrm{C} 6 \mathrm{~B}-\mathrm{C} 5 \mathrm{~B}$ & $1.504(3)$ \\
$\mathrm{O} 2 \mathrm{~A}-\mathrm{N} 3 \mathrm{~A}$ & $1.223(3)$ & $\mathrm{C} 5 \mathrm{~A}-\mathrm{C} 6 \mathrm{~A}$ & $1.500(3)$ \\
$\mathrm{N} 3 \mathrm{~A}-\mathrm{C} 3 \mathrm{~A}$ & $1.417(3)$ & $\mathrm{C} 13 \mathrm{~B}-\mathrm{C} 12 \mathrm{~B}$ & $1.376(3)$ \\
$\mathrm{N} 3 \mathrm{~A}-\mathrm{O} 1 \mathrm{~A}$ & $1.229(3)$ & $\mathrm{C} 13 \mathrm{~A}-\mathrm{C} 12 \mathrm{~A}$ & $1.380(4)$ \\
$\mathrm{C} 8 \mathrm{~B}-\mathrm{C} 7 \mathrm{~B}$ & $1.478(3)$ & $\mathrm{C} 10 \mathrm{~A}-\mathrm{C} 11 \mathrm{~A}$ & $1.368(4)$ \\
$\mathrm{C} 8 \mathrm{~B}-\mathrm{C} 9 \mathrm{~B}$ & $1.385(3)$ & $\mathrm{C} 10 \mathrm{~B}-\mathrm{C} 11 \mathrm{~B}$ & $1.366(3)$ \\
$\mathrm{C} 8 \mathrm{~B}-\mathrm{C} 13 \mathrm{~B}$ & $1.384(3)$ & $\mathrm{C} 11 \mathrm{~B}-\mathrm{C} 12 \mathrm{~B}$ & $1.367(4)$ \\
$\mathrm{O} 2 \mathrm{~B}-\mathrm{N} 3 \mathrm{~B}$ & $1.218(3)$ & $\mathrm{C} 11 \mathrm{~A}-\mathrm{C} 12 \mathrm{~A}$ & $1.368(4)$ \\
$\mathrm{C} 7 \mathrm{~B}-\mathrm{O} 3 \mathrm{~B}-\mathrm{C} 6 \mathrm{~B}$ & & & $123.95(18)$ \\
$\mathrm{C} 7 \mathrm{~A}-\mathrm{O} 3 \mathrm{~A}-\mathrm{C} 6 \mathrm{~A}$ & $115.49(14)$ & $\mathrm{N} 2 \mathrm{~A}-\mathrm{C} 1 \mathrm{~A}-\mathrm{C} 4 \mathrm{~A}-\mathrm{C} 7 \mathrm{~A}-\mathrm{C} 8 \mathrm{~A}$ & $112.42(15)$
\end{tabular}




$\begin{array}{ll}\mathrm{C} 3 \mathrm{~A}-\mathrm{N} 1 \mathrm{~A}-\mathrm{C} 5 \mathrm{~A} & 129.51(17) \\ \mathrm{C} 1 \mathrm{~A}-\mathrm{N} 1 \mathrm{~A}-\mathrm{C} 3 \mathrm{~A} & 104.96(15) \\ \mathrm{C} 1 \mathrm{~A}-\mathrm{N} 1 \mathrm{~A}-\mathrm{C} 5 \mathrm{~A} & 125.52(18) \\ \mathrm{C} 1 \mathrm{~B}-\mathrm{N} 1 \mathrm{~B}-\mathrm{C} 3 \mathrm{~B} & 105.33(15) \\ \mathrm{C} 1 \mathrm{~B}-\mathrm{N} 1 \mathrm{~B}-\mathrm{C} 5 \mathrm{~B} & 126.37(17) \\ \mathrm{C} 3 \mathrm{~B}-\mathrm{N} 1 \mathrm{~B}-\mathrm{C} 5 \mathrm{~B} & 128.30(17) \\ \mathrm{C} 1 \mathrm{~A}-\mathrm{N} 2 \mathrm{~A}-\mathrm{C} 2 \mathrm{~A} & 106.47(16) \\ \mathrm{C} 1 \mathrm{~B}-\mathrm{N} 2 \mathrm{~B}-\mathrm{C} 2 \mathrm{~B} & 106.10(16) \\ \mathrm{O} 2 \mathrm{~A}-\mathrm{N} 3 \mathrm{~A}-\mathrm{C} 3 \mathrm{~A} & 119.2(2) \\ \mathrm{O} 2 \mathrm{~A}-\mathrm{N} 3 \mathrm{~A}-\mathrm{O} 1 \mathrm{~A} & 124.6(2) \\ \mathrm{O} 1 \mathrm{~A}-\mathrm{N} 3 \mathrm{~A}-\mathrm{C} 3 \mathrm{~A} & 116.2(2) \\ \mathrm{C} 9 \mathrm{~B}-\mathrm{C} 8 \mathrm{~B}-\mathrm{C} 7 \mathrm{~B} & 122.23(17) \\ \mathrm{C} 13 \mathrm{~B}-\mathrm{C} 8 \mathrm{~B}-\mathrm{C} 7 \mathrm{~B} & 118.84(17) \\ \mathrm{C} 13 \mathrm{~B}-\mathrm{C} 8 \mathrm{~B}-\mathrm{C} 9 \mathrm{~B} & 118.91(19) \\ \mathrm{O} 2 \mathrm{~B}-\mathrm{N} 3 \mathrm{~B}-\mathrm{C} 3 \mathrm{~B} & 120.0(2) \\ \text { O1B-N3B-O2B } & 123.8(2) \\ \text { O1B-N3B-C3B } & 116.2(2) \\ \mathrm{C} 9 \mathrm{~A}-\mathrm{C} 8 \mathrm{~A}-\mathrm{C} 7 \mathrm{~A} & 121.98(17) \\ \mathrm{C} 9 \mathrm{~A}-\mathrm{C} 8 \mathrm{~A}-\mathrm{C} 13 \mathrm{~A} & 118.92(19) \\ \mathrm{C} 13 \mathrm{~A}-\mathrm{C} 8 \mathrm{~A}-\mathrm{C} 7 \mathrm{~A} & 119.07(18) \\ \text { N1A-C3A-N3A } & 125.69(18) \\ \mathrm{C} 2 \mathrm{~A}-\mathrm{C} 3 \mathrm{~A}-\mathrm{N} 1 \mathrm{~A} & 107.63(17) \\ \mathrm{C} 2 \mathrm{~A}-\mathrm{C} 3 \mathrm{~A}-\mathrm{N} 3 \mathrm{~A} & 126.7(2) \\ \text { O3B-C7B-C8B } & 112.35(15) \\ \text { O4B-C7B-O3B } & 122.31(18) \\ \text { O4B-C7B-C8B } & 125.32(18) \\ \text { N1B-C1B-C4B } & 124.64(18) \\ \text { N2B-C1B-N1B } & 111.30(17) \\ \text { N2B-C1B-C4B } & 124.06(18) \\ \text { N1A-C1A-C4A } & 124.57(18) \\ \text { N2A-C1A-N1A } & 111.47(17) \\ & \end{array}$

$\begin{array}{ll}\mathrm{O} 4 \mathrm{~A}-\mathrm{C} 7 \mathrm{~A}-\mathrm{O} 3 \mathrm{~A} & 122.22(19) \\ \mathrm{O} 4 \mathrm{~A}-\mathrm{C} 7 \mathrm{~A}-\mathrm{C} 8 \mathrm{~A} & 125.35(19) \\ \mathrm{C} 10 \mathrm{~A}-\mathrm{C} 9 \mathrm{~A}-\mathrm{C} 8 \mathrm{~A} & 119.9(2) \\ \mathrm{N} 1 \mathrm{~B}-\mathrm{C} 3 \mathrm{~B}-\mathrm{N} 3 \mathrm{~B} & 125.01(19) \\ \mathrm{C} 2 \mathrm{~B}-\mathrm{C} 3 \mathrm{~B}-\mathrm{N} 1 \mathrm{~B} & 107.44(18) \\ \mathrm{C} 2 \mathrm{~B}-\mathrm{C} 3 \mathrm{~B}-\mathrm{N} 3 \mathrm{~B} & 127.5(2) \\ \mathrm{C} 3 \mathrm{~A}-\mathrm{C} 2 \mathrm{~A}-\mathrm{N} 2 \mathrm{~A} & 109.46(18) \\ \mathrm{O} 2^{\prime}-\mathrm{C} 6^{\prime}-\mathrm{C} 1^{\prime} & 116.5(2) \\ \mathrm{O} 2^{\prime}-\mathrm{C} 6^{\prime}-\mathrm{C} 5^{\prime} & 123.01(18) \\ \mathrm{C} 1^{\prime}-\mathrm{C} 6^{\prime}-\mathrm{C} 5^{\prime} & 120.5(2) \\ \mathrm{O} 1^{\prime}-\mathrm{C} 2^{\prime}-\mathrm{C} 1^{\prime} & 116.9(2) \\ \mathrm{O} 1^{\prime}-\mathrm{C} 2^{\prime}-\mathrm{C} 3^{\prime} & 122.55(19) \\ \mathrm{C} 3^{\prime}-\mathrm{C} 2^{\prime}-\mathrm{C} 1^{\prime} & 120.55(19) \\ \mathrm{C} 10 \mathrm{~B}-\mathrm{C} 9 \mathrm{~B}-\mathrm{C} 8 \mathrm{~B} & 119.90(19) \\ \mathrm{C} 6^{\prime}-\mathrm{C} 1^{\prime}-\mathrm{C} 2^{\prime} & 119.7(2) \\ \mathrm{C} 3 \mathrm{~B}-\mathrm{C} 2 \mathrm{~B}-\mathrm{N} 2 \mathrm{~B} & 109.83(19) \\ \mathrm{C} 2^{\prime}-\mathrm{C} 3^{\prime}-\mathrm{C} 4^{\prime} & 118.7(2) \\ \mathrm{C} 4^{\prime}-\mathrm{C} 5^{\prime}-\mathrm{C} 6^{\prime} & 118.9(2) \\ \mathrm{O} 3 \mathrm{~B}-\mathrm{C} 6 \mathrm{~B}-\mathrm{C} 5 \mathrm{~B} & 106.70(17) \\ \mathrm{N} 1 \mathrm{~B}-\mathrm{C} 5 \mathrm{~B}-\mathrm{C} 6 \mathrm{~B} & 112.24(17) \\ \mathrm{N} 1 \mathrm{~A}-\mathrm{C} 5 \mathrm{~A}-\mathrm{C} 6 \mathrm{~A} & 113.3(2) \\ \mathrm{C} 12 \mathrm{~B}-\mathrm{C} 13 \mathrm{~B}-\mathrm{C} 8 \mathrm{~B} & 120.2(2) \\ \mathrm{C} 12 \mathrm{~A}-\mathrm{C} 13 \mathrm{~A}-\mathrm{C} 8 \mathrm{~A} & 120.3(2) \\ \mathrm{C} 11 \mathrm{~A}-\mathrm{C} 10 \mathrm{~A}-\mathrm{C} 9 \mathrm{~A} & 120.6(2) \\ \mathrm{C} 5^{\prime}-\mathrm{C} 4^{\prime}-\mathrm{C} 3^{\prime} & 121.7(2) \\ \mathrm{C} 11 \mathrm{~B}-\mathrm{C} 10 \mathrm{~B}-\mathrm{C} 9 \mathrm{~B} & 120.7(2) \\ \mathrm{O} 3 \mathrm{~A}-\mathrm{C} 6 \mathrm{~A}-\mathrm{C} 5 \mathrm{~A} & 107.72(18) \\ \mathrm{C} 10 \mathrm{~B}-\mathrm{C} 11 \mathrm{~B}-\mathrm{C} 12 \mathrm{~B} & 119.6(2) \\ \mathrm{C} 10 \mathrm{~A}-\mathrm{C} 11 \mathrm{~A}-\mathrm{C} 12 \mathrm{~A} & 120.3(2) \\ \mathrm{C} 11 \mathrm{~A}-\mathrm{C} 12 \mathrm{~A}-\mathrm{C} 13 \mathrm{~A} & 119.9(2) \\ \mathrm{C} 11 \mathrm{~B}-\mathrm{C} 12 \mathrm{~B}-\mathrm{C} 13 \mathrm{~B} & 120.6(2) \\ & \end{array}$

2-(2-Methyl-5-nitro-1 $H$-imidazol-1-yl)ethyl benzoate-fumaric acid (2/1) (bzmdfma)

Crystal data

$\mathrm{C}_{13} \mathrm{H}_{13} \mathrm{~N}_{3} \mathrm{O}_{4} \cdot 0.5 \mathrm{C}_{4} \mathrm{H}_{4} \mathrm{O}_{4}$

$M_{r}=333.30$

Monoclinic, $P 2_{1} / c$

$a=9.0358(5) \AA$

$b=26.6419(14) \AA$

$c=6.8796(3) \AA$

$\beta=102.419(2)^{\circ}$

$V=1617.38(14) \AA^{3}$

$Z=4$

Data collection

Bruker APEXII CCD

diffractometer

$\varphi$ and $\omega$ scans
$F(000)=696$

$D_{\mathrm{x}}=1.369 \mathrm{Mg} \mathrm{m}^{-3}$

Mo $K \alpha$ radiation, $\lambda=0.71073 \AA$

Cell parameters from 9988 reflections

$\theta=2.3-28.9^{\circ}$

$\mu=0.11 \mathrm{~mm}^{-1}$

$T=300 \mathrm{~K}$

Prism, clear light colourless

$0.63 \times 0.17 \times 0.17 \mathrm{~mm}$ 
3705 independent reflections

2625 reflections with $I>2 \sigma(I)$

$R_{\text {int }}=0.066$

$\theta_{\max }=27.5^{\circ}, \theta_{\min }=2.8^{\circ}$

\section{Refinement}

Refinement on $F^{2}$

Least-squares matrix: full

$R\left[F^{2}>2 \sigma\left(F^{2}\right)\right]=0.051$

$w R\left(F^{2}\right)=0.151$

$S=1.05$

3705 reflections

267 parameters

0 restraints

Primary atom site location: dual

Hydrogen site location: mixed

$$
\begin{aligned}
& h=-11 \rightarrow 11 \\
& k=-34 \rightarrow 34 \\
& l=-8 \rightarrow 8
\end{aligned}
$$

Special details

$\mathrm{H}$ atoms treated by a mixture of independent and constrained refinement

$w=1 /\left[\sigma^{2}\left(F_{\mathrm{o}}^{2}\right)+(0.0568 P)^{2}+0.5999 P\right]$ where $P=\left(F_{\mathrm{o}}^{2}+2 F_{\mathrm{c}}^{2}\right) / 3$

$(\Delta / \sigma)_{\max }<0.001$

$\Delta \rho_{\max }=0.39 \mathrm{e} \AA^{-3}$

$\Delta \rho_{\min }=-0.33$ e $\AA^{-3}$

Extinction correction: SHELXL2018

(Sheldrick, 2015b),

$\mathrm{Fc}^{*}=\mathrm{kFc}\left[1+0.001 \mathrm{xFc}^{2} \lambda^{3} / \sin (2 \theta)\right]^{-1 / 4}$

Extinction coefficient: $0.018(3)$

Geometry. All esds (except the esd in the dihedral angle between two 1.s. planes) are estimated using the full covariance matrix. The cell esds are taken into account individually in the estimation of esds in distances, angles and torsion angles; correlations between esds in cell parameters are only used when they are defined by crystal symmetry. An approximate (isotropic) treatment of cell esds is used for estimating esds involving l.s. planes.

Refinement. Single-crystal X-ray diffraction data collection ( scans and $\omega$ scans with $\kappa$ and $\theta$ offsets) were performed at room temperature on a Bruker AXS D8 VENTURE equipped with a Kappa goniometer, PHOTON II CPAD detector, a Mo $K \alpha$ INCOATEC I $\mu \mathrm{S} 3.0$ microfocus source $(\lambda=0.71073 \AA)$. The crystal centring, unit-cell determination, refinement of the unit-cell parameters and data collection was controlled through the program APEX3 (Bruker, 2012). The frame integration was performed using SAINT (Bruker, 2016) and the intensities were scaled and absorption corrected using SADABS (Bruker, 2001). Using OLEX2 (Dolomanov et al., 2009), the structure was solved by intrinsic phasing using SHELXT (Sheldrick, 2015) and refined by full-matrix least-squares calculation based on $F^{2}$ for all reflection using SHELXL (Sheldrick, 2007). All non-H atoms were refined anisotropically.

Fractional atomic coordinates and isotropic or equivalent isotropic displacement parameters $\left(\hat{A}^{2}\right)$

\begin{tabular}{lllll}
\hline & $x$ & $y$ & $z$ & $U_{\text {iso }} * / U_{\text {eq }}$ \\
\hline O1 & $0.8887(3)$ & $0.59652(9)$ & $0.3299(4)$ & $0.1261(9)$ \\
O2 & $0.9417(2)$ & $0.62892(9)$ & $0.6242(4)$ & $0.1134(8)$ \\
O3 & $0.55707(16)$ & $0.70919(5)$ & $0.7641(2)$ & $0.0614(4)$ \\
O4 & $0.6408(2)$ & $0.78808(6)$ & $0.7663(3)$ & $0.0818(5)$ \\
N1 & $0.64502(18)$ & $0.60717(6)$ & $0.6676(2)$ & $0.0535(4)$ \\
N2 & $0.4807(2)$ & $0.56134(6)$ & $0.4570(3)$ & $0.0640(5)$ \\
N3 & $0.8562(3)$ & $0.60697(8)$ & $0.4893(4)$ & $0.0820(6)$ \\
C1 & $0.5053(2)$ & $0.58656(7)$ & $0.6269(3)$ & $0.0574(5)$ \\
C2 & $0.6064(3)$ & $0.56549(8)$ & $0.3829(4)$ & $0.0649(6)$ \\
H2 & $0.620(3)$ & $0.5508(9)$ & $0.262(4)$ & $0.078(7)^{*}$ \\
C3 & $0.7094(2)$ & $0.59375(7)$ & $0.5099(3)$ & $0.0575(5)$ \\
C4 & $0.3945(3)$ & $0.59055(10)$ & $0.7556(4)$ & $0.0838(7)$ \\
H4A & 0.417788 & 0.566096 & 0.860183 & $0.126^{*}$ \\
H4B & 0.294408 & 0.584567 & 0.677807 & $0.126^{*}$ \\
H4C & 0.399275 & 0.623576 & 0.812368 & $0.126^{*}$ \\
C5 & $0.7116(3)$ & $0.63718(9)$ & $0.8440(3)$ & $0.0633(5)$ \\
H5A & $0.650(3)$ & $0.6299(9)$ & $0.940(4)$ & $0.069(6)^{*}$
\end{tabular}




$\begin{array}{lllll}\text { H5B } & 0.815(3) & 0.6255(9) & 0.896(3) & 0.073(7)^{*} \\ \text { C6 } & 0.7118(2) & 0.69224(9) & 0.7997(4) & 0.0651(6) \\ \text { H6A } & 0.766(3) & 0.7091(9) & 0.907(4) & 0.073(7)^{*} \\ \text { H6B } & 0.751(3) & 0.6996(9) & 0.672(4) & 0.075(7)^{*} \\ \text { C7 } & 0.5365(2) & 0.75911(8) & 0.7537(3) & 0.0564(5) \\ \text { C8 } & 0.3754(2) & 0.77333(7) & 0.7313(3) & 0.0540(5) \\ \text { C9 } & 0.2606(3) & 0.73811(9) & 0.7113(3) & 0.0605(5) \\ \text { H9 } & 0.287(3) & 0.7036(10) & 0.710(4) & 0.082(8)^{*} \\ \text { C10 } & 0.1132(3) & 0.75312(10) & 0.6989(4) & 0.0725(6) \\ \text { H10 } & 0.031(3) & 0.7287(10) & 0.685(4) & 0.092(8)^{*} \\ \text { C11 } & 0.0794(3) & 0.80314(11) & 0.7083(4) & 0.0807(7) \\ \text { H11 } & -0.026(3) & 0.8135(10) & 0.702(4) & 0.087(8)^{*} \\ \text { C12 } & 0.1916(4) & 0.83847(11) & 0.7265(4) & 0.0856(8) \\ \text { H12 } & 0.163(3) & 0.8738(12) & 0.734(4) & 0.101(9)^{*} \\ \text { C13 } & 0.3391(3) & 0.82380(9) & 0.7366(4) & 0.0717(6) \\ \text { H13 } & 0.421(3) & 0.8472(11) & 0.743(4) & 0.094(9)^{*} \\ \text { O1' } & 0.21422(19) & 0.52552(7) & 0.2699(3) & 0.0859(6) \\ \text { H1' } & 0.317(4) & 0.5368(13) & 0.342(5) & 0.121(11)^{*} \\ \text { O2 }^{\prime} & 0.32943(17) & 0.49184(7) & 0.0484(3) & 0.0836(5) \\ \text { C1' } & 0.2163(2) & 0.50351(7) & 0.1006(3) & 0.0578(5) \\ \text { C2' } & 0.0625(2) & 0.49372(8) & -0.0245(4) & 0.0629(5) \\ \text { H2 }^{\prime} & 0.055(3) & 0.4785(11) & -0.152(5) & 0.102(9)^{*}\end{array}$

Atomic displacement parameters $\left(\AA^{2}\right)$

\begin{tabular}{lllllll}
\hline & $U^{11}$ & $U^{22}$ & $U^{33}$ & $U^{12}$ & $U^{13}$ & $U^{23}$ \\
\hline O1 & $0.1359(19)$ & $0.1154(17)$ & $0.157(2)$ & $-0.0397(14)$ & $0.0970(17)$ & $-0.0559(15)$ \\
O2 & $0.0673(12)$ & $0.1338(18)$ & $0.1422(19)$ & $-0.0313(12)$ & $0.0291(12)$ & $-0.0469(15)$ \\
O3 & $0.0542(8)$ & $0.0556(8)$ & $0.0704(9)$ & $-0.0035(6)$ & $0.0045(6)$ & $-0.0106(6)$ \\
O4 & $0.0761(11)$ & $0.0657(10)$ & $0.1059(13)$ & $-0.0188(8)$ & $0.0246(9)$ & $-0.0086(9)$ \\
N1 & $0.0530(9)$ & $0.0500(9)$ & $0.0554(9)$ & $-0.0022(7)$ & $0.0070(7)$ & $-0.0012(7)$ \\
N2 & $0.0587(11)$ & $0.0481(9)$ & $0.0776(12)$ & $-0.0030(7)$ & $-0.0020(9)$ & $0.0004(8)$ \\
N3 & $0.0767(14)$ & $0.0666(12)$ & $0.1122(17)$ & $-0.0152(10)$ & $0.0416(13)$ & $-0.0255(11)$ \\
C1 & $0.0508(11)$ & $0.0476(10)$ & $0.0712(13)$ & $-0.0010(8)$ & $0.0070(9)$ & $0.0084(9)$ \\
C2 & $0.0772(15)$ & $0.0472(11)$ & $0.0676(13)$ & $-0.0024(10)$ & $0.0094(11)$ & $-0.0061(9)$ \\
C3 & $0.0590(12)$ & $0.0471(10)$ & $0.0667(12)$ & $-0.0040(8)$ & $0.0147(9)$ & $-0.0041(9)$ \\
C4 & $0.0685(15)$ & $0.0803(16)$ & $0.109(2)$ & $-0.0047(12)$ & $0.0329(14)$ & $0.0080(14)$ \\
C5 & $0.0616(13)$ & $0.0701(14)$ & $0.0529(11)$ & $0.0021(10)$ & $0.0008(10)$ & $-0.0056(10)$ \\
C6 & $0.0532(12)$ & $0.0675(13)$ & $0.0686(14)$ & $-0.0051(10)$ & $0.0000(10)$ & $-0.0185(11)$ \\
C7 & $0.0679(13)$ & $0.0558(11)$ & $0.0450(10)$ & $-0.0084(9)$ & $0.0112(9)$ & $-0.0079(8)$ \\
C8 & $0.0648(12)$ & $0.0562(11)$ & $0.0385(9)$ & $-0.0020(9)$ & $0.0058(8)$ & $-0.0047(8)$ \\
C9 & $0.0643(13)$ & $0.0598(13)$ & $0.0535(11)$ & $-0.0015(10)$ & $0.0042(9)$ & $-0.0051(9)$ \\
C10 & $0.0626(14)$ & $0.0813(16)$ & $0.0685(14)$ & $-0.0029(12)$ & $0.0027(11)$ & $-0.0098(12)$ \\
C11 & $0.0717(17)$ & $0.0923(19)$ & $0.0706(15)$ & $0.0176(15)$ & $-0.0017(12)$ & $-0.0112(13)$ \\
C12 & $0.092(2)$ & $0.0672(16)$ & $0.0907(18)$ & $0.0186(14)$ & $0.0038(14)$ & $-0.0066(13)$ \\
C13 & $0.0855(17)$ & $0.0556(13)$ & $0.0709(14)$ & $-0.0021(12)$ & $0.0099(12)$ & $-0.0047(10)$ \\
O1' & $0.0529(10)$ & $0.0983(13)$ & $0.1000(13)$ & $-0.0022(8)$ & $0.0019(8)$ & $-0.0331(10)$ \\
O2' & $0.0461(9)$ & $0.1114(14)$ & $0.0934(12)$ & $0.0015(8)$ & $0.0152(8)$ & $-0.0062(10)$ \\
& & & & & &
\end{tabular}




\begin{tabular}{lllllll}
$\mathrm{C} 1^{\prime}$ & $0.0497(11)$ & $0.0496(11)$ & $0.0720(13)$ & $-0.0020(8)$ & $0.0085(9)$ & $-0.0015(9)$ \\
$\mathrm{C} 2^{\prime}$ & $0.0479(11)$ & $0.0601(12)$ & $0.0785(14)$ & $-0.0023(9)$ & $0.0083(10)$ & $-0.0123(10)$ \\
\hline
\end{tabular}

Geometric parameters $(\AA, \stackrel{o}{)}$

\begin{tabular}{|c|c|c|c|}
\hline $\mathrm{O} 1-\mathrm{N} 3$ & $1.227(3)$ & $\mathrm{C} 6-\mathrm{H} 6 \mathrm{~A}$ & $0.91(3)$ \\
\hline $\mathrm{O} 2-\mathrm{N} 3$ & $1.221(3)$ & C6-H6B & $1.03(3)$ \\
\hline $\mathrm{O} 3-\mathrm{C} 6$ & $1.439(3)$ & $\mathrm{C} 7-\mathrm{C} 8$ & $1.480(3)$ \\
\hline $\mathrm{O} 3-\mathrm{C} 7$ & $1.343(2)$ & $\mathrm{C} 8-\mathrm{C} 9$ & $1.383(3)$ \\
\hline $\mathrm{O} 4-\mathrm{C} 7$ & $1.206(2)$ & $\mathrm{C} 8-\mathrm{C} 13$ & $1.386(3)$ \\
\hline $\mathrm{N} 1-\mathrm{C} 1$ & $1.350(3)$ & C9- $\mathrm{H} 9$ & $0.95(3)$ \\
\hline $\mathrm{N} 1-\mathrm{C} 3$ & $1.384(3)$ & $\mathrm{C} 9-\mathrm{C} 10$ & $1.375(3)$ \\
\hline $\mathrm{N} 1-\mathrm{C} 5$ & $1.469(3)$ & $\mathrm{C} 10-\mathrm{H} 10$ & $0.98(3)$ \\
\hline $\mathrm{N} 2-\mathrm{C} 1$ & $1.325(3)$ & $\mathrm{C} 10-\mathrm{C} 11$ & $1.372(4)$ \\
\hline $\mathrm{N} 2-\mathrm{C} 2$ & $1.346(3)$ & $\mathrm{C} 11-\mathrm{H} 11$ & $0.98(3)$ \\
\hline $\mathrm{N} 3-\mathrm{C} 3$ & $1.408(3)$ & $\mathrm{C} 11-\mathrm{C} 12$ & $1.369(4)$ \\
\hline $\mathrm{C} 1-\mathrm{C} 4$ & $1.475(3)$ & C12-H12 & $0.98(3)$ \\
\hline $\mathrm{C} 2-\mathrm{H} 2$ & $0.95(2)$ & $\mathrm{C} 12-\mathrm{C} 13$ & $1.376(4)$ \\
\hline $\mathrm{C} 2-\mathrm{C} 3$ & $1.359(3)$ & $\mathrm{C} 13-\mathrm{H} 13$ & $0.96(3)$ \\
\hline $\mathrm{C} 4-\mathrm{H} 4 \mathrm{~A}$ & 0.9600 & $\mathrm{O} 1^{\prime}-\mathrm{H} 1^{\prime}$ & $1.00(4)$ \\
\hline $\mathrm{C} 4-\mathrm{H} 4 \mathrm{~B}$ & 0.9600 & $\mathrm{O} 1^{\prime}-\mathrm{C} 1^{\prime}$ & $1.308(3)$ \\
\hline $\mathrm{C} 4-\mathrm{H} 4 \mathrm{C}$ & 0.9600 & $\mathrm{O} 2^{\prime}-\mathrm{C} 1^{\prime}$ & $1.195(2)$ \\
\hline $\mathrm{C} 5-\mathrm{H} 5 \mathrm{~A}$ & $0.97(2)$ & $\mathrm{C} 1^{\prime}-\mathrm{C} 2^{\prime}$ & $1.492(3)$ \\
\hline $\mathrm{C} 5-\mathrm{H} 5 \mathrm{~B}$ & $0.98(3)$ & $\mathrm{C} 2^{\prime}-\mathrm{C} 2^{\prime \mathrm{i}}$ & $1.290(4)$ \\
\hline $\mathrm{C} 5-\mathrm{C} 6$ & $1.498(3)$ & $\mathrm{C} 2^{\prime}-\mathrm{H} 2^{\prime}$ & $0.96(3)$ \\
\hline $\mathrm{C} 7-\mathrm{O} 3-\mathrm{C} 6$ & $115.97(16)$ & $\mathrm{C} 5-\mathrm{C} 6-\mathrm{H} 6 \mathrm{~A}$ & $110.0(15)$ \\
\hline $\mathrm{C} 1-\mathrm{N} 1-\mathrm{C} 3$ & $105.33(17)$ & $\mathrm{C} 5-\mathrm{C} 6-\mathrm{H} 6 \mathrm{~B}$ & $112.0(13)$ \\
\hline $\mathrm{C} 1-\mathrm{N} 1-\mathrm{C} 5$ & $126.25(19)$ & $\mathrm{H} 6 \mathrm{~A}-\mathrm{C} 6-\mathrm{H} 6 \mathrm{~B}$ & $112(2)$ \\
\hline $\mathrm{C} 3-\mathrm{N} 1-\mathrm{C} 5$ & $128.42(18)$ & $\mathrm{O} 3-\mathrm{C} 7-\mathrm{C} 8$ & $112.38(17)$ \\
\hline $\mathrm{C} 1-\mathrm{N} 2-\mathrm{C} 2$ & $107.32(18)$ & $\mathrm{O} 4-\mathrm{C} 7-\mathrm{O} 3$ & $122.2(2)$ \\
\hline $\mathrm{O} 1-\mathrm{N} 3-\mathrm{C} 3$ & $116.4(2)$ & $\mathrm{O} 4-\mathrm{C} 7-\mathrm{C} 8$ & $125.4(2)$ \\
\hline $\mathrm{O} 2-\mathrm{N} 3-\mathrm{O} 1$ & $123.6(2)$ & $\mathrm{C} 9-\mathrm{C} 8-\mathrm{C} 7$ & 122.44 (19) \\
\hline $\mathrm{O} 2-\mathrm{N} 3-\mathrm{C} 3$ & $119.9(2)$ & $\mathrm{C} 9-\mathrm{C} 8-\mathrm{C} 13$ & $118.9(2)$ \\
\hline $\mathrm{N} 1-\mathrm{C} 1-\mathrm{C} 4$ & $124.8(2)$ & $\mathrm{C} 13-\mathrm{C} 8-\mathrm{C} 7$ & $118.6(2)$ \\
\hline $\mathrm{N} 2-\mathrm{C} 1-\mathrm{N} 1$ & 111.17 (19) & $\mathrm{C} 8-\mathrm{C} 9-\mathrm{H} 9$ & $118.1(16)$ \\
\hline $\mathrm{N} 2-\mathrm{C} 1-\mathrm{C} 4$ & $124.0(2)$ & $\mathrm{C} 10-\mathrm{C} 9-\mathrm{C} 8$ & $120.3(2)$ \\
\hline $\mathrm{N} 2-\mathrm{C} 2-\mathrm{H} 2$ & $125.0(15)$ & $\mathrm{C} 10-\mathrm{C} 9-\mathrm{H} 9$ & $121.5(16)$ \\
\hline $\mathrm{N} 2-\mathrm{C} 2-\mathrm{C} 3$ & $108.6(2)$ & $\mathrm{C} 9-\mathrm{C} 10-\mathrm{H} 10$ & $121.3(16)$ \\
\hline $\mathrm{C} 3-\mathrm{C} 2-\mathrm{H} 2$ & $126.4(15)$ & $\mathrm{C} 11-\mathrm{C} 10-\mathrm{C} 9$ & $120.1(3)$ \\
\hline $\mathrm{N} 1-\mathrm{C} 3-\mathrm{N} 3$ & $125.29(19)$ & $\mathrm{C} 11-\mathrm{C} 10-\mathrm{H} 10$ & $118.5(16)$ \\
\hline $\mathrm{C} 2-\mathrm{C} 3-\mathrm{N} 1$ & $107.59(19)$ & $\mathrm{C} 10-\mathrm{C} 11-\mathrm{H} 11$ & 119.7 (16) \\
\hline $\mathrm{C} 2-\mathrm{C} 3-\mathrm{N} 3$ & $127.1(2)$ & $\mathrm{C} 12-\mathrm{C} 11-\mathrm{C} 10$ & $120.3(3)$ \\
\hline $\mathrm{C} 1-\mathrm{C} 4-\mathrm{H} 4 \mathrm{~A}$ & 109.5 & $\mathrm{C} 12-\mathrm{C} 11-\mathrm{H} 11$ & $120.0(16)$ \\
\hline $\mathrm{C} 1-\mathrm{C} 4-\mathrm{H} 4 \mathrm{~B}$ & 109.5 & $\mathrm{C} 11-\mathrm{C} 12-\mathrm{H} 12$ & $117.6(17)$ \\
\hline $\mathrm{C} 1-\mathrm{C} 4-\mathrm{H} 4 \mathrm{C}$ & 109.5 & $\mathrm{C} 11-\mathrm{C} 12-\mathrm{C} 13$ & $120.0(3)$ \\
\hline $\mathrm{H} 4 \mathrm{~A}-\mathrm{C} 4-\mathrm{H} 4 \mathrm{~B}$ & 109.5 & $\mathrm{C} 13-\mathrm{C} 12-\mathrm{H} 12$ & 122.4 (17) \\
\hline $\mathrm{H} 4 \mathrm{~A}-\mathrm{C} 4-\mathrm{H} 4 \mathrm{C}$ & 109.5 & $\mathrm{C} 8-\mathrm{C} 13-\mathrm{H} 13$ & $116.6(17)$ \\
\hline
\end{tabular}




$\begin{array}{llll}\mathrm{H} 4 \mathrm{~B}-\mathrm{C} 4-\mathrm{H} 4 \mathrm{C} & 109.5 & \mathrm{C} 12-\mathrm{C} 13-\mathrm{C} 8 & 120.4(2) \\ \mathrm{N} 1-\mathrm{C} 5-\mathrm{H} 5 \mathrm{~A} & 105.1(14) & \mathrm{C} 12-\mathrm{C} 13-\mathrm{H} 13 & 123.0(17) \\ \mathrm{N} 1-\mathrm{C} 5-\mathrm{H} 5 \mathrm{~B} & 108.7(14) & \mathrm{C} 1^{\prime}-\mathrm{O} 1^{\prime}-\mathrm{H} 1^{\prime} & 112.7(19) \\ \mathrm{N} 1-\mathrm{C} 5-\mathrm{C} 6 & 112.52(18) & \mathrm{O} 1^{\prime}-\mathrm{C} 1^{\prime}-\mathrm{C} 2^{\prime} & 113.71(19) \\ \mathrm{H} 5 \mathrm{~A}-\mathrm{C} 5-\mathrm{H} 5 \mathrm{~B} & 109.2(19) & \mathrm{O} 2^{\prime}-\mathrm{C} 1^{\prime}-\mathrm{O}^{\prime} & 124.1(2) \\ \mathrm{C} 6-\mathrm{C} 5-\mathrm{H} 5 \mathrm{~A} & 111.0(14) & \mathrm{O} 2^{\prime}-\mathrm{C} 1^{\prime}-\mathrm{C} 2^{\prime} & 122.2(2) \\ \mathrm{C} 6-\mathrm{C} 5-\mathrm{H} 5 \mathrm{~B} & 110.2(14) & \mathrm{C} 1^{\prime}-\mathrm{C} 2^{\prime}-\mathrm{H} 2^{\prime} & 118.3(17) \\ \mathrm{O} 3-\mathrm{C} 6-\mathrm{C} 5 & 107.36(19) & \mathrm{C} 2^{\prime \prime}-\mathrm{C} 2^{\prime}-\mathrm{C} 1^{\prime} & 124.3(3) \\ \mathrm{O} 3-\mathrm{C} 6-\mathrm{H} 6 \mathrm{~A} & 108.5(15) & \mathrm{C} 2^{\prime \prime}-\mathrm{C} 2^{\prime}-\mathrm{H} 2^{\prime} & 117.3(17) \\ \mathrm{O} 3-\mathrm{C} 6-\mathrm{H} 6 \mathrm{~B} & 107.2(14) & & \end{array}$

Symmetry code: (i) $-x,-y+1,-z$.

2-(2-Methyl-5-nitro-1H-imidazol-1-yl)ethyl benzoate-malonic acid (2/1) (bzmdmln)

Crystal data

$2 \mathrm{C}_{13} \mathrm{H}_{13} \mathrm{~N}_{3} \mathrm{O}_{4} \cdot \mathrm{C}_{3} \mathrm{H}_{2} \mathrm{O}_{4}$

$M_{r}=652.57$

$D_{\mathrm{x}}=1.367 \mathrm{Mg} \mathrm{m}^{-3}$

Orthorhombic, $\mathrm{Pbcn}$

$\mathrm{Cu} K \alpha$ radiation, $\lambda=1.54178 \AA$

$a=26.1542(4) \AA$

$b=7.2708(1) \AA$

$c=16.6719(3) \AA$

Cell parameters from 9937 reflections

$\theta=3.1-72.5^{\circ}$

$\mu=0.92 \mathrm{~mm}^{-1}$

$V=3170.36(9) \AA^{3}$

$T=300 \mathrm{~K}$

$Z=4$

Prism, clear light colourless

$F(000)=1360$

$0.46 \times 0.29 \times 0.13 \mathrm{~mm}$

Data collection

Bruker APEXII CCD

2893 independent reflections

diffractometer

$\varphi$ and $\omega$ scans

Absorption correction: numerical

(SADABS; Bruker, 2016)

$T_{\min }=0.736, T_{\max }=0.907$

54755 measured reflections 2423 reflections with $I>2 \sigma(I)$

$R_{\text {int }}=0.038$

$\theta_{\text {max }}=68.2^{\circ}, \theta_{\text {min }}=5.6^{\circ}$

$h=-31 \rightarrow 31$

$k=-8 \rightarrow 8$

$l=-20 \rightarrow 20$

\section{Refinement}

Refinement on $F^{2}$

Least-squares matrix: full

$R\left[F^{2}>2 \sigma\left(F^{2}\right)\right]=0.078$

$w R\left(F^{2}\right)=0.268$

$S=1.13$

2893 reflections

244 parameters

36 restraints

Primary atom site location: dual

Hydrogen site location: inferred from

neighbouring sites

$\mathrm{H}$-atom parameters constrained

$w=1 /\left[\sigma^{2}\left(F_{\mathrm{o}}^{2}\right)+(0.1596 P)^{2}+0.3629 P\right]$

where $P=\left(F_{\mathrm{o}}^{2}+2 F_{\mathrm{c}}^{2}\right) / 3$

$(\Delta / \sigma)_{\max }=0.001$

$\Delta \rho_{\max }=0.33$ e $\AA^{-3}$

$\Delta \rho_{\min }=-0.27$ e $\AA^{-3}$

Extinction correction: SHELXL2018

(Sheldrick, 2015b),

$\mathrm{Fc}^{*}=\mathrm{kFc}\left[1+0.001 \mathrm{xFc}^{2} \lambda^{3} / \sin (2 \theta)\right]^{-1 / 4}$

Extinction coefficient: 0.0039 (11)

Special details

Geometry. All esds (except the esd in the dihedral angle between two 1.s. planes) are estimated using the full covariance matrix. The cell esds are taken into account individually in the estimation of esds in distances, angles and torsion angles; correlations between esds in cell parameters are only used when they are defined by crystal symmetry. An approximate (isotropic) treatment of cell esds is used for estimating esds involving l.s. planes. 
Refinement. Single-crystal X-ray diffraction data collection ( scans and $\omega$ scans with $\kappa$ and $\theta$ offsets) were performed at room temperature on a Bruker AXS D8 VENTURE equipped with a Kappa goniometer, PHOTON II CPAD detector, a Mo $K \alpha$ INCOATEC I $\mu$ S 3.0 microfocus source $(\lambda=0.71073 \AA$ ). The crystal centring, unit-cell determination, refinement of the unit-cell parameters and data collection was controlled through the program APEX3 (Bruker, 2012). The frame integration was performed using SAINT (Bruker, 2016) and the intensities were scaled and absorption corrected using SADABS (Bruker, 2001). Using OLEX2 (Dolomanov et al., 2009), the structure was solved by intrinsic phasing using SHELXT (Sheldrick, 2015) and refined by full-matrix least-squares calculation based on $F^{2}$ for all reflection using SHELXL (Sheldrick, 2007). All non-H atoms were refined anisotropically.

Fractional atomic coordinates and isotropic or equivalent isotropic displacement parameters $\left(\AA^{2}\right)$

\begin{tabular}{|c|c|c|c|c|c|}
\hline & $x$ & $y$ & $z$ & $U_{\text {iso }} * / U_{\text {eq }}$ & Occ. $(<1)$ \\
\hline $\mathrm{O} 3$ & $0.29519(7)$ & $0.6661(3)$ & $0.53894(10)$ & $0.1002(6)$ & \\
\hline $\mathrm{O} 4$ & $0.21543(9)$ & $0.6847(4)$ & $0.58610(14)$ & $0.1407(10)$ & \\
\hline N1 & $0.39769(8)$ & $0.5613(3)$ & 0.58207 (13) & $0.0982(6)$ & \\
\hline $\mathrm{C} 7$ & $0.24416(10)$ & $0.6583(4)$ & $0.53037(16)$ & $0.0976(7)$ & \\
\hline $\mathrm{N} 2$ & $0.44070(10)$ & $0.3861(5)$ & $0.49826(17)$ & $0.1221(9)$ & \\
\hline $\mathrm{C} 13$ & $0.26247(11)$ & $0.5592(3)$ & $0.39102(16)$ & $0.0990(7)$ & \\
\hline H13 & 0.297280 & 0.557512 & 0.402389 & $0.119^{*}$ & \\
\hline N3 & $0.39562(11)$ & $0.3512(5)$ & 0.7007 (2) & $0.1341(10)$ & \\
\hline C8 & $0.22811(9)$ & $0.6113(3)$ & $0.44877(16)$ & $0.0912(7)$ & \\
\hline $\mathrm{O} 1$ & $0.36955(13)$ & $0.4498(6)$ & $0.73879(15)$ & $0.1791(15)$ & \\
\hline C6 & $0.31291(13)$ & $0.6898(5)$ & $0.61932(18)$ & $0.1201(10)$ & \\
\hline H6A & 0.304906 & 0.581714 & 0.651065 & $0.144^{*}$ & \\
\hline H6B & 0.296435 & 0.795389 & 0.643718 & $0.144^{*}$ & \\
\hline $\mathrm{C} 5$ & $0.36982(14)$ & $0.7184(5)$ & $0.6162(2)$ & $0.1214(10)$ & \\
\hline $\mathrm{H} 5 \mathrm{~A}$ & 0.377022 & 0.826914 & 0.584294 & $0.146^{*}$ & \\
\hline H5B & 0.382282 & 0.741023 & 0.670087 & $0.146^{*}$ & \\
\hline $\mathrm{C} 1$ & $0.41726(10)$ & $0.5501(5)$ & $0.50779(16)$ & $0.1076(9)$ & \\
\hline $\mathrm{C} 2$ & $0.43619(11)$ & $0.2967(5)$ & $0.5682(2)$ & $0.1165(9)$ & \\
\hline $\mathrm{H} 2$ & 0.449468 & 0.180669 & 0.579041 & $0.140 *$ & \\
\hline C12 & $0.24521(15)$ & $0.5095(5)$ & 0.31615 (19) & $0.1220(10)$ & \\
\hline H12 & 0.268778 & 0.474998 & 0.277171 & $0.146^{*}$ & \\
\hline $\mathrm{C} 9$ & $0.17676(12)$ & $0.6158(5)$ & $0.4293(2)$ & $0.1239(10)$ & \\
\hline H9 & 0.152766 & 0.653022 & 0.467173 & $0.149^{*}$ & \\
\hline $\mathrm{O} 2$ & $0.41029(16)$ & $0.2059(6)$ & $0.7247(3)$ & $0.2129(19)$ & \\
\hline $\mathrm{C} 3$ & $0.40976(10)$ & $0.3995(4)$ & $0.61981(18)$ & $0.1060(8)$ & \\
\hline $\mathrm{C} 10$ & $0.16136(17)$ & $0.5646(7)$ & $0.3534(3)$ & $0.1490(15)$ & \\
\hline H10 & 0.126798 & 0.568399 & 0.340365 & $0.179^{*}$ & \\
\hline C11 & $0.1952(2)$ & $0.5094(6)$ & $0.2979(2)$ & 0.1391 (13) & \\
\hline H11 & 0.184141 & 0.471593 & 0.247515 & $0.167^{*}$ & \\
\hline $\mathrm{C} 4$ & $0.41453(17)$ & $0.6957(7)$ & $0.4471(3)$ & $0.1555(16)$ & \\
\hline $\mathrm{H} 4 \mathrm{~A}$ & 0.380368 & 0.743804 & 0.445026 & $0.233^{*}$ & \\
\hline H4B & 0.423554 & 0.646143 & 0.395651 & $0.233^{*}$ & \\
\hline $\mathrm{H} 4 \mathrm{C}$ & 0.437869 & 0.792578 & 0.460914 & $0.233^{*}$ & \\
\hline $\mathrm{O} 1^{\prime}$ & 0.4899 (3) & $0.1937(13)$ & $0.3802(4)$ & $0.138(3)$ & 0.5 \\
\hline $\mathrm{H} 1^{\prime}$ & 0.462376 & 0.171352 & 0.401729 & $0.207^{*}$ & 0.5 \\
\hline $\mathrm{C}^{\prime}$ & $0.4823(3)$ & $0.2427(12)$ & $0.3078(5)$ & $0.106(2)$ & 0.5 \\
\hline $\mathrm{O} 2^{\prime}$ & $0.4576(4)$ & $0.3627(13)$ & $0.2891(5)$ & $0.213(4)$ & 0.5 \\
\hline
\end{tabular}




\begin{tabular}{|c|c|c|c|c|c|}
\hline $\mathrm{C} 2^{\prime}$ & 0.500000 & $0.1017(7)$ & 0.250000 & $0.1213(13)$ & \\
\hline $\mathrm{O} 2 "$ & $0.5116(4)$ & $0.1127(9)$ & $0.3942(4)$ & $0.152(2)$ & 0.5 \\
\hline C1" & $0.4925(3)$ & $0.1738(11)$ & $0.3373(6)$ & $0.105(2)$ & 0.5 \\
\hline O1" & $0.4611(3)$ & $0.3098(12)$ & $0.3421(5)$ & $0.185(3)$ & 0.5 \\
\hline H1" & 0.450297 & 0.317907 & 0.388154 & $0.277^{*}$ & 0.5 \\
\hline
\end{tabular}

Atomic displacement parameters $\left(\AA^{2}\right)$

\begin{tabular}{lllllll}
\hline & $U^{11}$ & $U^{22}$ & $U^{33}$ & $U^{12}$ & $U^{13}$ & $U^{23}$ \\
\hline O3 & $0.0978(12)$ & $0.1145(12)$ & $0.0883(11)$ & $0.0141(8)$ & $0.0050(8)$ & $0.0030(8)$ \\
O4 & $0.1174(15)$ & $0.190(2)$ & $0.1145(16)$ & $0.0319(14)$ & $0.0284(12)$ & $-0.0079(15)$ \\
N1 & $0.0877(12)$ & $0.1129(14)$ & $0.0940(13)$ & $-0.0032(10)$ & $-0.0056(9)$ & $-0.0007(10)$ \\
C7 & $0.0945(15)$ & $0.0968(14)$ & $0.1015(16)$ & $0.0193(11)$ & $0.0169(12)$ & $0.0104(12)$ \\
N2 & $0.0934(14)$ & $0.160(2)$ & $0.1128(17)$ & $-0.0092(14)$ & $0.0132(12)$ & $-0.0241(16)$ \\
C13 & $0.1102(17)$ & $0.0899(14)$ & $0.0968(16)$ & $-0.0003(12)$ & $0.0094(12)$ & $0.0096(12)$ \\
N3 & $0.1095(18)$ & $0.169(3)$ & $0.124(2)$ & $0.0232(17)$ & $0.0120(15)$ & $0.0390(19)$ \\
C8 & $0.0917(14)$ & $0.0806(12)$ & $0.1014(15)$ & $0.0051(10)$ & $0.0060(11)$ & $0.0209(11)$ \\
O1 & $0.159(2)$ & $0.275(4)$ & $0.1037(16)$ & $0.063(3)$ & $0.0161(15)$ & $0.026(2)$ \\
C6 & $0.120(2)$ & $0.150(2)$ & $0.0906(16)$ & $0.0267(18)$ & $-0.0044(14)$ & $-0.0155(16)$ \\
C5 & $0.130(2)$ & $0.118(2)$ & $0.116(2)$ & $0.0064(17)$ & $-0.0114(17)$ & $-0.0212(16)$ \\
C1 & $0.0871(14)$ & $0.141(2)$ & $0.0945(16)$ & $-0.0176(14)$ & $-0.0003(11)$ & $0.0035(15)$ \\
C2 & $0.0947(17)$ & $0.124(2)$ & $0.131(2)$ & $-0.0053(14)$ & $0.0115(16)$ & $-0.0025(17)$ \\
C12 & $0.151(3)$ & $0.1107(19)$ & $0.1046(19)$ & $-0.0162(19)$ & $0.0084(19)$ & $0.0106(16)$ \\
C9 & $0.0969(18)$ & $0.140(2)$ & $0.135(3)$ & $0.0034(16)$ & $0.0031(17)$ & $0.025(2)$ \\
O2 & $0.197(3)$ & $0.230(4)$ & $0.212(4)$ & $0.064(3)$ & $0.060(3)$ & $0.109(3)$ \\
C3 & $0.0827(14)$ & $0.1249(19)$ & $0.1105(18)$ & $-0.0017(13)$ & $0.0031(12)$ & $0.0086(15)$ \\
C10 & $0.126(3)$ & $0.165(3)$ & $0.157(4)$ & $-0.015(2)$ & $-0.040(3)$ & $0.038(3)$ \\
C11 & $0.171(3)$ & $0.129(2)$ & $0.117(2)$ & $-0.039(3)$ & $-0.026(2)$ & $0.034(2)$ \\
C4 & $0.131(3)$ & $0.207(4)$ & $0.128(3)$ & $-0.021(3)$ & $0.000(2)$ & $0.048(3)$ \\
O1' & $0.141(5)$ & $0.174(6)$ & $0.099(4)$ & $0.044(4)$ & $0.001(3)$ & $0.000(4)$ \\
C1' & $0.089(4)$ & $0.126(6)$ & $0.104(4)$ & $0.017(4)$ & $0.004(3)$ & $0.013(4)$ \\
O2' & $0.302(10)$ & $0.225(7)$ & $0.112(4)$ & $0.145(7)$ & $0.025(5)$ & $0.010(5)$ \\
C2' & $0.131(3)$ & $0.133(3)$ & $0.099(3)$ & 0.000 & $-0.001(2)$ & 0.000 \\
O2" & $0.210(7)$ & $0.134(4)$ & $0.111(4)$ & $0.034(4)$ & $-0.017(4)$ & $-0.010(3)$ \\
C1" & $0.079(4)$ & $0.099(5)$ & $0.135(8)$ & $0.003(3)$ & $0.015(5)$ & $0.010(5)$ \\
O1" & $0.203(7)$ & $0.218(7)$ & $0.134(5)$ & $0.106(6)$ & $0.029(5)$ & $0.018(5)$ \\
& & & & & &
\end{tabular}

Geometric parameters $\left(\AA,{ }^{\circ}\right)$

\begin{tabular}{llll}
\hline $\mathrm{O} 3-\mathrm{C} 7$ & $1.344(3)$ & $\mathrm{C} 8-\mathrm{C} 9$ & $1.382(4)$ \\
$\mathrm{O} 3-\mathrm{C} 6$ & $1.428(4)$ & $\mathrm{C} 6-\mathrm{C} 5$ & $1.504(5)$ \\
$\mathrm{O} 4-\mathrm{C} 7$ & $1.210(3)$ & $\mathrm{C} 1-\mathrm{C} 4$ & $1.466(5)$ \\
$\mathrm{N} 1-\mathrm{C} 5$ & $1.469(4)$ & $\mathrm{C} 2-\mathrm{C} 3$ & $1.333(4)$ \\
$\mathrm{N} 1-\mathrm{C} 1$ & $1.343(4)$ & $\mathrm{C} 12-\mathrm{C} 11$ & $1.344(5)$ \\
$\mathrm{N} 1-\mathrm{C} 3$ & $1.371(4)$ & $\mathrm{C} 9-\mathrm{C} 10$ & $1.380(6)$ \\
$\mathrm{C} 7-\mathrm{C} 8$ & $1.464(4)$ & $\mathrm{C} 10-\mathrm{C} 11$ & $1.341(6)$ \\
$\mathrm{N} 2-\mathrm{C} 1$ & $1.350(4)$ & $\mathrm{O} 1^{\prime}-\mathrm{C} 1^{\prime}$ & $1.274(9)$ \\
$\mathrm{N} 2-\mathrm{C} 2$ & $1.340(4)$ & $\mathrm{C} 1^{\prime}-\mathrm{O} 2^{\prime}$ & $1.129(9)$
\end{tabular}




\begin{tabular}{|c|c|c|c|}
\hline $\mathrm{C} 13-\mathrm{C} 8$ & $1.370(4)$ & $\mathrm{C} 1^{\prime}-\mathrm{C} 2^{\prime}$ & $1.482(9)$ \\
\hline $\mathrm{C} 13-\mathrm{C} 12$ & $1.376(4)$ & $\mathrm{C} 2^{\prime}-\mathrm{C} 1 "$ & $1.559(10)$ \\
\hline $\mathrm{N} 3-\mathrm{O} 1$ & $1.176(4)$ & $\mathrm{C} 2^{\prime}-\mathrm{C} 1^{\prime \prime i}$ & $1.559(10)$ \\
\hline $\mathrm{N} 3-\mathrm{O} 2$ & $1.193(4)$ & $\mathrm{O} 2 "-\mathrm{C} 1 "$ & $1.161(11)$ \\
\hline $\mathrm{N} 3-\mathrm{C} 3$ & $1.441(4)$ & $\mathrm{C} 1 "-\mathrm{O} 1 "$ & $1.288(9)$ \\
\hline $\mathrm{C} 7-\mathrm{O} 3-\mathrm{C} 6$ & $115.3(2)$ & $\mathrm{N} 2-\mathrm{C} 1-\mathrm{C} 4$ & $125.4(3)$ \\
\hline $\mathrm{C} 1-\mathrm{N} 1-\mathrm{C} 5$ & $126.4(3)$ & $\mathrm{C} 3-\mathrm{C} 2-\mathrm{N} 2$ & $109.6(3)$ \\
\hline $\mathrm{C} 1-\mathrm{N} 1-\mathrm{C} 3$ & $106.4(2)$ & $\mathrm{C} 11-\mathrm{C} 12-\mathrm{C} 13$ & $121.7(3)$ \\
\hline $\mathrm{C} 3-\mathrm{N} 1-\mathrm{C} 5$ & $127.1(3)$ & $\mathrm{C} 10-\mathrm{C} 9-\mathrm{C} 8$ & $119.5(3)$ \\
\hline $\mathrm{O} 3-\mathrm{C} 7-\mathrm{C} 8$ & $113.2(2)$ & $\mathrm{N} 1-\mathrm{C} 3-\mathrm{N} 3$ & $125.4(3)$ \\
\hline $\mathrm{O} 4-\mathrm{C} 7-\mathrm{O} 3$ & $121.9(3)$ & $\mathrm{C} 2-\mathrm{C} 3-\mathrm{N} 1$ & $107.7(3)$ \\
\hline $\mathrm{O} 4-\mathrm{C} 7-\mathrm{C} 8$ & $124.9(3)$ & $\mathrm{C} 2-\mathrm{C} 3-\mathrm{N} 3$ & $126.9(3)$ \\
\hline $\mathrm{C} 2-\mathrm{N} 2-\mathrm{C} 1$ & $106.6(3)$ & $\mathrm{C} 11-\mathrm{C} 10-\mathrm{C} 9$ & $121.4(4)$ \\
\hline $\mathrm{C} 8-\mathrm{C} 13-\mathrm{C} 12$ & $119.7(3)$ & $\mathrm{C} 10-\mathrm{C} 11-\mathrm{C} 12$ & $119.1(4)$ \\
\hline $\mathrm{O} 1-\mathrm{N} 3-\mathrm{O} 2$ & $122.9(4)$ & $\mathrm{O} 1^{\prime}-\mathrm{C} 1^{\prime}-\mathrm{C} 2^{\prime}$ & $112.0(7)$ \\
\hline $\mathrm{O} 1-\mathrm{N} 3-\mathrm{C} 3$ & $120.4(3)$ & $\mathrm{O} 2^{\prime}-\mathrm{C} 1^{\prime}-\mathrm{O} 1^{\prime}$ & $124.5(8)$ \\
\hline $\mathrm{O} 2-\mathrm{N} 3-\mathrm{C} 3$ & $116.6(4)$ & $\mathrm{O} 2^{\prime}-\mathrm{C} 1^{\prime}-\mathrm{C} 2^{\prime}$ & $122.3(7)$ \\
\hline $\mathrm{C} 13-\mathrm{C} 8-\mathrm{C} 7$ & $122.0(2)$ & $\mathrm{C}^{\prime \prime}-\mathrm{C} 2^{\prime}-\mathrm{C} 1^{\prime}$ & $92.4(7)$ \\
\hline $\mathrm{C} 13-\mathrm{C} 8-\mathrm{C} 9$ & $118.6(3)$ & $\mathrm{C} 1^{\prime \mathrm{i}}-\mathrm{C} 2^{\prime}-\mathrm{C} 1^{\prime \prime} \mathrm{i}$ & $28.4(3)$ \\
\hline $\mathrm{C} 9-\mathrm{C} 8-\mathrm{C} 7$ & $119.4(3)$ & $\mathrm{C} 1^{\prime}-\mathrm{C} 2^{\prime}-\mathrm{C} 1^{\prime \prime}$ & $114.4(6)$ \\
\hline $\mathrm{O} 3-\mathrm{C} 6-\mathrm{C} 5$ & $107.8(3)$ & $\mathrm{C} 1 " \mathrm{i}-\mathrm{C} 2{ }^{\prime}-\mathrm{C} 1 "$ & $140.7(7)$ \\
\hline $\mathrm{N} 1-\mathrm{C} 5-\mathrm{C} 6$ & $113.4(3)$ & $\mathrm{O} 2 "-\mathrm{C} 1 "-\mathrm{C} 2{ }^{\prime}$ & $125.5(7)$ \\
\hline $\mathrm{N} 1-\mathrm{C} 1-\mathrm{N} 2$ & $109.6(3)$ & $\mathrm{O} 2 "-\mathrm{C} 1 "-\mathrm{O} 1 "$ & $121.1(10)$ \\
\hline $\mathrm{N} 1-\mathrm{C} 1-\mathrm{C} 4$ & $125.0(3)$ & $\mathrm{O} 1 "-\mathrm{C} 1 "-\mathrm{C} 2^{\prime}$ & $113.4(8)$ \\
\hline
\end{tabular}

Symmetry code: (i) $-x+1, y,-z+1 / 2$.

1-[2-(Benzoyloxy)ethyl]-2-methyl-5-nitro-1 H-imidazol-3-ium 2,6-dihydroxybenzoate (bzmd26dba)

\section{Crystal data}

$\mathrm{C}_{13} \mathrm{H}_{14} \mathrm{~N}_{3} \mathrm{O}_{4}^{+} \cdot \mathrm{C}_{7} \mathrm{H}_{5} \mathrm{O}_{4}^{-}$

$M_{r}=429.38$

Monoclinic, $P 2_{1} / n$

$a=8.2443(4) \AA$

$b=15.9009(7) \AA$

$c=15.4526(8) \AA$

$\beta=102.454(2)^{\circ}$

$V=1978.04(17) \AA^{3}$

$Z=4$

\section{Data collection}

Bruker D8 VENTURE Kappa Duo PHOTON II CPAD diffractometer

$\varphi$ and $\omega$ scans

Absorption correction: multi-scan

(SADABS; Bruker, 2016)

$T_{\min }=0.490, T_{\max }=0.746$

27674 measured reflections
$F(000)=896$

$D_{\mathrm{x}}=1.442 \mathrm{Mg} \mathrm{m}^{-3}$

Mo $K \alpha$ radiation, $\lambda=0.71073 \AA$

Cell parameters from 9886 reflections

$\theta=2.5-27.5^{\circ}$

$\mu=0.11 \mathrm{~mm}^{-1}$

$T=301 \mathrm{~K}$

Prism, clear light yellow

$0.83 \times 0.67 \times 0.27 \mathrm{~mm}$

4348 independent reflections

3408 reflections with $I>2 \sigma(I)$

$R_{\text {int }}=0.076$

$\theta_{\text {max }}=27.5^{\circ}, \theta_{\min }=2.8^{\circ}$

$h=-10 \rightarrow 10$

$k=-20 \rightarrow 20$

$l=-20 \rightarrow 19$ 


\section{Refinement}

Refinement on $F^{2}$

Least-squares matrix: full

$R\left[F^{2}>2 \sigma\left(F^{2}\right)\right]=0.069$

$w R\left(F^{2}\right)=0.185$

$S=1.10$

4348 reflections

356 parameters

0 restraints
Hydrogen site location: difference Fourier map

All $\mathrm{H}$-atom parameters refined

$w=1 /\left[\sigma^{2}\left(F_{\mathrm{o}}^{2}\right)+(0.0913 P)^{2}+0.5735 P\right]$

where $P=\left(F_{\mathrm{o}}{ }^{2}+2 F_{\mathrm{c}}{ }^{2}\right) / 3$

$(\Delta / \sigma)_{\max }<0.001$

$\Delta \rho_{\max }=0.37 \mathrm{e}^{-3}$

$\Delta \rho_{\min }=-0.27$ e $\AA^{-3}$

Special details

Geometry. All esds (except the esd in the dihedral angle between two 1.s. planes) are estimated using the full covariance matrix. The cell esds are taken into account individually in the estimation of esds in distances, angles and torsion angles; correlations between esds in cell parameters are only used when they are defined by crystal symmetry. An approximate (isotropic) treatment of cell esds is used for estimating esds involving l.s. planes.

Refinement. Single-crystal X-ray diffraction data collection ( scans and $\omega$ scans with $\kappa$ and $\theta$ offsets) were performed at room temperature on a Bruker AXS D8 VENTURE equipped with a Kappa goniometer, PHOTON II CPAD detector, a Mo $K \alpha$ INCOATEC I $\mu$ S 3.0 microfocus source $(\lambda=0.71073 \AA)$. The crystal centring, unit-cell determination, refinement of the unit-cell parameters and data collection was controlled through the program APEX3 (Bruker, 2012). The frame integration was performed using SAINT (Bruker, 2016) and the intensities were scaled and absorption corrected using SADABS (Bruker, 2001). Using OLEX2 (Dolomanov et al., 2009), the structure was solved by intrinsic phasing using SHELXT (Sheldrick, 2015) and refined by full-matrix least-squares calculation based on $F^{2}$ for all reflection using SHELXL (Sheldrick, 2007). All non-H atoms were refined anisotropically.

Fractional atomic coordinates and isotropic or equivalent isotropic displacement parameters $\left(\hat{A}^{2}\right)$

\begin{tabular}{lllll}
\hline & $x$ & $y$ & $z$ & $U_{\text {iso }} * / U_{\text {eq }}$ \\
\hline O3 & $0.30908(16)$ & $0.45737(9)$ & $0.10470(8)$ & $0.0471(3)$ \\
O1' & $0.69550(19)$ & $0.38992(10)$ & $0.49968(10)$ & $0.0592(4)$ \\
N1 & $0.25612(18)$ & $0.49805(10)$ & $0.28713(10)$ & $0.0407(4)$ \\
O2 & $-0.08963(19)$ & $0.47761(14)$ & $0.24372(12)$ & $0.0777(6)$ \\
O4' & $1.0124(2)$ & $0.38277(12)$ & $0.56331(14)$ & $0.0725(5)$ \\
O2 $^{\prime}$ & $0.5209(2)$ & $0.32169(12)$ & $0.56404(14)$ & $0.0728(5)$ \\
N2 & $0.4226(2)$ & $0.44392(10)$ & $0.40318(11)$ & $0.0478(4)$ \\
N3 & $-0.0133(2)$ & $0.44450(12)$ & $0.31200(12)$ & $0.0545(5)$ \\
O1 & $-0.0783(2)$ & $0.40294(14)$ & $0.36109(14)$ & $0.0832(6)$ \\
O4 & $0.1499(2)$ & $0.39405(13)$ & $-0.01207(12)$ & $0.0778(6)$ \\
O3' & $0.6249(3)$ & $0.22744(13)$ & $0.69473(15)$ & $0.0855(6)$ \\
C1' & $0.8096(3)$ & $0.30675(11)$ & $0.62457(12)$ & $0.0455(4)$ \\
C1 & $0.4158(2)$ & $0.48952(12)$ & $0.33097(13)$ & $0.0441(4)$ \\
C7' & $0.6658(3)$ & $0.33991(13)$ & $0.55944(13)$ & $0.0485(5)$ \\
C8 & $0.4427(2)$ & $0.37835(12)$ & $0.01068(13)$ & $0.0448(4)$ \\
C6 & $0.1575(2)$ & $0.48994(15)$ & $0.12428(14)$ & $0.0494(5)$ \\
C3 & $0.1624(2)$ & $0.45427(12)$ & $0.33634(12)$ & $0.0435(4)$ \\
C2 & $0.2662(3)$ & $0.42133(13)$ & $0.40789(13)$ & $0.0487(5)$ \\
C5 & $0.2007(3)$ & $0.54528(13)$ & $0.20416(14)$ & $0.0479(5)$ \\
C2 & $0.9742(3)$ & $0.32956(13)$ & $0.62356(14)$ & $0.0531(5)$ \\
C7 & $0.2864(2)$ & $0.40967(13)$ & $0.03172(13)$ & $0.0484(5)$ \\
C13 & $0.4327(3)$ & $0.33220(15)$ & $-0.06576(17)$ & $0.0618(6)$ \\
C9 & $0.5971(3)$ & $0.39351(15)$ & $0.06467(16)$ & $0.0563(5)$ \\
& & & &
\end{tabular}




$\begin{array}{lllll}\text { C3' } & 1.1037(4) & 0.29727(17) & 0.6877(2) & 0.0774(8) \\ \text { C4 } & 0.5618(3) & 0.5256(2) & 0.3029(2) & 0.0649(6) \\ \text { C10 } & 0.7374(3) & 0.36102(18) & 0.04179(19) & 0.0694(7) \\ \text { C12 } & 0.5745(4) & 0.30044(16) & -0.08794(19) & 0.0693(7) \\ \text { C6 }^{\prime} & 0.7806(4) & 0.25214(13) & 0.69090(15) & 0.0616(6) \\ \text { C11 } & 0.7265(3) & 0.31502(16) & -0.03340(19) & 0.0669(6) \\ \text { C4' } & 1.0704(5) & 0.24431(17) & 0.7517(2) & 0.0892(11) \\ \text { C5' } & 0.9118(5) & 0.22182(16) & 0.75427(19) & 0.0829(9) \\ \text { H5A } & 0.292(3) & 0.5844(13) & 0.2002(14) & 0.048(5)^{*} \\ \text { H6A } & 0.086(3) & 0.4400(15) & 0.1351(16) & 0.058(6)^{*} \\ \text { H2 } & 0.238(3) & 0.3877(14) & 0.4495(17) & 0.058(7)^{*} \\ \text { H5B } & 0.109(3) & 0.5774(15) & 0.2123(15) & 0.057(6)^{*} \\ \text { H6B } & 0.099(3) & 0.5229(15) & 0.0737(18) & 0.065(7)^{*} \\ \text { H12 } & 0.564(4) & 0.2705(17) & -0.142(2) & 0.081(8)^{*} \\ \text { H9 } & 0.599(3) & 0.4257(16) & 0.1141(18) & 0.063(7)^{*} \\ \text { H3' } & 1.210(4) & 0.3103(19) & 0.676(2) & 0.088(9)^{*} \\ \text { H11 } & 0.823(4) & 0.2909(16) & -0.0509(18) & 0.074(8)^{*} \\ \text { H4'A } & 1.152(4) & 0.2196(19) & 0.798(2) & 0.095(10)^{*} \\ \text { H4' } & 0.898(5) & 0.399(2) & 0.523(2) & 0.116(12)^{*} \\ \text { H10 } & 0.843(4) & 0.370(2) & 0.082(2) & 0.100(10)^{*} \\ \text { H13 } & 0.330(4) & 0.3231(18) & -0.103(2) & 0.086(9)^{*} \\ \text { H5' } & 0.879(4) & 0.187(2) & 0.801(2) & 0.102(10)^{*} \\ \text { H2' } & 0.562(5) & 0.270(3) & 0.638(3) & 0.133(14)^{*} \\ \text { H1' } & 0.548(6) & 0.415(2) & 0.454(3) & 0.132(13)^{*} \\ \text { H4A } & 0.575(5) & 0.504(2) & 0.250(3) & 0.117(13)^{*} \\ \text { H4B } & 0.554(6) & 0.585(3) & 0.303(3) & 0.133(15)^{*} \\ \text { H4C } & 0.653(6) & 0.515(3) & 0.347(3) & 0.138(15)^{*} \\ & & & & \end{array}$

Atomic displacement parameters $\left(\AA^{2}\right)$

\begin{tabular}{lllllll}
\hline & $U^{11}$ & $U^{22}$ & $U^{33}$ & $U^{12}$ & $U^{13}$ & $U^{23}$ \\
\hline O3 & $0.0316(7)$ & $0.0630(8)$ & $0.0445(7)$ & $0.0046(6)$ & $0.0037(5)$ & $-0.0008(6)$ \\
O1' & $0.0422(8)$ & $0.0778(10)$ & $0.0546(8)$ & $0.0077(7)$ & $0.0037(6)$ & $0.0114(7)$ \\
N1 & $0.0297(8)$ & $0.0472(8)$ & $0.0446(8)$ & $0.0045(6)$ & $0.0068(6)$ & $-0.0010(6)$ \\
O2 & $0.0341(8)$ & $0.1341(16)$ & $0.0631(10)$ & $0.0079(9)$ & $0.0061(7)$ & $0.0170(10)$ \\
O4 $^{\prime}$ & $0.0427(9)$ & $0.0877(12)$ & $0.0845(12)$ & $-0.0045(8)$ & $0.0080(8)$ & $0.0172(9)$ \\
O2 $^{\prime}$ & $0.0443(9)$ & $0.0815(11)$ & $0.0917(13)$ & $-0.0026(8)$ & $0.0130(8)$ & $0.0032(10)$ \\
N2 & $0.0387(9)$ & $0.0542(9)$ & $0.0468(9)$ & $0.0072(7)$ & $0.0006(7)$ & $-0.0072(7)$ \\
N3 & $0.0333(9)$ & $0.0752(12)$ & $0.0560(10)$ & $0.0015(8)$ & $0.0114(7)$ & $0.0005(8)$ \\
O1 & $0.0479(10)$ & $0.1147(15)$ & $0.0903(13)$ & $-0.0120(10)$ & $0.0222(9)$ & $0.0255(11)$ \\
O4 & $0.0375(9)$ & $0.1101(14)$ & $0.0769(11)$ & $0.0044(8)$ & $-0.0073(8)$ & $-0.0305(10)$ \\
O3' & $0.0927(16)$ & $0.0810(12)$ & $0.0888(14)$ & $-0.0202(11)$ & $0.0326(12)$ & $0.0109(11)$ \\
C1 $1^{\prime}$ & $0.0491(11)$ & $0.0408(9)$ & $0.0442(9)$ & $0.0021(8)$ & $0.0048(8)$ & $-0.0074(7)$ \\
C1 & $0.0318(9)$ & $0.0482(10)$ & $0.0511(10)$ & $0.0044(7)$ & $0.0061(7)$ & $-0.0099(8)$ \\
C7' & $0.0411(11)$ & $0.0518(10)$ & $0.0517(11)$ & $0.0025(8)$ & $0.0078(8)$ & $-0.0100(8)$ \\
C8 & $0.0391(10)$ & $0.0469(9)$ & $0.0474(10)$ & $0.0003(8)$ & $0.0071(8)$ & $0.0036(8)$ \\
C6 & $0.0315(10)$ & $0.0673(13)$ & $0.0481(10)$ & $0.0081(9)$ & $0.0057(8)$ & $0.0033(9)$ \\
C3 & $0.0339(10)$ & $0.0502(10)$ & $0.0461(9)$ & $0.0032(7)$ & $0.0076(7)$ & $-0.0035(8)$
\end{tabular}


supporting information

\begin{tabular}{lllllll} 
& & & & \\
C2 & $0.0459(11)$ & $0.0546(11)$ & $0.0445(10)$ & $0.0032(9)$ & $0.0075(8)$ & $-0.0003(8)$ \\
C5 & $0.0411(11)$ & $0.0499(10)$ & $0.0532(11)$ & $0.0095(9)$ & $0.0110(8)$ & $0.0076(8)$ \\
C2' & $0.0481(12)$ & $0.0484(10)$ & $0.0569(11)$ & $0.0023(8)$ & $-0.0017(9)$ & $-0.0042(9)$ \\
C7 & $0.0371(10)$ & $0.0581(11)$ & $0.0472(10)$ & $0.0012(8)$ & $0.0026(8)$ & $0.0017(8)$ \\
C13 & $0.0543(14)$ & $0.0662(14)$ & $0.0628(13)$ & $-0.0018(11)$ & $0.0084(11)$ & $-0.0111(11)$ \\
C9 & $0.0408(11)$ & $0.0693(13)$ & $0.0559(12)$ & $0.0008(9)$ & $0.0040(9)$ & $-0.0047(10)$ \\
C3' & $0.0580(16)$ & $0.0624(14)$ & $0.0941(19)$ & $0.0003(12)$ & $-0.0227(14)$ & $-0.0016(13)$ \\
C4 & $0.0343(12)$ & $0.0828(18)$ & $0.0783(17)$ & $-0.0058(11)$ & $0.0140(11)$ & $-0.0068(14)$ \\
C10 & $0.0387(13)$ & $0.0857(17)$ & $0.0824(17)$ & $0.0056(11)$ & $0.0097(11)$ & $-0.0019(14)$ \\
C12 & $0.0786(18)$ & $0.0628(14)$ & $0.0718(15)$ & $0.0038(12)$ & $0.0281(13)$ & $-0.0111(12)$ \\
C6' & $0.0806(17)$ & $0.0450(10)$ & $0.0571(12)$ & $-0.0043(10)$ & $0.0104(11)$ & $-0.0039(9)$ \\
C11 & $0.0559(15)$ & $0.0649(14)$ & $0.0858(17)$ & $0.0122(11)$ & $0.0286(13)$ & $0.0062(12)$ \\
C4' & $0.106(3)$ & $0.0537(14)$ & $0.0816(18)$ & $0.0071(14)$ & $-0.0370(17)$ & $0.0058(13)$ \\
C5' & $0.117(3)$ & $0.0514(13)$ & $0.0667(15)$ & $-0.0072(14)$ & $-0.0101(16)$ & $0.0113(12)$ \\
& & & & & & \\
\hline
\end{tabular}

Geometric parameters $\left(A,{ }^{\circ}\right)$

\begin{tabular}{|c|c|c|c|}
\hline $\mathrm{O} 3-\mathrm{C} 6$ & $1.444(2)$ & $\mathrm{C} 6-\mathrm{C} 5$ & $1.495(3)$ \\
\hline $\mathrm{O} 3-\mathrm{C} 7$ & $1.338(2)$ & $\mathrm{C} 6-\mathrm{H} 6 \mathrm{~A}$ & $1.02(2)$ \\
\hline $\mathrm{O} 1^{\prime}-\mathrm{C}^{\prime}$ & $1.282(3)$ & C6-H6B & $0.98(3)$ \\
\hline $\mathrm{O} 1^{\prime}-\mathrm{H} 1^{\prime}$ & $1.32(5)$ & $\mathrm{C} 3-\mathrm{C} 2$ & $1.350(3)$ \\
\hline $\mathrm{N} 1-\mathrm{C} 1$ & $1.351(2)$ & $\mathrm{C} 2-\mathrm{H} 2$ & $0.90(2)$ \\
\hline $\mathrm{N} 1-\mathrm{C} 3$ & $1.383(2)$ & $\mathrm{C} 5-\mathrm{H} 5 \mathrm{~A}$ & $0.99(2)$ \\
\hline $\mathrm{N} 1-\mathrm{C} 5$ & $1.471(2)$ & C5-H5B & $0.95(2)$ \\
\hline $\mathrm{O} 2-\mathrm{N} 3$ & $1.225(2)$ & $\mathrm{C} 2^{\prime}-\mathrm{C} 3^{\prime}$ & $1.389(3)$ \\
\hline $\mathrm{O} 4^{\prime}-\mathrm{C} 2^{\prime}$ & $1.345(3)$ & $\mathrm{C} 13-\mathrm{C} 12$ & $1.383(4)$ \\
\hline $\mathrm{O} 4^{\prime}-\mathrm{H} 4^{\prime}$ & $1.05(4)$ & $\mathrm{C} 13-\mathrm{H} 13$ & $0.93(3)$ \\
\hline $\mathrm{O} 2^{\prime}-\mathrm{C}^{\prime}$ & $1.246(3)$ & $\mathrm{C} 9-\mathrm{C} 10$ & $1.381(3)$ \\
\hline $\mathrm{O} 2^{\prime}-\mathrm{H} 2^{\prime}$ & $1.38(5)$ & C9- $\mathrm{H} 9$ & $0.92(3)$ \\
\hline $\mathrm{N} 2-\mathrm{C} 1$ & $1.322(3)$ & $\mathrm{C} 3^{\prime}-\mathrm{C} 4^{\prime}$ & $1.371(5)$ \\
\hline $\mathrm{N} 2-\mathrm{C} 2$ & $1.355(3)$ & $\mathrm{C} 3^{\prime}-\mathrm{H} 3^{\prime}$ & $0.96(3)$ \\
\hline $\mathrm{N} 2-\mathrm{H} 1^{\prime}$ & $1.25(5)$ & $\mathrm{C} 4-\mathrm{H} 4 \mathrm{~A}$ & $0.92(4)$ \\
\hline $\mathrm{N} 3-\mathrm{O} 1$ & $1.214(2)$ & $\mathrm{C} 4-\mathrm{H} 4 \mathrm{~B}$ & $0.95(4)$ \\
\hline $\mathrm{N} 3-\mathrm{C} 3$ & $1.424(3)$ & $\mathrm{C} 4-\mathrm{H} 4 \mathrm{C}$ & $0.92(5)$ \\
\hline $\mathrm{O} 4-\mathrm{C} 7$ & $1.207(3)$ & $\mathrm{C} 10-\mathrm{C} 11$ & $1.359(4)$ \\
\hline $\mathrm{O} 3^{\prime}-\mathrm{C} 6^{\prime}$ & $1.356(3)$ & $\mathrm{C} 10-\mathrm{H} 10$ & $0.97(4)$ \\
\hline $\mathrm{O} 3^{\prime}-\mathrm{H} 2^{\prime}$ & $1.15(5)$ & $\mathrm{C} 12-\mathrm{C} 11$ & $1.370(4)$ \\
\hline $\mathrm{C} 1^{\prime}-\mathrm{C} 7^{\prime}$ & $1.477(3)$ & $\mathrm{C} 12-\mathrm{H} 12$ & $0.94(3)$ \\
\hline $\mathrm{C} 1^{\prime}-\mathrm{C} 2^{\prime}$ & $1.408(3)$ & $\mathrm{C} 6^{\prime}-\mathrm{C} 5^{\prime}$ & $1.380(4)$ \\
\hline $\mathrm{C} 1^{\prime}-\mathrm{C} 6^{\prime}$ & $1.402(3)$ & $\mathrm{C} 11-\mathrm{H} 11$ & $0.97(3)$ \\
\hline $\mathrm{C} 1-\mathrm{C} 4$ & $1.480(3)$ & $\mathrm{C} 4^{\prime}-\mathrm{C} 5^{\prime}$ & $1.365(5)$ \\
\hline $\mathrm{C} 8-\mathrm{C} 7$ & $1.481(3)$ & $\mathrm{C} 4^{\prime}-\mathrm{H} 4^{\prime} \mathrm{A}$ & $0.96(4)$ \\
\hline $\mathrm{C} 8-\mathrm{C} 13$ & $1.378(3)$ & $\mathrm{C} 5^{\prime}-\mathrm{H} 5^{\prime}$ & $1.00(3)$ \\
\hline $\mathrm{C} 8-\mathrm{C} 9$ & $1.385(3)$ & & \\
\hline $\mathrm{C} 7-\mathrm{O} 3-\mathrm{C} 6$ & $114.21(15)$ & $\mathrm{C} 6-\mathrm{C} 5-\mathrm{H} 5 \mathrm{~B}$ & $111.8(15)$ \\
\hline $\mathrm{C} 7^{\prime}-\mathrm{O} 1^{\prime}-\mathrm{H} 1^{\prime}$ & $105.9(17)$ & $\mathrm{H} 5 \mathrm{~A}-\mathrm{C} 5-\mathrm{H} 5 \mathrm{~B}$ & $108.1(19)$ \\
\hline $\mathrm{C} 1-\mathrm{N} 1-\mathrm{C} 3$ & $105.86(15)$ & $\mathrm{O} 4^{\prime}-\mathrm{C} 2^{\prime}-\mathrm{C} 1^{\prime}$ & $122.70(19)$ \\
\hline
\end{tabular}




\begin{tabular}{|c|c|c|c|}
\hline $\mathrm{C} 1-\mathrm{N} 1-\mathrm{C} 5$ & $125.05(17)$ & $\mathrm{O} 4^{\prime}-\mathrm{C} 2^{\prime}-\mathrm{C} 3^{\prime}$ & $117.9(2)$ \\
\hline $\mathrm{C} 3-\mathrm{N} 1-\mathrm{C} 5$ & $129.09(16)$ & $\mathrm{C} 3^{\prime}-\mathrm{C} 2^{\prime}-\mathrm{C} 1^{\prime}$ & $119.4(2)$ \\
\hline $\mathrm{C} 2^{\prime}-\mathrm{O} 4^{\prime}-\mathrm{H} 4^{\prime}$ & $104.8(19)$ & $\mathrm{O} 3-\mathrm{C} 7-\mathrm{C} 8$ & $113.92(17)$ \\
\hline $\mathrm{C} 7^{\prime}-\mathrm{O} 2^{\prime}-\mathrm{H} 2^{\prime}$ & 96.7 (17) & $\mathrm{O} 4-\mathrm{C} 7-\mathrm{O} 3$ & $122.18(19)$ \\
\hline $\mathrm{C} 1-\mathrm{N} 2-\mathrm{C} 2$ & $108.84(17)$ & $\mathrm{O} 4-\mathrm{C} 7-\mathrm{C} 8$ & $123.90(19)$ \\
\hline $\mathrm{C} 1-\mathrm{N} 2-\mathrm{H} 1^{\prime}$ & $127.8(19)$ & $\mathrm{C} 8-\mathrm{C} 13-\mathrm{C} 12$ & $120.6(2)$ \\
\hline $\mathrm{C} 2-\mathrm{N} 2-\mathrm{H} 1^{\prime}$ & $122.7(18)$ & $\mathrm{C} 8-\mathrm{C} 13-\mathrm{H} 13$ & $119.3(19)$ \\
\hline $\mathrm{O} 2-\mathrm{N} 3-\mathrm{C} 3$ & $119.01(18)$ & $\mathrm{C} 12-\mathrm{C} 13-\mathrm{H} 13$ & $120.0(19)$ \\
\hline $\mathrm{O} 1-\mathrm{N} 3-\mathrm{O} 2$ & $123.94(19)$ & $\mathrm{C} 8-\mathrm{C} 9-\mathrm{H} 9$ & $116.6(17)$ \\
\hline $\mathrm{O} 1-\mathrm{N} 3-\mathrm{C} 3$ & $117.05(18)$ & $\mathrm{C} 10-\mathrm{C} 9-\mathrm{C} 8$ & $119.5(2)$ \\
\hline $\mathrm{C} 6^{\prime}-\mathrm{O} 3^{\prime}-\mathrm{H} 2^{\prime}$ & $94(2)$ & $\mathrm{C} 10-\mathrm{C} 9-\mathrm{H} 9$ & $123.9(17)$ \\
\hline $\mathrm{C} 2^{\prime}-\mathrm{C} 1^{\prime}-\mathrm{C} 7^{\prime}$ & $122.29(19)$ & $\mathrm{C} 2^{\prime}-\mathrm{C} 3^{\prime}-\mathrm{H} 3^{\prime}$ & $112(2)$ \\
\hline $\mathrm{C} 6^{\prime}-\mathrm{C} 1^{\prime}-\mathrm{C} 7^{\prime}$ & $118.7(2)$ & $\mathrm{C} 4^{\prime}-\mathrm{C} 3^{\prime}-\mathrm{C} 2^{\prime}$ & $119.9(3)$ \\
\hline $\mathrm{C} 6^{\prime}-\mathrm{C} 1^{\prime}-\mathrm{C} 2^{\prime}$ & $118.9(2)$ & $\mathrm{C} 4^{\prime}-\mathrm{C} 3^{\prime}-\mathrm{H} 3^{\prime}$ & $127.4(19)$ \\
\hline $\mathrm{N} 1-\mathrm{C} 1-\mathrm{C} 4$ & $125.5(2)$ & $\mathrm{C} 1-\mathrm{C} 4-\mathrm{H} 4 \mathrm{~A}$ & $112(2)$ \\
\hline $\mathrm{N} 2-\mathrm{C} 1-\mathrm{N} 1$ & $109.75(17)$ & $\mathrm{C} 1-\mathrm{C} 4-\mathrm{H} 4 \mathrm{~B}$ & $109(3)$ \\
\hline $\mathrm{N} 2-\mathrm{C} 1-\mathrm{C} 4$ & $124.8(2)$ & $\mathrm{C} 1-\mathrm{C} 4-\mathrm{H} 4 \mathrm{C}$ & $107(3)$ \\
\hline $\mathrm{O} 1^{\prime}-\mathrm{C} 7^{\prime}-\mathrm{C} 1^{\prime}$ & $117.54(18)$ & $\mathrm{H} 4 \mathrm{~A}-\mathrm{C} 4-\mathrm{H} 4 \mathrm{~B}$ & $113(4)$ \\
\hline $\mathrm{O} 2^{\prime}-\mathrm{C}^{\prime}-\mathrm{O} 1^{\prime}$ & $121.3(2)$ & $\mathrm{H} 4 \mathrm{~A}-\mathrm{C} 4-\mathrm{H} 4 \mathrm{C}$ & $111(4)$ \\
\hline $\mathrm{O} 2^{\prime}-\mathrm{C} 7^{\prime}-\mathrm{C} 1^{\prime}$ & $121.1(2)$ & $\mathrm{H} 4 \mathrm{~B}-\mathrm{C} 4-\mathrm{H} 4 \mathrm{C}$ & $104(4)$ \\
\hline $\mathrm{C} 13-\mathrm{C} 8-\mathrm{C} 7$ & $118.26(19)$ & $\mathrm{C} 9-\mathrm{C} 10-\mathrm{H} 10$ & $118(2)$ \\
\hline $\mathrm{C} 13-\mathrm{C} 8-\mathrm{C} 9$ & $119.1(2)$ & $\mathrm{C} 11-\mathrm{C} 10-\mathrm{C} 9$ & $121.0(2)$ \\
\hline $\mathrm{C} 9-\mathrm{C} 8-\mathrm{C} 7$ & $122.66(19)$ & $\mathrm{C} 11-\mathrm{C} 10-\mathrm{H} 10$ & $121(2)$ \\
\hline $\mathrm{O} 3-\mathrm{C} 6-\mathrm{C} 5$ & $108.75(16)$ & $\mathrm{C} 13-\mathrm{C} 12-\mathrm{H} 12$ & 118.5 (19) \\
\hline $\mathrm{O} 3-\mathrm{C} 6-\mathrm{H} 6 \mathrm{~A}$ & $108.1(13)$ & $\mathrm{C} 11-\mathrm{C} 12-\mathrm{C} 13$ & $119.7(2)$ \\
\hline $\mathrm{O} 3-\mathrm{C} 6-\mathrm{H} 6 \mathrm{~B}$ & $108.8(15)$ & $\mathrm{C} 11-\mathrm{C} 12-\mathrm{H} 12$ & $121.8(19)$ \\
\hline $\mathrm{C} 5-\mathrm{C} 6-\mathrm{H} 6 \mathrm{~A}$ & $111.4(13)$ & $\mathrm{O} 3^{\prime}-\mathrm{C} 6^{\prime}-\mathrm{C} 1^{\prime}$ & $121.5(2)$ \\
\hline $\mathrm{C} 5-\mathrm{C} 6-\mathrm{H} 6 \mathrm{~B}$ & $109.4(14)$ & $\mathrm{O} 3^{\prime}-\mathrm{C} 6^{\prime}-\mathrm{C} 5^{\prime}$ & $118.2(3)$ \\
\hline $\mathrm{H} 6 \mathrm{~A}-\mathrm{C} 6-\mathrm{H} 6 \mathrm{~B}$ & $110(2)$ & $\mathrm{C} 5^{\prime}-\mathrm{C} 6^{\prime}-\mathrm{C} 1^{\prime}$ & $120.3(3)$ \\
\hline $\mathrm{N} 1-\mathrm{C} 3-\mathrm{N} 3$ & $124.52(17)$ & $\mathrm{C} 10-\mathrm{C} 11-\mathrm{C} 12$ & $120.0(2)$ \\
\hline $\mathrm{C} 2-\mathrm{C} 3-\mathrm{N} 1$ & $108.34(17)$ & $\mathrm{C} 10-\mathrm{C} 11-\mathrm{H} 11$ & $122.8(17)$ \\
\hline $\mathrm{C} 2-\mathrm{C} 3-\mathrm{N} 3$ & $127.11(19)$ & $\mathrm{C} 12-\mathrm{C} 11-\mathrm{H} 11$ & $117.2(17)$ \\
\hline $\mathrm{N} 2-\mathrm{C} 2-\mathrm{H} 2$ & $125.8(17)$ & $\mathrm{C} 3^{\prime}-\mathrm{C} 4^{\prime}-\mathrm{H} 4^{\prime} \mathrm{A}$ & $126(2)$ \\
\hline $\mathrm{C} 3-\mathrm{C} 2-\mathrm{N} 2$ & $107.21(19)$ & $\mathrm{C} 5^{\prime}-\mathrm{C} 4^{\prime}-\mathrm{C} 3^{\prime}$ & $121.6(3)$ \\
\hline $\mathrm{C} 3-\mathrm{C} 2-\mathrm{H} 2$ & $126.8(17)$ & $\mathrm{C}^{\prime}-\mathrm{C} 4^{\prime}-\mathrm{H} 4^{\prime} \mathrm{A}$ & $113(2)$ \\
\hline $\mathrm{N} 1-\mathrm{C} 5-\mathrm{C} 6$ & 113.14 (17) & $\mathrm{C} 6^{\prime}-\mathrm{C} 5^{\prime}-\mathrm{H} 5^{\prime}$ & $115(2)$ \\
\hline $\mathrm{N} 1-\mathrm{C} 5-\mathrm{H} 5 \mathrm{~A}$ & $106.2(13)$ & $\mathrm{C} 4^{\prime}-\mathrm{C} 5^{\prime}-\mathrm{C} 6^{\prime}$ & $119.8(3)$ \\
\hline $\mathrm{N} 1-\mathrm{C} 5-\mathrm{H} 5 \mathrm{~B}$ & $105.5(14)$ & $\mathrm{C} 4^{\prime}-\mathrm{C} 5^{\prime}-\mathrm{H} 5^{\prime}$ & $126(2)$ \\
\hline $\mathrm{C} 6-\mathrm{C} 5-\mathrm{H} 5 \mathrm{~A}$ & $111.6(13)$ & & \\
\hline
\end{tabular}

2-(2-Methyl-5-nitro-1H-imidazol-1-yl)ethyl benzoate-3,5-dihydroxybenzoic acid (3/1) (bzmd35dba)

Crystal data

$3 \mathrm{C}_{13} \mathrm{H}_{13} \mathrm{~N}_{3} \mathrm{O}_{4} \cdot \mathrm{C}_{7} \mathrm{H}_{6} \mathrm{O}_{4}$

$M_{r}=979.91$

Monoclinic, $P 2_{1} / n$

$a=7.1481$ (2) $\AA$

$b=36.7892(8) \AA$

$c=18.0584$ (4) $\AA$

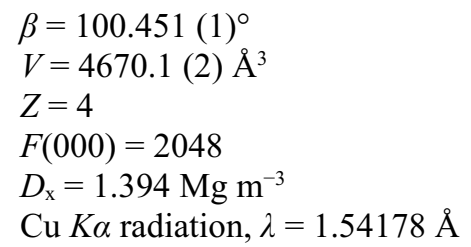


Cell parameters from 9896 reflections

$\theta=3.5-67.6^{\circ}$

$\mu=0.91 \mathrm{~mm}^{-1}$

\section{Data collection}

Bruker APEXII CCD

diffractometer

Multilayer mirrors monochromator $\varphi$ and $\omega$ scans

Absorption correction: multi-scan

(SADABS; Bruker, 2016)

$T_{\min }=0.835, T_{\max }=0.986$

56516 measured reflections

\section{Refinement}

Refinement on $F^{2}$

Least-squares matrix: full

$R\left[F^{2}>2 \sigma\left(F^{2}\right)\right]=0.052$

$w R\left(F^{2}\right)=0.176$

$S=1.01$

8547 reflections

844 parameters

1 restraint

Primary atom site location: dual

Hydrogen site location: mixed
$T=300 \mathrm{~K}$

Prism, clear light colourless

$0.28 \times 0.14 \times 0.06 \mathrm{~mm}$

8547 independent reflections

5499 reflections with $I>2 \sigma(I)$

$R_{\text {int }}=0.058$

$\theta_{\max }=68.7^{\circ}, \theta_{\min }=2.8^{\circ}$

$h=-7 \rightarrow 8$

$k=-44 \rightarrow 44$

$l=-21 \rightarrow 21$

$\mathrm{H}$ atoms treated by a mixture of independent

and constrained refinement

$w=1 /\left[\sigma^{2}\left(F_{\mathrm{o}}^{2}\right)+(0.0833 P)^{2}+2.1514 P\right]$

where $P=\left(F_{\mathrm{o}}{ }^{2}+2 F_{\mathrm{c}}{ }^{2}\right) / 3$

$(\Delta / \sigma)_{\max }=0.002$

$\Delta \rho_{\max }=0.27 \mathrm{e} \AA^{-3}$

$\Delta \rho_{\min }=-0.21 \mathrm{e} \AA^{-3}$

Extinction correction: SHELXL2018

(Sheldrick, 2015b),

$\mathrm{Fc}^{*}=\mathrm{kFc}\left[1+0.001 \mathrm{xFc}^{2} \lambda^{3} / \sin (2 \theta)\right]^{-1 / 4}$

Extinction coefficient: 0.00125 (13)

\section{Special details}

Geometry. All esds (except the esd in the dihedral angle between two 1.s. planes) are estimated using the full covariance matrix. The cell esds are taken into account individually in the estimation of esds in distances, angles and torsion angles; correlations between esds in cell parameters are only used when they are defined by crystal symmetry. An approximate (isotropic) treatment of cell esds is used for estimating esds involving l.s. planes.

Refinement. Single-crystal X-ray diffraction data collection ( scans and $\omega$ scans with $\kappa$ and $\theta$ offsets) were performed at room temperature on a Bruker AXS D8 VENTURE equipped with a Kappa goniometer, PHOTON II CPAD detector, a Mo $K \alpha$ INCOATEC I $\mu$ S 3.0 microfocus source $(\lambda=0.71073 \AA)$. The crystal centring, unit-cell determination, refinement of the unit-cell parameters and data collection was controlled through the program APEX3 (Bruker, 2012). The frame integration was performed using SAINT (Bruker, 2016) and the intensities were scaled and absorption corrected using SADABS (Bruker, 2001). Using OLEX2 (Dolomanov et al., 2009), the structure was solved by intrinsic phasing using SHELXT (Sheldrick, 2015) and refined by full-matrix least-squares calculation based on $F^{2}$ for all reflection using SHELXL (Sheldrick, 2007). All non-H atoms were refined anisotropically.

Fractional atomic coordinates and isotropic or equivalent isotropic displacement parameters $\left(\AA^{2}\right)$

\begin{tabular}{|c|c|c|c|c|c|}
\hline & $x$ & $y$ & $z$ & $U_{\text {iso }} * / U_{\text {eq }}$ & Occ. $(<1)$ \\
\hline $\mathrm{O} 1 \mathrm{C}$ & $0.8254(4)$ & $0.36199(7)$ & $-0.06761(14)$ & $0.0939(7)$ & \\
\hline $\mathrm{O} 2 \mathrm{C}$ & $0.5826(4)$ & $0.32866(7)$ & $-0.11345(12)$ & $0.0874(7)$ & \\
\hline $\mathrm{O} 3 \mathrm{C}$ & $0.3392(3)$ & $0.26359(5)$ & $0.03750(10)$ & $0.0688(5)$ & \\
\hline $\mathrm{O} 3 \mathrm{~A}$ & $0.7853(3)$ & $0.47087(5)$ & $0.86671(10)$ & $0.0634(5)$ & \\
\hline N1A & $0.9537(3)$ & $0.51097(6)$ & $0.74799(11)$ & $0.0540(5)$ & \\
\hline $\mathrm{O} 3 \mathrm{~B}$ & $0.7069(3)$ & $0.71873(5)$ & $0.49400(11)$ & $0.0704(5)$ & \\
\hline $\mathrm{O}^{\prime}$ & 0.5229 & $0.56376(5)$ & $0.34945(12)$ & $0.0766(6)$ & \\
\hline $\mathrm{O} 4^{\prime}$ & $0.8266(3)$ & $0.46389(5)$ & $0.49620(11)$ & $0.0721(6)$ & \\
\hline $\mathrm{O} 4 \mathrm{C}$ & $0.2932(4)$ & $0.20905(6)$ & $-0.01637(14)$ & $0.0909(7)$ & \\
\hline
\end{tabular}




\begin{tabular}{|c|c|c|c|c|c|}
\hline $\mathrm{N} 1 \mathrm{C}$ & $0.4294(3)$ & $0.33992(6)$ & $0.01710(12)$ & $0.0571(5)$ & \\
\hline $\mathrm{N} 2 \mathrm{C}$ & $0.5611(4)$ & $0.37320(7)$ & $0.11443(14)$ & $0.0772(7)$ & \\
\hline $\mathrm{N} 3 \mathrm{C}$ & $0.6713(4)$ & $0.34779(7)$ & $-0.06396(14)$ & $0.0668(6)$ & \\
\hline $\mathrm{C} 1 \mathrm{C}$ & $0.4139(5)$ & $0.35240(8)$ & $0.08614(16)$ & $0.0697(8)$ & \\
\hline $\mathrm{C} 2 \mathrm{C}$ & $0.6752(5)$ & $0.37470(8)$ & $0.06255(18)$ & $0.0705(8)$ & \\
\hline $\mathrm{H} 2 \mathrm{C}$ & $0.785(5)$ & $0.3879(9)$ & 0.0685 (17) & $0.074(9)^{*}$ & \\
\hline $\mathrm{C} 3 \mathrm{C}$ & $0.5974(4)$ & $0.35420(7)$ & $0.00232(15)$ & $0.0578(6)$ & \\
\hline $\mathrm{C} 4 \mathrm{C}$ & $0.2524(9)$ & $0.34396(14)$ & $0.1246(3)$ & $0.1032(14)$ & \\
\hline $\mathrm{H} 4 \mathrm{CA}$ & $0.207(7)$ & $0.3175(15)$ & $0.123(3)$ & $0.154(19)^{*}$ & \\
\hline $\mathrm{H} 4 \mathrm{CB}$ & $0.284(7)$ & $0.3513(15)$ & $0.170(3)$ & $0.15(2)^{*}$ & \\
\hline $\mathrm{H} 4 \mathrm{CC}$ & $0.140(10)$ & $0.3572(18)$ & $0.104(3)$ & $0.19(3)^{*}$ & \\
\hline $\mathrm{C} 5 \mathrm{C}$ & $0.2834(4)$ & $0.31870(8)$ & $-0.03207(18)$ & $0.0639(7)$ & \\
\hline H5CA & $0.260(4)$ & $0.3289(8)$ & $-0.0824(17)$ & $0.069(8)^{*}$ & \\
\hline H5CB & $0.161(5)$ & $0.3213(8)$ & $-0.0124(16)$ & $0.075(9)^{*}$ & \\
\hline C6C & $0.3292(5)$ & $0.27923(8)$ & $-0.03625(16)$ & $0.0651(7)$ & \\
\hline H6CA & $0.454(5)$ & $0.2757(9)$ & $-0.0532(18)$ & $0.085(10)^{*}$ & \\
\hline H6CB & $0.231(4)$ & $0.2679(8)$ & $-0.0697(16)$ & $0.063(8)^{*}$ & \\
\hline C7C & $0.3174(4)$ & $0.22757(8)$ & $0.03984(18)$ & $0.0659(7)$ & \\
\hline $\mathrm{C} 14 \mathrm{C}$ & $0.332(6)$ & $0.2182(8)$ & $0.1204(10)$ & $0.068(6)$ & 0.5318 \\
\hline $\mathrm{C} 15 \mathrm{C}$ & $0.390(4)$ & $0.2413(5)$ & $0.1819(15)$ & $0.068(3)$ & 0.5318 \\
\hline $\mathrm{H} 15 \mathrm{C}$ & 0.419867 & 0.265318 & 0.173166 & $0.082 *$ & 0.5318 \\
\hline $\mathrm{C} 16 \mathrm{C}$ & $0.403(2)$ & $0.2292(5)$ & $0.2561(9)$ & $0.093(4)$ & 0.5318 \\
\hline $\mathrm{H} 16 \mathrm{C}$ & 0.434781 & 0.245824 & 0.295117 & $0.112^{*}$ & 0.5318 \\
\hline $\mathrm{C} 17 \mathrm{C}$ & $0.370(2)$ & $0.1927(6)$ & $0.2740(8)$ & $0.112(5)$ & 0.5318 \\
\hline $\mathrm{H} 17 \mathrm{C}$ & 0.385648 & 0.184515 & 0.323553 & $0.135^{*}$ & 0.5318 \\
\hline $\mathrm{C} 18 \mathrm{C}$ & $0.310(2)$ & $0.1691(4)$ & $0.2119(7)$ & $0.101(6)$ & 0.5318 \\
\hline $\mathrm{H} 18 \mathrm{C}$ & 0.277796 & 0.145168 & 0.220181 & $0.121 *$ & 0.5318 \\
\hline C19C & $0.300(3)$ & $0.1817(6)$ & 0.1385 (13) & $0.088(4)$ & 0.5318 \\
\hline H19C & 0.270497 & 0.165123 & 0.099208 & $0.105^{*}$ & 0.5318 \\
\hline $\mathrm{C} 8 \mathrm{C}$ & $0.322(6)$ & $0.2090(9)$ & $0.1134(10)$ & $0.062(6)$ & 0.4682 \\
\hline $\mathrm{C} 13 \mathrm{C}$ & $0.364(4)$ & $0.2293(6)$ & $0.176(2)$ & $0.081(6)$ & 0.4682 \\
\hline $\mathrm{H} 13 \mathrm{C}$ & 0.392618 & 0.253879 & 0.173808 & $0.097^{*}$ & 0.4682 \\
\hline $\mathrm{C} 12 \mathrm{C}$ & $0.362(3)$ & $0.2123(5)$ & $0.2448(12)$ & $0.099(6)$ & 0.4682 \\
\hline $\mathrm{H} 12 \mathrm{C}$ & 0.390847 & 0.225357 & 0.289514 & $0.119^{*}$ & 0.4682 \\
\hline $\mathrm{C} 11 \mathrm{C}$ & $0.317(3)$ & $0.1770(6)$ & $0.2460(7)$ & $0.094(5)$ & 0.4682 \\
\hline $\mathrm{H} 11 \mathrm{C}$ & 0.317949 & 0.165177 & 0.291564 & $0.113^{*}$ & 0.4682 \\
\hline $\mathrm{C} 10 \mathrm{C}$ & $0.269(3)$ & $0.1580(6)$ & $0.1779(9)$ & $0.109(6)$ & 0.4682 \\
\hline $\mathrm{H} 10 \mathrm{C}$ & 0.238908 & 0.133490 & 0.179162 & $0.130 *$ & 0.4682 \\
\hline C9C & $0.266(4)$ & $0.1735(6)$ & $0.1132(13)$ & $0.081(5)$ & 0.4682 \\
\hline $\mathrm{H} 9 \mathrm{C}$ & 0.226439 & 0.160916 & 0.068431 & $0.097 *$ & 0.4682 \\
\hline O1A & $0.8622(4)$ & $0.60568(6)$ & $0.72428(15)$ & $0.1031(8)$ & \\
\hline $\mathrm{O} 2 \mathrm{~A}$ & $0.9845(4)$ & $0.57753(6)$ & $0.82685(13)$ & $0.0913(7)$ & \\
\hline $\mathrm{O} 4 \mathrm{~A}$ & $0.7325(4)$ & $0.47827(10)$ & 0.98395 (13) & $0.1178(10)$ & \\
\hline $\mathrm{N} 2 \mathrm{~A}$ & $0.8422(3)$ & $0.50522(6)$ & $0.62493(12)$ & $0.0589(5)$ & \\
\hline N3A & $0.9160(4)$ & $0.57788(7)$ & $0.75940(15)$ & $0.0722(7)$ & \\
\hline C1A & $0.9167(4)$ & $0.48799(7)$ & $0.68826(14)$ & $0.0548(6)$ & \\
\hline $\mathrm{C} 2 \mathrm{~A}$ & $0.8300(4)$ & $0.54068(8)$ & $0.64394(16)$ & $0.0613(7)$ & \\
\hline $\mathrm{H} 2 \mathrm{~A}$ & $0.782(4)$ & $0.5591(8)$ & $0.6103(18)$ & $0.076(9)^{*}$ & \\
\hline
\end{tabular}




\begin{tabular}{|c|c|c|c|c|}
\hline $\mathrm{C} 3 \mathrm{~A}$ & 0.8979 (4) & $0.54472(7)$ & $0.71862(15)$ & $0.0574(6)$ \\
\hline $\mathrm{C} 4 \mathrm{~A}$ & $0.9548(6)$ & $0.44854(9)$ & $0.6922(2)$ & $0.0726(8)$ \\
\hline H4AA & $1.089(6)$ & $0.4430(11)$ & $0.708(2)$ & $0.112(14)^{*}$ \\
\hline $\mathrm{H} 4 \mathrm{AB}$ & $0.892(5)$ & $0.4370(10)$ & $0.731(2)$ & $0.106(12)^{*}$ \\
\hline $\mathrm{H} 4 \mathrm{AC}$ & $0.902(6)$ & 0.4365 (11) & $0.648(3)$ & $0.122(14)^{*}$ \\
\hline $\mathrm{C} 5 \mathrm{~A}$ & $1.0501(4)$ & $0.50018(10)$ & $0.82398(15)$ & $0.0628(7)$ \\
\hline H5AA & $1.146(4)$ & $0.5180(8)$ & $0.8411(16)$ & $0.073(9)^{*}$ \\
\hline H5AB & $1.104(5)$ & $0.4746(10)$ & $0.8180(18)$ & $0.085(10)^{*}$ \\
\hline C6A & $0.9251(5)$ & $0.49971(10)$ & $0.88285(16)$ & $0.0666(7)$ \\
\hline H6AA & $1.011(4)$ & $0.4945(8)$ & $0.9322(18)$ & $0.078(9)^{*}$ \\
\hline H6AB & $0.859(5)$ & $0.5241(10)$ & $0.8861(18)$ & $0.091(11)^{*}$ \\
\hline C7A & $0.6892(4)$ & $0.46458(9)$ & $0.92272(16)$ & $0.0726(8)$ \\
\hline C8A & $0.5226(4)$ & $0.44060(9)$ & $0.90204(17)$ & $0.0701(8)$ \\
\hline C9A & $0.4877(5)$ & $0.42069(9)$ & $0.8363(2)$ & $0.0777(9)$ \\
\hline H9A & $0.580(4)$ & $0.4208(8)$ & $0.8021(17)$ & $0.072(9)^{*}$ \\
\hline C10A & $0.3229(6)$ & $0.39958(10)$ & $0.8191(3)$ & 0.0967 (13) \\
\hline $\mathrm{H} 10 \mathrm{~A}$ & $0.307(6)$ & $0.3863(11)$ & $0.770(2)$ & $0.117(14)^{*}$ \\
\hline C11A & $0.1962(6)$ & $0.39897(12)$ & $0.8675(3)$ & $0.1073(15)$ \\
\hline H11A & $0.073(7)$ & $0.3852(12)$ & $0.856(3)$ & $0.135(16)^{*}$ \\
\hline $\mathrm{C} 12 \mathrm{~A}$ & $0.2295(6)$ & $0.41829(12)$ & $0.9333(3)$ & $0.1003(13)$ \\
\hline $\mathrm{H} 12 \mathrm{~A}$ & $0.134(6)$ & $0.4175(11)$ & $0.969(2)$ & $0.115(13)^{*}$ \\
\hline C13A & $0.3934(5)$ & 0.43885 (11) & $0.9514(2)$ & $0.0867(10)$ \\
\hline H13A & $0.422(6)$ & $0.4556(11)$ & $0.993(2)$ & $0.107(14)^{*}$ \\
\hline O1B & $0.2377(4)$ & $0.64048(6)$ & $0.62852(13)$ & $0.0840(6)$ \\
\hline $\mathrm{O} 2 \mathrm{~B}$ & $0.4848(4)$ & $0.67270(7)$ & $0.67579(13)$ & $0.0956(8)$ \\
\hline O4B & $0.6319(4)$ & $0.77703(6)$ & $0.50557(12)$ & $0.0850(7)$ \\
\hline N1B & $0.6714(3)$ & $0.64843(6)$ & $0.56085(12)$ & $0.0595(5)$ \\
\hline N2B & $0.5676(4)$ & $0.60475(6)$ & $0.47891(13)$ & $0.0670(6)$ \\
\hline N3B & $0.4023(4)$ & $0.65101(7)$ & 0.62963 (14) & $0.0713(7)$ \\
\hline $\mathrm{C} 1 \mathrm{~B}$ & $0.7086(4)$ & $0.62802(7)$ & $0.50247(16)$ & $0.0645(7)$ \\
\hline $\mathrm{C} 2 \mathrm{~B}$ & $0.4352(5)$ & $0.61020(8)$ & $0.52319(17)$ & $0.0656(7)$ \\
\hline $\mathrm{H} 2 \mathrm{~B}$ & $0.320(4)$ & $0.5970(8)$ & $0.5189(16)$ & $0.066(8)^{*}$ \\
\hline C3B & $0.4962(4)$ & $0.63698(7)$ & $0.57283(15)$ & $0.0596(7)$ \\
\hline C4B & $0.8810(5)$ & $0.63092(11)$ & $0.4683(2)$ & 0.0961 (11) \\
\hline H4BA & 0.890475 & 0.655102 & 0.449353 & $0.144 *$ \\
\hline H4BB & 0.873150 & 0.613816 & 0.427700 & $0.144^{*}$ \\
\hline $\mathrm{H} 4 \mathrm{BC}$ & 0.991440 & 0.625719 & 0.505677 & $0.144^{*}$ \\
\hline $\mathrm{C} 5 \mathrm{~B}$ & $0.7922(5)$ & $0.67798(8)$ & $0.5974(2)$ & $0.0694(8)$ \\
\hline H5BA & $0.807(5)$ & $0.6749(9)$ & $0.652(2)$ & $0.087(10)^{*}$ \\
\hline H5BB & $0.912(5)$ & $0.6750(8)$ & $0.5815(16)$ & $0.072(9)^{*}$ \\
\hline C6B & $0.7111(5)$ & $0.71471(8)$ & $0.57400(17)$ & $0.0683(8)$ \\
\hline H6BA & $0.786(4)$ & $0.7336(8)$ & $0.6008(16)$ & $0.063(8)^{*}$ \\
\hline H6BB & $0.574(5)$ & $0.7165(8)$ & $0.5825(17)$ & $0.078(9)^{*}$ \\
\hline C7B & $0.6616(4)$ & $0.75204(8)$ & $0.46585(16)$ & $0.0633(7)$ \\
\hline $\mathrm{C} 8 \mathrm{~B}$ & $0.6546(4)$ & $0.75459(8)$ & $0.38362(16)$ & $0.0656(7)$ \\
\hline C9B & $0.6406(5)$ & $0.72461(11)$ & $0.3370(2)$ & $0.0789(9)$ \\
\hline H9B & $0.642(4)$ & $0.7020(8)$ & $0.3596(16)$ & $0.065(9)^{*}$ \\
\hline $\mathrm{C} 10 \mathrm{~B}$ & $0.6313(6)$ & $0.72971(15)$ & $0.2597(2)$ & $0.0979(12)$ \\
\hline
\end{tabular}




$\begin{array}{lllll}\mathrm{H} 10 \mathrm{~B} & 0.631(5) & 0.7072(11) & 0.233(2) & 0.111(13)^{*} \\ \mathrm{C} 11 \mathrm{~B} & 0.6431(6) & 0.76410(15) & 0.2314(2) & 0.1012(12) \\ \mathrm{H} 11 \mathrm{~B} & 0.643(6) & 0.7687(12) & 0.175(3) & 0.139(16)^{*} \\ \mathrm{C} 12 \mathrm{~B} & 0.6565(6) & 0.79407(14) & 0.2768(2) & 0.0951(11) \\ \mathrm{H} 12 \mathrm{~B} & 0.670(7) & 0.8222(16) & 0.256(3) & 0.17(2)^{*} \\ \mathrm{C} 13 \mathrm{~B} & 0.6609(5) & 0.78897(11) & 0.3529(2) & 0.0802(9) \\ \mathrm{H} 13 \mathrm{~B} & 0.664(5) & 0.8100(9) & 0.3852(19) & 0.082(10)^{*} \\ \mathrm{O} 1^{\prime} & 0.6210(4) & 0.41366(6) & 0.24229(12) & 0.0924(8) \\ \mathrm{H} 1^{\prime} & 0.593(6) & 0.4002(12) & 0.194(3) & 0.130(15)^{*} \\ \mathrm{O}^{\prime} & 0.4848(4) & 0.45805(6) & 0.17062(11) & 0.0929(8) \\ \mathrm{H}^{\prime} & 0.540(5) & 0.5769(11) & 0.397(2) & 0.111(13)^{*} \\ \mathrm{H}^{\prime} & 0.829(6) & 0.4799(11) & 0.538(2) & 0.118(13)^{*} \\ \mathrm{C} 1^{\prime} & 0.6075(3) & 0.47064(6) & 0.29968(12) & 0.0478(5) \\ \mathrm{C} 2^{\prime} & 0.5473(4) & 0.50641(7) & 0.29381(14) & 0.0538(6) \\ \mathrm{H} 2^{\prime} & 0.485(4) & 0.5175(8) & 0.2465(16) & 0.066(8)^{*} \\ \mathrm{C}^{\prime} & 0.5826(4) & 0.52839(7) & 0.35727(14) & 0.0547(6) \\ \mathrm{C}^{\prime} & 0.6741(4) & 0.51454(7) & 0.42558(14) & 0.0547(6) \\ \mathrm{H}^{\prime} \mathrm{A} & 0.695(4) & 0.5297(8) & 0.4690(16) & 0.066(8)^{*} \\ \mathrm{C}^{\prime} & 0.7340(4) & 0.47867(7) & 0.43047(13) & 0.0538(6) \\ \mathrm{C}^{\prime} & 0.7015(4) & 0.45642(7) & 0.36715(14) & 0.0525(6) \\ \mathrm{H}^{\prime} & 0.745(4) & 0.4308(7) & 0.3716(14) & 0.056(7)^{*} \\ \mathrm{C}^{\prime} & 0.5633(4) & 0.44730(7) & 0.23118(14) & 0.0547(6)\end{array}$

Atomic displacement parameters $\left(\AA^{2}\right)$

\begin{tabular}{lllllll}
\hline & $U^{11}$ & $U^{22}$ & $U^{33}$ & $U^{12}$ & $U^{13}$ & $U^{23}$ \\
\hline O1C & $0.0824(16)$ & $0.1000(17)$ & $0.1057(18)$ & $-0.0177(14)$ & $0.0339(14)$ & $0.0013(13)$ \\
O2C & $0.0938(16)$ & $0.1079(17)$ & $0.0617(12)$ & $-0.0126(14)$ & $0.0174(12)$ & $-0.0190(12)$ \\
O3C & $0.0881(14)$ & $0.0579(11)$ & $0.0581(11)$ & $-0.0031(10)$ & $0.0070(10)$ & $-0.0047(8)$ \\
O3A & $0.0616(11)$ & $0.0758(12)$ & $0.0500(10)$ & $-0.0110(9)$ & $0.0026(8)$ & $0.0045(8)$ \\
N1A & $0.0514(12)$ & $0.0630(12)$ & $0.0460(11)$ & $-0.0034(10)$ & $0.0046(9)$ & $0.0001(9)$ \\
O3B & $0.0864(14)$ & $0.0615(11)$ & $0.0644(12)$ & $-0.0020(10)$ & $0.0164(10)$ & $-0.0104(9)$ \\
O3' & $0.1090(17)$ & $0.0550(11)$ & $0.0595(12)$ & $0.0166(11)$ & $-0.0012(11)$ & $-0.0068(9)$ \\
O4' & $0.0980(16)$ & $0.0601(11)$ & $0.0497(11)$ & $0.0065(10)$ & $-0.0089(10)$ & $-0.0005(9)$ \\
O4C & $0.116(2)$ & $0.0611(12)$ & $0.0930(16)$ & $-0.0062(12)$ & $0.0128(14)$ & $-0.0160(11)$ \\
N1C & $0.0628(14)$ & $0.0536(12)$ & $0.0546(12)$ & $0.0006(10)$ & $0.0095(10)$ & $-0.0075(9)$ \\
N2C & $0.104(2)$ & $0.0613(14)$ & $0.0633(15)$ & $0.0020(14)$ & $0.0076(15)$ & $-0.0174(11)$ \\
N3C & $0.0673(16)$ & $0.0680(14)$ & $0.0659(15)$ & $-0.0002(12)$ & $0.0147(12)$ & $0.0021(12)$ \\
C1C & $0.090(2)$ & $0.0590(16)$ & $0.0627(17)$ & $0.0081(16)$ & $0.0208(16)$ & $-0.0108(13)$ \\
C2C & $0.080(2)$ & $0.0572(16)$ & $0.0690(19)$ & $-0.0039(16)$ & $0.0005(16)$ & $-0.0073(13)$ \\
C3C & $0.0616(16)$ & $0.0537(14)$ & $0.0570(15)$ & $-0.0024(12)$ & $0.0075(12)$ & $-0.0062(11)$ \\
C4C & $0.121(4)$ & $0.102(3)$ & $0.103(3)$ & $0.003(3)$ & $0.062(3)$ & $-0.019(3)$ \\
C5C & $0.0621(18)$ & $0.0632(16)$ & $0.0633(17)$ & $-0.0010(14)$ & $0.0028(14)$ & $-0.0020(13)$ \\
C6C & $0.077(2)$ & $0.0599(16)$ & $0.0555(16)$ & $-0.0066(15)$ & $0.0039(15)$ & $-0.0061(12)$ \\
C7C & $0.0581(17)$ & $0.0605(16)$ & $0.0777(19)$ & $0.0021(13)$ & $0.0089(14)$ & $0.0006(14)$ \\
C14C & $0.056(6)$ & $0.054(13)$ & $0.098(10)$ & $0.007(8)$ & $0.024(7)$ & $0.010(8)$ \\
C15C & $0.065(7)$ & $0.081(9)$ & $0.059(5)$ & $-0.005(7)$ & $0.013(5)$ & $0.002(7)$ \\
C16C & $0.074(6)$ & $0.136(13)$ & $0.072(8)$ & $-0.007(7)$ & $0.021(5)$ & $0.011(7)$ \\
& & & & & &
\end{tabular}




\begin{tabular}{|c|c|c|c|c|c|c|}
\hline $\mathrm{C} 17 \mathrm{C}$ & $0.083(8)$ & $0.183(16)$ & $0.073(10)$ & $0.020(8)$ & $0.019(7)$ & $0.038(9)$ \\
\hline $\mathrm{C} 18 \mathrm{C}$ & $0.126(9)$ & $0.123(15)$ & $0.063(13)$ & $0.014(9)$ & $0.043(12)$ & 0.038 (11) \\
\hline $\mathrm{C} 19 \mathrm{C}$ & $0.084(7)$ & $0.068(10)$ & 0.114 (14) & $0.003(6)$ & $0.027(8)$ & $0.007(8)$ \\
\hline $\mathrm{C} 8 \mathrm{C}$ & $0.066(8)$ & $0.054(15)$ & 0.067 (6) & $0.011(10)$ & $0.017(5)$ & $0.005(6)$ \\
\hline $\mathrm{C} 13 \mathrm{C}$ & $0.070(9)$ & 0.087 (13) & $0.086(10)$ & -0.007 (9) & $0.015(8)$ & $0.011(13)$ \\
\hline $\mathrm{C} 12 \mathrm{C}$ & $0.094(12)$ & $0.14(2)$ & $0.063(8)$ & $0.015(11)$ & $0.010(7)$ & $-0.002(10)$ \\
\hline $\mathrm{C} 11 \mathrm{C}$ & $0.093(9)$ & $0.155(15)$ & $0.036(10)$ & $0.032(8)$ & $0.021(8)$ & $0.029(10)$ \\
\hline $\mathrm{C} 10 \mathrm{C}$ & $0.150(14)$ & $0.101(10)$ & $0.088(13)$ & $0.036(9)$ & $0.056(11)$ & $0.020(8)$ \\
\hline C9C & $0.100(12)$ & $0.058(9)$ & 0.095 (11) & $0.005(7)$ & $0.044(10)$ & $0.007(6)$ \\
\hline O1A & $0.137(2)$ & $0.0657(14)$ & 0.1079 (19) & $0.0135(14)$ & $0.0244(17)$ & $-0.0030(13)$ \\
\hline $\mathrm{O} 2 \mathrm{~A}$ & 0.1095 (19) & $0.0871(15)$ & $0.0741(15)$ & $-0.0183(13)$ & $0.0079(13)$ & $-0.0214(11)$ \\
\hline $\mathrm{O} 4 \mathrm{~A}$ & 0.1005 (19) & $0.196(3)$ & $0.0587(14)$ & $-0.052(2)$ & $0.0189(13)$ & $-0.0193(16)$ \\
\hline $\mathrm{N} 2 \mathrm{~A}$ & 0.0597 (13) & $0.0659(13)$ & $0.0497(12)$ & $-0.0009(11)$ & $0.0058(10)$ & $0.0008(10)$ \\
\hline N3A & $0.0755(17)$ & $0.0693(16)$ & $0.0738(17)$ & $-0.0062(13)$ & $0.0188(14)$ & $-0.0087(13)$ \\
\hline $\mathrm{C} 1 \mathrm{~A}$ & $0.0532(15)$ & $0.0615(15)$ & $0.0478(13)$ & $-0.0055(12)$ & $0.0042(11)$ & $-0.0006(11)$ \\
\hline $\mathrm{C} 2 \mathrm{~A}$ & $0.0597(17)$ & $0.0686(17)$ & $0.0553(15)$ & $0.0032(14)$ & 0.0094 (13) & $0.0067(13)$ \\
\hline $\mathrm{C} 3 \mathrm{~A}$ & $0.0550(15)$ & $0.0584(15)$ & $0.0587(15)$ & $-0.0015(12)$ & $0.0102(12)$ & $-0.0027(12)$ \\
\hline $\mathrm{C} 4 \mathrm{~A}$ & $0.083(2)$ & $0.0656(18)$ & $0.0656(19)$ & $-0.0013(17)$ & $0.0029(18)$ & $-0.0013(15)$ \\
\hline $\mathrm{C} 5 \mathrm{~A}$ & $0.0545(16)$ & $0.082(2)$ & $0.0474(14)$ & $-0.0103(16)$ & $-0.0024(12)$ & $0.0011(13)$ \\
\hline C6A & $0.0629(18)$ & $0.084(2)$ & $0.0497(15)$ & $-0.0158(16)$ & $0.0029(13)$ & $-0.0046(13)$ \\
\hline C7A & $0.0642(18)$ & $0.099(2)$ & $0.0526(16)$ & $-0.0075(16)$ & $0.0065(14)$ & $0.0075(15)$ \\
\hline $\mathrm{C} 8 \mathrm{~A}$ & $0.0601(17)$ & 0.0775 (19) & $0.0684(18)$ & $-0.0014(15)$ & $0.0002(14)$ & $0.0213(15)$ \\
\hline C9A & $0.075(2)$ & $0.0714(19)$ & $0.080(2)$ & $-0.0069(17)$ & $-0.0042(18)$ & $0.0225(16)$ \\
\hline $\mathrm{C} 10 \mathrm{~A}$ & $0.096(3)$ & $0.073(2)$ & $0.106(3)$ & $-0.017(2)$ & $-0.022(2)$ & $0.025(2)$ \\
\hline $\mathrm{C} 11 \mathrm{~A}$ & 0.075 & 0.091 (3) & $0.147(4)$ & $-0.015(2)$ & $-0.004(3)$ & $0.054(3)$ \\
\hline $\mathrm{C} 12 \mathrm{~A}$ & $0.070(2)$ & 0.098 & $0.132(4)$ & $0.000(2)$ & $0.016(3)$ & 0.048 \\
\hline $\mathrm{C} 13 \mathrm{~A}$ & $0.072(2)$ & $0.089(2)$ & $0.100(3)$ & 0.0007 (19) & $0.018(2)$ & $0.030(2)$ \\
\hline O1B & $0.0854(16)$ & $0.0831(14)$ & $0.0907(16)$ & $0.0068(12)$ & $0.0346(13)$ & $0.0075(11)$ \\
\hline $\mathrm{O} 2 \mathrm{~B}$ & $0.119(2)$ & $0.0971(17)$ & $0.0754(14)$ & $-0.0060(15)$ & $0.0307(14)$ & $-0.0279(13)$ \\
\hline O4B & $0.1183(19)$ & $0.0642(12)$ & $0.0757(13)$ & $0.0054(12)$ & $0.0259(13)$ & $-0.0099(10)$ \\
\hline N1B & $0.0624(14)$ & $0.0547(12)$ & $0.0617(13)$ & $0.0001(10)$ & $0.0124(11)$ & $-0.0074(10)$ \\
\hline N2B & $0.0788(16)$ & 0.0565 (13) & 0.0677 (14) & $0.0021(12)$ & $0.0185(12)$ & $-0.0091(11)$ \\
\hline N3B & 0.0839 (19) & $0.0675(15)$ & $0.0669(15)$ & 0.0051 (14) & $0.0255(13)$ & $0.0013(12)$ \\
\hline C1B & $0.0701(18)$ & $0.0575(15)$ & $0.0682(17)$ & $0.0022(14)$ & $0.0184(14)$ & $-0.0096(13)$ \\
\hline $\mathrm{C} 2 \mathrm{~B}$ & $0.073(2)$ & $0.0566(15)$ & $0.0691(18)$ & $0.0000(15)$ & $0.0184(15)$ & $-0.0025(13)$ \\
\hline $\mathrm{C} 3 \mathrm{~B}$ & $0.0700(18)$ & $0.0549(14)$ & $0.0556(15)$ & $0.0033(13)$ & $0.0160(13)$ & $-0.0023(11)$ \\
\hline C4B & $0.082(2)$ & $0.108(3)$ & $0.107(3)$ & $-0.003(2)$ & $0.040(2)$ & $-0.027(2)$ \\
\hline $\mathrm{C} 5 \mathrm{~B}$ & $0.071(2)$ & $0.0659(17)$ & $0.068(2)$ & $-0.0021(15)$ & $0.0047(16)$ & $-0.0121(14)$ \\
\hline C6B & $0.082(2)$ & $0.0601(16)$ & $0.0617(17)$ & $-0.0084(16)$ & $0.0107(15)$ & $-0.0123(13)$ \\
\hline C7B & $0.0599(17)$ & $0.0608(16)$ & $0.0692(17)$ & $-0.0066(13)$ & $0.0118(14)$ & $-0.0101(13)$ \\
\hline $\mathrm{C} 8 \mathrm{~B}$ & $0.0540(16)$ & $0.0779(19)$ & $0.0640(17)$ & $-0.0071(14)$ & $0.0081(13)$ & $-0.0126(14)$ \\
\hline C9B & $0.070(2)$ & $0.087(2)$ & $0.079(2)$ & $-0.0118(18)$ & $0.0120(16)$ & $-0.0207(18)$ \\
\hline $\mathrm{C} 10 \mathrm{~B}$ & 0.083 & $0.130(4)$ & $0.079(2)$ & $-0.007(2)$ & $0.0084(19)$ & $-0.038(3)$ \\
\hline $\mathrm{C} 11 \mathrm{~B}$ & 0.085 (3) & $0.147(4)$ & $0.071(2)$ & $0.002(3)$ & $0.011(2)$ & $0.002(3)$ \\
\hline C12B & $0.092(3)$ & $0.120(3)$ & $0.073(2)$ & $0.000(2)$ & 0.0145 (19) & $0.010(2)$ \\
\hline C13B & 0.079 (2) & $0.085(2)$ & $0.076(2)$ & $-0.0051(18)$ & $0.0126(17)$ & $-0.0013(18)$ \\
\hline $\mathrm{O} 1^{\prime}$ & $0.151(2)$ & $0.0591(12)$ & $0.0587(12)$ & $0.0135(13)$ & $-0.0021(13)$ & $-0.0159(10)$ \\
\hline $\mathrm{O} 2^{\prime}$ & $0.130(2)$ & $0.0837(15)$ & $0.0529(12)$ & $0.0226(14)$ & $-0.0171(12)$ & $-0.0174(10)$ \\
\hline
\end{tabular}




\begin{tabular}{lllllll}
$\mathrm{C}^{\prime}$ & $0.0488(13)$ & $0.0526(13)$ & $0.0419(12)$ & $-0.0064(11)$ & $0.0078(10)$ & $-0.0060(10)$ \\
$\mathrm{C}^{\prime}$ & $0.0589(16)$ & $0.0548(14)$ & $0.0457(13)$ & $-0.0004(12)$ & $0.0038(11)$ & $-0.0022(11)$ \\
$\mathrm{C}^{\prime}$ & $0.0602(16)$ & $0.0490(13)$ & $0.0540(14)$ & $0.0010(12)$ & $0.0083(12)$ & $-0.0047(11)$ \\
$\mathrm{C}^{\prime}$ & $0.0638(16)$ & $0.0545(14)$ & $0.0430(13)$ & $-0.0027(12)$ & $0.0020(12)$ & $-0.0084(11)$ \\
$\mathrm{C}^{\prime}$ & $0.0581(15)$ & $0.0565(14)$ & $0.0441(13)$ & $-0.0025(12)$ & $0.0023(11)$ & $-0.0014(10)$ \\
$\mathrm{C}^{\prime}$ & $0.0576(15)$ & $0.0489(14)$ & $0.0498(13)$ & $-0.0013(12)$ & $0.0067(11)$ & $-0.0038(10)$ \\
$\mathrm{C}^{\prime}$ & $0.0569(15)$ & $0.0583(15)$ & $0.0486(14)$ & $-0.0037(12)$ & $0.0084(12)$ & $-0.0073(11)$ \\
\hline
\end{tabular}

Geometric parameters $\left(\AA,{ }^{\circ}\right)$

\begin{tabular}{|c|c|c|c|}
\hline $\mathrm{O} 1 \mathrm{C}-\mathrm{N} 3 \mathrm{C}$ & $1.232(3)$ & $\mathrm{C} 4 \mathrm{~A}-\mathrm{H} 4 \mathrm{AA}$ & $0.97(4)$ \\
\hline $\mathrm{O} 2 \mathrm{C}-\mathrm{N} 3 \mathrm{C}$ & $1.221(3)$ & $\mathrm{C} 4 \mathrm{~A}-\mathrm{H} 4 \mathrm{AB}$ & $1.00(4)$ \\
\hline $\mathrm{O} 3 \mathrm{C}-\mathrm{C} 6 \mathrm{C}$ & $1.440(3)$ & $\mathrm{C} 4 \mathrm{~A}-\mathrm{H} 4 \mathrm{AC}$ & $0.94(5)$ \\
\hline $\mathrm{O} 3 \mathrm{C}-\mathrm{C} 7 \mathrm{C}$ & $1.336(3)$ & $\mathrm{C} 5 \mathrm{~A}-\mathrm{H} 5 \mathrm{AA}$ & $0.96(3)$ \\
\hline $\mathrm{O} 3 \mathrm{~A}-\mathrm{C} 6 \mathrm{~A}$ & $1.450(3)$ & $\mathrm{C} 5 \mathrm{~A}-\mathrm{H} 5 \mathrm{AB}$ & $1.03(3)$ \\
\hline $\mathrm{O} 3 \mathrm{~A}-\mathrm{C} 7 \mathrm{~A}$ & $1.342(3)$ & $\mathrm{C} 5 \mathrm{~A}-\mathrm{C} 6 \mathrm{~A}$ & $1.508(4)$ \\
\hline $\mathrm{N} 1 \mathrm{~A}-\mathrm{C} 1 \mathrm{~A}$ & $1.358(3)$ & C6A-H6AA & $1.00(3)$ \\
\hline $\mathrm{N} 1 \mathrm{~A}-\mathrm{C} 3 \mathrm{~A}$ & $1.380(3)$ & C6A-H6AB & $1.02(4)$ \\
\hline $\mathrm{N} 1 \mathrm{~A}-\mathrm{C} 5 \mathrm{~A}$ & $1.474(3)$ & $\mathrm{C} 7 \mathrm{~A}-\mathrm{C} 8 \mathrm{~A}$ & $1.475(4)$ \\
\hline $\mathrm{O} 3 \mathrm{~B}-\mathrm{C} 6 \mathrm{~B}$ & $1.447(3)$ & $\mathrm{C} 8 \mathrm{~A}-\mathrm{C} 9 \mathrm{~A}$ & $1.379(5)$ \\
\hline $\mathrm{O} 3 \mathrm{~B}-\mathrm{C} 7 \mathrm{~B}$ & $1.344(3)$ & $\mathrm{C} 8 \mathrm{~A}-\mathrm{C} 13 \mathrm{~A}$ & $1.396(5)$ \\
\hline $\mathrm{O} 3^{\prime}-\mathrm{H} 3^{\prime}$ & $0.97(4)$ & $\mathrm{C} 9 \mathrm{~A}-\mathrm{H} 9 \mathrm{~A}$ & $0.98(3)$ \\
\hline $\mathrm{O}^{\prime}-\mathrm{C} 3^{\prime}$ & $1.369(3)$ & $\mathrm{C} 9 \mathrm{~A}-\mathrm{C} 10 \mathrm{~A}$ & $1.398(5)$ \\
\hline $\mathrm{O} 4^{\prime}-\mathrm{H} 4^{\prime}$ & $0.96(4)$ & $\mathrm{C} 10 \mathrm{~A}-\mathrm{H} 10 \mathrm{~A}$ & $0.99(4)$ \\
\hline $\mathrm{O} 4^{\prime}-\mathrm{C} 5^{\prime}$ & $1.363(3)$ & $\mathrm{C} 10 \mathrm{~A}-\mathrm{C} 11 \mathrm{~A}$ & $1.368(7)$ \\
\hline $\mathrm{O} 4 \mathrm{C}-\mathrm{C} 7 \mathrm{C}$ & 1.209 (4) & $\mathrm{C} 11 \mathrm{~A}-\mathrm{H} 11 \mathrm{~A}$ & $1.00(5)$ \\
\hline $\mathrm{N} 1 \mathrm{C}-\mathrm{C} 1 \mathrm{C}$ & $1.352(3)$ & $\mathrm{C} 11 \mathrm{~A}-\mathrm{C} 12 \mathrm{~A}$ & $1.368(7)$ \\
\hline $\mathrm{N} 1 \mathrm{C}-\mathrm{C} 3 \mathrm{C}$ & $1.381(3)$ & $\mathrm{C} 12 \mathrm{~A}-\mathrm{H} 12 \mathrm{~A}$ & $1.03(4)$ \\
\hline $\mathrm{N} 1 \mathrm{C}-\mathrm{C} 5 \mathrm{C}$ & $1.466(3)$ & $\mathrm{C} 12 \mathrm{~A}-\mathrm{C} 13 \mathrm{~A}$ & $1.382(6)$ \\
\hline $\mathrm{N} 2 \mathrm{C}-\mathrm{C} 1 \mathrm{C}$ & $1.326(4)$ & $\mathrm{C} 13 \mathrm{~A}-\mathrm{H} 13 \mathrm{~A}$ & $0.96(4)$ \\
\hline $\mathrm{N} 2 \mathrm{C}-\mathrm{C} 2 \mathrm{C}$ & $1.350(4)$ & $\mathrm{O} 1 \mathrm{~B}-\mathrm{N} 3 \mathrm{~B}$ & $1.235(3)$ \\
\hline $\mathrm{N} 3 \mathrm{C}-\mathrm{C} 3 \mathrm{C}$ & $1.413(3)$ & $\mathrm{O} 2 \mathrm{~B}-\mathrm{N} 3 \mathrm{~B}$ & $1.225(3)$ \\
\hline $\mathrm{C} 1 \mathrm{C}-\mathrm{C} 4 \mathrm{C}$ & $1.484(5)$ & $\mathrm{O} 4 \mathrm{~B}-\mathrm{C} 7 \mathrm{~B}$ & $1.208(3)$ \\
\hline $\mathrm{C} 2 \mathrm{C}-\mathrm{H} 2 \mathrm{C}$ & $0.92(3)$ & $\mathrm{N} 1 \mathrm{~B}-\mathrm{C} 1 \mathrm{~B}$ & $1.359(3)$ \\
\hline $\mathrm{C} 2 \mathrm{C}-\mathrm{C} 3 \mathrm{C}$ & $1.357(4)$ & $\mathrm{N} 1 \mathrm{~B}-\mathrm{C} 3 \mathrm{~B}$ & $1.375(3)$ \\
\hline $\mathrm{C} 4 \mathrm{C}-\mathrm{H} 4 \mathrm{CA}$ & $1.02(5)$ & $\mathrm{N} 1 \mathrm{~B}-\mathrm{C} 5 \mathrm{~B}$ & $1.468(4)$ \\
\hline $\mathrm{C} 4 \mathrm{C}-\mathrm{H} 4 \mathrm{CB}$ & $0.85(6)$ & $\mathrm{N} 2 \mathrm{~B}-\mathrm{C} 1 \mathrm{~B}$ & $1.332(4)$ \\
\hline $\mathrm{C} 4 \mathrm{C}-\mathrm{H} 4 \mathrm{CC}$ & $0.95(7)$ & $\mathrm{N} 2 \mathrm{~B}-\mathrm{C} 2 \mathrm{~B}$ & $1.360(4)$ \\
\hline $\mathrm{C} 5 \mathrm{C}-\mathrm{H} 5 \mathrm{CA}$ & $0.97(3)$ & $\mathrm{N} 3 \mathrm{~B}-\mathrm{C} 3 \mathrm{~B}$ & $1.421(3)$ \\
\hline $\mathrm{C} 5 \mathrm{C}-\mathrm{H} 5 \mathrm{CB}$ & $1.01(3)$ & $\mathrm{C} 1 \mathrm{~B}-\mathrm{C} 4 \mathrm{~B}$ & $1.479(4)$ \\
\hline $\mathrm{C} 5 \mathrm{C}-\mathrm{C} 6 \mathrm{C}$ & $1.493(4)$ & $\mathrm{C} 2 \mathrm{~B}-\mathrm{H} 2 \mathrm{~B}$ & $0.95(3)$ \\
\hline $\mathrm{C} 6 \mathrm{C}-\mathrm{H} 6 \mathrm{CA}$ & $1.00(3)$ & $\mathrm{C} 2 \mathrm{~B}-\mathrm{C} 3 \mathrm{~B}$ & $1.350(4)$ \\
\hline $\mathrm{C} 6 \mathrm{C}-\mathrm{H} 6 \mathrm{CB}$ & $0.94(3)$ & $\mathrm{C} 4 \mathrm{~B}-\mathrm{H} 4 \mathrm{BA}$ & 0.9600 \\
\hline $\mathrm{C} 7 \mathrm{C}-\mathrm{C} 14 \mathrm{C}$ & $1.480(13)$ & $\mathrm{C} 4 \mathrm{~B}-\mathrm{H} 4 \mathrm{BB}$ & 0.9600 \\
\hline $\mathrm{C} 7 \mathrm{C}-\mathrm{C} 8 \mathrm{C}$ & 1.489 (12) & $\mathrm{C} 4 \mathrm{~B}-\mathrm{H} 4 \mathrm{BC}$ & 0.9600 \\
\hline $\mathrm{C} 14 \mathrm{C}-\mathrm{C} 15 \mathrm{C}$ & $1.40(3)$ & $\mathrm{C} 5 \mathrm{~B}-\mathrm{H} 5 \mathrm{BA}$ & $0.98(3)$ \\
\hline $\mathrm{C} 14 \mathrm{C}-\mathrm{C} 19 \mathrm{C}$ & $1.41(3)$ & $\mathrm{C} 5 \mathrm{~B}-\mathrm{H} 5 \mathrm{BB}$ & $0.96(3)$ \\
\hline $\mathrm{C} 15 \mathrm{C}-\mathrm{H} 15 \mathrm{C}$ & 0.9300 & $\mathrm{C} 5 \mathrm{~B}-\mathrm{C} 6 \mathrm{~B}$ & $1.500(4)$ \\
\hline
\end{tabular}




\begin{tabular}{|c|c|c|c|}
\hline $\mathrm{C} 15 \mathrm{C}-\mathrm{C} 16 \mathrm{C}$ & $1.40(3)$ & $\mathrm{C} 6 \mathrm{~B}-\mathrm{H} 6 \mathrm{BA}$ & $0.95(3)$ \\
\hline $\mathrm{C} 16 \mathrm{C}-\mathrm{H} 16 \mathrm{C}$ & 0.9300 & C6B-H6BB & $1.02(3)$ \\
\hline $\mathrm{C} 16 \mathrm{C}-\mathrm{C} 17 \mathrm{C}$ & $1.41(3)$ & $\mathrm{C} 7 \mathrm{~B}-\mathrm{C} 8 \mathrm{~B}$ & $1.480(4)$ \\
\hline $\mathrm{C} 17 \mathrm{C}-\mathrm{H} 17 \mathrm{C}$ & 0.9300 & $\mathrm{C} 8 \mathrm{~B}-\mathrm{C} 9 \mathrm{~B}$ & $1.380(4)$ \\
\hline $\mathrm{C} 17 \mathrm{C}-\mathrm{C} 18 \mathrm{C}$ & $1.42(2)$ & $\mathrm{C} 8 \mathrm{~B}-\mathrm{C} 13 \mathrm{~B}$ & $1.386(5)$ \\
\hline $\mathrm{C} 18 \mathrm{C}-\mathrm{H} 18 \mathrm{C}$ & 0.9300 & $\mathrm{C} 9 \mathrm{~B}-\mathrm{H} 9 \mathrm{~B}$ & $0.93(3)$ \\
\hline $\mathrm{C} 18 \mathrm{C}-\mathrm{C} 19 \mathrm{C}$ & $1.39(3)$ & $\mathrm{C} 9 \mathrm{~B}-\mathrm{C} 10 \mathrm{~B}$ & $1.399(5)$ \\
\hline $\mathrm{C} 19 \mathrm{C}-\mathrm{H} 19 \mathrm{C}$ & 0.9300 & $\mathrm{C} 10 \mathrm{~B}-\mathrm{H} 10 \mathrm{~B}$ & $0.96(4)$ \\
\hline $\mathrm{C} 8 \mathrm{C}-\mathrm{C} 13 \mathrm{C}$ & $1.35(4)$ & $\mathrm{C} 10 \mathrm{~B}-\mathrm{C} 11 \mathrm{~B}$ & $1.372(6)$ \\
\hline $\mathrm{C} 8 \mathrm{C}-\mathrm{C} 9 \mathrm{C}$ & $1.37(4)$ & C11B-H11B & $1.04(5)$ \\
\hline $\mathrm{C} 13 \mathrm{C}-\mathrm{H} 13 \mathrm{C}$ & 0.9300 & $\mathrm{C} 11 \mathrm{~B}-\mathrm{C} 12 \mathrm{~B}$ & $1.367(6)$ \\
\hline $\mathrm{C} 13 \mathrm{C}-\mathrm{C} 12 \mathrm{C}$ & $1.39(4)$ & $\mathrm{C} 12 \mathrm{~B}-\mathrm{H} 12 \mathrm{~B}$ & $1.11(6)$ \\
\hline $\mathrm{C} 12 \mathrm{C}-\mathrm{H} 12 \mathrm{C}$ & 0.9300 & $\mathrm{C} 12 \mathrm{~B}-\mathrm{C} 13 \mathrm{~B}$ & $1.381(5)$ \\
\hline $\mathrm{C} 12 \mathrm{C}-\mathrm{C} 11 \mathrm{C}$ & $1.34(3)$ & $\mathrm{C} 13 \mathrm{~B}-\mathrm{H} 13 \mathrm{~B}$ & $0.97(3)$ \\
\hline $\mathrm{C} 11 \mathrm{C}-\mathrm{H} 11 \mathrm{C}$ & 0.9300 & $\mathrm{O} 1^{\prime}-\mathrm{H} 1^{\prime}$ & $0.99(5)$ \\
\hline $\mathrm{C} 11 \mathrm{C}-\mathrm{C} 10 \mathrm{C}$ & 1.402 (19) & $\mathrm{O} 1^{\prime}-\mathrm{C}^{\prime}$ & $1.308(3)$ \\
\hline $\mathrm{C} 10 \mathrm{C}-\mathrm{H} 10 \mathrm{C}$ & 0.9300 & $\mathrm{O} 2^{\prime}-\mathrm{C}^{\prime}$ & $1.202(3)$ \\
\hline $\mathrm{C} 10 \mathrm{C}-\mathrm{C} 9 \mathrm{C}$ & $1.29(4)$ & $\mathrm{C} 1^{\prime}-\mathrm{C} 2^{\prime}$ & $1.383(3)$ \\
\hline $\mathrm{C} 9 \mathrm{C}-\mathrm{H} 9 \mathrm{C}$ & 0.9300 & $\mathrm{C} 1^{\prime}-\mathrm{C}^{\prime}$ & $1.383(3)$ \\
\hline $\mathrm{O} 1 \mathrm{~A}-\mathrm{N} 3 \mathrm{~A}$ & $1.228(3)$ & $\mathrm{C} 1^{\prime}-\mathrm{C} 7^{\prime}$ & $1.492(3)$ \\
\hline $\mathrm{O} 2 \mathrm{~A}-\mathrm{N} 3 \mathrm{~A}$ & $1.228(3)$ & $\mathrm{C} 2^{\prime}-\mathrm{H} 2^{\prime}$ & $0.98(3)$ \\
\hline $\mathrm{O} 4 \mathrm{~A}-\mathrm{C} 7 \mathrm{~A}$ & $1.203(4)$ & $\mathrm{C} 2^{\prime}-\mathrm{C} 3^{\prime}$ & $1.388(3)$ \\
\hline $\mathrm{N} 2 \mathrm{~A}-\mathrm{C} 1 \mathrm{~A}$ & $1.331(3)$ & $\mathrm{C} 3^{\prime}-\mathrm{C} 4^{\prime}$ & $1.384(3)$ \\
\hline $\mathrm{N} 2 \mathrm{~A}-\mathrm{C} 2 \mathrm{~A}$ & $1.356(4)$ & $\mathrm{C} 4^{\prime}-\mathrm{H} 4^{\prime} \mathrm{A}$ & $0.95(3)$ \\
\hline $\mathrm{N} 3 \mathrm{~A}-\mathrm{C} 3 \mathrm{~A}$ & $1.419(4)$ & $\mathrm{C} 4^{\prime}-\mathrm{C} 5^{\prime}$ & $1.385(4)$ \\
\hline $\mathrm{C} 1 \mathrm{~A}-\mathrm{C} 4 \mathrm{~A}$ & $1.476(4)$ & $\mathrm{C} 5^{\prime}-\mathrm{C} 6^{\prime}$ & $1.391(3)$ \\
\hline $\mathrm{C} 2 \mathrm{~A}-\mathrm{H} 2 \mathrm{~A}$ & $0.93(3)$ & $\mathrm{C} 6^{\prime}-\mathrm{H} 6^{\prime}$ & $0.99(3)$ \\
\hline $\mathrm{C} 2 \mathrm{~A}-\mathrm{C} 3 \mathrm{~A}$ & $1.356(4)$ & & \\
\hline $\mathrm{C} 7 \mathrm{C}-\mathrm{O} 3 \mathrm{C}-\mathrm{C} 6 \mathrm{C}$ & $116.0(2)$ & $\mathrm{C} 6 \mathrm{~A}-\mathrm{C} 5 \mathrm{~A}-\mathrm{H} 5 \mathrm{AA}$ & $105.1(18)$ \\
\hline $\mathrm{C} 7 \mathrm{~A}-\mathrm{O} 3 \mathrm{~A}-\mathrm{C} 6 \mathrm{~A}$ & $113.8(2)$ & $\mathrm{C} 6 \mathrm{~A}-\mathrm{C} 5 \mathrm{~A}-\mathrm{H} 5 \mathrm{AB}$ & $110.2(18)$ \\
\hline $\mathrm{C} 1 \mathrm{~A}-\mathrm{N} 1 \mathrm{~A}-\mathrm{C} 3 \mathrm{~A}$ & $104.7(2)$ & $\mathrm{O} 3 \mathrm{~A}-\mathrm{C} 6 \mathrm{~A}-\mathrm{C} 5 \mathrm{~A}$ & $110.0(2)$ \\
\hline $\mathrm{C} 1 \mathrm{~A}-\mathrm{N} 1 \mathrm{~A}-\mathrm{C} 5 \mathrm{~A}$ & $124.2(2)$ & $\mathrm{O} 3 \mathrm{~A}-\mathrm{C} 6 \mathrm{~A}-\mathrm{H} 6 \mathrm{AA}$ & $108.9(17)$ \\
\hline $\mathrm{C} 3 \mathrm{~A}-\mathrm{N} 1 \mathrm{~A}-\mathrm{C} 5 \mathrm{~A}$ & $130.8(2)$ & $\mathrm{O} 3 \mathrm{~A}-\mathrm{C} 6 \mathrm{~A}-\mathrm{H} 6 \mathrm{AB}$ & $110(2)$ \\
\hline $\mathrm{C} 7 \mathrm{~B}-\mathrm{O} 3 \mathrm{~B}-\mathrm{C} 6 \mathrm{~B}$ & $115.5(2)$ & $\mathrm{C} 5 \mathrm{~A}-\mathrm{C} 6 \mathrm{~A}-\mathrm{H} 6 \mathrm{AA}$ & $106.5(18)$ \\
\hline $\mathrm{C} 3^{\prime}-\mathrm{O} 3^{\prime}-\mathrm{H} 3^{\prime}$ & $113(2)$ & $\mathrm{C} 5 \mathrm{~A}-\mathrm{C} 6 \mathrm{~A}-\mathrm{H} 6 \mathrm{AB}$ & $111.7(19)$ \\
\hline $\mathrm{C} 5^{\prime}-\mathrm{O} 4^{\prime}-\mathrm{H} 4^{\prime}$ & $112(2)$ & $\mathrm{H} 6 \mathrm{AA}-\mathrm{C} 6 \mathrm{~A}-\mathrm{H} 6 \mathrm{AB}$ & $109(3)$ \\
\hline $\mathrm{C} 1 \mathrm{C}-\mathrm{N} 1 \mathrm{C}-\mathrm{C} 3 \mathrm{C}$ & $105.6(2)$ & $\mathrm{O} 3 \mathrm{~A}-\mathrm{C} 7 \mathrm{~A}-\mathrm{C} 8 \mathrm{~A}$ & $114.4(3)$ \\
\hline $\mathrm{C} 1 \mathrm{C}-\mathrm{N} 1 \mathrm{C}-\mathrm{C} 5 \mathrm{C}$ & $124.8(3)$ & $\mathrm{O} 4 \mathrm{~A}-\mathrm{C} 7 \mathrm{~A}-\mathrm{O} 3 \mathrm{~A}$ & $122.6(3)$ \\
\hline $\mathrm{C} 3 \mathrm{C}-\mathrm{N} 1 \mathrm{C}-\mathrm{C} 5 \mathrm{C}$ & $129.3(2)$ & $\mathrm{O} 4 \mathrm{~A}-\mathrm{C} 7 \mathrm{~A}-\mathrm{C} 8 \mathrm{~A}$ & $123.0(3)$ \\
\hline $\mathrm{C} 1 \mathrm{C}-\mathrm{N} 2 \mathrm{C}-\mathrm{C} 2 \mathrm{C}$ & $107.1(2)$ & $\mathrm{C} 9 \mathrm{~A}-\mathrm{C} 8 \mathrm{~A}-\mathrm{C} 7 \mathrm{~A}$ & $123.2(3)$ \\
\hline $\mathrm{O} 1 \mathrm{C}-\mathrm{N} 3 \mathrm{C}-\mathrm{C} 3 \mathrm{C}$ & $116.7(3)$ & $\mathrm{C} 9 \mathrm{~A}-\mathrm{C} 8 \mathrm{~A}-\mathrm{C} 13 \mathrm{~A}$ & $119.2(3)$ \\
\hline $\mathrm{O} 2 \mathrm{C}-\mathrm{N} 3 \mathrm{C}-\mathrm{O} 1 \mathrm{C}$ & $123.8(3)$ & $\mathrm{C} 13 \mathrm{~A}-\mathrm{C} 8 \mathrm{~A}-\mathrm{C} 7 \mathrm{~A}$ & $117.6(3)$ \\
\hline $\mathrm{O} 2 \mathrm{C}-\mathrm{N} 3 \mathrm{C}-\mathrm{C} 3 \mathrm{C}$ & $119.5(2)$ & $\mathrm{C} 8 \mathrm{~A}-\mathrm{C} 9 \mathrm{~A}-\mathrm{H} 9 \mathrm{~A}$ & $120.2(19)$ \\
\hline $\mathrm{N} 1 \mathrm{C}-\mathrm{C} 1 \mathrm{C}-\mathrm{C} 4 \mathrm{C}$ & $124.1(3)$ & $\mathrm{C} 8 \mathrm{~A}-\mathrm{C} 9 \mathrm{~A}-\mathrm{C} 10 \mathrm{~A}$ & $119.9(4)$ \\
\hline $\mathrm{N} 2 \mathrm{C}-\mathrm{C} 1 \mathrm{C}-\mathrm{N} 1 \mathrm{C}$ & $111.1(3)$ & $\mathrm{C} 10 \mathrm{~A}-\mathrm{C} 9 \mathrm{~A}-\mathrm{H} 9 \mathrm{~A}$ & 119.9 (19) \\
\hline $\mathrm{N} 2 \mathrm{C}-\mathrm{C} 1 \mathrm{C}-\mathrm{C} 4 \mathrm{C}$ & $124.8(3)$ & $\mathrm{C} 9 \mathrm{~A}-\mathrm{C} 10 \mathrm{~A}-\mathrm{H} 10 \mathrm{~A}$ & $115(3)$ \\
\hline $\mathrm{N} 2 \mathrm{C}-\mathrm{C} 2 \mathrm{C}-\mathrm{H} 2 \mathrm{C}$ & $124(2)$ & $\mathrm{C} 11 \mathrm{~A}-\mathrm{C} 10 \mathrm{~A}-\mathrm{C} 9 \mathrm{~A}$ & $119.8(5)$ \\
\hline
\end{tabular}




\begin{tabular}{|c|c|}
\hline $\mathrm{N} 2 \mathrm{C}-\mathrm{C} 2 \mathrm{C}-\mathrm{C} 3 \mathrm{C}$ & $108.8(3)$ \\
\hline $\mathrm{C} 3 \mathrm{C}-\mathrm{C} 2 \mathrm{C}-\mathrm{H} 2 \mathrm{C}$ & $128(2)$ \\
\hline $\mathrm{N} 1 \mathrm{C}-\mathrm{C} 3 \mathrm{C}-\mathrm{N} 3 \mathrm{C}$ & $124.9(2)$ \\
\hline $\mathrm{C} 2 \mathrm{C}-\mathrm{C} 3 \mathrm{C}-\mathrm{N} 1 \mathrm{C}$ & $107.5(3)$ \\
\hline $\mathrm{C} 2 \mathrm{C}-\mathrm{C} 3 \mathrm{C}-\mathrm{N} 3 \mathrm{C}$ & $127.6(3)$ \\
\hline $\mathrm{C} 1 \mathrm{C}-\mathrm{C} 4 \mathrm{C}-\mathrm{H} 4 \mathrm{CA}$ & $117(3)$ \\
\hline $\mathrm{C} 1 \mathrm{C}-\mathrm{C} 4 \mathrm{C}-\mathrm{H} 4 \mathrm{CB}$ & $107(4)$ \\
\hline $\mathrm{C} 1 \mathrm{C}-\mathrm{C} 4 \mathrm{C}-\mathrm{H} 4 \mathrm{CC}$ & $113(4)$ \\
\hline $\mathrm{H} 4 \mathrm{CA}-\mathrm{C} 4 \mathrm{C}-\mathrm{H} 4 \mathrm{CB}$ & $110(5)$ \\
\hline $\mathrm{H} 4 \mathrm{CA}-\mathrm{C} 4 \mathrm{C}-\mathrm{H} 4 \mathrm{CC}$ & $104(5)$ \\
\hline $\mathrm{H} 4 \mathrm{CB}-\mathrm{C} 4 \mathrm{C}-\mathrm{H} 4 \mathrm{CC}$ & $105(5)$ \\
\hline $\mathrm{N} 1 \mathrm{C}-\mathrm{C} 5 \mathrm{C}-\mathrm{H} 5 \mathrm{CA}$ & $110.2(17)$ \\
\hline $\mathrm{N} 1 \mathrm{C}-\mathrm{C} 5 \mathrm{C}-\mathrm{H} 5 \mathrm{CB}$ & $107.7(17)$ \\
\hline $\mathrm{N} 1 \mathrm{C}-\mathrm{C} 5 \mathrm{C}-\mathrm{C} 6 \mathrm{C}$ & $114.2(3)$ \\
\hline $\mathrm{H} 5 \mathrm{CA}-\mathrm{C} 5 \mathrm{C}-\mathrm{H} 5 \mathrm{CB}$ & $106(2)$ \\
\hline $\mathrm{C} 6 \mathrm{C}-\mathrm{C} 5 \mathrm{C}-\mathrm{H} 5 \mathrm{CA}$ & $109.3(17)$ \\
\hline $\mathrm{C} 6 \mathrm{C}-\mathrm{C} 5 \mathrm{C}-\mathrm{H} 5 \mathrm{CB}$ & $108.8(17)$ \\
\hline $\mathrm{O} 3 \mathrm{C}-\mathrm{C} 6 \mathrm{C}-\mathrm{C} 5 \mathrm{C}$ & $108.4(2)$ \\
\hline $\mathrm{O} 3 \mathrm{C}-\mathrm{C} 6 \mathrm{C}-\mathrm{H} 6 \mathrm{CA}$ & $109.5(19)$ \\
\hline $\mathrm{O} 3 \mathrm{C}-\mathrm{C} 6 \mathrm{C}-\mathrm{H} 6 \mathrm{CB}$ & $108.9(17)$ \\
\hline $\mathrm{C} 5 \mathrm{C}-\mathrm{C} 6 \mathrm{C}-\mathrm{H} 6 \mathrm{CA}$ & $110.9(19)$ \\
\hline $\mathrm{C} 5 \mathrm{C}-\mathrm{C} 6 \mathrm{C}-\mathrm{H} 6 \mathrm{CB}$ & $108.6(17)$ \\
\hline $\mathrm{H} 6 \mathrm{CA}-\mathrm{C} 6 \mathrm{C}-\mathrm{H} 6 \mathrm{CB}$ & $110(2)$ \\
\hline $\mathrm{O} 3 \mathrm{C}-\mathrm{C} 7 \mathrm{C}-\mathrm{C} 14 \mathrm{C}$ & $105.9(12)$ \\
\hline $\mathrm{O} 3 \mathrm{C}-\mathrm{C} 7 \mathrm{C}-\mathrm{C} 8 \mathrm{C}$ & $120.0(13)$ \\
\hline $\mathrm{O} 4 \mathrm{C}-\mathrm{C} 7 \mathrm{C}-\mathrm{O} 3 \mathrm{C}$ & $122.2(3)$ \\
\hline $\mathrm{O} 4 \mathrm{C}-\mathrm{C} 7 \mathrm{C}-\mathrm{C} 14 \mathrm{C}$ & $131.9(12)$ \\
\hline $\mathrm{O} 4 \mathrm{C}-\mathrm{C} 7 \mathrm{C}-\mathrm{C} 8 \mathrm{C}$ & $117.9(13)$ \\
\hline $\mathrm{C} 15 \mathrm{C}-\mathrm{C} 14 \mathrm{C}-\mathrm{C} 7 \mathrm{C}$ & $127(2)$ \\
\hline $\mathrm{C} 15 \mathrm{C}-\mathrm{C} 14 \mathrm{C}-\mathrm{C} 19 \mathrm{C}$ & $115.6(14)$ \\
\hline $\mathrm{C} 19 \mathrm{C}-\mathrm{C} 14 \mathrm{C}-\mathrm{C} 7 \mathrm{C}$ & $118(2)$ \\
\hline $\mathrm{C} 14 \mathrm{C}-\mathrm{C} 15 \mathrm{C}-\mathrm{H} 15 \mathrm{C}$ & 119.2 \\
\hline $\mathrm{C} 14 \mathrm{C}-\mathrm{C} 15 \mathrm{C}-\mathrm{C} 16 \mathrm{C}$ & $121.7(15)$ \\
\hline $\mathrm{C} 16 \mathrm{C}-\mathrm{C} 15 \mathrm{C}-\mathrm{H} 15 \mathrm{C}$ & 119.2 \\
\hline $\mathrm{C} 15 \mathrm{C}-\mathrm{C} 16 \mathrm{C}-\mathrm{H} 16 \mathrm{C}$ & 118.7 \\
\hline $\mathrm{C} 17 \mathrm{C}-\mathrm{C} 16 \mathrm{C}-\mathrm{C} 15 \mathrm{C}$ & $122.5(13)$ \\
\hline $\mathrm{C} 17 \mathrm{C}-\mathrm{C} 16 \mathrm{C}-\mathrm{H} 16 \mathrm{C}$ & 118.7 \\
\hline $\mathrm{C} 16 \mathrm{C}-\mathrm{C} 17 \mathrm{C}-\mathrm{H} 17 \mathrm{C}$ & 122.0 \\
\hline $\mathrm{C} 16 \mathrm{C}-\mathrm{C} 17 \mathrm{C}-\mathrm{C} 18 \mathrm{C}$ & $115.9(14)$ \\
\hline $\mathrm{C} 18 \mathrm{C}-\mathrm{C} 17 \mathrm{C}-\mathrm{H} 17 \mathrm{C}$ & 122.0 \\
\hline $\mathrm{C} 17 \mathrm{C}-\mathrm{C} 18 \mathrm{C}-\mathrm{H} 18 \mathrm{C}$ & 119.8 \\
\hline $\mathrm{C} 19 \mathrm{C}-\mathrm{C} 18 \mathrm{C}-\mathrm{C} 17 \mathrm{C}$ & $120.4(15)$ \\
\hline $\mathrm{C} 19 \mathrm{C}-\mathrm{C} 18 \mathrm{C}-\mathrm{H} 18 \mathrm{C}$ & 119.8 \\
\hline $\mathrm{C} 14 \mathrm{C}-\mathrm{C} 19 \mathrm{C}-\mathrm{H} 19 \mathrm{C}$ & 118.2 \\
\hline $\mathrm{C} 18 \mathrm{C}-\mathrm{C} 19 \mathrm{C}-\mathrm{C} 14 \mathrm{C}$ & $123.6(15)$ \\
\hline $\mathrm{C} 18 \mathrm{C}-\mathrm{C} 19 \mathrm{C}-\mathrm{H} 19 \mathrm{C}$ & 118.2 \\
\hline $\mathrm{C} 13 \mathrm{C}-\mathrm{C} 8 \mathrm{C}-\mathrm{C} 7 \mathrm{C}$ & $118(3)$ \\
\hline $\mathrm{C} 9 \mathrm{C}-\mathrm{C} 8 \mathrm{C}-\mathrm{C} 7 \mathrm{C}$ & $119(2)$ \\
\hline
\end{tabular}

\begin{tabular}{|c|c|}
\hline $\mathrm{C} 11 \mathrm{~A}-\mathrm{C} 10 \mathrm{~A}-\mathrm{H} 10 \mathrm{~A}$ & $125(3)$ \\
\hline $\mathrm{C} 10 \mathrm{~A}-\mathrm{C} 11 \mathrm{~A}-\mathrm{H} 11 \mathrm{~A}$ & $122(3)$ \\
\hline $\mathrm{C} 10 \mathrm{~A}-\mathrm{C} 11 \mathrm{~A}-\mathrm{C} 12 \mathrm{~A}$ & $121.0(4)$ \\
\hline $\mathrm{C} 12 \mathrm{~A}-\mathrm{C} 11 \mathrm{~A}-\mathrm{H} 11 \mathrm{~A}$ & $117(3)$ \\
\hline $\mathrm{C} 11 \mathrm{~A}-\mathrm{C} 12 \mathrm{~A}-\mathrm{H} 12 \mathrm{~A}$ & $120(2)$ \\
\hline $\mathrm{C} 11 \mathrm{~A}-\mathrm{C} 12 \mathrm{~A}-\mathrm{C} 13 \mathrm{~A}$ & $119.7(5)$ \\
\hline $\mathrm{C} 13 \mathrm{~A}-\mathrm{C} 12 \mathrm{~A}-\mathrm{H} 12 \mathrm{~A}$ & $120(2)$ \\
\hline $\mathrm{C} 8 \mathrm{~A}-\mathrm{C} 13 \mathrm{~A}-\mathrm{H} 13 \mathrm{~A}$ & $113(3)$ \\
\hline $\mathrm{C} 12 \mathrm{~A}-\mathrm{C} 13 \mathrm{~A}-\mathrm{C} 8 \mathrm{~A}$ & $120.3(5)$ \\
\hline $\mathrm{C} 12 \mathrm{~A}-\mathrm{C} 13 \mathrm{~A}-\mathrm{H} 13 \mathrm{~A}$ & $126(3)$ \\
\hline $\mathrm{C} 1 \mathrm{~B}-\mathrm{N} 1 \mathrm{~B}-\mathrm{C} 3 \mathrm{~B}$ & $105.3(2)$ \\
\hline $\mathrm{C} 1 \mathrm{~B}-\mathrm{N} 1 \mathrm{~B}-\mathrm{C} 5 \mathrm{~B}$ & $125.1(3)$ \\
\hline $\mathrm{C} 3 \mathrm{~B}-\mathrm{N} 1 \mathrm{~B}-\mathrm{C} 5 \mathrm{~B}$ & $129.4(2)$ \\
\hline $\mathrm{C} 1 \mathrm{~B}-\mathrm{N} 2 \mathrm{~B}-\mathrm{C} 2 \mathrm{~B}$ & $106.5(2)$ \\
\hline $\mathrm{O} 1 \mathrm{~B}-\mathrm{N} 3 \mathrm{~B}-\mathrm{C} 3 \mathrm{~B}$ & $116.6(3)$ \\
\hline $\mathrm{O} 2 \mathrm{~B}-\mathrm{N} 3 \mathrm{~B}-\mathrm{O} 1 \mathrm{~B}$ & $123.7(3)$ \\
\hline $\mathrm{O} 2 \mathrm{~B}-\mathrm{N} 3 \mathrm{~B}-\mathrm{C} 3 \mathrm{~B}$ & $119.7(3)$ \\
\hline $\mathrm{N} 1 \mathrm{~B}-\mathrm{C} 1 \mathrm{~B}-\mathrm{C} 4 \mathrm{~B}$ & $125.4(3)$ \\
\hline $\mathrm{N} 2 \mathrm{~B}-\mathrm{C} 1 \mathrm{~B}-\mathrm{N} 1 \mathrm{~B}$ & $111.1(2)$ \\
\hline $\mathrm{N} 2 \mathrm{~B}-\mathrm{C} 1 \mathrm{~B}-\mathrm{C} 4 \mathrm{~B}$ & $123.6(3)$ \\
\hline $\mathrm{N} 2 \mathrm{~B}-\mathrm{C} 2 \mathrm{~B}-\mathrm{H} 2 \mathrm{~B}$ & $124.3(17)$ \\
\hline $\mathrm{C} 3 \mathrm{~B}-\mathrm{C} 2 \mathrm{~B}-\mathrm{N} 2 \mathrm{~B}$ & $108.8(3)$ \\
\hline $\mathrm{C} 3 \mathrm{~B}-\mathrm{C} 2 \mathrm{~B}-\mathrm{H} 2 \mathrm{~B}$ & $126.8(17)$ \\
\hline $\mathrm{N} 1 \mathrm{~B}-\mathrm{C} 3 \mathrm{~B}-\mathrm{N} 3 \mathrm{~B}$ & $124.4(2)$ \\
\hline $\mathrm{C} 2 \mathrm{~B}-\mathrm{C} 3 \mathrm{~B}-\mathrm{N} 1 \mathrm{~B}$ & $108.2(2)$ \\
\hline $\mathrm{C} 2 \mathrm{~B}-\mathrm{C} 3 \mathrm{~B}-\mathrm{N} 3 \mathrm{~B}$ & $127.3(3)$ \\
\hline $\mathrm{C} 1 \mathrm{~B}-\mathrm{C} 4 \mathrm{~B}-\mathrm{H} 4 \mathrm{BA}$ & 109.5 \\
\hline $\mathrm{C} 1 \mathrm{~B}-\mathrm{C} 4 \mathrm{~B}-\mathrm{H} 4 \mathrm{BB}$ & 109.5 \\
\hline $\mathrm{C} 1 \mathrm{~B}-\mathrm{C} 4 \mathrm{~B}-\mathrm{H} 4 \mathrm{BC}$ & 109.5 \\
\hline $\mathrm{H} 4 \mathrm{BA}-\mathrm{C} 4 \mathrm{~B}-\mathrm{H} 4 \mathrm{BB}$ & 109.5 \\
\hline $\mathrm{H} 4 \mathrm{BA}-\mathrm{C} 4 \mathrm{~B}-\mathrm{H} 4 \mathrm{BC}$ & 109.5 \\
\hline $\mathrm{H} 4 \mathrm{BB}-\mathrm{C} 4 \mathrm{~B}-\mathrm{H} 4 \mathrm{BC}$ & 109.5 \\
\hline $\mathrm{N} 1 \mathrm{~B}-\mathrm{C} 5 \mathrm{~B}-\mathrm{H} 5 \mathrm{BA}$ & $108(2)$ \\
\hline $\mathrm{N} 1 \mathrm{~B}-\mathrm{C} 5 \mathrm{~B}-\mathrm{H} 5 \mathrm{BB}$ & $105.4(18)$ \\
\hline $\mathrm{N} 1 \mathrm{~B}-\mathrm{C} 5 \mathrm{~B}-\mathrm{C} 6 \mathrm{~B}$ & $112.0(3)$ \\
\hline $\mathrm{H} 5 \mathrm{BA}-\mathrm{C} 5 \mathrm{~B}-\mathrm{H} 5 \mathrm{BB}$ & $111(3)$ \\
\hline $\mathrm{C} 6 \mathrm{~B}-\mathrm{C} 5 \mathrm{~B}-\mathrm{H} 5 \mathrm{BA}$ & $111(2)$ \\
\hline $\mathrm{C} 6 \mathrm{~B}-\mathrm{C} 5 \mathrm{~B}-\mathrm{H} 5 \mathrm{BB}$ & $109.9(18)$ \\
\hline $\mathrm{O} 3 \mathrm{~B}-\mathrm{C} 6 \mathrm{~B}-\mathrm{C} 5 \mathrm{~B}$ & $108.0(2)$ \\
\hline $\mathrm{O} 3 \mathrm{~B}-\mathrm{C} 6 \mathrm{~B}-\mathrm{H} 6 \mathrm{BA}$ & $110.0(17)$ \\
\hline $\mathrm{O} 3 \mathrm{~B}-\mathrm{C} 6 \mathrm{~B}-\mathrm{H} 6 \mathrm{BB}$ & $107.2(18)$ \\
\hline $\mathrm{C} 5 \mathrm{~B}-\mathrm{C} 6 \mathrm{~B}-\mathrm{H} 6 \mathrm{BA}$ & $111.2(17)$ \\
\hline $\mathrm{C} 5 \mathrm{~B}-\mathrm{C} 6 \mathrm{~B}-\mathrm{H} 6 \mathrm{BB}$ & $110.5(17)$ \\
\hline $\mathrm{H} 6 \mathrm{BA}-\mathrm{C} 6 \mathrm{~B}-\mathrm{H} 6 \mathrm{BB}$ & $110(2)$ \\
\hline $\mathrm{O} 3 \mathrm{~B}-\mathrm{C} 7 \mathrm{~B}-\mathrm{C} 8 \mathrm{~B}$ & $113.5(2)$ \\
\hline $\mathrm{O} 4 \mathrm{~B}-\mathrm{C} 7 \mathrm{~B}-\mathrm{O} 3 \mathrm{~B}$ & $121.7(3)$ \\
\hline $\mathrm{O} 4 \mathrm{~B}-\mathrm{C} 7 \mathrm{~B}-\mathrm{C} 8 \mathrm{~B}$ & $124.7(3)$ \\
\hline $\mathrm{C} 9 \mathrm{~B}-\mathrm{C} 8 \mathrm{~B}-\mathrm{C} 7 \mathrm{~B}$ & $123.1(3)$ \\
\hline
\end{tabular}




\begin{tabular}{|c|c|}
\hline $\mathrm{C} 9 \mathrm{C}-\mathrm{C} 8 \mathrm{C}-\mathrm{C} 13 \mathrm{C}$ & $123.6(17)$ \\
\hline $\mathrm{C} 8 \mathrm{C}-\mathrm{C} 13 \mathrm{C}-\mathrm{H} 13 \mathrm{C}$ & 121.2 \\
\hline $\mathrm{C} 12 \mathrm{C}-\mathrm{C} 13 \mathrm{C}-\mathrm{C} 8 \mathrm{C}$ & 117.7 (19) \\
\hline $\mathrm{C} 12 \mathrm{C}-\mathrm{C} 13 \mathrm{C}-\mathrm{H} 13 \mathrm{C}$ & 121.2 \\
\hline $\mathrm{C} 13 \mathrm{C}-\mathrm{C} 12 \mathrm{C}-\mathrm{H} 12 \mathrm{C}$ & 120.3 \\
\hline $\mathrm{C} 11 \mathrm{C}-\mathrm{C} 12 \mathrm{C}-\mathrm{C} 13 \mathrm{C}$ & $119.5(17)$ \\
\hline $\mathrm{C} 11 \mathrm{C}-\mathrm{C} 12 \mathrm{C}-\mathrm{H} 12 \mathrm{C}$ & 120.3 \\
\hline $\mathrm{C} 12 \mathrm{C}-\mathrm{C} 11 \mathrm{C}-\mathrm{H} 11 \mathrm{C}$ & 120.3 \\
\hline $\mathrm{C} 12 \mathrm{C}-\mathrm{C} 11 \mathrm{C}-\mathrm{C} 10 \mathrm{C}$ & $119(2)$ \\
\hline $\mathrm{C} 10 \mathrm{C}-\mathrm{C} 11 \mathrm{C}-\mathrm{H} 11 \mathrm{C}$ & 120.3 \\
\hline $\mathrm{C} 11 \mathrm{C}-\mathrm{C} 10 \mathrm{C}-\mathrm{H} 10 \mathrm{C}$ & 118.8 \\
\hline $\mathrm{C} 9 \mathrm{C}-\mathrm{C} 10 \mathrm{C}-\mathrm{C} 11 \mathrm{C}$ & $122(2)$ \\
\hline $\mathrm{C} 9 \mathrm{C}-\mathrm{C} 10 \mathrm{C}-\mathrm{H} 10 \mathrm{C}$ & 118.8 \\
\hline $\mathrm{C} 8 \mathrm{C}-\mathrm{C} 9 \mathrm{C}-\mathrm{H} 9 \mathrm{C}$ & 121.3 \\
\hline $\mathrm{C} 10 \mathrm{C}-\mathrm{C} 9 \mathrm{C}-\mathrm{C} 8 \mathrm{C}$ & $117.4(16)$ \\
\hline $\mathrm{C} 10 \mathrm{C}-\mathrm{C} 9 \mathrm{C}-\mathrm{H} 9 \mathrm{C}$ & 121.3 \\
\hline $\mathrm{C} 1 \mathrm{~A}-\mathrm{N} 2 \mathrm{~A}-\mathrm{C} 2 \mathrm{~A}$ & $105.9(2)$ \\
\hline $\mathrm{O} 1 \mathrm{~A}-\mathrm{N} 3 \mathrm{~A}-\mathrm{C} 3 \mathrm{~A}$ & $117.2(3)$ \\
\hline $\mathrm{O} 2 \mathrm{~A}-\mathrm{N} 3 \mathrm{~A}-\mathrm{O} 1 \mathrm{~A}$ & $123.5(3)$ \\
\hline $\mathrm{O} 2 \mathrm{~A}-\mathrm{N} 3 \mathrm{~A}-\mathrm{C} 3 \mathrm{~A}$ & $119.2(3)$ \\
\hline $\mathrm{N} 1 \mathrm{~A}-\mathrm{C} 1 \mathrm{~A}-\mathrm{C} 4 \mathrm{~A}$ & $124.5(2)$ \\
\hline $\mathrm{N} 2 \mathrm{~A}-\mathrm{C} 1 \mathrm{~A}-\mathrm{N} 1 \mathrm{~A}$ & $112.1(2)$ \\
\hline $\mathrm{N} 2 \mathrm{~A}-\mathrm{C} 1 \mathrm{~A}-\mathrm{C} 4 \mathrm{~A}$ & $123.5(2)$ \\
\hline $\mathrm{N} 2 \mathrm{~A}-\mathrm{C} 2 \mathrm{~A}-\mathrm{H} 2 \mathrm{~A}$ & $124.5(19)$ \\
\hline $\mathrm{N} 2 \mathrm{~A}-\mathrm{C} 2 \mathrm{~A}-\mathrm{C} 3 \mathrm{~A}$ & $109.2(3)$ \\
\hline $\mathrm{C} 3 \mathrm{~A}-\mathrm{C} 2 \mathrm{~A}-\mathrm{H} 2 \mathrm{~A}$ & $126.3(19)$ \\
\hline $\mathrm{N} 1 \mathrm{~A}-\mathrm{C} 3 \mathrm{~A}-\mathrm{N} 3 \mathrm{~A}$ & $125.6(2)$ \\
\hline $\mathrm{C} 2 \mathrm{~A}-\mathrm{C} 3 \mathrm{~A}-\mathrm{N} 1 \mathrm{~A}$ & $108.0(2)$ \\
\hline $\mathrm{C} 2 \mathrm{~A}-\mathrm{C} 3 \mathrm{~A}-\mathrm{N} 3 \mathrm{~A}$ & $126.4(3)$ \\
\hline $\mathrm{C} 1 \mathrm{~A}-\mathrm{C} 4 \mathrm{~A}-\mathrm{H} 4 \mathrm{AA}$ & $113(2)$ \\
\hline $\mathrm{C} 1 \mathrm{~A}-\mathrm{C} 4 \mathrm{~A}-\mathrm{H} 4 \mathrm{AB}$ & $110(2)$ \\
\hline $\mathrm{C} 1 \mathrm{~A}-\mathrm{C} 4 \mathrm{~A}-\mathrm{H} 4 \mathrm{AC}$ & $112(3)$ \\
\hline $\mathrm{H} 4 \mathrm{AA}-\mathrm{C} 4 \mathrm{~A}-\mathrm{H} 4 \mathrm{AB}$ & $104(3)$ \\
\hline $\mathrm{H} 4 \mathrm{AA}-\mathrm{C} 4 \mathrm{~A}-\mathrm{H} 4 \mathrm{AC}$ & $112(3)$ \\
\hline $\mathrm{H} 4 \mathrm{AB}-\mathrm{C} 4 \mathrm{~A}-\mathrm{H} 4 \mathrm{AC}$ & $104(3)$ \\
\hline $\mathrm{N} 1 \mathrm{~A}-\mathrm{C} 5 \mathrm{~A}-\mathrm{H} 5 \mathrm{AA}$ & $107.6(18)$ \\
\hline $\mathrm{N} 1 \mathrm{~A}-\mathrm{C} 5 \mathrm{~A}-\mathrm{H} 5 \mathrm{AB}$ & $105.7(18)$ \\
\hline $\mathrm{N} 1 \mathrm{~A}-\mathrm{C} 5 \mathrm{~A}-\mathrm{C} 6 \mathrm{~A}$ & $115.0(3)$ \\
\hline $\mathrm{H} 5 \mathrm{AA}-\mathrm{C} 5 \mathrm{~A}-\mathrm{H} 5 \mathrm{AB}$ & $114(3)$ \\
\hline
\end{tabular}

\begin{tabular}{|c|c|}
\hline $\mathrm{C} 9 \mathrm{~B}-\mathrm{C} 8 \mathrm{~B}-\mathrm{C} 13 \mathrm{~B}$ & $119.3(3)$ \\
\hline $\mathrm{C} 13 \mathrm{~B}-\mathrm{C} 8 \mathrm{~B}-\mathrm{C} 7 \mathrm{~B}$ & $117.6(3)$ \\
\hline $\mathrm{C} 8 \mathrm{~B}-\mathrm{C} 9 \mathrm{~B}-\mathrm{H} 9 \mathrm{~B}$ & $117.2(19)$ \\
\hline $\mathrm{C} 8 \mathrm{~B}-\mathrm{C} 9 \mathrm{~B}-\mathrm{C} 10 \mathrm{~B}$ & $119.1(4)$ \\
\hline $\mathrm{C} 10 \mathrm{~B}-\mathrm{C} 9 \mathrm{~B}-\mathrm{H} 9 \mathrm{~B}$ & $123.6(19)$ \\
\hline $\mathrm{C} 9 \mathrm{~B}-\mathrm{C} 10 \mathrm{~B}-\mathrm{H} 10 \mathrm{~B}$ & $112(2)$ \\
\hline $\mathrm{C} 11 \mathrm{~B}-\mathrm{C} 10 \mathrm{~B}-\mathrm{C} 9 \mathrm{~B}$ & $120.0(4)$ \\
\hline $\mathrm{C} 11 \mathrm{~B}-\mathrm{C} 10 \mathrm{~B}-\mathrm{H} 10 \mathrm{~B}$ & $127(2)$ \\
\hline $\mathrm{C} 10 \mathrm{~B}-\mathrm{C} 11 \mathrm{~B}-\mathrm{H} 11 \mathrm{~B}$ & $122(3)$ \\
\hline $\mathrm{C} 12 \mathrm{~B}-\mathrm{C} 11 \mathrm{~B}-\mathrm{C} 10 \mathrm{~B}$ & $121.5(4)$ \\
\hline $\mathrm{C} 12 \mathrm{~B}-\mathrm{C} 11 \mathrm{~B}-\mathrm{H} 11 \mathrm{~B}$ & $117(3)$ \\
\hline $\mathrm{C} 11 \mathrm{~B}-\mathrm{C} 12 \mathrm{~B}-\mathrm{H} 12 \mathrm{~B}$ & $124(3)$ \\
\hline $\mathrm{C} 11 \mathrm{~B}-\mathrm{C} 12 \mathrm{~B}-\mathrm{C} 13 \mathrm{~B}$ & $118.2(4)$ \\
\hline $\mathrm{C} 13 \mathrm{~B}-\mathrm{C} 12 \mathrm{~B}-\mathrm{H} 12 \mathrm{~B}$ & $118(3)$ \\
\hline $\mathrm{C} 8 \mathrm{~B}-\mathrm{C} 13 \mathrm{~B}-\mathrm{H} 13 \mathrm{~B}$ & $119(2)$ \\
\hline $\mathrm{C} 12 \mathrm{~B}-\mathrm{C} 13 \mathrm{~B}-\mathrm{C} 8 \mathrm{~B}$ & $121.8(4)$ \\
\hline $\mathrm{C} 12 \mathrm{~B}-\mathrm{C} 13 \mathrm{~B}-\mathrm{H} 13 \mathrm{~B}$ & $119(2)$ \\
\hline $\mathrm{C} 7^{\prime}-\mathrm{O} 1^{\prime}-\mathrm{H} 1^{\prime}$ & $109(2)$ \\
\hline $\mathrm{C} 2^{\prime}-\mathrm{C} 1^{\prime}-\mathrm{C} 6^{\prime}$ & $121.5(2)$ \\
\hline $\mathrm{C} 2^{\prime}-\mathrm{C} 1^{\prime}-\mathrm{C} 7^{\prime}$ & $117.9(2)$ \\
\hline $\mathrm{C} 6^{\prime}-\mathrm{C} 1^{\prime}-\mathrm{C} 7^{\prime}$ & $120.6(2)$ \\
\hline $\mathrm{C} 1^{\prime}-\mathrm{C} 2^{\prime}-\mathrm{H} 2^{\prime}$ & $123.3(16)$ \\
\hline $\mathrm{C} 1^{\prime}-\mathrm{C} 2^{\prime}-\mathrm{C} 3^{\prime}$ & $118.9(2)$ \\
\hline $\mathrm{C} 3^{\prime}-\mathrm{C} 2^{\prime}-\mathrm{H} 2^{\prime}$ & $117.7(16)$ \\
\hline $\mathrm{O} 3^{\prime}-\mathrm{C} 3^{\prime}-\mathrm{C} 2^{\prime}$ & $117.5(2)$ \\
\hline $\mathrm{O} 3^{\prime}-\mathrm{C} 3^{\prime}-\mathrm{C} 4^{\prime}$ & $122.0(2)$ \\
\hline $\mathrm{C}^{\prime}-\mathrm{C} 3^{\prime}-\mathrm{C} 2^{\prime}$ & $120.5(2)$ \\
\hline $\mathrm{C}^{\prime}-\mathrm{C} 4^{\prime}-\mathrm{H} 4^{\prime} \mathrm{A}$ & $119.7(17)$ \\
\hline $\mathrm{C} 3^{\prime}-\mathrm{C} 4^{\prime}-\mathrm{C} 5^{\prime}$ & $119.9(2)$ \\
\hline $\mathrm{C}^{\prime}-\mathrm{C} 4^{\prime}-\mathrm{H} 4^{\prime} \mathrm{A}$ & $120.4(17)$ \\
\hline $\mathrm{O} 4^{\prime}-\mathrm{C} 5^{\prime}-\mathrm{C} 4^{\prime}$ & $122.1(2)$ \\
\hline $\mathrm{O} 4^{\prime}-\mathrm{C} 5^{\prime}-\mathrm{C} 6^{\prime}$ & $117.6(2)$ \\
\hline $\mathrm{C} 4^{\prime}-\mathrm{C} 5^{\prime}-\mathrm{C} 6^{\prime}$ & $120.3(2)$ \\
\hline $\mathrm{C} 1^{\prime}-\mathrm{C} 6^{\prime}-\mathrm{C} 5^{\prime}$ & $118.9(2)$ \\
\hline $\mathrm{C} 1^{\prime}-\mathrm{C} 6^{\prime}-\mathrm{H} 6^{\prime}$ & $121.7(15)$ \\
\hline $\mathrm{C} 5^{\prime}-\mathrm{C} 6^{\prime}-\mathrm{H} 6^{\prime}$ & $119.3(15)$ \\
\hline $\mathrm{O} 1^{\prime}-\mathrm{C}^{\prime}-\mathrm{C} 1^{\prime}$ & $113.8(2)$ \\
\hline $\mathrm{O} 2^{\prime}-\mathrm{C}^{\prime}-\mathrm{O} 1^{\prime}$ & $122.2(2)$ \\
\hline $\mathrm{O} 2^{\prime}-\mathrm{C} 7^{\prime}-\mathrm{C} 1^{\prime}$ & $124.0(2)$ \\
\hline
\end{tabular}

